Florida International University FIU Digital Commons

$12-8-2004$

\title{
Finite difference time domain analysis of fractal antennas used in wireless communications
}

Chritz Adenauer Duncan

Florida International University

DOI: $10.25148 /$ etd.FI15101247

Follow this and additional works at: https://digitalcommons.fiu.edu/etd

Part of the Electrical and Computer Engineering Commons

\section{Recommended Citation}

Duncan, Chritz Adenauer, "Finite difference time domain analysis of fractal antennas used in wireless communications" (2004). FIU Electronic Theses and Dissertations. 3100.

https://digitalcommons.fiu.edu/etd/3100 
FLORIDA INTERNATIONAL UNIVERSITY

Miami, Florida

FINITE DIFFERENCE TIME DOMAIN ANALYSIS

OF FRACTAL ANTENNAS USED IN

WIRELESS COMMUNICATIONS

A thesis submitted in partial fulfillment of the

requirements for the degree of

MASTER OF SCIENCE

in

ELECTRICAL ENGINEERING

by

Chritz Adenauer Duncan 
To: Dean Vish Prasad

College of Engineering

This thesis, written by Chritz Adenauer Duncan, and entitled Finite Difference Time Domain Analysis of Fractal Antennas Used in Wireless Communications, having been approved in respect to style and intellectual content, is referred to you for judgment.

We have read this thesis and recommend that it be approved.

Dr. Malek Adjouadi

Dr.Malcolm M. Heimer

Dr. Tadeusz M. Babij, Major Professor

Date of Defense: December 8, 2004

The thesis of Chritz Adenauer Duncan is approved.

Dean Vish Prasad College of Engineering

Dean Douglas Wartzok University Graduate School

Florida International University, 2004 


\section{DEDICATION}

I dedicate this thesis to my parents and to my brothers. Without their patience, support, sacrifice, understanding and love, the completion of this thesis work would not have been possible. 


\section{ACKNOWLEDGMENTS}

I would like to thank my major professor, Dr. Tadeusz M. Babij for his support and guidance throughout this work. I am grateful for his encouraging comments, suggestions and patience.

I would also like to thank Dr. Heimer for serving on my master thesis committee. I appreciate his encouraging comments.

A very special thanks must go to Dr. Adjouadi, for accepting to serve on my masters thesis committee. I am very grateful for his comments and suggestions.

Also, I would like to thank Edward Lule for his assistance, help and patience throughout this work. 


\section{ABSTRACT OF THE THESIS \\ FINITE DIFFERENCE TIME DOMAIN ANALYSIS OF FRACTAL ANTENNAS USED IN WIRELESS COMMUNICATIONS}

by

Chritz Adenauer Duncan

Florida International University, 2004

Miami, Florida

Professor Tadeusz M. Babij, Major Professor

The advances in wireless technology and the ever-growing demand for multiband and smaller antennas in wireless communications has led to the field of mathematics known as fractal. The use of fractal geometry in antenna design has created a significant amount of interest within the wireless communications societies and most importantly, antenna design.

This thesis investigates the performance and optimization of fractal antennas used in wireless communications. The principle analytical tool utilized in the study is the Finite Difference Time Domain technique (FDTD). This numerical method was applied to calculate the electromagnetic propagation characteristics of the Sierpinski gasket and Koch snowflake fractal antennas.

Numerical results were computed for the two fractal antennas and compared to a conventional antenna. The input impedance, radiation pattern, the return loss and far field condition of these antennas are computed and analyzed. The Finite Difference Time Domain (FDTD) simulated results were collected and showed to be in good agreement. 


\section{TABLE OF CONTENTS}

CHAPTER

PAGE

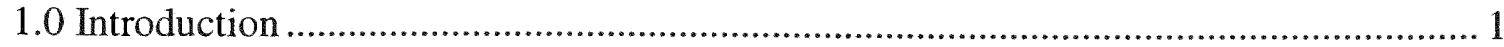

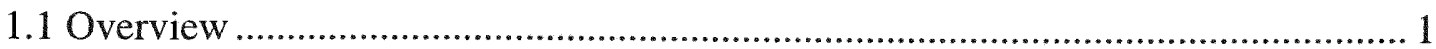

2.0 The Finite Difference Time Domain Method ............................................................ 4

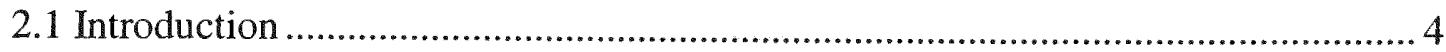

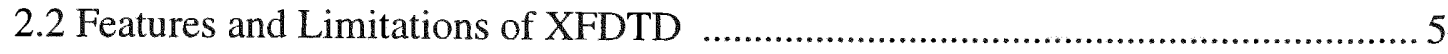

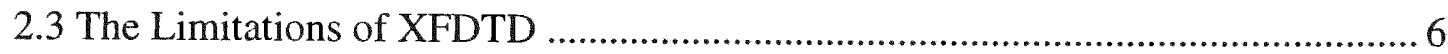

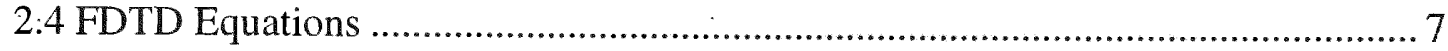

2.5 Maxwell's Equation Faraday's Law .................................................................. 7

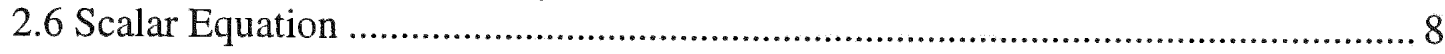

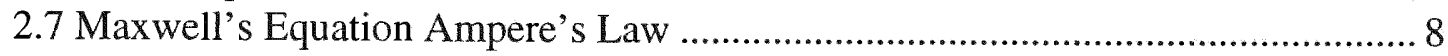

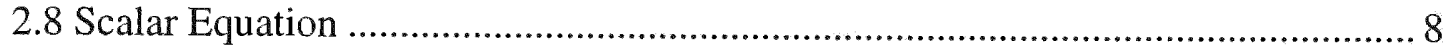

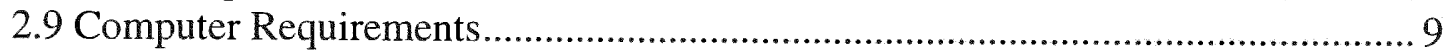

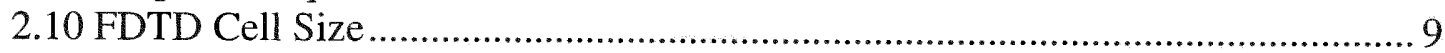

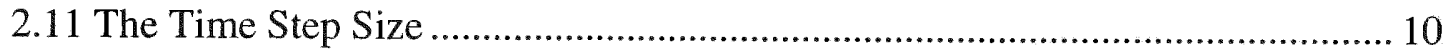

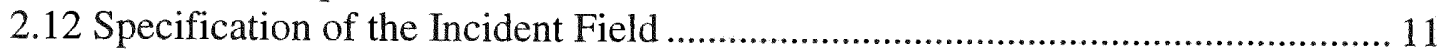

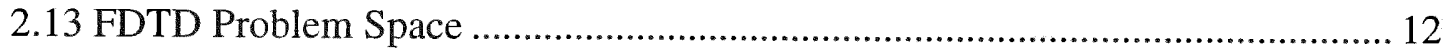

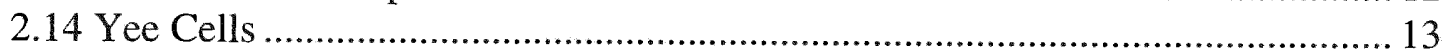

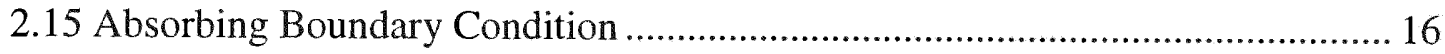

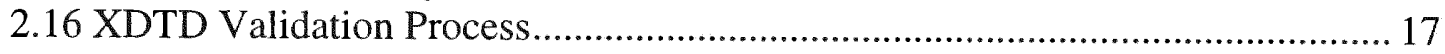

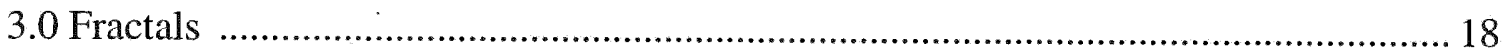

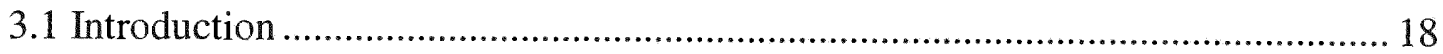

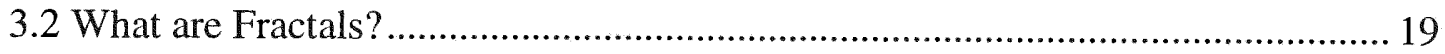

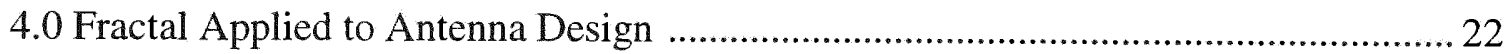

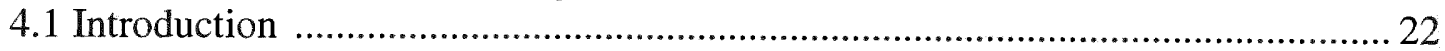

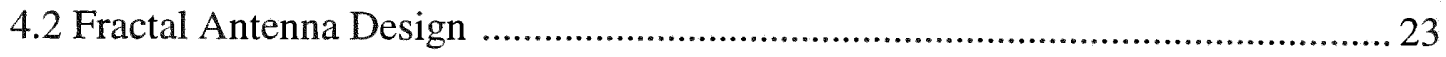

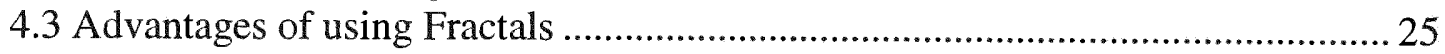

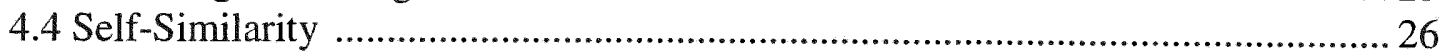

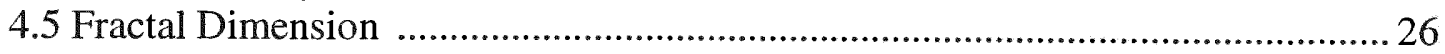

4.6 Fractal Antenna Configuration / Method .......................................................... 27

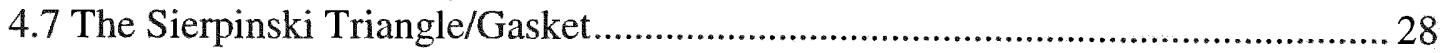

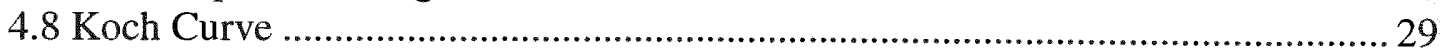

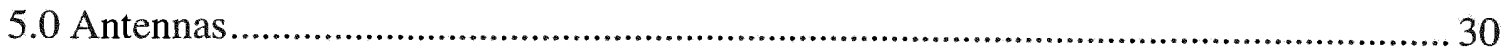

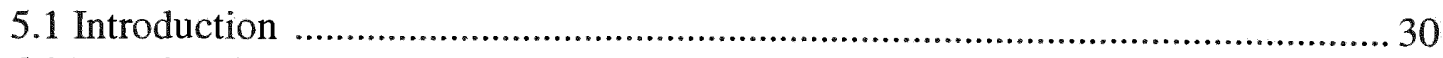

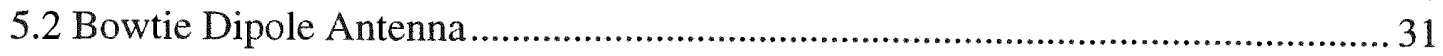

5.3 XFDTD Analysis of Bowtie Dipole Antenna ................................................... 32

5.4 Geometry of Bowtie Dipole Antenna................................................................. 33

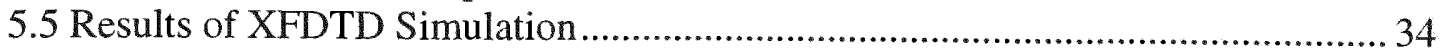


6.0 FDTD Analysis of Koch Snowflake Fractal Antenna............................................... 46

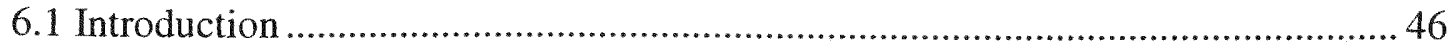

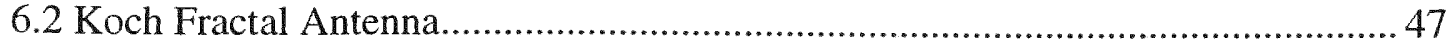

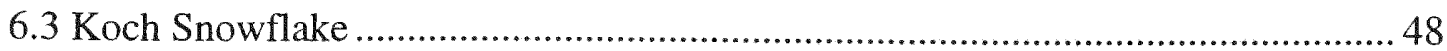

6.4 Geometry of Koch Snowflake Antenna ................................................................. 49

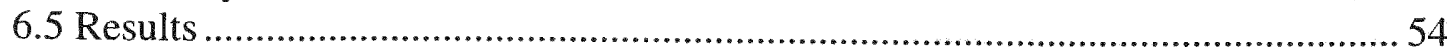

7.0 FDTD Analysis of Sierpinski Gasket Fractal Antenna _..........................................95

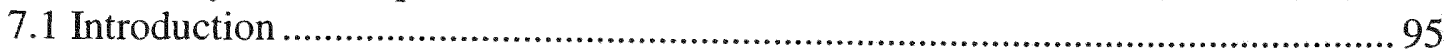

7.2 Geometry of Sierpinski Gasket Fractal ............................................................. 96

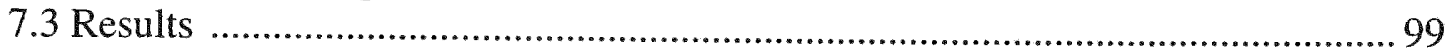

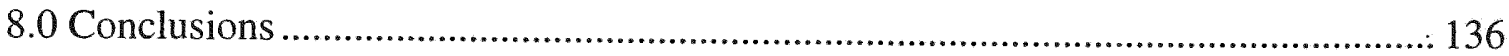

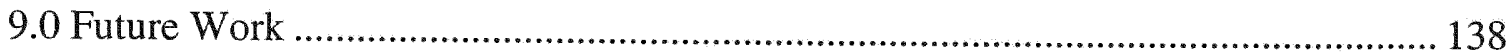

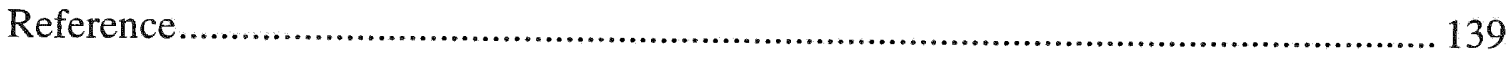


FIGURE

PAGE

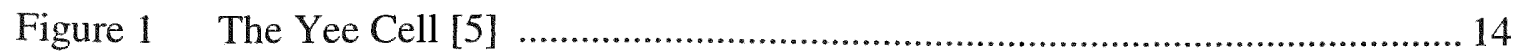

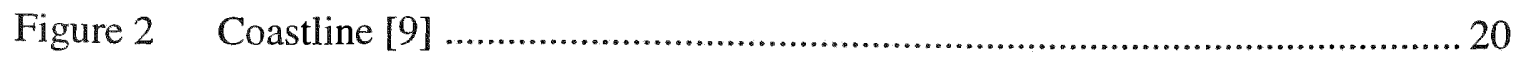

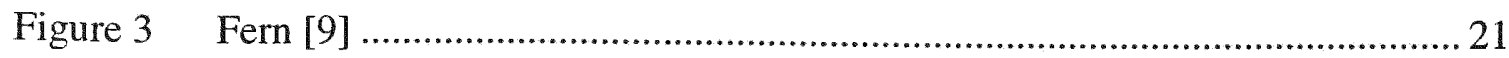

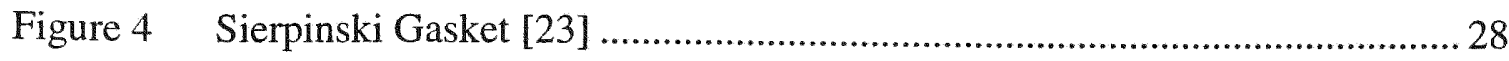

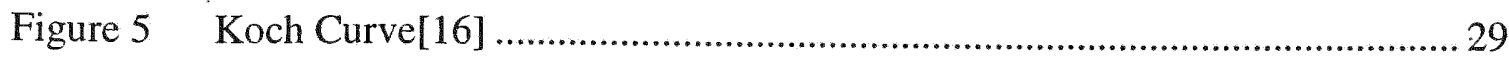

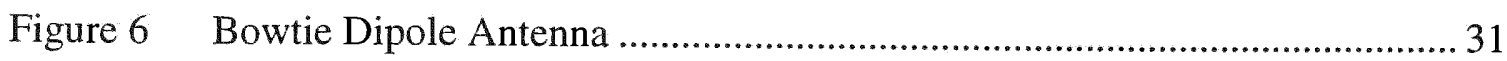

Figure 7 Geometry of Bowtie Dipole Antenna ...................................................... 33

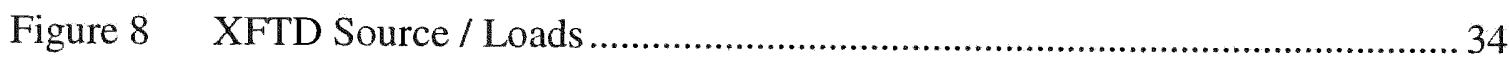

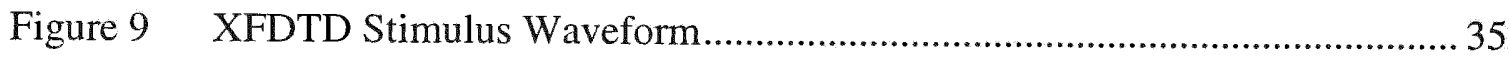

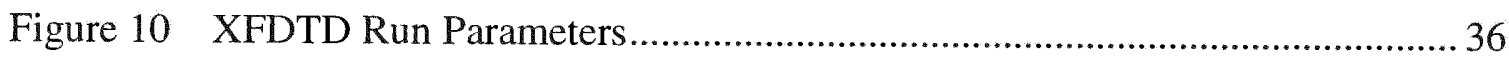

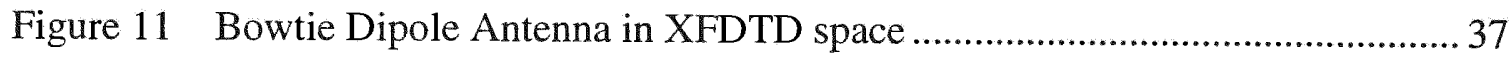

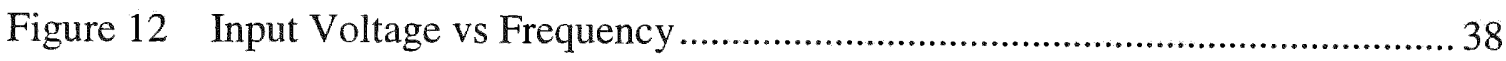

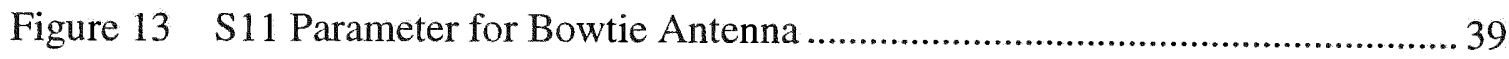

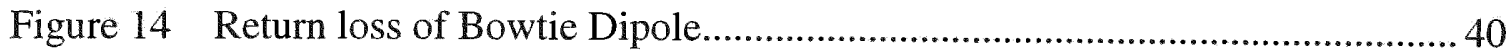

Figure 15 Input Impedance for Bowtie Dipole ...................................................... 41

Figure 16 Real and Imaginary Input Impedance for Bowtie Dipole............................ 42

Figure 17 FDTD simulated Gain of Bowtie Antenna .............................................. 43

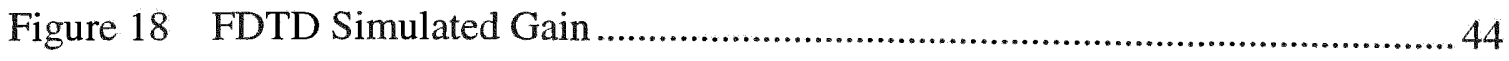

Figure 19 Simulated Radiation of Bowtie Dipole at resonant frequency .................... 45

Figure 20 Simulated Radiation of Bowtie Dipole at resonant frequency ...................... 45

Figure 21 Construction of Koch Curve[9] …..................................................... 47 
Figure 22 Outline of Various Iteration Stages of Koch Snowflake Fractal [9].............. 53

Figure 23 Geometry of Koch Snowflake Fractal Dipole Antenna [9] ..........................53

Figure 24 Source / Loads Parameters for Koch 0 ...................................................... 55

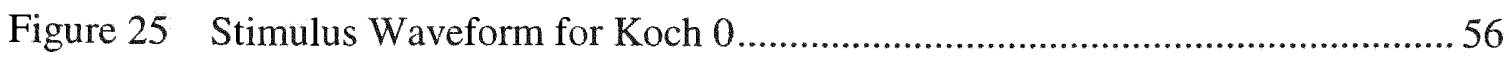

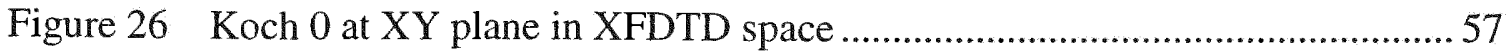

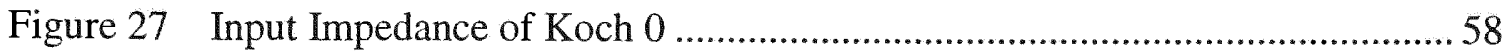

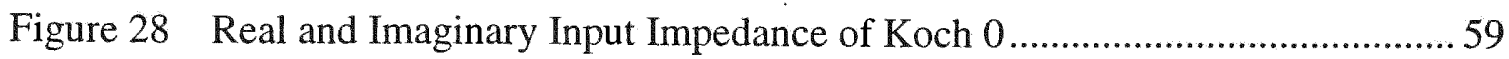

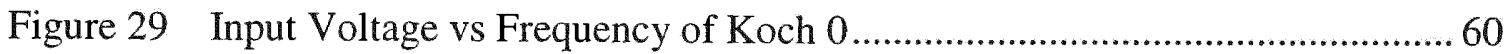

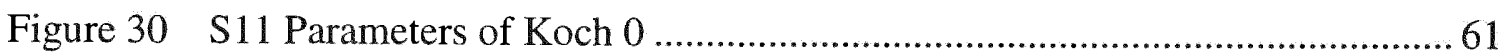

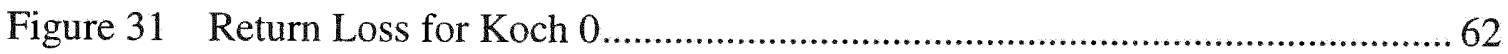

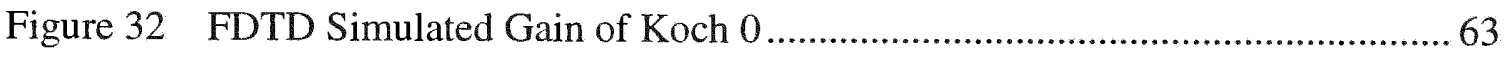

Figure 33 FDTD Simulated Constant Phi Gain of Koch 0 .......................................... 64

Figure 34 Simulated Radiation of Koch 0 at resonant ................................................6 65

Figure 35 Simulated Radiation of Koch 0 at resonant frequency ................................6 65

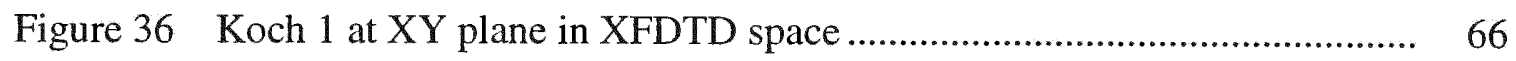

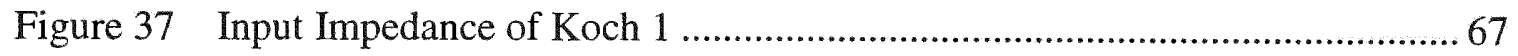

Figure 38 Real and Imaginary Input Impedance of Koch 1 ......................................6 68

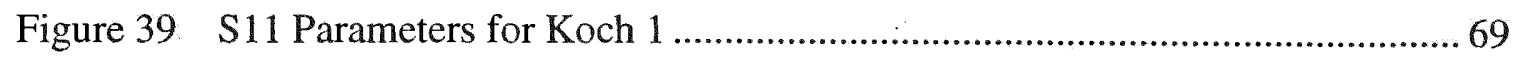

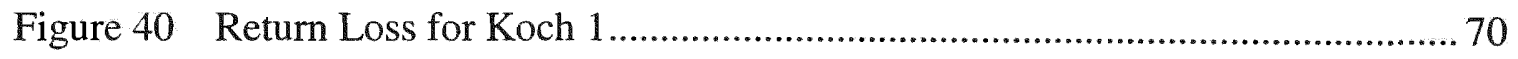

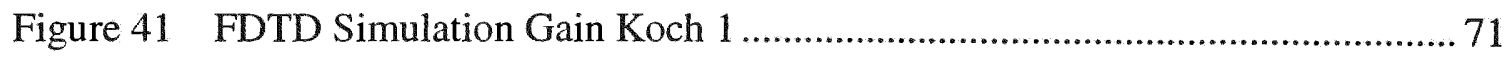

Figure 42 FDTD Simulation of Constant Phi Gain for Koch 1 ................................... 72

Figure 43 Simulated Radiation for Koch 1 at resonant frequency ............................ 73

Figure 44 Simulated Radiation for Koch 1 at resonant frequency .............................. 73 


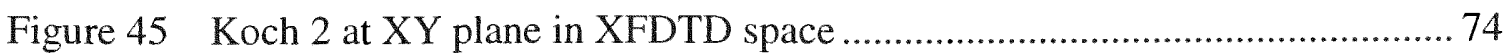

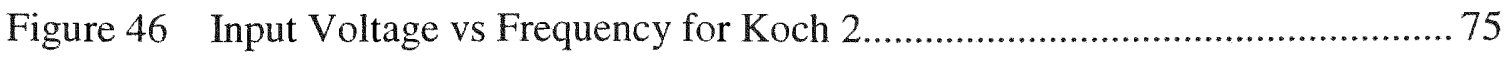

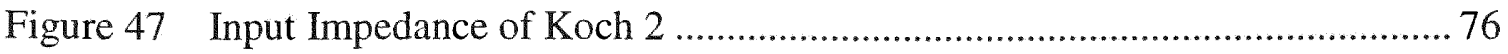

Figure $48 \quad$ Real and Imaginary Input Impedance of Koch 2 _..................................... 77

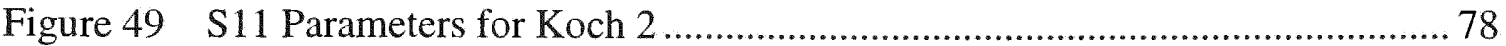

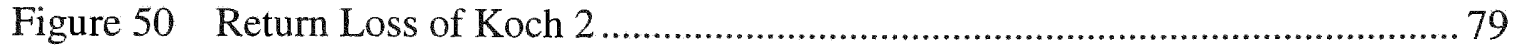

Figure 51 Simulated Radiation for Koch 2 at resonant frequency ............................... 80

Figure 52 Simulated Radiation for Koch 2 at resonant frequency ............................... 80

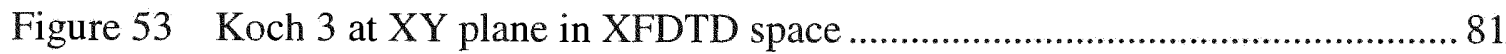

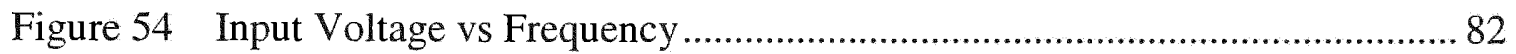

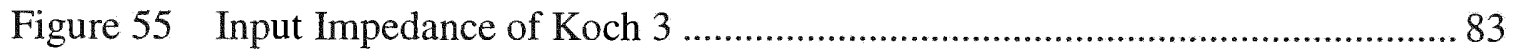

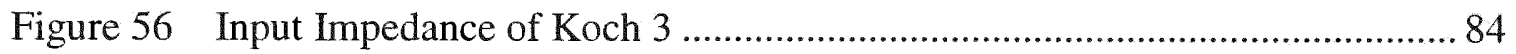

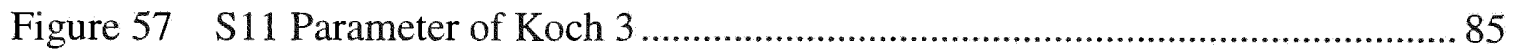

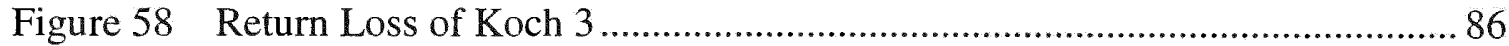

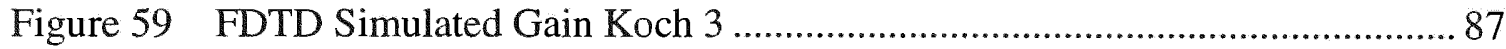

Figure 60 FDTD Simulated Constant Phi Gain Koch 3 ….......................................... 88

Figure 61 Simulated Radiation for Koch at resonant frequency ................................89

Figure 62 Simulated Radiation for Koch at resonant frequency .................................. 89

Figure 63 Return Loss of Koch Antenna with Various Iterations ...............................91

Figure 64 Input Resistance for Koch Fractal with different Iterations ..........................92

Figure 65 Return Loss for Bowtie and Koch 3 ....................................................... 93

Figure 66 Iteration of a Sierpinski Gasket Fractal Antenna [24] ................................96

Figure 67 Geometry of Sierpinski Gasket Dipole Antenna [24]................................97 
Figure 68 Geometry of Sierpinski Fractal

Figure 69 Run Parameter for Siepinski Gasket 0 .................................................... 100

Figure 70 Sierpinski 0 at XY Plane in XFDTD Space............................................... 101

Figure 71 Input Impedance of Sierpinski 0 ........................................................ 102

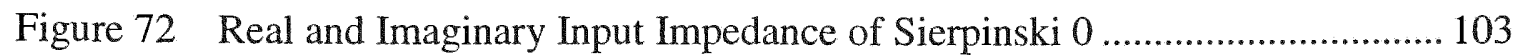

Figure 73 S11 Parameters of Sierpinski 0 .......................................................... 104

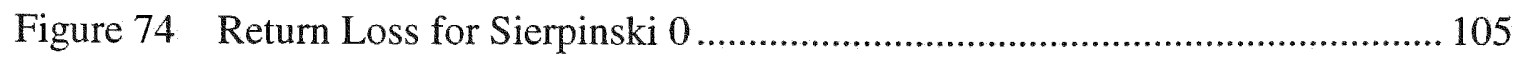

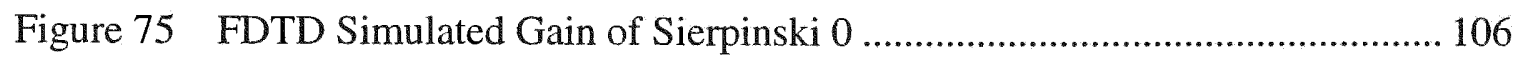

Figure 76 FDTD Simulated Constant Phi Gain of Sierpinski 0 ............................... 107

Figure 77 FDTD Run Parameter for Sierpinski 1 ................................................... 108

Figure 78 Sierpinski 1 at XY Plane in XFDTD Space.............................................. 109

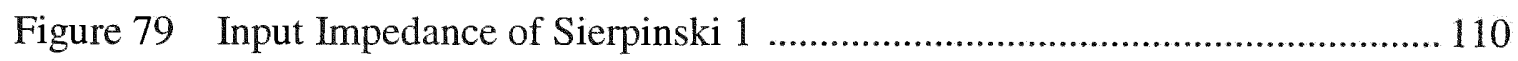

Figure 80 Real and Imaginary Input Impedance of Sierpinski 1 .............................. 111

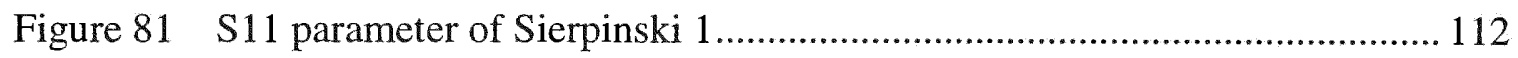

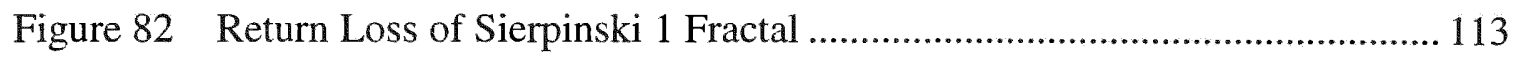

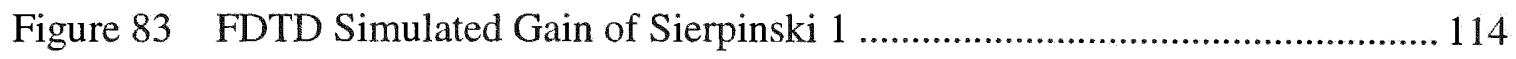

Figure 84 FDTD Simulated Constant Phi Gain of Sierpinski 1............................... 115

Figure 85 FDTD Run parameters for Sierpinski 2 ............................................... 116

Figure 86 Sierpinski 2 at XY Plane in XFDTD Space............................................. 117

Figure 87 Real and Imaginary Input Impedance of Sierpinski $2 \ldots \ldots \ldots \ldots \ldots \ldots \ldots \ldots \ldots \ldots \ldots . . . . . . . . . .118$

Figure 88 Real and Imaginary Input Impedance of Sierpinski $2 \ldots \ldots \ldots \ldots \ldots \ldots \ldots \ldots \ldots \ldots . . . \ldots 119$

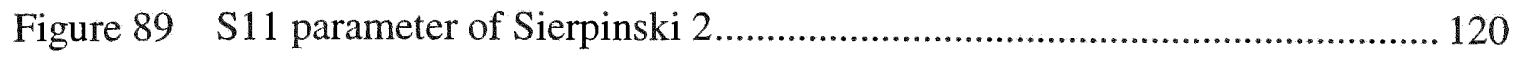

Figure 90 Return Loss of Sierpinski 2 Fractal ................................................... 121 
Figure 91 FDTD Simulated Gain of Sierpinski 2

Figure 92 FDTD Simulated Constant Phi Gain of Sierpinski 2 _............................... 123

Figure 93 FDTD Run Parameters of Sierpinski 3 …................................................ 124

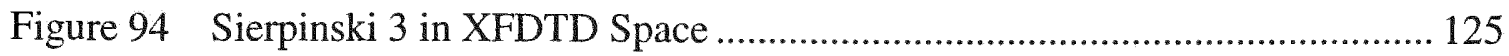

Figure 95 Input Impedance of Sierpinski 3 …...................................................... 126

Figure 96 Real and Imaginary Input Impedance of Sierpinski 3 ............................ 127

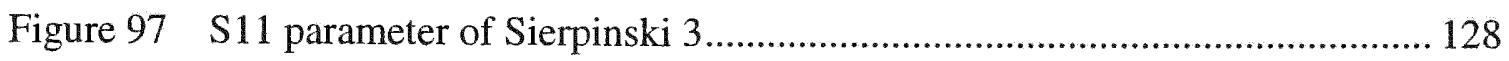

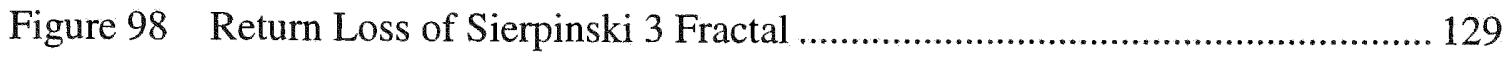

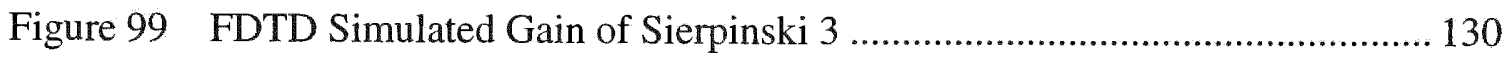

Figure 100 FDTD Simulated Constant Phi Gain of Seirpinski 3 ............................... 131

Figure 101 Return Loss of Sierpinski Antenna with various fractal Iterations.............. 133

Figure 102 Input Resistance for Sierpinski Fractal at various Iterations...................... 134

Figure 103 Comparison of Return Loss for Bowtie and Sierpinski 3 ........................... 135

Figure 104 Return Loss of Bowtie, Sierpinski and Koch Fractal ................................ 137 


\section{Chapter 1 Introduction}

\subsection{Overview}

Due to the huge growth of the mobile telephony and wireless communication, the market is moving towards the integration of as many services as possible. The key to this process of the integration of many services leads to a consideration of multifrequency solutions which respect the environment.

The market penetration of the handheld devices throughout the world has allowed humanity to live in a wireless atmosphere. However, new challenges appear in terms of minimizing the terminal size, such as the need in which to integrate as many systems as possible in a single handset [1].

The combination of mobility and wireless technology is transforming the way in which we communicate, and this is putting new demands on radio-frequency components such as antennas. The size and performance of antennas are very important to satisfy the needs of wireless devices and services.

Antennas for the next generation of cell phones and other wireless communication devices are becoming of great interest to antenna designers. The challenge they face is to design and provide a smaller and more efficient antenna to meet the requirements of the more sophisticated technology in new cell phones, mobile communication and other PDA devices.

These antennas must be very small and they must be able to operate and perform at different frequency band simultaneously. In this sense, Fractal Antennas are the best solutions to accomplish this goal. Antennas are basically narrowband devices. 
Their behavior is very much dependent on the antenna size to the operating wavelength ratio. That is, for a fixed antenna size, the main antenna parameters such as the gain, input impedance, pattern shape and secondary lobe level and distribution, will undergo strong variations when changing the operating frequency [1]. In addition, given a specific frequency, the antenna cannot be made randomly small; it typically has to keep a minimum size, normally in the order of a quarter wavelength.

There is a trade-off in designing small, effective antennas since small antennas are in general poor radiators. One main factor that affects the size of the tags is the frequency that is used.

These known facts have been constraining the antenna performance in telecommunications systems for decades. The size to wavelength dependence is still a problem in many systems where former antenna designs are not particularly suitable [1]. In that sense, the fractal-shaped antennas can help in dealing with the problem by contributing with a vast, rich variety of geometrical shapes with some astounding properties.

Traditional antenna design needs a single antenna for each application. Using fractal technology, this constraint is no longer valid. Fractal Antennas appear to be an attractive way to design multiband and miniature antennas. Antenna Design can benefit from the study of fractal geometries. When applied to antenna design fractals can minimize the size of an antenna and also improve on its impedance matching.

In this thesis project, the research is intended to propose fractal antennas structure for applications in wireless communication. 
The main goal of this research is to study and improve on conventional antennas by applying the concept of fractals to different antennas and using the Finite Difference Time Domain technique to simulate and analyze these structures.

Fractal antennas represent a class of radiators where the overall structure is comprised of a series of repetitions of a single geometry basis, where each geometric repetition is typically on a different scale [2]. It has been shown that when a fractal antenna is of the same overall size as a Euclidean antenna, the fractal antenna has a lower resonant frequency and it exhibits resonance compression of multiband behavior as a function of frequency [2]. Therefore, fractal antennas can be implemented to operate effectively at various frequencies, thus providing a wider band.

In carrying out this research, each proposed antenna structure will first be manually design and the necessary parameters calculated. Each designed structure will be then implemented on the XFDTD platform and simulated. For each structure, the results will be calculated and plot, the performance of each structure will then be observed and compared.

This thesis is based on the properties of fractals when applied to antenna design such as miniaturizing and developing multi-band and wideband antennas.

The two main fractals that will be considered in this thesis research are: Koch Snowflake and the Sierpinski Gasket. These fractals geometry exhibit the characteristics and therefore are investigated and analyzed by using the XFDTD numerical method. The results are observed and compared to a conventional Bowtie antenna with the same parameters. 


\section{Chapter 2 Finite Difference Time Domain (FDTD) Method}

The Finite Difference Time Domain method is by means a very useful numerical method of calculating electromagnetic propagation in the time domain. This method is very useful when applied in solving many electromagnetic related problems. It has emerged as a very reliable and accurate numerical tool in solving Maxwell's equations in the time domain and also being a very convenient method for solving radiation, interaction and scattering problems [3].

\subsection{Introduction}

The FDTD method was first created by Kane S Yee in 1966 [3]-[4]. He developed a three dimensional central finite difference algorithm that solves Maxwell's curl equation in both time and space. FDTD technique is based upon a transient marching in time approach [3] in which the transient fields are computed as a function of time.

Finite Difference Time Domain is a method that divides time into discrete steps and in each time step, electric and magnetic fields are calculated on a discrete grid. This technique is becoming increasingly popular due to it's usefulness in a large variety of electromagnetic problems, including the modeling and analysis of various antenna designs.

The Finite Difference Time Domain (XFDTD) software is marketed by REMCOM, Inc. This software uses the FDTD method for electromagnetic calculations which is an excellent tool for modeling and simulating various electromagnetic problems 
such as antenna radiation scattering/penetration biological calculations and microwave circuit calculations [4].

The FDTD Method has been used extensively for more complex structures and materials than can be accommodated using integral equation approaches. It also provides the advantage of being a time domain technique thereby allowing broadband analysis in a single calculation. This is important for impulse or broadband applications where the use of a frequency domain method would require many calculations to cover the necessary bandwidth.

\subsection{Features and Limitations of XFDTD}

When using the FDTD method there are several useful features and capabilities in which to consider [4].

These features and limitations are:

- Modeling of lossy dielectric and perfect conductor.

- Building, displaying and modifying meshed geometries cell by cell graphically with mouse control.

- The use of local grids to mesh objects with different cell sizes in the same FDTD space.

- Simulations that include dielectric materials with frequency dependant constituent parameters.

- Merging several meshed geometries with material priority control over over-lapping regions. 
- Modeling of thin wires with wire radii smaller than FDTD cell dimensions.

- Choice of excitation pulse (gaussian, gaussian derivative, modulated gaussian, sinusoidal, or user defined).

- Sampling of near zone electric fields and currents.

- Graphical display of near zone fields and currents for each time step over planar slices of the FDTD space.

- Calculation of antenna impedance.

- Transient far zone field calculations as a function of angle for either a constant phi or constant theta pattern.

\subsection{The limitations of XFDTD are:}

- Inability to display electric fields with color-coded positive and negative polarities.

- Limited number of materials types that call for the redesigning of resistive antenna geometries. 


\subsection{FDTD Equations}

The FDTD algorithm was formulated by J.C. Maxwell in 19th century to describe electric and magnetic fields. The algorithms are derived from 4 previous laws as shown below:

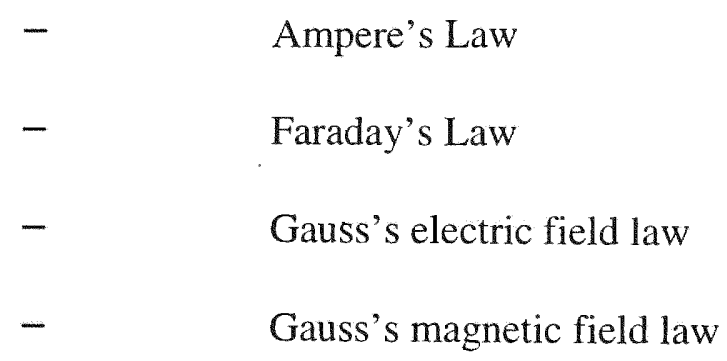

The XFDTD algorithm provides means to numerically solve Maxwell's equations in the time domain [5]. This method is based on the two Maxwell curl equations. The algorithm begins by discretizing Maxwell's curl equations in space and time, thus resulting in two finite-difference equations. FDTD solves differential form of Maxwell's interpretation of Ampere's Law and Faraday's Law.

\subsection{Maxwell's Equation Faraday's Law:}

$$
\begin{aligned}
\frac{\partial \vec{B}}{\partial t} & =-\nabla \times \vec{E}-\vec{J}_{m} \\
\vec{B} & =\mu \vec{H} \\
\vec{J}_{m} & =\rho \vec{H} \\
\nabla \times \vec{E} & =-\mu \frac{\partial \vec{H}}{\partial t}-\rho \vec{H}
\end{aligned}
$$

Where, $\vec{J}_{m}$ is current density, and $\vec{B}$ is magnetic flux density. 
2.6 Scalar Equation:

$$
\begin{gathered}
\frac{\partial H_{x}}{\partial t}=\frac{1}{\mu_{x}}\left(\frac{\partial E_{y}}{\partial z}-\frac{\partial E_{z}}{\partial y}-\rho_{x} H_{x}\right) \\
\frac{\partial H_{y}}{\partial t}=\frac{1}{\mu_{y}}\left(\frac{\partial E_{z}}{\partial x}-\frac{\partial E_{x}}{\partial z}-\rho_{y} H_{y}\right) \\
\frac{\partial H_{z}}{\partial t}=\frac{1}{\mu_{z}}\left(\frac{\partial E_{x}}{\partial y}-\frac{\partial E_{y}}{\partial x}-\rho_{z} H_{z}\right)
\end{gathered}
$$

\subsection{Maxwell's Equation Ampere's Law:}

$$
\begin{aligned}
\frac{\partial \vec{D}}{\partial t} & =\nabla \times \vec{H}-\vec{J}_{c} \\
\vec{D} & =\varepsilon \vec{E} \\
\vec{J}_{c} & =\sigma \vec{E} \\
\nabla \times \vec{H} & =\varepsilon \frac{\partial \vec{E}}{\partial t}+\sigma \vec{E}
\end{aligned}
$$

\subsection{Scalar Equation:}

$$
\begin{aligned}
& \frac{\partial E_{x}}{\partial t}=\frac{1}{\varepsilon_{x}}\left(-\frac{\partial H_{y}}{\partial z}+\frac{\partial H_{z}}{\partial y}-\sigma_{x} E_{x}\right) \\
& \frac{\partial E_{y}}{\partial t}=\frac{1}{\varepsilon_{y}}\left(-\frac{\partial H_{z}}{\partial x}+\frac{\partial H_{x}}{\partial z}-\sigma_{y} E_{y}\right) \\
& \frac{\partial E_{z}}{\partial t}=\frac{1}{\varepsilon_{z}}\left(-\frac{\partial H_{x}}{\partial y}+\frac{\partial H_{y}}{\partial x}-\sigma_{z} E z\right)
\end{aligned}
$$

Where $\mu$ is the permeability, $\varepsilon$ is the permittivity and $\sigma$ is the conductivity of the isotropic material. 


\subsection{Computer Requirements}

It is very important to know that when running the FDTD platform, one should consider the computer resources available. There are two important parameters along with the computers storage capacity that is required for an FDTD simulation.

The computer requirements by the XFDTD program can be determined by considering the following factors. They are: the cell size and the time step.

\subsection{FDTD Cell Size}

It is very important to know that the first step in the geometry design process is to define the size of the FDTD cell.

It should be noted that the cell size should be very small for a very accurate result. The FDTD method requires that the cell size (in $x, y$, and $z$ directions) should be much less than the smallest wavelength of interest.

The typical rule is 10 cells per wavelength, that is, each cell side should be $1 / 10 \lambda$ or smaller at the smallest wavelength or highest frequency of interest.

The size of the cells is determined by:

$$
1 / 10 \lambda \geq \Delta \mathrm{x}, \Delta \mathrm{y}, \Delta \mathrm{z}
$$

Where $\lambda$ is the shortest wavelength been considered.

If the computational problem involves a penetrable material, then the wavelength in the material should be considered since the material conductivity and permittivity affect the wavelength [4]. 


\subsection{The Time Step Size}

Once the FDTD space and cell sizes are determined, then the maximum time-step $\Delta t$ can be calculated from the FDTD space grid. The maximum time step $\Delta t$ needed for stability can be found by using the Courant condition [4].

For a 3-D grid the Courant condition is:

$$
\text { Courant equation }=v \Delta t \leq \frac{1}{\sqrt{\frac{1}{(\Delta x)^{2}}+\frac{1}{(\Delta y)^{2}}+\frac{1}{(\Delta z)^{2}}}}
$$

Where:

$v$ is the velocity of the electromagnetic wave in the medium,

$\Delta t$ is the time step and $\Delta x, \Delta y, \Delta z$ are the dimensions of the cell.

If by any circumstance the FDTD space contains a dielectric material, then $\Delta t$ would need to be lowered proportionally to ensure that the sampling is sufficient.

Therefore, $\Delta t$ decreases by the following:

$$
v \Delta t=\Delta t(\text { free-space }) / \sqrt{\varepsilon_{\mathrm{r}}}
$$

$\varepsilon_{\mathrm{r}}$ is the relative permittivity of the dielectric material. 


\subsection{Specification of the Incident Field}

It should be noted that one of the main advantages in using the scattered field formulation is that the incident field is specified analytically. This can be seen if we assumed our specified incident field is a Gaussian pulse wave. This wave gives the smoothest slope in frequency and thus is best to implement.

A plane wave is introduced by specifying the direction that the incident plane wave is coming from by specifying the angles $\theta$ and $\varphi$, where $\theta$ is measured from the $z$ axis and $\varphi$ is measured from the $\mathrm{x}$-axis.

One can consider that the incident plane wave is in the time domain and are in a spherical coordinate system with the FDTD Cartesian coordinate system. Where $\theta$ and $\varphi$ are the spherical coordinate system unit vectors, $c$ is the speed of light, $\eta$ is the impedance of free space and $\mathrm{r}$ is unit vector pointing from the origin in the FDTD space in which the incident field is computed. 


\subsection{FDTD Problem Space}

Once the cell size has been found, the FDTD problem space can be determined by realizing the number of cells required to design the object plus an appropriate amount of free space.

The total number of cells in the FDTD space is the product of $x, y$, and $z$. In order to obtain accurate results, a margin of 10 to 20 cells in all directions is required to separate the geometry from its outer boundary. The total number of cells depends upon the problem and most computers can accommodate from a few hundred thousand up to several million cells [4].

If the total space were too small for the problem, then the results would be inaccurate. A computational space of $100 \times 100 \times 60$ cells $($ cell size $=1 \mathrm{~cm}$ ) was found to be insufficient for single antenna simulations and was increased to $100 \times 100 \times 100$ cells (cell size $=1 \mathrm{~cm}$ ) for accurate results [4].

Also the absorbing boundary condition $(\mathrm{ABC})$ should be taken into consideration. It is typical to use 10 to 15 cells for the absorbing boundary. These will allow for a more accurate calculation. 


\subsection{Yee Cells}

The Yee's FDTD formulation will be illustrated. In this chapter the Yee FDTD scheme will be restricted to a model with linear, non-dispersive and non-magnetic dielectric. Various considerations such as numerical dispersion, stability of the model and terminating the model with absorbing boundary condition $(\mathrm{ABC})$ will be discussed.

The Yee algorithm Solves for both the electric and magnetic fields in time space. It uses time-dependent scalar equations. Every E component is surrounded by four $\mathrm{H}$ components, and vice-versa, which is useful in boundary situations [5]. The Yee algorithm is also used in time and frequency solutions solved at same time.

This method came to be known as FDTD in which its original work was for lossless materials [5]. In using this algorithm in the FDTD platform we define all points of mesh such as the Dielectric, Magnetic materials and Metal conductors. The E and $\mathrm{H}$ fields are determined everywhere in mesh at every time step. This allows picture of EM field progression throughout structure solving simultaneous equations and matrix inversion are avoided entirely in FDTD, thus greatly reduces computation time. 
The Finite Difference Time Domain method is a numerical method defined for linear isotropic materials. The FDTD problem space is constructed using Yee Cells [5]. The Yee Cell is a 3-dimensional cube in which the permittivity, conductivity and permeability of the material is bounded by the perimeter of the cube. The direction of the electric and magnetic fields are defined with the cell as shown with by the position and direction of the arrows.

The entire problem space is quantized by the unit Yell Cell in the rectangular volume. The materials can be free space, perfect conductor or a lossy dielectric.

The figure below shows the Yee Cell:

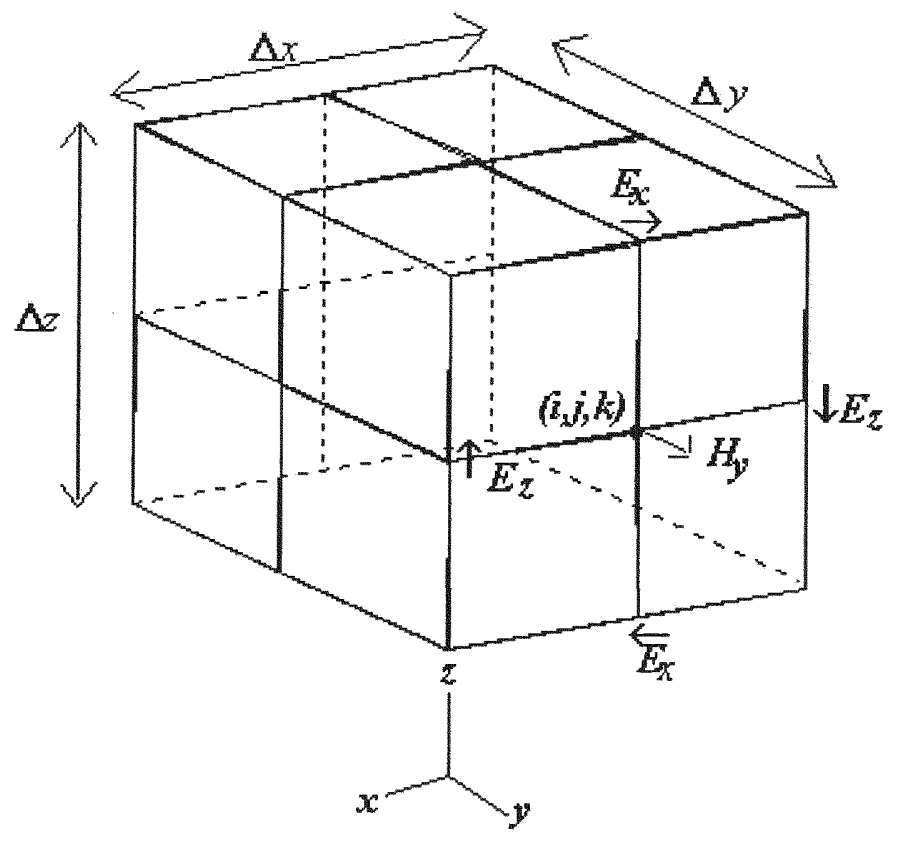

Figure 1 The Yee Cell [5] 
The Yee Cell lattice is assumed to be uniformly in space with each cell edge having length $\Delta x, \Delta y$ and $\Delta z$. The electric and magnetic fields are both in space and time increments. The six field components are found.

The six finite difference equations are:

$$
\begin{aligned}
& E_{x}^{n+1}(i+1 / 2, j, k)=A_{i+1 / 2, j, k} E_{x}^{n}(i+1 / 2, j, k) \\
& +B_{i+1 / 2, j k}\left[H_{z}^{n+1 / 2}(i+1 / 2, j+1 / 2, k)-H_{z}^{n+1 / 2}(i+1 / 2, j-1 / 2, k)\right. \\
& \left.+H_{y}{ }^{n+1 / 2}(i+1 / 2, j, k-1 / 2)-H_{y}{ }^{n+1 / 2}(i+1 / 2, j, k+1 / 2)\right] \\
& E_{y}^{n+1}\left(I_{2} j+1 / 2, k\right)=A_{i, j+1 / 2, k} E_{y}^{n}\left(I_{y} j+1 / 2, k\right) \\
& +B_{i, j+1 / 2, k}\left[H_{x}^{n+1 / 2}(I, j+1 / 2, k+1 / 2)-H_{x}^{n+1 / 2}(I, j+1 / 2, k-1 / 2)\right. \\
& \left.+H_{z}{ }^{n+1 / 2}(i-1 / 2, j+1 / 2, k)-H_{z}^{n+1 / 2}(i+1 / 2, j+1 / 2, k)\right] \\
& E_{z}^{n+1}(i, j, k+1 / 2)=A_{i, j, k+1 / 2} E_{z}^{n}(i, j, k+1 / 2) \\
& +B_{i, j, k+1 / 2}\left[H_{y}{ }^{n+1 / 2}(i+1 / 2, j, k+1 / 2)-H_{y}{ }^{n+1 / 2}(i-1 / 2, j, k+1 / 2)\right. \\
& \left.+H_{x}^{n+1 / 2}(i, j-1 / 2, k+1 / 2)-H_{x}^{n+1 / 2}(i, j+1 / 2, k+1 / 2)\right] \\
& H_{x}^{n+1 / 2}(i, j+1 / 2, k+1 / 2)=H_{x}^{n-1 / 2}(i, j+1 / 2, k+1 / 2) \\
& +\Delta t / \mu \delta\left[E_{y}{ }^{n}(i, j+1 / 2, k+1)-E_{y}{ }^{n}(i, j+1 / 2, k)\right. \\
& \left.+E_{z}^{n}(i, j, k+1 / 2)-E_{z}^{n}(i, j+1, k+1 / 2)\right] \\
& H_{y}{ }^{n+1 / 2}(i+1 / 2, j, k+1 / 2)=H_{y}{ }^{n-1 / 2}(i+1 / 2, j, k+1 / 2) \\
& \Delta t / \mu \delta\left[E_{z}^{n}(i+1, j, k+1 / 2)-E_{z}^{n}(i, j, k+1 / 2)\right. \\
& \left.+E_{x}^{n}(i+1 / 2, j, k)-E_{x}^{n}(i+1 / 2, j, k+1)\right] \\
& H_{z}^{n+1 / 2}(i+1 / 2, j+1 / 2, k)=H_{z}^{n-1 / 2}(i+1 / 2, j+1 / 2, k) \\
& \Delta t / \mu \delta\left[E_{x}^{n}(i+1 / 2, j+1, k)-E_{x}^{n}(i+1 / 2, j, k)\right. \\
& \left.+E_{y}{ }^{n}(i, j+1 / 2, k)-E_{y}{ }^{n}(i+1, j+1 / 2, k)\right]
\end{aligned}
$$




\subsection{Absorbing Boundary Condition}

Types of boundary conditions

- $\quad$ Mur's first and second order $\mathrm{ABC}[6]$

Some important considerations about the boundary condition are shown below:

On metal surfaces, tangential electric fields are set equal to zero.

Average dielectric value at boundaries

Simulation lasts forever without boundaries

Boundary shouldn't be near the circuit.

In modeling a structure that is in free space, such as a radiating antenna or a scattering object, we would want the radiated or scattered fields to propagate into boundless free space without the aid of an absorbing boundary [6]. The absorbing boundary condition $(\mathrm{ABC})$ thus, provides and simulates the effect of free space. If the absorbing boundary condition is not set correctly, then the FDTD formulas will not be able to use the correct values to update the fields. This will result in the field being reflected on the boundaries other than propagating outwards.

It should be noted that the boundary condition in the FDTD is a second order stabilized radiation boundary [6]. There is a minimum free space that should be taken in account when placing a scattering object with the FDTD space. This minimum free space between the scattering object and the outer boundary should be 10 to 15 cells or more for the best results. 


\subsection{XDTD Validation Process}

The XFDTD platform as stated previously is a numerical method that was developed by Remcom Inc [4] to compute electromagnetic propagation in the time domain. It is used in this thesis as the calculation and simulation tool in our fractal antennas design and simulation.

The FDTD calculation included the simulation project setup, the output data analysis and the geometry definition. We will use and follow this process as shown below to design and analyze fractal antennas used in wireless communications.

Shown below is the FDTD process:

At time $\mathrm{t}=0$, all fields are set equal to zero

The number of time steps $\mathrm{n}$ are chosen

Gaussian excitation started at input port

Electric field values calculated from FDTD equations

Magnetic field values calculated from FDTD equations

The tangential $\mathrm{E}$ fields are set to zero on all metal conductors in the mesh

The field values are saved in holding matrices

Move forward one time step

After the number of time steps have been completed, compute the scattering matrix parameters from the time domain results. 


\section{Chapter 3 Fractals}

\subsection{Introduction to Fractals}

"Fractal" is a word founded by Bonoit Mandelbrot in 1975 to describe shapes which are detailed at all scales [8]. Mandelbrot definition of fractal dimension of a geometric object was based on the definition given by Hausdrof in 1919. This definition involves a limit process. It is basically a change in the object size versus the change in measurement scale, as the measurement scale approaches zero. Logarithms are used for both size and scale.

The word Fractal is originated from the Latin root "Fractus" which implies fragmented, broken and discontinues [8]. Fractal can be looked at as a rough or fragmented geometric shape that can be subdivided in parts, each of which is a reduced size copy of the whole [8].

From a mathematical view, fractal is a set for which the Hausdroff-Besicovitch dimension strictly exceeds the topological dimension. This Hausdroff- Besicovitch dimension is called "Fractional Dimension" [9]; they may also be defined recursively.

Also, a fractal can be classified as an object which is self-similar; they are repetitive in shape, but not in size. That is, no matter how much you magnify a fractal it will always look the same or at least similar. 


\subsection{What are Fractals?}

Fractal geometry is the geometry of the irregular shapes that can be found in nature and in general fractals are characterized by infinite detail, infinite lengths and the absence of derivative or smoothness [8]-[9]. They can be used to generate landscapes or sunset, mountains, ripples on lakes and rough terrains, clouds and coastlines that do not correspond to simple geometric shapes.

Fractals, short for "fractional dimension," are mathematical models originally used to measure jagged contours such as coastlines [9]. Like a mountain range whose profile appears equally rocky when observed from both far and near, fractals are used to define curves and surfaces, independent of their scale [9]. Any portion of the curve, when enlarged, appears identical to the whole curve a property known as "self-symmetry." Most fractal shapes have self-similar shapes, although a few can be found that does not posses this character. These fractal shaped objects have infinite complexity, that is, the shaped of the fractal remains no matter how far you zoom-in.

There are many mathematical structures that are fractals; eg. Von Koch snowflake, Sierpinski gasket, the Mandelbrot set, Cantors comb and the Lorenz attractor, et.

For example Mandelbrot [8] discuss the fractal theory in nature as it relates to the characteristics of a coastline (Figure 2). It is seen that the length of the coastline depends greatly on the size of the measuring yardstick/ruler. 
Considering the stretch of coastline, we can see that the length is at least equal to the distance measured along a straight line between its beginning and its end [9]. On the other hand, a typical coastline is irregular and winding and there is no uncertainty that it is much longer than the straight line between its end points. There is a self-similar trait seen as we observe the coastline at various resolutions.

With a fractal object, like a coastline, the smaller you make your ruler, the longer the coastline appears. This is because smaller and smaller rulers measure smaller and smaller jigs and jags in the coastline. Fractal objects have jigs and jags on all scales. They do not start to look smooth as you magnify them [9].

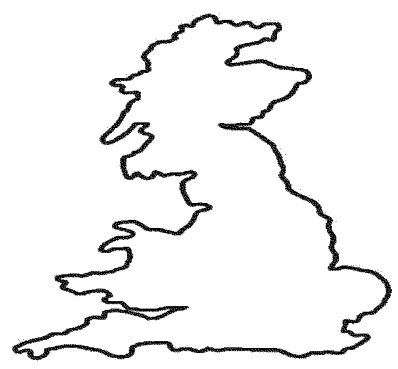

Figure 2 Coastline [9] 
Another example in which fractal geometries are present in nature can be seen in a fern, as shown in Figure 3, the entire leaf has the same structure as each branch. It is seen that if each individual branches were looked at very closely, it is very hard to imagine that it is a completely separate leaf with branched if it's own.

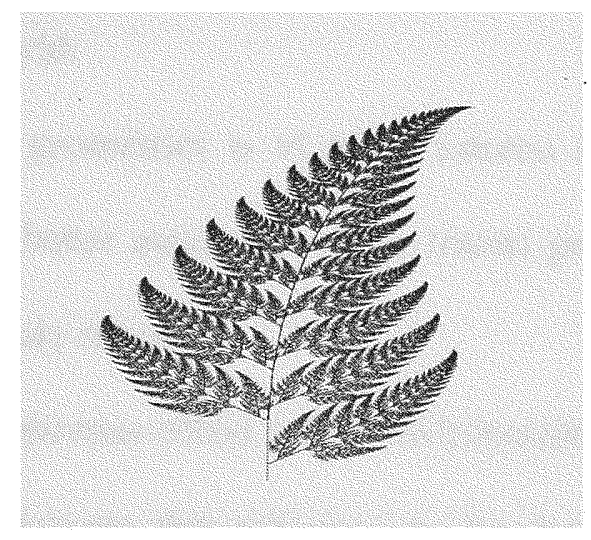

Figure 3 Fern [9]

With these known properties of fractal structures, we will now focus on fractal being applied to antenna design. Chapter 4 in this thesis research discusses the application of fractal theory in antenna design. Antenna Design is becoming a very import aspect in applying fractal geometry. This is the main reason why this thesis project was done, to investigate the characteristics and performance of fractals antennas. 


\section{Chapter 4 Fractal Applied to Antenna Design}

\subsection{Introduction}

Fractal geometries and its uses have significantly found its way in many areas of science and engineering. Fractals have captured the attention, enthusiasm and interest of many people around the world.

One area in which fractal geometries is of much interest is antenna design. With the increasing in demands for better antenna designs, fractal geometries have been used in antenna applications relatively recently.

Fractals, when applied to antennas offers a rich environment in which to explore, create and build new antenna structures that will lead to the development of more efficient antennas.

The concept of applying fractals to the development of various antennas allows for smaller, resonant antennas that are multi-band and broadband and have optimized gain. With the ability of most fractals to have infinite complexity and the detail can be used to reduce antenna size and design low profile antennas.

Fractal antennas have drawn the interest in many antennas designer and researchers and have been studied extensively over the last few years. There have been many studies and research of different fractal shaped antenna: such as the Sierpinski gasket, Sierpinski carpet, Koch curve, Koch snowflake, Minkowski patches, Minkowski island and the Fractal tree antenna to name a few. 
In this thesis study, we will be concentrating on two fractal geometries. The Sierpinski gasket, Koch snowflake are explored. Some distinguishing features of their use demonstrated by corresponding antenna characteristics are presented separately.

In this thesis work it is seen that apart from bringing in the necessary mathematical sequence, the use of these fractal geometries can be exploited in antenna design by integrating their fractal definition into conventional antenna design formulae.

\subsection{Fractal Antenna Design}

With the increasing demands for better performing antennas and the advancement of the communication systems and other wireless applications, wideband and low profile antennas are of high demand in the communication industry.

A conventional wideband antenna when designed to operate in the low frequency wireless bands can only be attained with heavily loaded wire antennas, which typically incorporates different antennas for the desired frequency bands [11].

The concept of fractal theory being applied to antenna design comes down to the basics of how an antenna works. In order for an antenna to work effectively at all frequencies it must satisfy two criteria; it must be symmetrical about a point and it must be self similar, having the same basic shape at every scale, that is, it has to be fractal [11]. Also, the size of an antenna is a critical parameter, since the behavior of the antenna depends on its size. That is, the antenna has to have and keep a certain size to operate effectively. If the antenna size is not the correct one, then the antenna performance will be greatly reduced [12]. 
Fractal technology enables us to design miniature and multiband antennas because of its self-similar and space filling properties of fractal shapes. This fractal technology uses the properties of fractal geometries to design effective and advanced antennas. Fractal antennas have the properties to use a single small antenna to be operated in several frequency bands, thus eliminating the need for multiple and large antennas.

A fractal antenna is an antenna that uses a fractal design to maximize the length of material that can receive or transmit electromagnetic signals within a given total surface area. This result in fractal antennas to be very compact and are anticipated to have useful applications in cellular telephone and microwave communications.

It is found that the mathematical principles behind the repetition of these geometrical structures with similar shapes could be applied to a methodology for developing antenna designs [12].

With this method, we can develop antennas that meet two important challenges presented by the new generation of wireless devices. They conserve space and can operate simultaneously at several different frequencies.

Fractal methodology allows you to pack more electrical length into smaller spaces. It allows the antenna size to be significantly reduced without degrading the performance [13]. By increasing the electrical length the antenna can resonate at lower frequencies.

Since fractal designs are self-symmetrical, they are effective in developing antennas that operate at several different frequencies. One section of the antenna can resonate at one frequency while another section resonates at another frequency. 
Fractal antenna response differs greatly from conventional antenna designs, in that it is capable of operating optimally at many different frequencies simultaneously. In general standard antennas have to be designed for the frequency for which they are to be used and thus only optimally work at that frequency. This makes the fractal antenna an excellent design for broadband applications in which an antenna can be designed to operate at different frequencies.

\subsection{Advantages of using Fractals}

The space-filling nature of fractal allows a high response fractal antenna to be fitted into a relatively small space. Also depending on the geometry, antennas can be multi-band with resonant frequencies reflecting the self-similarities of the fractal or alternatively can have frequency independent response.

The use fractals in antennas design when compared to conventional antenna designs center around size and bandwidth. This means that the size of an antenna can be reduced from two to four times with surprising good performance. Mulitband performance is nonharmonic frequencies and at higher frequencies the fractal antenna is naturally broadband [14]. It should also be noted that polarization and phasing of fractal antennas are possible in attaining. 


\subsection{Self-similarity}

When parts of a figure contain small copies of the whole, then the figure is said to be self-similar. If a figure can be broken into parts which are exact copies of the whole, then the figure is said to be strictly self-similar. This notion of self-similarity is the main characteristics of many fractals [15].

Self-similar antennas have been known since Mushiake's 1948 work on selfcomplementary designs [15]. However, it has only been in the last few years that fractal antennas have been extended to a more generalized design approach. The self-similar patterns can be exploited to incorporate one or more useful attributes including: substantial size shrinkage; broad or multi bands; increased gain; power pattern agility.

Self similarity means that the antennas can operate simultaneously in multiple bands with similar radio-electric parameters.

\subsection{Fractal Dimension}

Fractal dimension means that the geometry does not have one, tow or three dimensions like most of the objects we are used to, but they have incomplete dimensions that are not integer values. Unlike Euclidean geometries that have integer dimensions, fractals have fractional dimensions.

Fractal dimension concept has the property that allows us to minimize antennas. An antenna is said to be small when its larger dimension is less than two times the radius of the radian sphere in which the antenna is enclosed.

Because of the space filling properties of some fractal structures, fractal dimension might allow fractal shaped antennas to better take advantage of the small surrounding space [14]. Since fractals are space filling geometries, then they can 
efficiently incorporate large electrical lengths. The dimension of the geometry can be looked at as a quantification of the space filling ability of the geometry.

\subsection{Fractal Antenna Configuration / Method}

In this research two antenna cases have been analyzed and implemented to find the optimum configuration. These fractal antenna configurations were structured and tested using the FDTD numerical method, such as the Koch Snowflake and the Sierpinski gasket.

The main focus in this thesis is to simulate these two fractal structures and analyze there characteristics as an antenna. The antennas were modeled using FDTD, the cell dimensions, cell space step and absorbing boundary conditions and other parameters were found and implemented. Below is a brief description of the two fractal geometries that are studied in this thesis. They will further be discussed in chapters 6 and 7 . 


\subsection{The Sierpinski Triangle/Gasket}

The Sierpinski gasket is named after the Polish mathematician Waclaw Sierpinski who described some of the main properties of this fractal geometry. The Sierpinski triangle has Hausdorff dimension $\log (3) / \log (2) \approx 1.585$, which follows from the fact that it is a union of three copies of itself, each scaled by a factor of $1 / 2$.

The Sierpinski Triangle structure exhibit strict self-similarity feature. This structure naturally decomposes into three triangular parts, an upper portion and two lower portions.

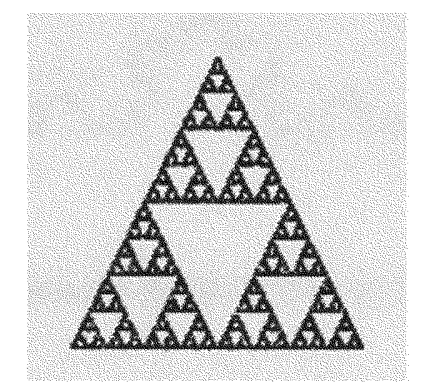

Figure 4 Sierpinski Gasket [23]

Each of these parts is an exact copy of the whole original structure. Each of its three main parts is exactly a scaled version of the original object. Since each and every part of the Sierpinski Triangle has this property it is this feature why it's said to be strictly self-similar.

The self-similarity properties of the fractal shape are translated into its electromagnetic behavior and thus results in creating a multi-band antenna. 


\subsection{Koch Curve}

The Koch curve figure uses a recursive, scaling and substitution process to produce its structure. The Koch curve has been used to reduce antenna sizes. Koch curves exhibit a novel and most interesting combination of complexity and simplicity.

The Koch Curve was found by Helge Von Koch in 1904 [16]. It is built by starting with an equilateral triangle, removing the inner third of each side, building another equilateral triangle at the location where the side was removed, and then repeating the process indefinitely.

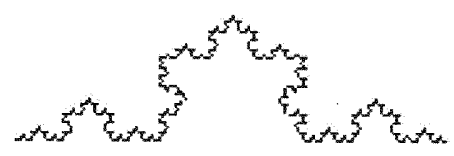

Figure 5 Koch Curve [16]

The Koch curve has infinite length because each time the steps above are performed on each line segment of the figure its length increases by one third. The length at step $n$ will therefore be $(4 / 3)^{\mathrm{n}}$ and the fractal dimension is $\log 4 / \log 3=\sim 1.26$. The Koch curve is continuous, but not differentiable anywhere. 


\section{Chapter 5 Antennas}

\subsection{Introduction}

An antenna can be described as a metallic device such as a rod or wire for radiating or receiving radio waves [1]. The antenna is the transitional structure between free-space and a guiding device. This guiding device is usually a coaxial line or a waveguide which is used to transport electro-magnetic energy from the transmitting source to the antenna or from the antenna to the receiver [1]. An electromagnetic wave consists of changing magnetic field and electric fields and transport through free space.

Conventional antennas are essentially narrowband devices. There are many types of antennas each having different characteristics. The typical types of common antennas are the dipole and the loop antennas. These types of antenna typically have a broad radiation pattern that when positioned vertically will transmit and receive in all directions. These are referred to as an isotropic antenna.

There behavior and performance is greatly dependent on the antenna size to the operating wavelength ratio [1]. That is, for a fixed antenna size the main antenna parameters such as gain, input impedance and pattern shape will go through strong variations when altering the operating frequency.

In this thesis research, we would be studying the characteristics and performance of fractal antenna. However, these fractal shaped geometries where created using a conventional Bowtie antenna. The since a fractal shaped antenna need to be created from a initiator, then using the bowtie antenna and applying the fractal methodology to it results in us creating two fractal shaped antennas. The resulting fractal shaped antennas are: Koch Dipole and Sierpinksi Triangle. 


\subsection{Introduction Bowtie Dipole Antenna}

In this thesis research we will be conducting an investigation on fractal antenna characteristics. This research will take part in three stages. First we will consider a Bowtie Antenna and then we would apply fractal theory to this antenna in designing a Koch fractal and a Sierpinski Fractal.

The reason why the bowtie was considered was because of it similarities of the geometries. The two fractal structures that are considered in this thesis have bowtie geometry. It should be noted that the initiator geometry of the Koch and Sierpinski fractal structure is a bowtie structure.

A dipole antenna consists of two lengths of straight wire fed at its center and looks as an open circuit. The dipole is typically referred to as an antenna whose overall length is one-half wavelength.

The Bowtie dipole antenna is created by taking two equilateral triangles and feeding at its center as shown in Figure 6.

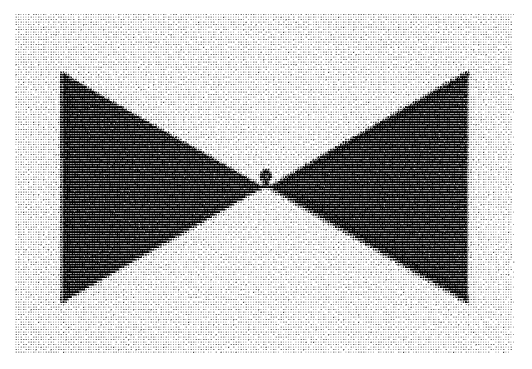

Figure 6 Bowtie Dipole Antenna

The dimension of the Bowtie is $72 \mathrm{~mm}$ for each side of the triangle. 
The length of the bowtie is $123 \mathrm{~mm}$. A $1 \mathrm{~mm}$ feed-point was placed at the center of the two triangles thus forming the bowtie antenna. The geometry dimension is shown in figure 7.

\subsection{XFDTD Analysis of Bowtie Dipole Antenna}

In carrying out this thesis research, we first developed and implement a Bowtie Dipole antenna. Since in this thesis we would be investigating the characteristics of the Koch Snowflake and the Sierpinski Gasket dipole antenna and then compare the results with that of a convention dipole antenna we would need to do a XFDTD numerical analysis of a dipole antenna.

The Bowtie dipole antenna was used in the analysis since it is the same size and geometry as the initiator geometry of the fractal antennas under study. The structure was implemented on the FDTD platform and the simulation was carried out.

The structure was implemented and design using the following parameters as discussed in section 5.4 of this chapter. 


\subsection{Geometry of Bowtie Dipole Antenna}

The Bowtie dipole antenna geometry was created using two equilateral triangles. This was implemented in the XFDTD platform. The dimension of each side of the triangle is $72 \mathrm{~mm}$. The two triangles are fed at the center using a Modulated Gaussian source with pulse width of 768 and input impedance of $50 \Omega$. The Antenna was simulated at amplitude of 1 volts and the computation was run for 4500 time step. The antenna was mounted on a FR-4 substrate with a relative dielectric constant $\varepsilon_{\mathrm{r}}=4.7$. The thickness of the substrate is $0.01 \lambda$. The antenna was modeled using the FDTD model. The 3 dimensional FDTD space was determined. The FDTD computational space has $200 \times 180 \times 20$ cells, $\Delta X=0.05 \mathrm{~mm}, \Delta \mathrm{Y}=0.05 \mathrm{~mm}$ and $\Delta \mathrm{Z}=0.047 \mathrm{~mm}$.

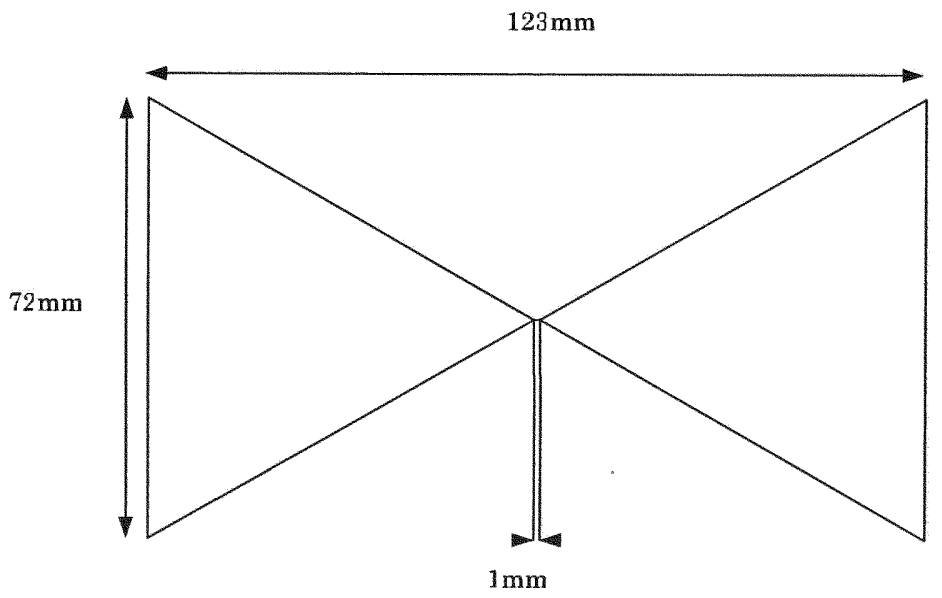

Figure 7 Geometry of Bowtie Dipole Antenna 


\subsection{Results of XFDTD Simulation}

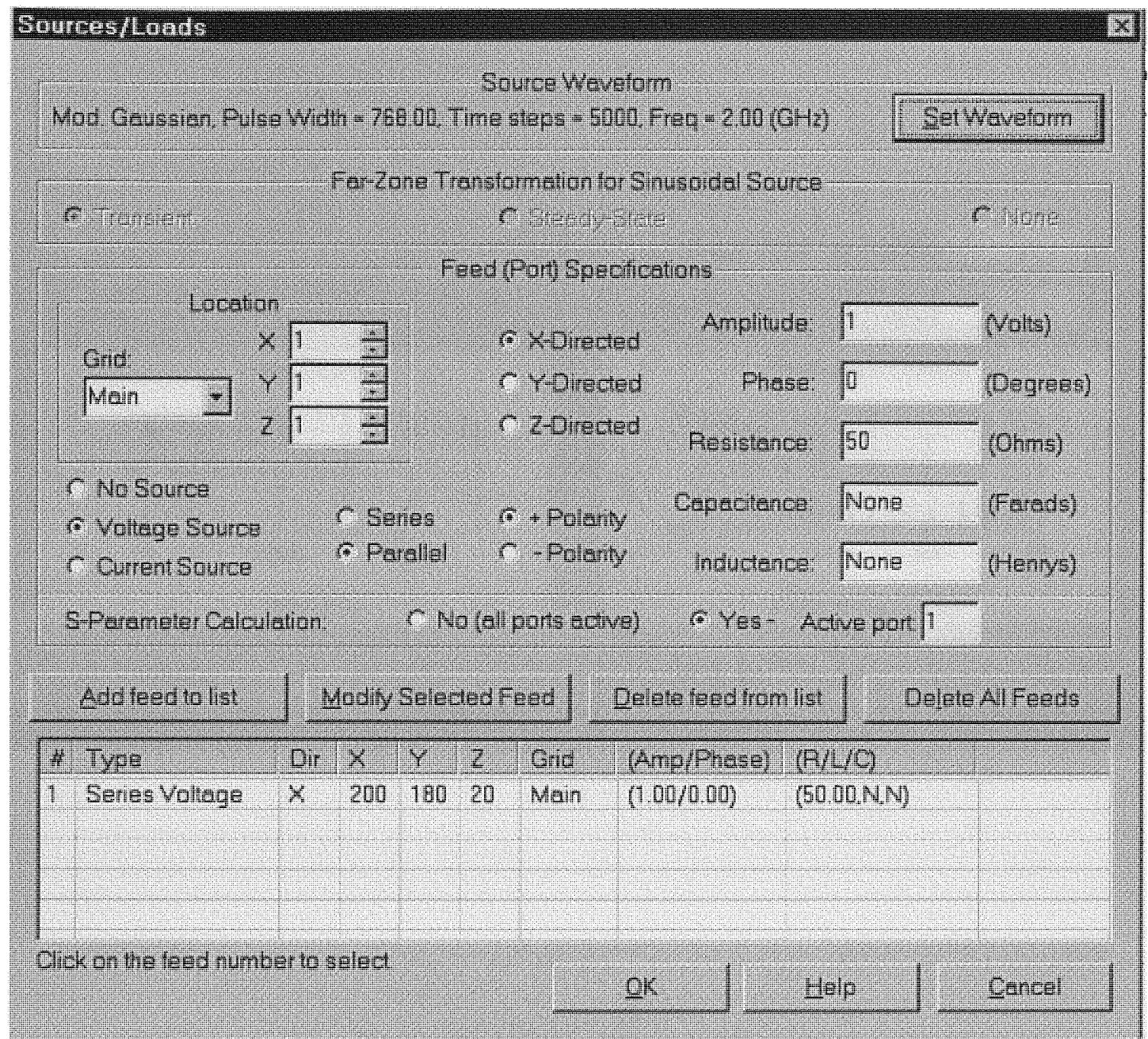

Figure 8 XFTD Source / Loads 


\subsection{XFDTD Stimulus Waveform}

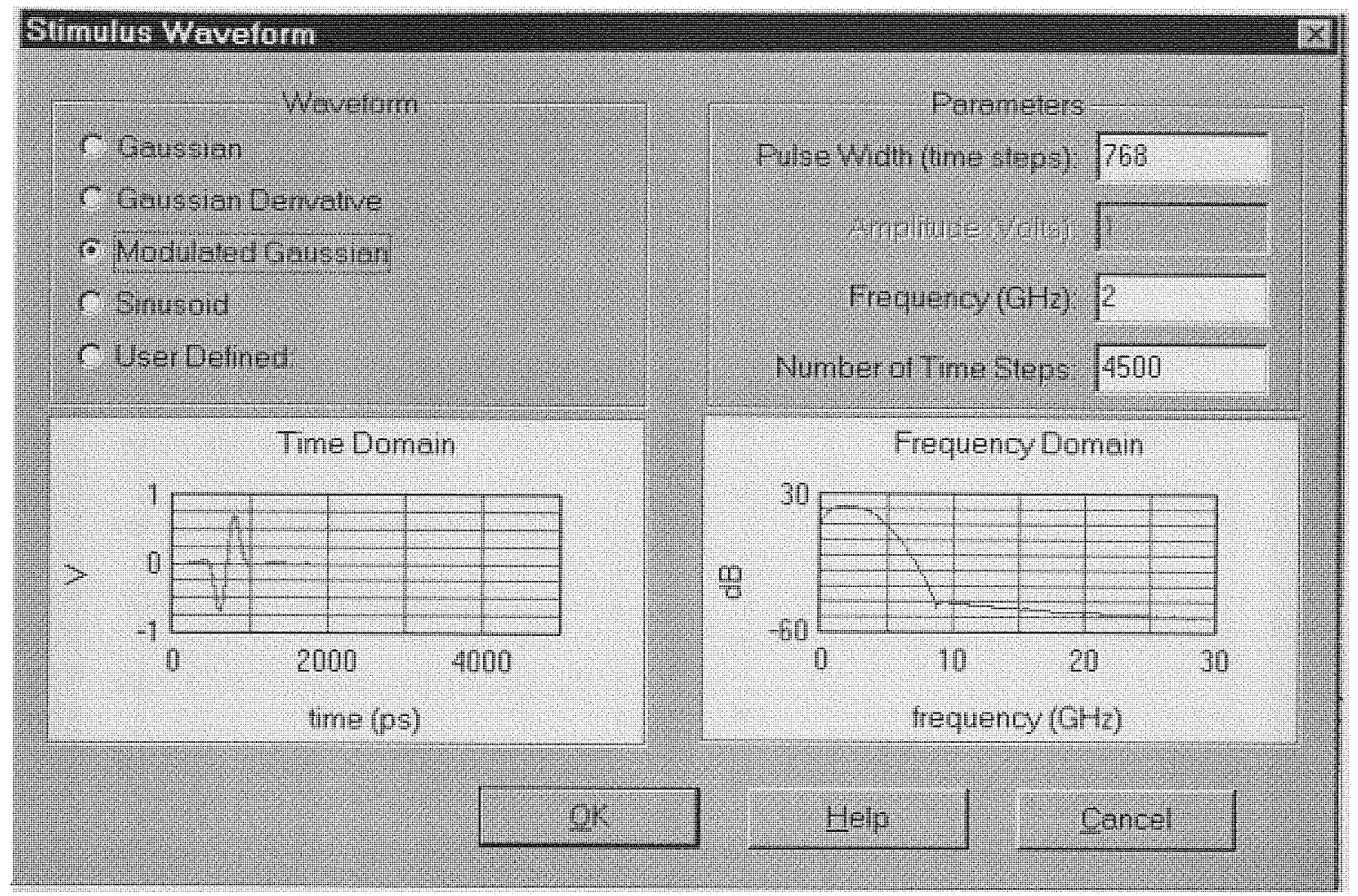

Figure 9 XFDTD Stimulus Waveform 


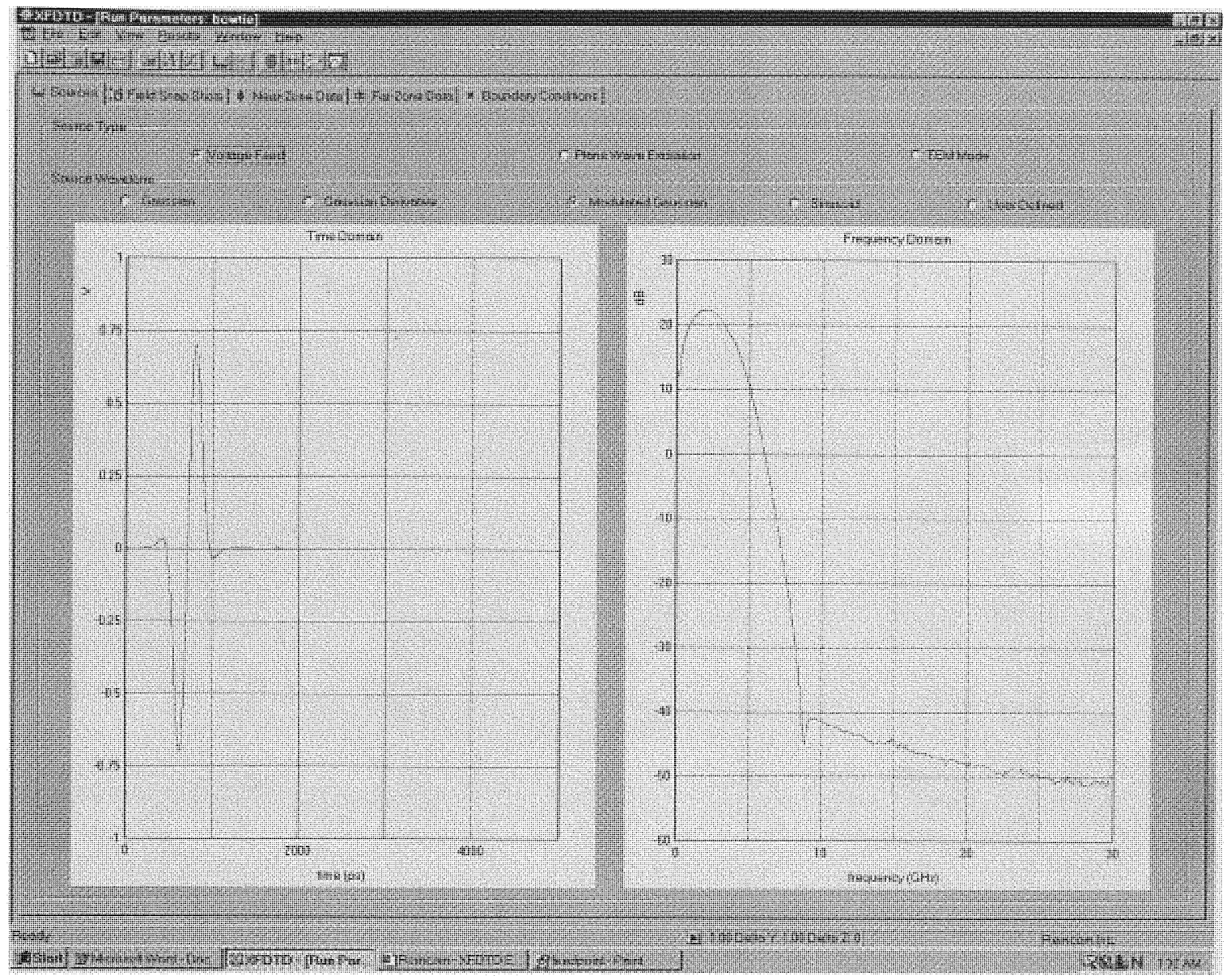

Figure 10 XFDTD Run Parameters

Figure 10 shows the FDTD run parameters. This is the setup of the source parameters used in the FDTD simulation. The Modulated Gaussian was used in this simulation. 


\subsection{XFDTD Space}

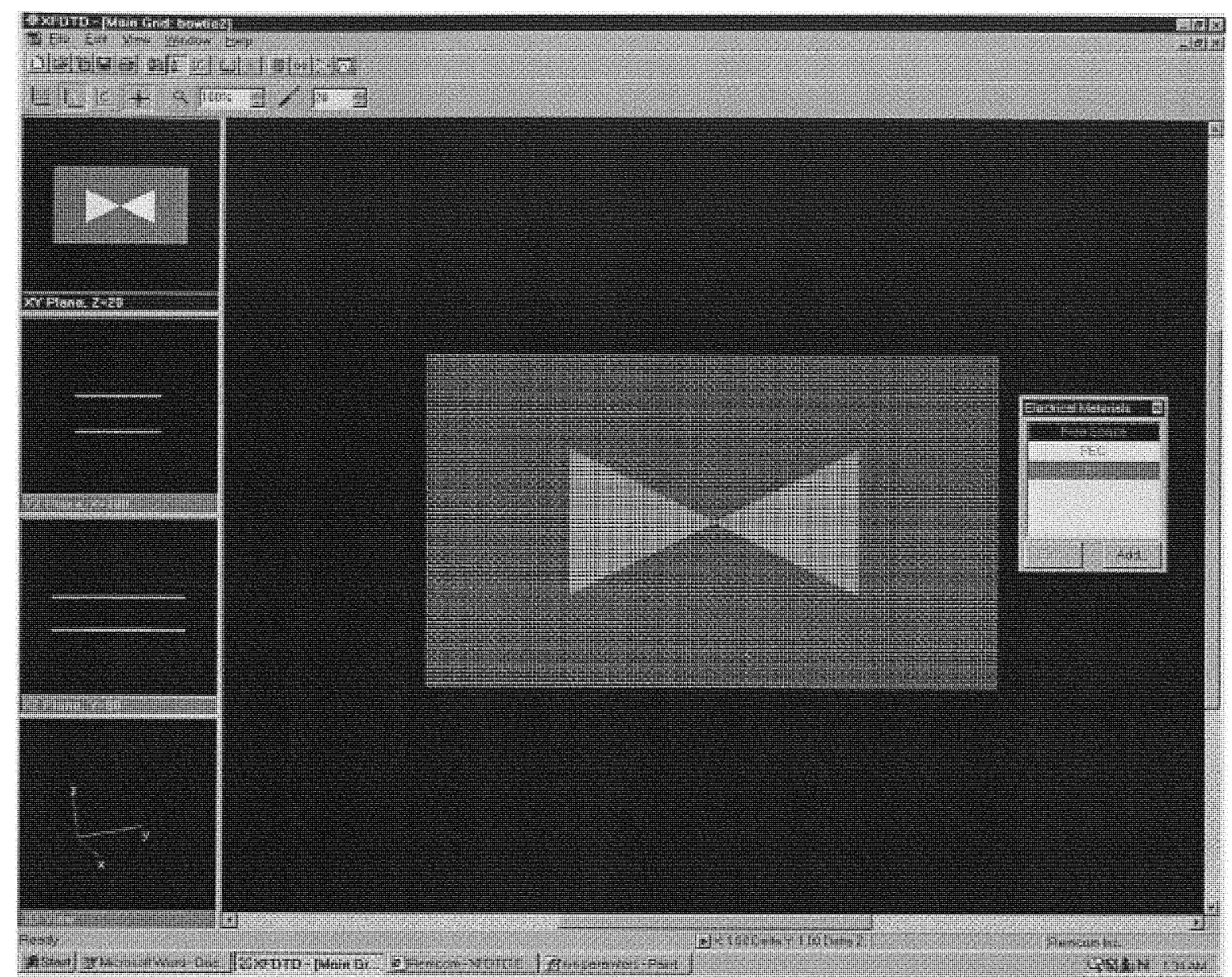

Figure 11 Bowtie Dipole Antenna in XFDTD space 


\subsection{XFDTD Input Voltage vs Frequency}

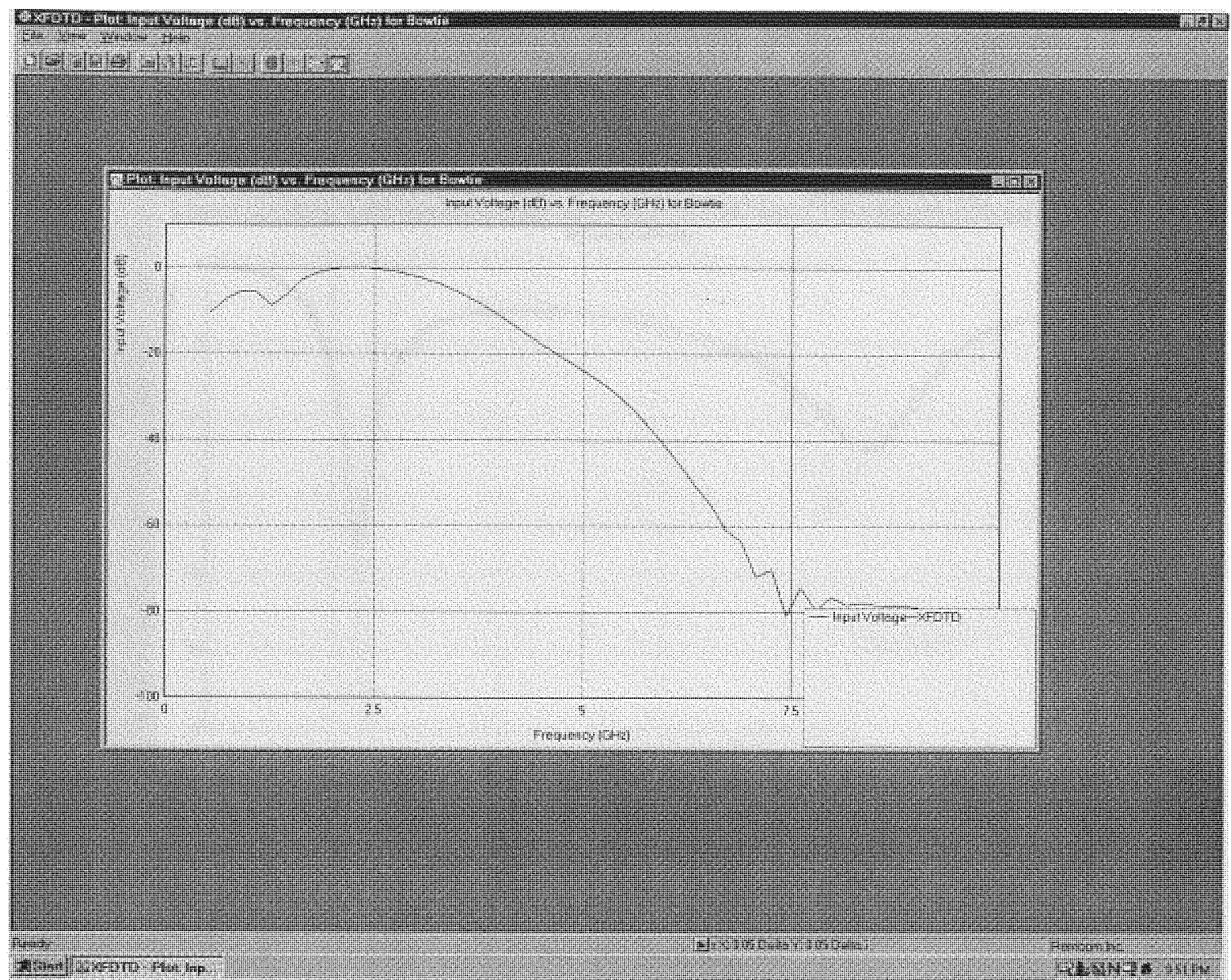

Figure 12 Input Voltage vs Frequency for Bowtie 


\subsection{XFDTD $S_{11}$ Parameters}

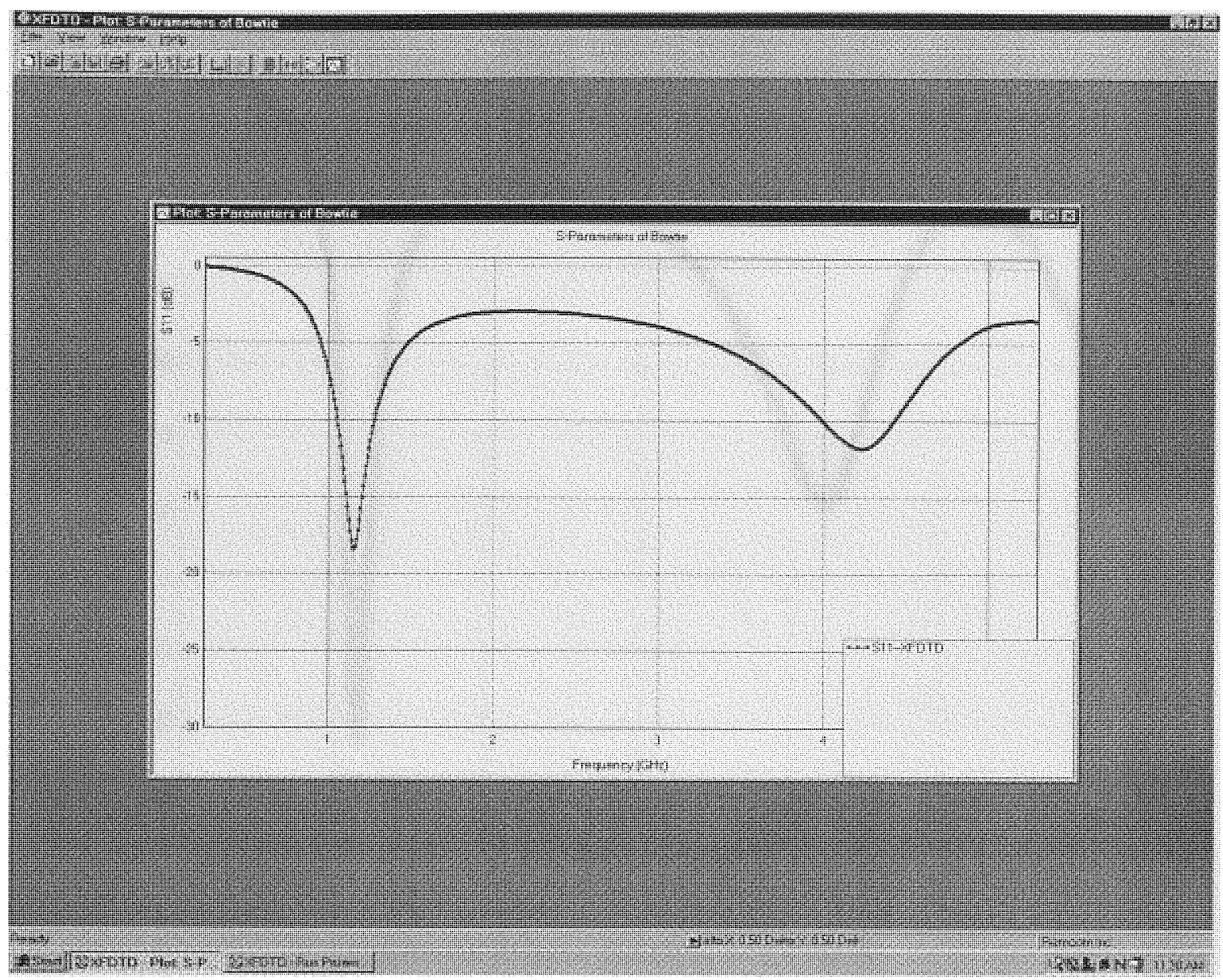

Figure $13 S_{11}$ Parameter for Bowtie Antenna

The S11 simulated results shows that the Bowtie antenna has a resonant frequency of about $1.23 \mathrm{GHz}$. 


\subsection{Return Loss for Bowtie}

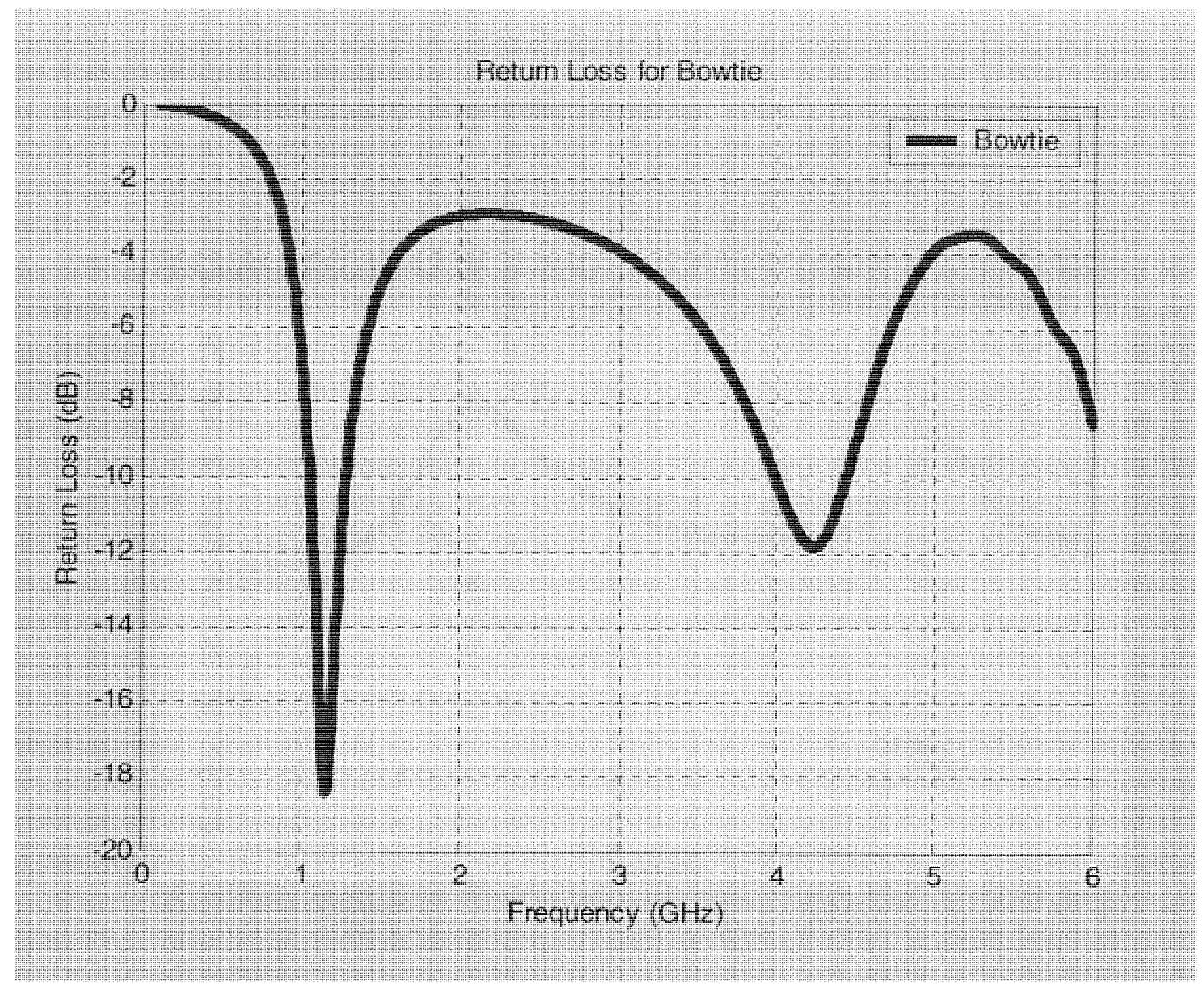

Figure 14 Return loss of Bowtie Dipole

The return loss for bowtie antenna simulated using Matlab. The result corresponds to the simulated results obtained from the FDTD simulation. The input impedance bandwidth obtained from the numerical calculation has an operational bandwidth ranges from about $1.23 \mathrm{GHz}$ to $4.3 \mathrm{GHz}$. At resonant frequency of $1.23 \mathrm{GHz}$ it is found that Bowtie is to be $-19 \mathrm{~dB}$ 


\subsection{XFDTD Input Impedance vs Frequency Bowtie Dipole}

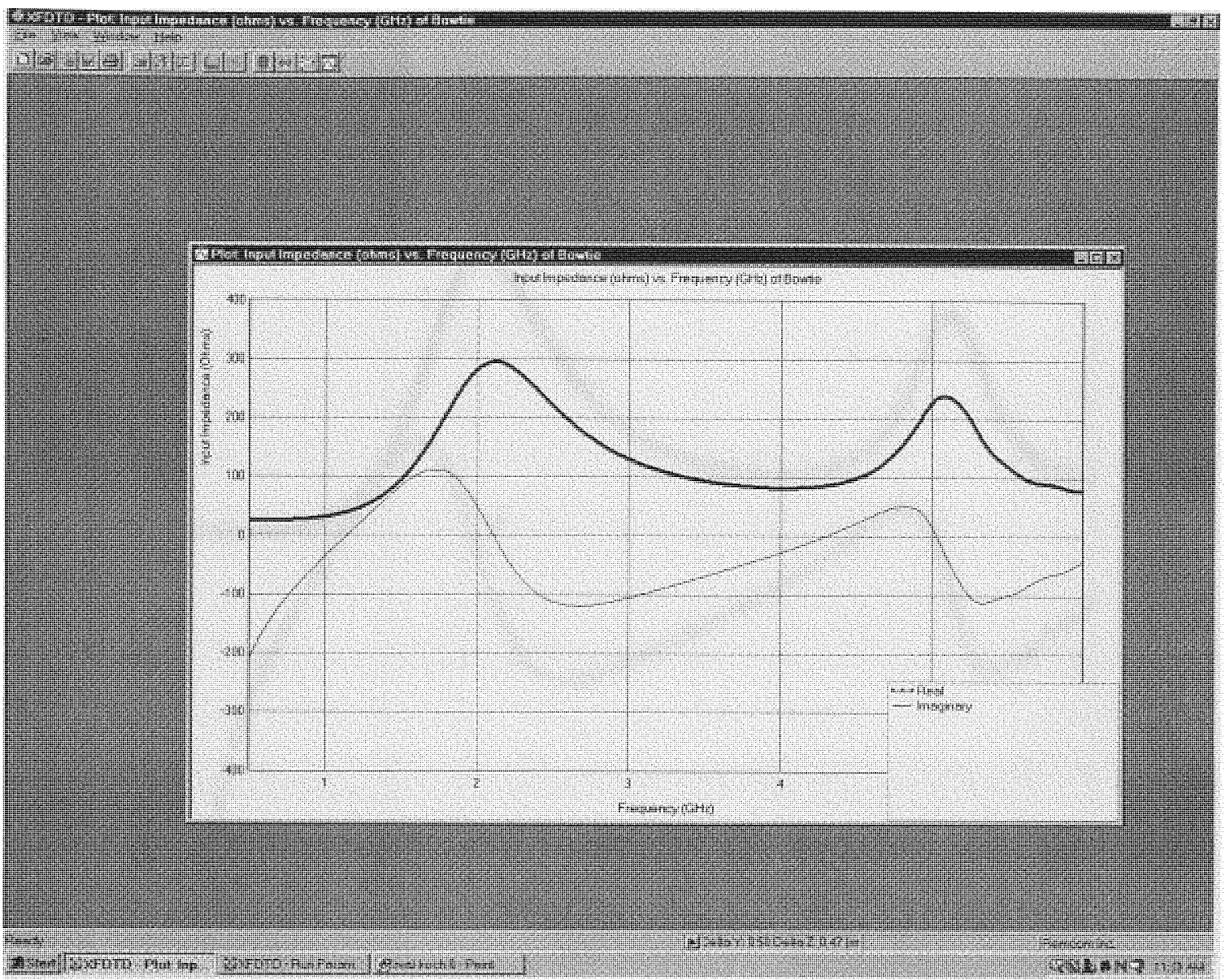

Figure 15 Input Impedance for Bowtie Dipole

The figure above shows the Imaginary and Real part of the Input Impedance of the Bowtie antenna. The results were obtained from the FDTD simulation. 
Figure 16 below shows the Imaginary and Real part of the Input Impedance of the Bowtie antenna.

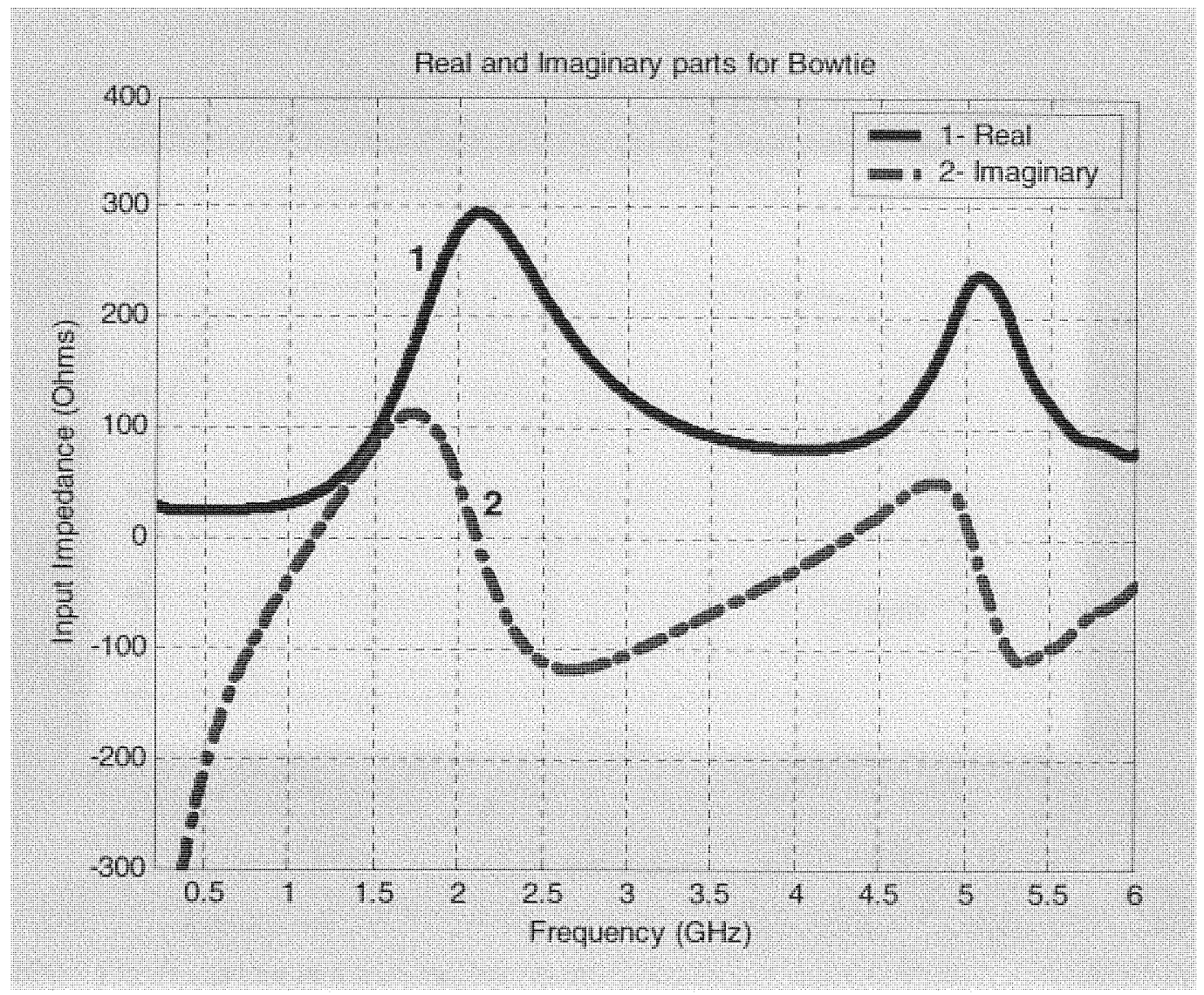

Figure 16 Real and Imaginary Input Impedance for Bowtie Dipole 


\subsection{FDTD Gain vs Theta plot}

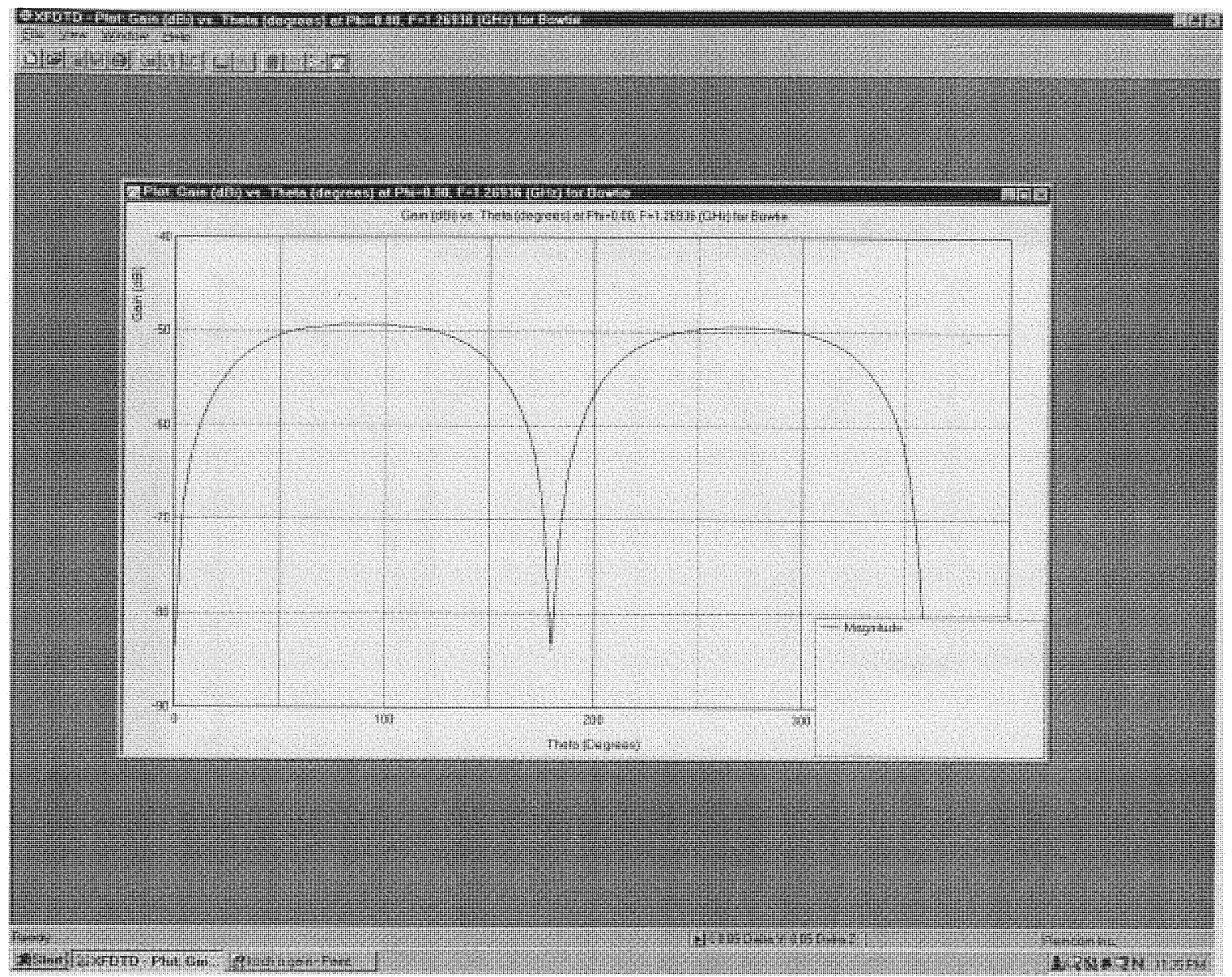

Figure 17 FDTD Simulated Gain of Bowtie Antenna

The above figure shows the Gain vs Angle XFDTD simulation. 


\subsection{FDTD CP Gain vs Theta Plot}

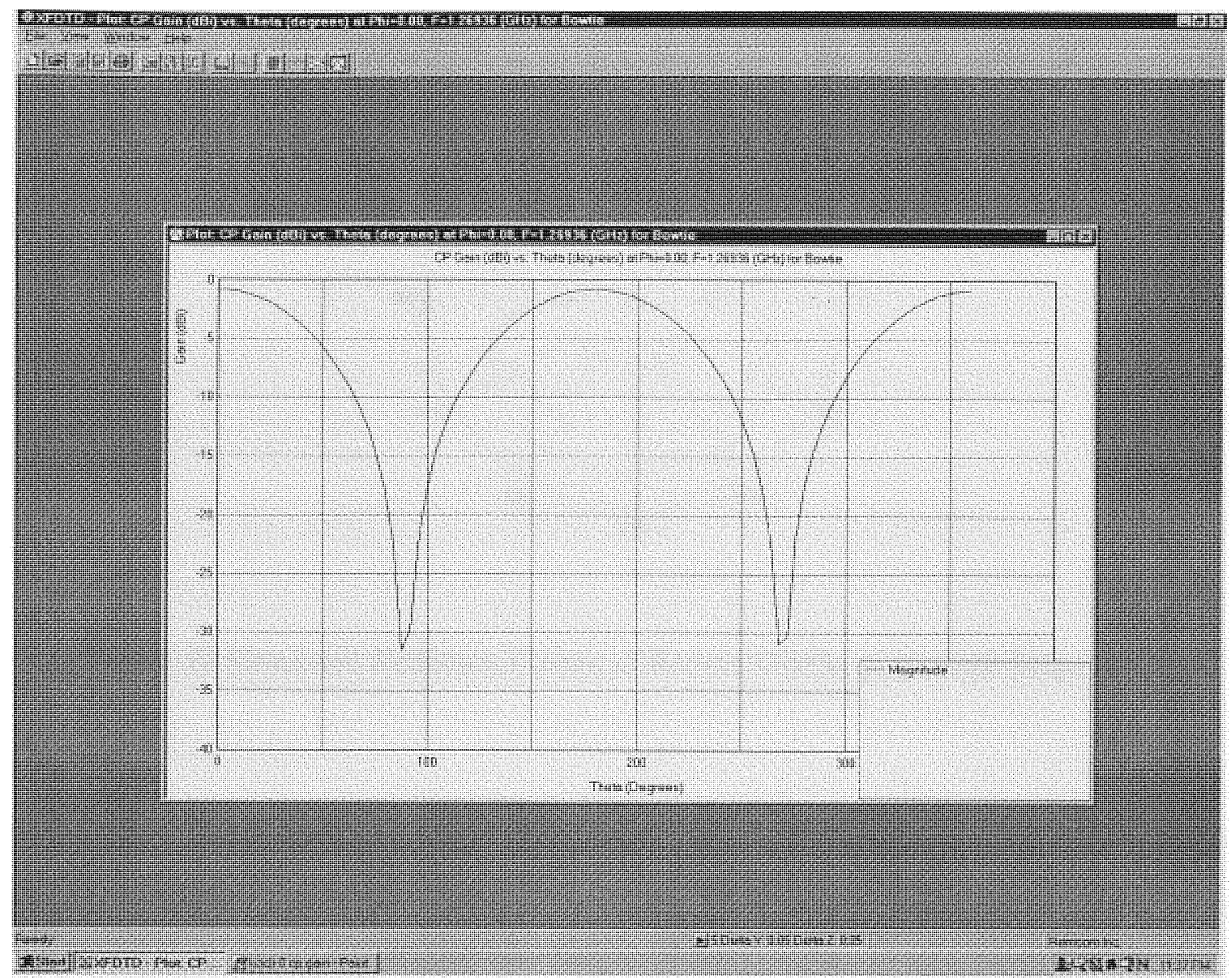

Figure 18 FDTD Simulated Constant Phi Gain

The figure above shows the Gain vs Angle XFDTD simulation at Constant Phi. 


\subsection{Simulated Radiation Pattern of Bowtie Dipole}

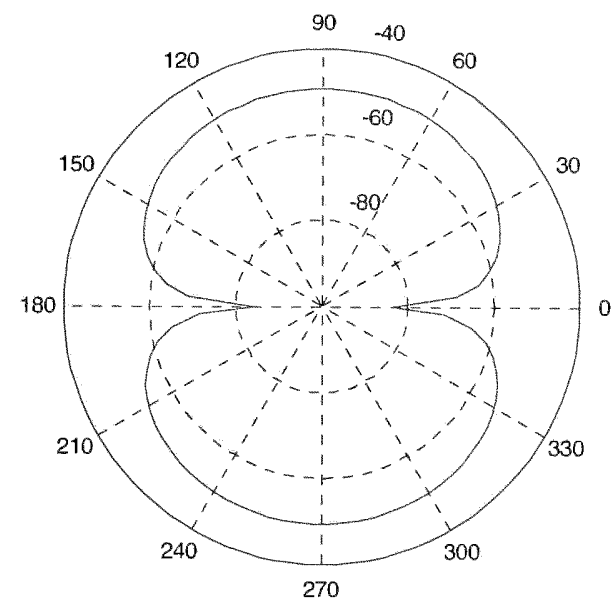

Figure 19 Simulated Radiation Pattern of Bowtie Dipole at resonant frequency.

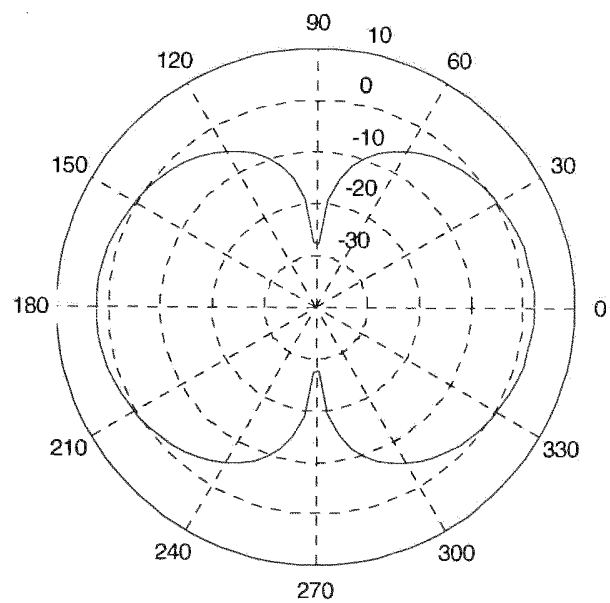

Figure 20 Simulated Radiation Pattern of Bowtie Dipole at resonant frequency. 


\section{Chapter 6 FDTD Analysis of Koch Snowflake Fractal Antenna}

\subsection{Introduction}

In this thesis research, the proposed fractal geometries that are studied are the Koch Snowflake and the Sierpinski Gasket. These structures are implemented to study the properties of fractal theory when applied to antenna design. The main purpose of this research is to show that when fractal theory is applied to antenna design, these fractal antennas can exhibit properties of miniaturizing an antenna and also having broadband and multiband properties.

The fractal structure that enables an antenna to be reduced in size possesses wideband properties and thus maintaining it performance is the Koch Snowflake. The Sierpinski Gasket geometry when applied to an antenna structure give rises to multiband and wideband properties.

Therefore, because of these significant properties when applied to antenna design it has drawn a lot of attention in designing smaller and multiband antenna and thus, drew my interest in researching these fractal antennas structures.

As mention in Chapter 2, the XFDTD numerical platform will be used to study these structures. The results will be compared to conventional antenna with the same design parameters. 


\subsection{Koch Fractal Antenna}

With the increasing demands for developing smaller antennas to meet the advancing in wireless communication devices, leads to the use of fractal geometries in antenna design. As stated in chapter 4, fractal theory when applied to antenna design can reduce the size of an antenna and having wideband properties while retaining its performance. The fractal geometry that allows us to apply the fractal theorem in reducing the size and having wideband properties of an antenna is the Koch Fractals.

Figure19 below shows the development of the Koch Curve.

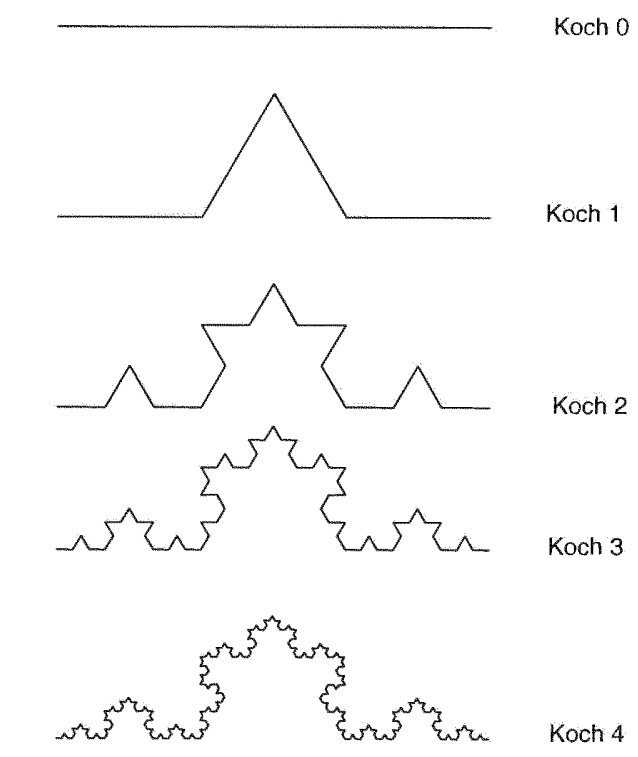

Figure 21 Construction of Koch Curve [9]

The Koch curve consists of four one third sized copies of itself. If we let Koch 0 to be a line segment of unit length. The set Koch 1 consists of four segments obtain by removing the middle third of Koch 0 and replacing it by the other two of the equilateral 
triangle based on the removed segment. Koch 2 is designed by applying the same procedure to each of the segments in Koch 1 and so on. This procedure is applied repeatedly to the remaining lines. Each fourth of the structure is a rescaled copy of the entire structure.

\subsection{Koch Snowflake}

The Koch snowflake fractal starts out with a solid equilateral triangle in the plane. The Koch snowflake is constructed by adding smaller and smaller triangles to the structure in an iterative manner. The geometry is developed by replacing each of the sides of an equilateral triangle by a Koch curve. The three Koch curves are joined together to form the Koch snowflake structure. The Koch snowflake has been primarily used to develop new designs for miniaturizing antennas.

This Koch snowflake structure has been studied in [16] and it was shown that at fundamental mode the Koch snowflake fractal antenna has a resonant frequency that is lower than that of its conventional Euclidean shaped antennas. The resonant frequency and the quality factor are of interest in the study of the behavior of the Koch snowflake antenna. The behavior of the quality factor Q with each Koch snowflake fractal iterations, are observed in [18]. 
The quality factor $\mathrm{Q}$, as related to $1 / B W$, for UWB antennas is given by McLean [18] as:

$$
Q=\frac{1+2(k r)^{2}}{(k r)^{3}\left(1+(k r)^{2}\right)}
$$

Where the wavelength in $k=2 / \lambda$ is the geometric mean of the wavelengths at the band edges.

In this thesis, the properties and characteristics of the Koch snowflake fractal is studied and compared to a Bowtie dipole antenna.

\subsection{Geometry of Koch Snowflake Antenna}

As stated above the Koch Snowflake fractal is designed and analyzed using the Finite Difference Time Domain (FDTD) method. The first four iterations are analyzed numerically to in order to study the antenna input impedance, bandwidth, return loss and the radiation patterns.

The geometry of the Koch Snowflake fractal is based of the conventional Bowtie dipole antenna structure. The Bowtie dipole antenna as described in Chapter 5 is made up of two equal triangle patches with sides of length $0.225 \lambda$, which is mounted of the FR-4 substrate with a relative dielectric constant $\varepsilon_{\mathrm{r}}=4.7$. The thickness of the substrate is $0.01 \lambda$.

The antenna was modeled using the FDTD model. The 3 dimensional FDTD space was determined. The FDTD computational space has $200 \times 180 \times 20$ cells, $\Delta \mathrm{X}=0.05 \mathrm{~mm}, \Delta \mathrm{Y}=0.05 \mathrm{~mm}$ and $\Delta \mathrm{Z}=0.047 \mathrm{~mm}$. 
The geometry of the Koch snowflake fractal antenna is developed by taking the equilateral triangle patches the make up the Bowtie antenna and then scaled down the equilateral triangle patches by a factor of $1 / 3$, and then adding the resulting triangle the to each side of the original patch triangle.

This procedure of reduction by a factor of $1 / 3$ is then applied to the first iteration and thus, the second iteration of the patch is then scaled down by a factor of $1 / 9$ and the added to the sides of the previous triangles that were formed.

For the iteration procedure the following equations defines the scaling factor $s$ of the triangles used and also the equation of the straight line segments $L$ produced for each iteration.

$$
\begin{gathered}
s=\frac{1}{3^{n+1}} \quad \text { for } n=0,1,2, \ldots \ldots \ldots . . \\
L=3 \cdot 4^{n} \quad \text { for } n=0,1,2, \ldots \ldots \ldots
\end{gathered}
$$

Since the Koch Snowflake fractal is formed by using the iteration procedure, it should be noted that at different iterations stages the area of the Koch fractal will differ. That is, the area of the structure increases when you perform different iteration stages. The equation below best describes how the area of the next iteration is computed. 
The area formed at the $\mathrm{n}^{\text {th }}$ iteration for each of the patches is calculated by using the following equation:

$$
A_{n+1}=A_{n}+\frac{\sqrt{3}}{12}\left(\frac{4}{9}\right)^{n-1} \cdot a^{2}
$$

Where $a$, as shown in Figure 22 is the length of one side of the original triangle.

Also, it is shown that the area of the original triangle is given by:

$$
A_{0}=\frac{\sqrt{3}}{4} \cdot a^{2}
$$

With increasing iteration, it is seen that the geometric area converges to

$$
A=\frac{2}{5} \sqrt{3} \cdot a^{2}
$$

Where all the iterations are circumscribed inside a circle of radius $r$ given by:

$$
r=\sqrt{3} a / 3
$$

Another property in that shows significant changes is the perimeter of the Koch Snowflake fractal geometry. It is shown by the equation below that the perimeter of the fractal increases at each iteration stages. The total perimeter for iteration $n$ is given as follow:

$$
l_{n}=3 a\left(\frac{4}{3}\right)^{n}
$$


It is seen that from the geometry of the Koch Snowflake fractal, this geometry possesses self-similarity attributes. With this known fact, the Koch geometry has a selfsimilarity dimension $D$ which is shown by the equation below:

$$
D=\frac{\log (n)}{\log \left(\frac{1}{s}\right)}
$$

Where $n$ is the number of copies of the original geometry which is scaled down by a factor of $s$. This equation can be said to show how each different part are scaled down by similarities with the different scale down factor.

The geometry of the Koch Snowflake fractal antenna that is studied in this thesis is shown below. It should be noted that the initiator (Koch 0 ) of the Koch snowflake is formed from the Bowtie antenna structure. The scale down factor is then applied to the antenna for three iteration stages and thus generates the final Koch Snowflake fractal Koch 3. 
The figure below shows the construction of Koch Snowflake Fractal.

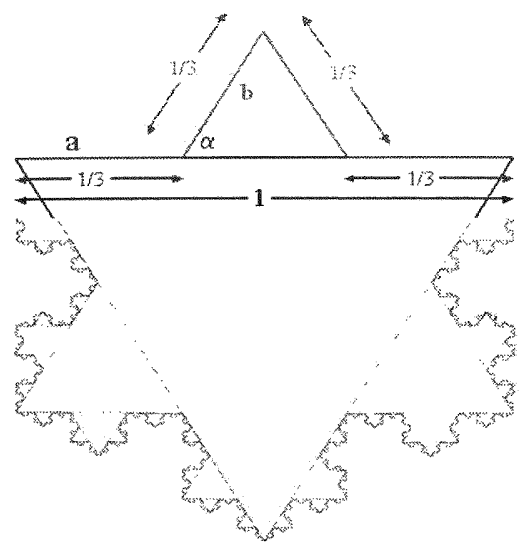

Figure 22 Various Iteration Stages of Koch Snowflake Fractal [9]

Where, $a=1 / 3$ of original side and $b=1 / 3$ of original side of triangle

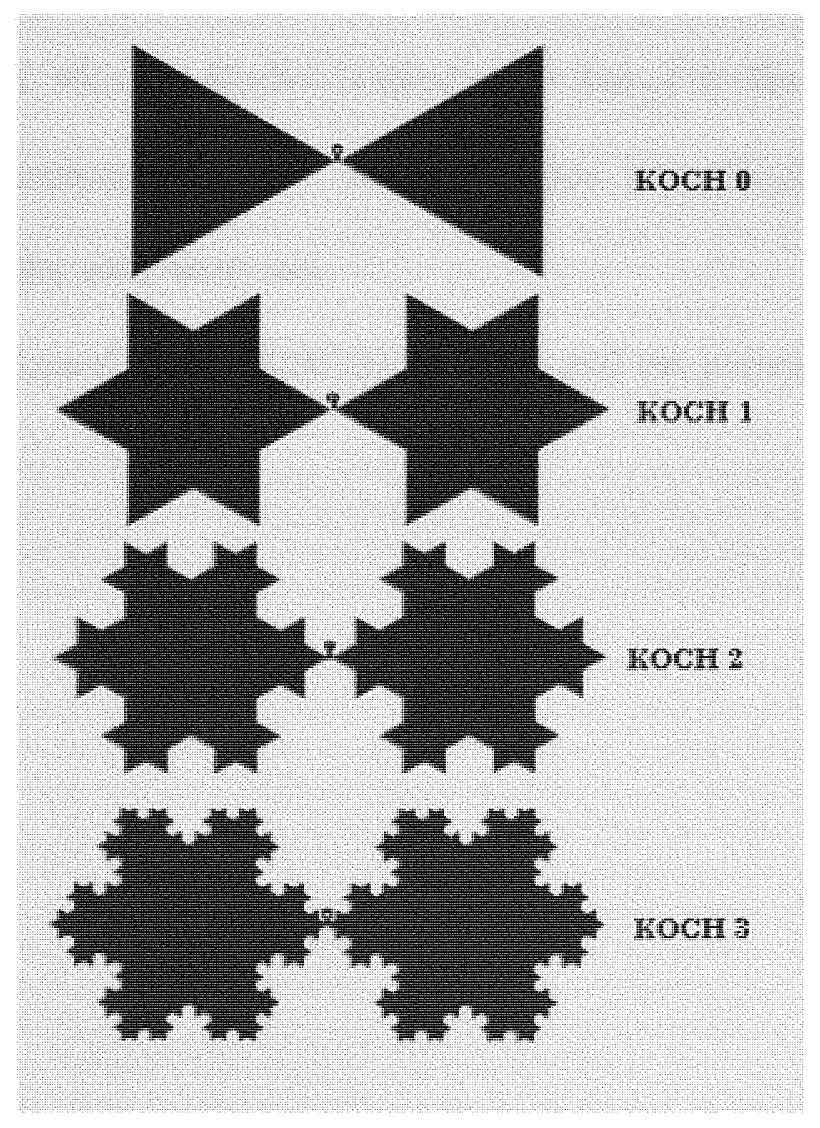

Figure 23 Geometry of Koch Snowflake Fractal Dipole Antenna [9] 
In this study the antenna is implemented on the XFDTD platform. The antenna is fed by using a Modulated Gaussian Pulse of 768 , with the input impedance of $50 \Omega$. The antenna is simulated by using the FDTD numerical method to determine the resonant frequency, the antenna bandwidth and the impedance of the fractal geometries.

\subsection{Results}

From the XFDTD simulation the results of the Koch Snowflake fractal dipole antenna is shown below. The results of the iteration stages will be presented here.

The initiator geometry, which is Koch 0 is identical to that of the Bowtie Dipole antenna. The other iterated geometries where then developed accordingly. They were then simulated and the results were analyzed.

The geometry is implanted on the XFDTD platform and the parameters were set up as follows: 


\subsection{XFDTD Sources / Loads Setup}

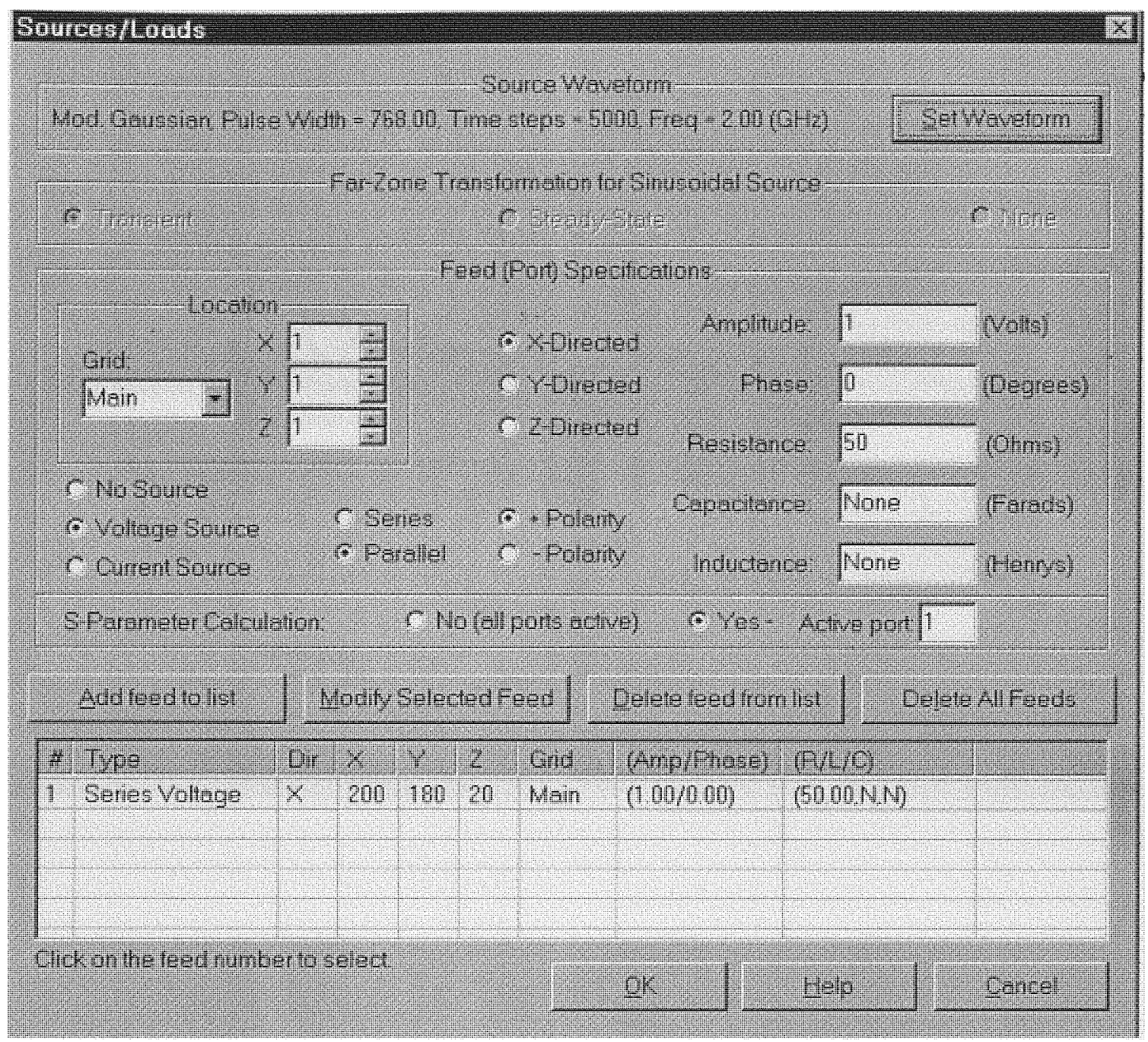

Figure 24 Source / loads Parameters for Koch 0 


\subsection{XFDTD Stimulus Waveform Setup}

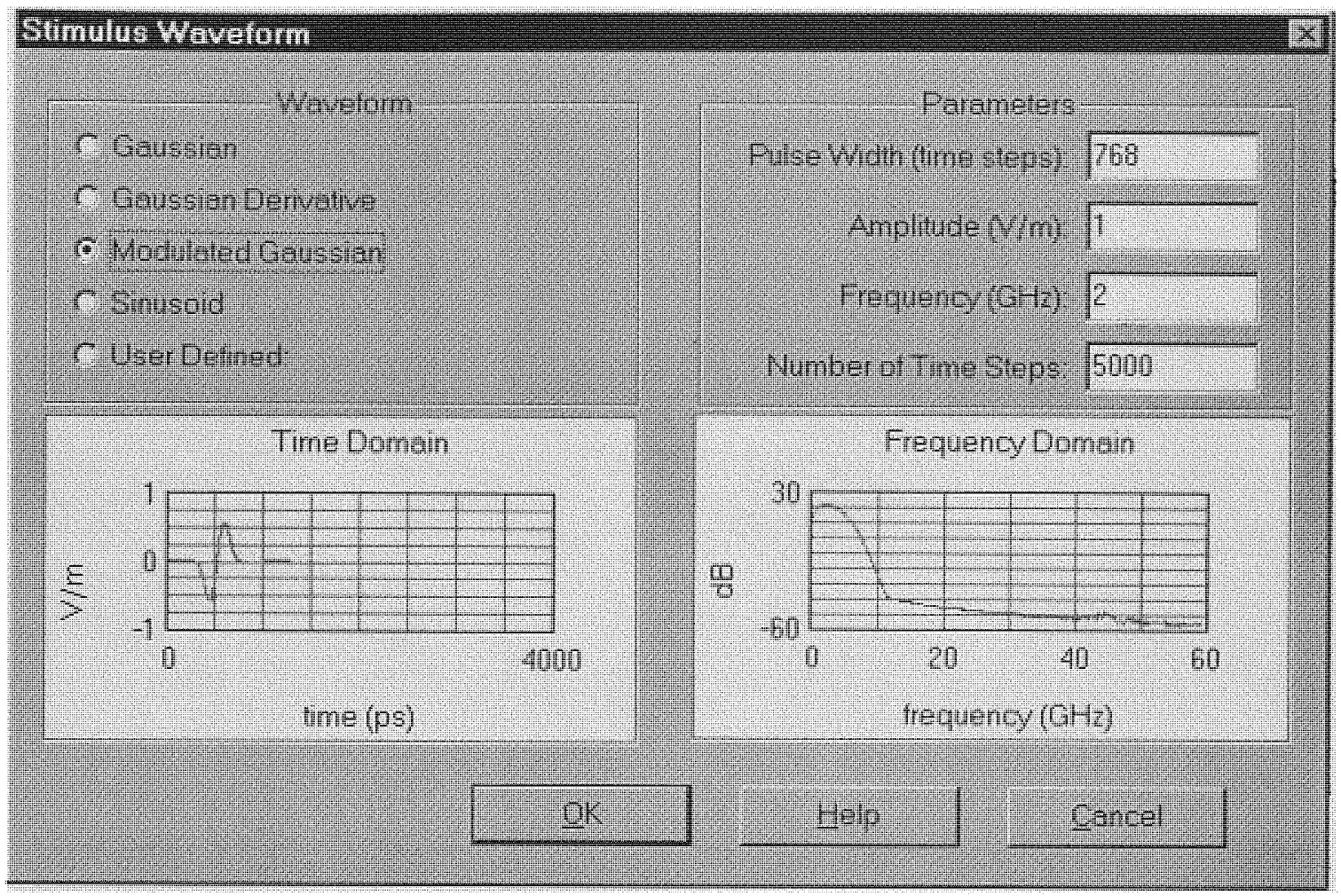

Figure 25 Stimulus Waveform for Koch 0 


\subsection{Koch 0 in XFDTD Space}

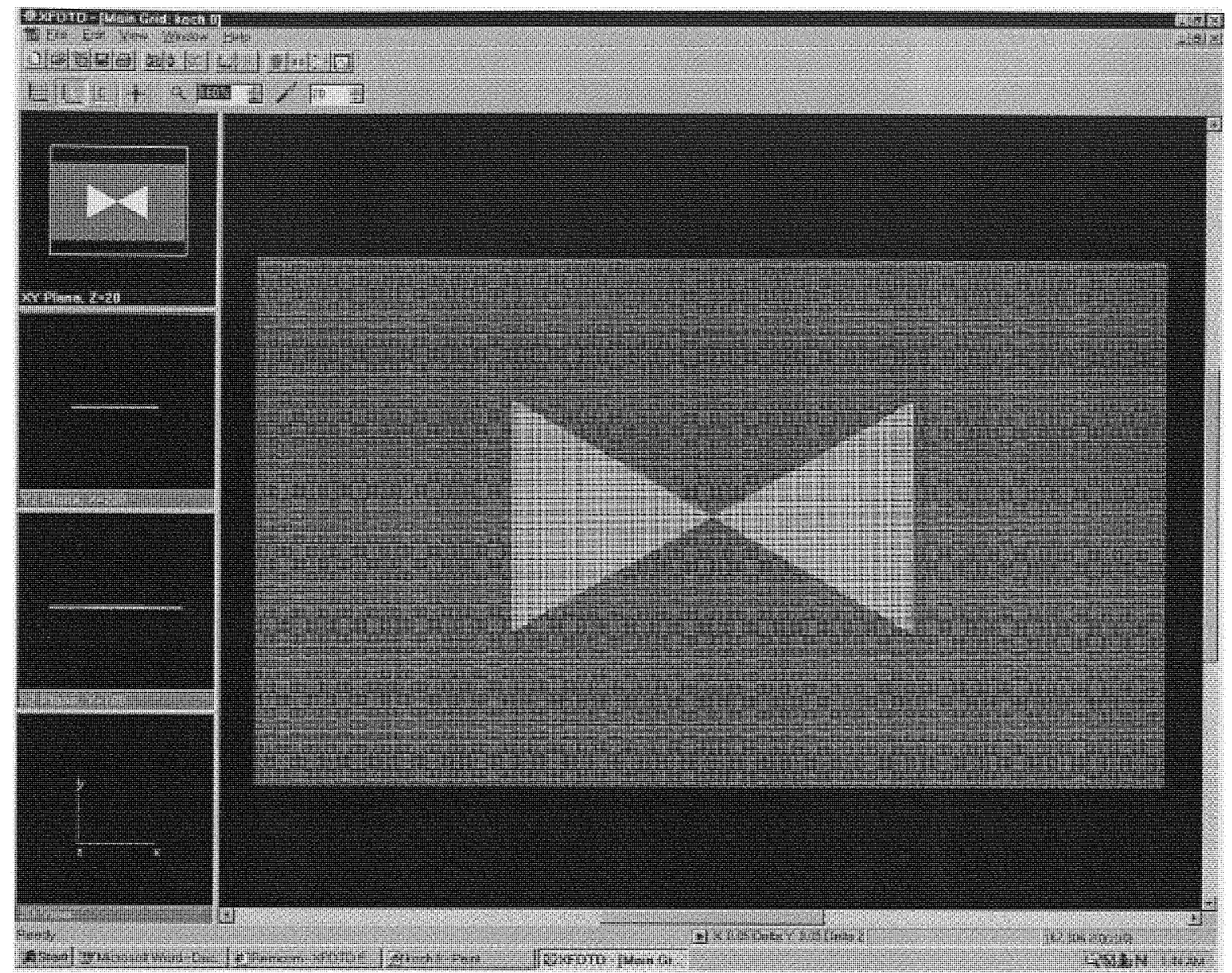

Figure 26 Koch 0 at XY plane in XFDTD space 


\subsection{XFDTD Input Impedance Plot of Koch 0}

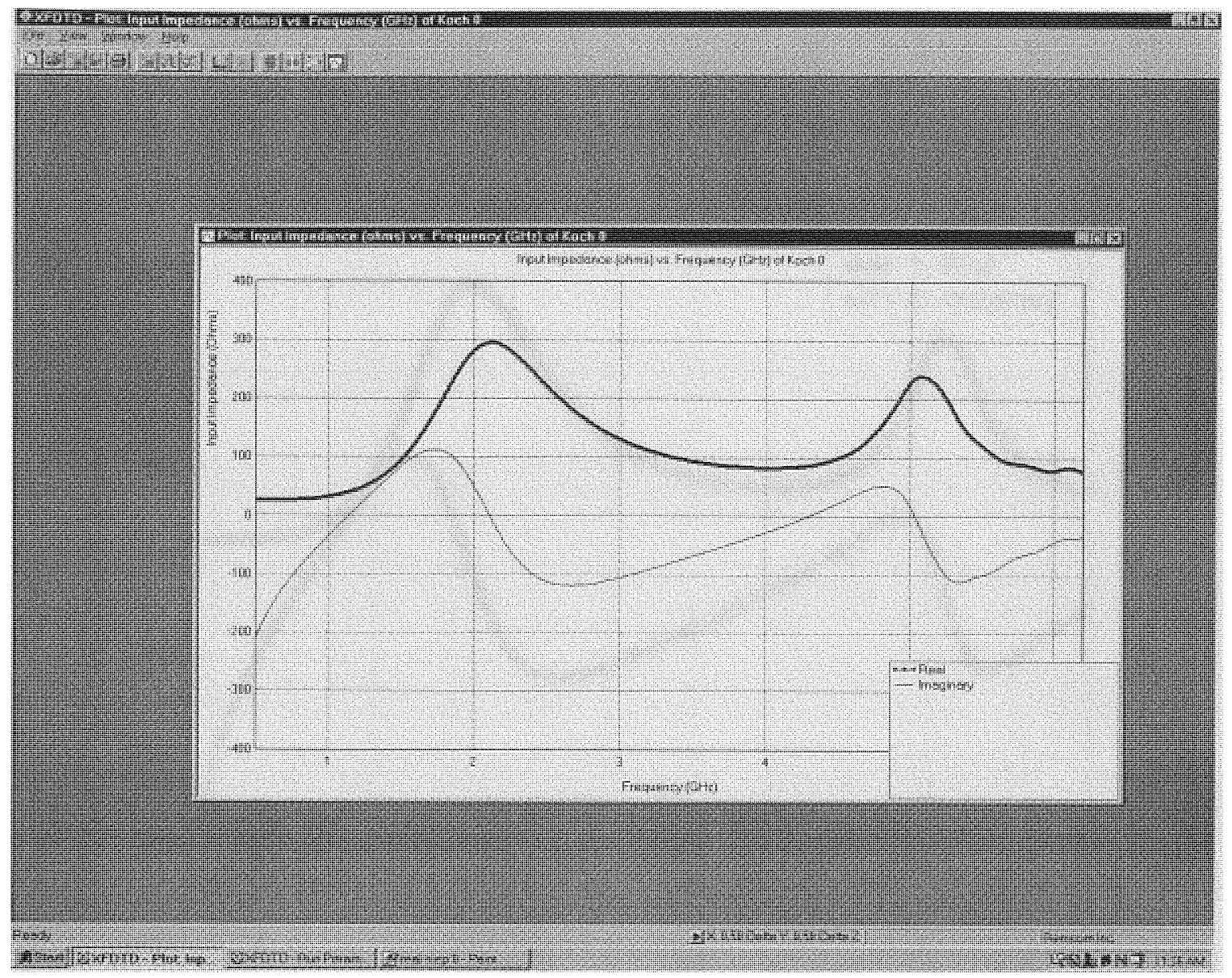

Figure 27 Input Impedance of Koch 0

The figure 27 shows the Imaginary and Real part if the input impedance of Koch 0 fractal. This result was obtained from the XFDTD simulation. 
Figure 28 below shows the Imaginary and Real part if the input impedance of Koch 0 fractal.

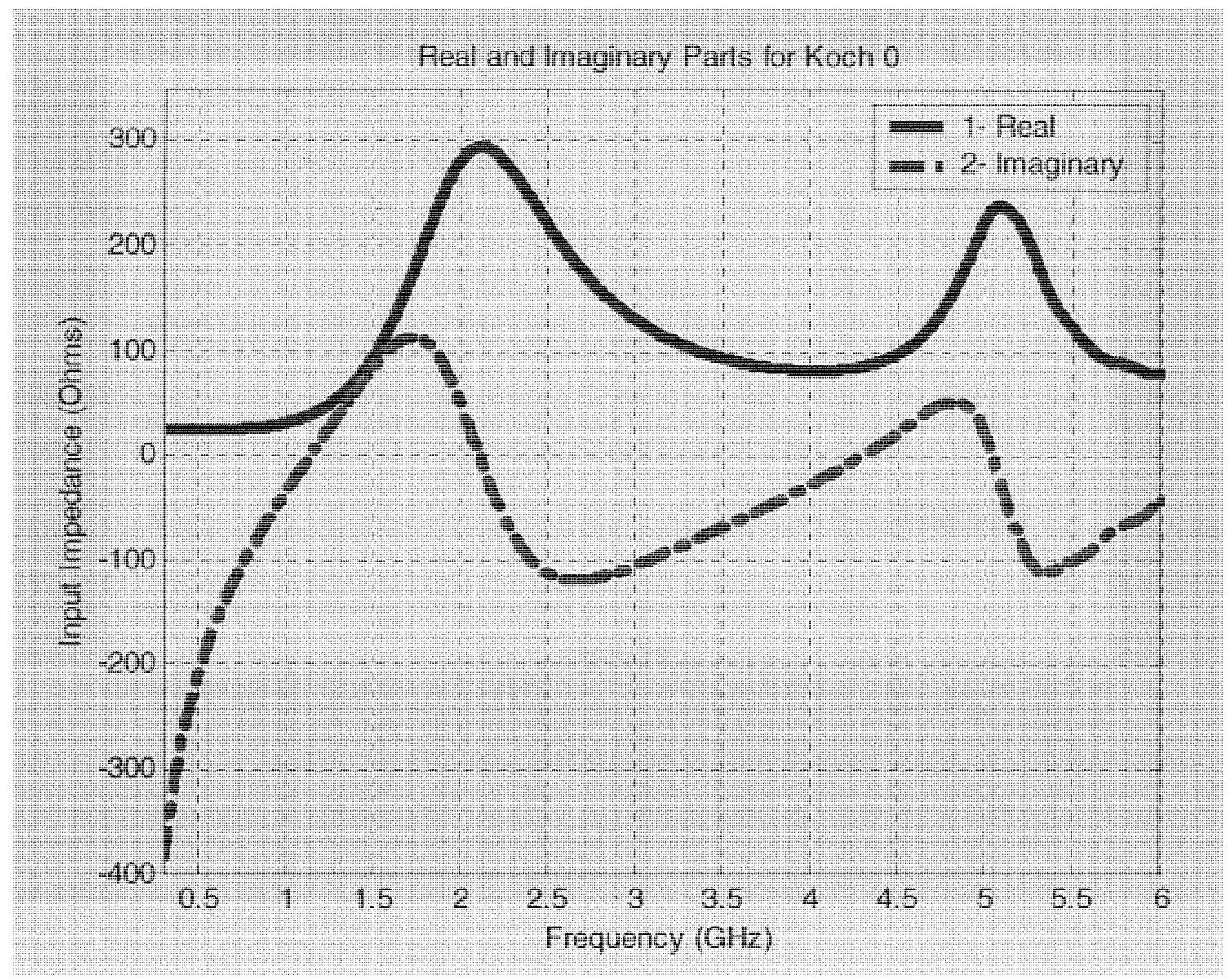

Figure 28 Real and Imaginary Input Impedance of Koch 0

Figure 28 shows the real and imaginary input of Koch 0 . The plot shows that at resonance frequency is about $1.23 \mathrm{GHz}$. 
6.10 FDTD Input Voltage vs Frequency Plot of Koch 0

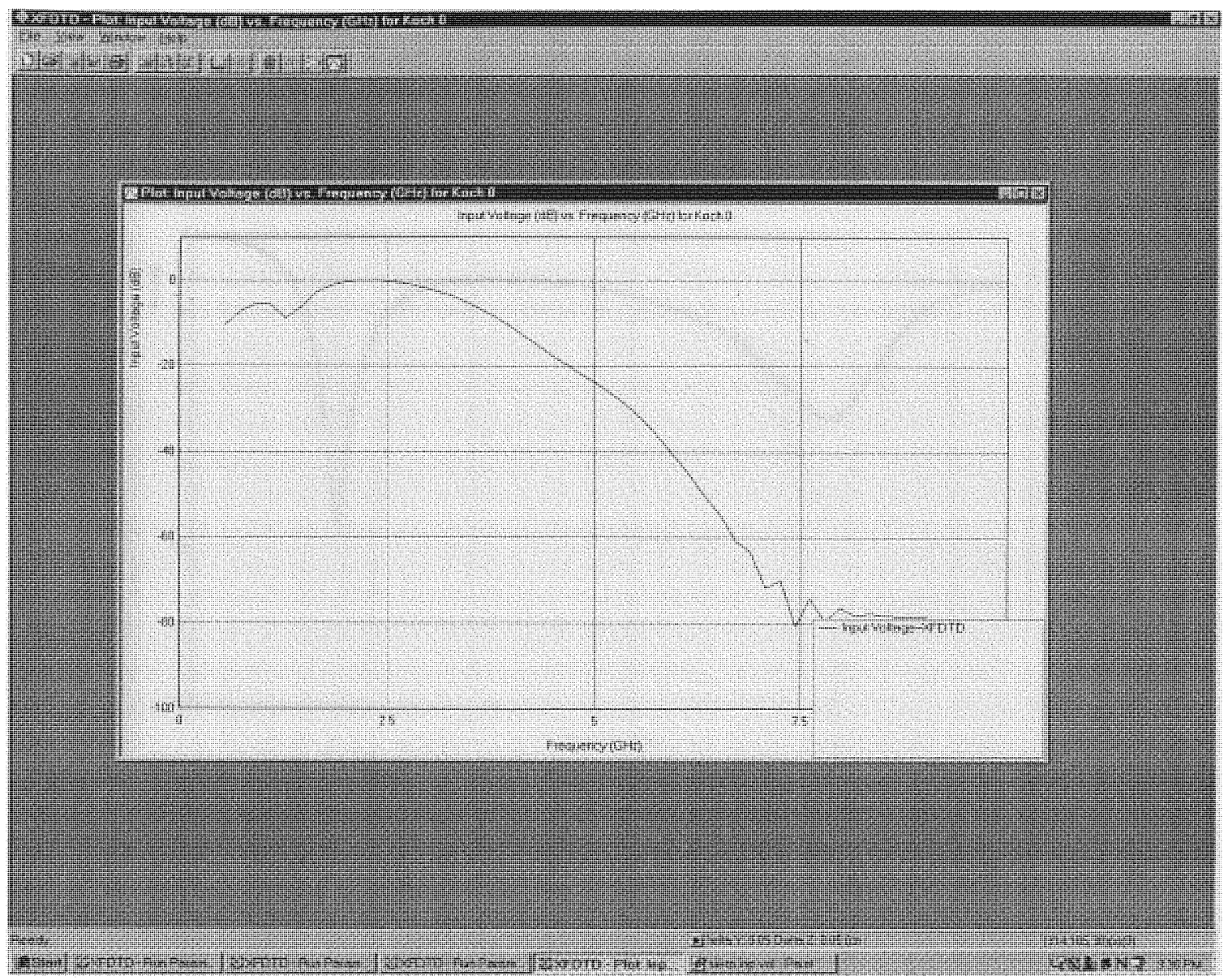

Figure 29 Input Voltage vs Frequency of Koch 0 


\subsection{FDTD $S_{11}$ Parameter Plot of Koch 0}

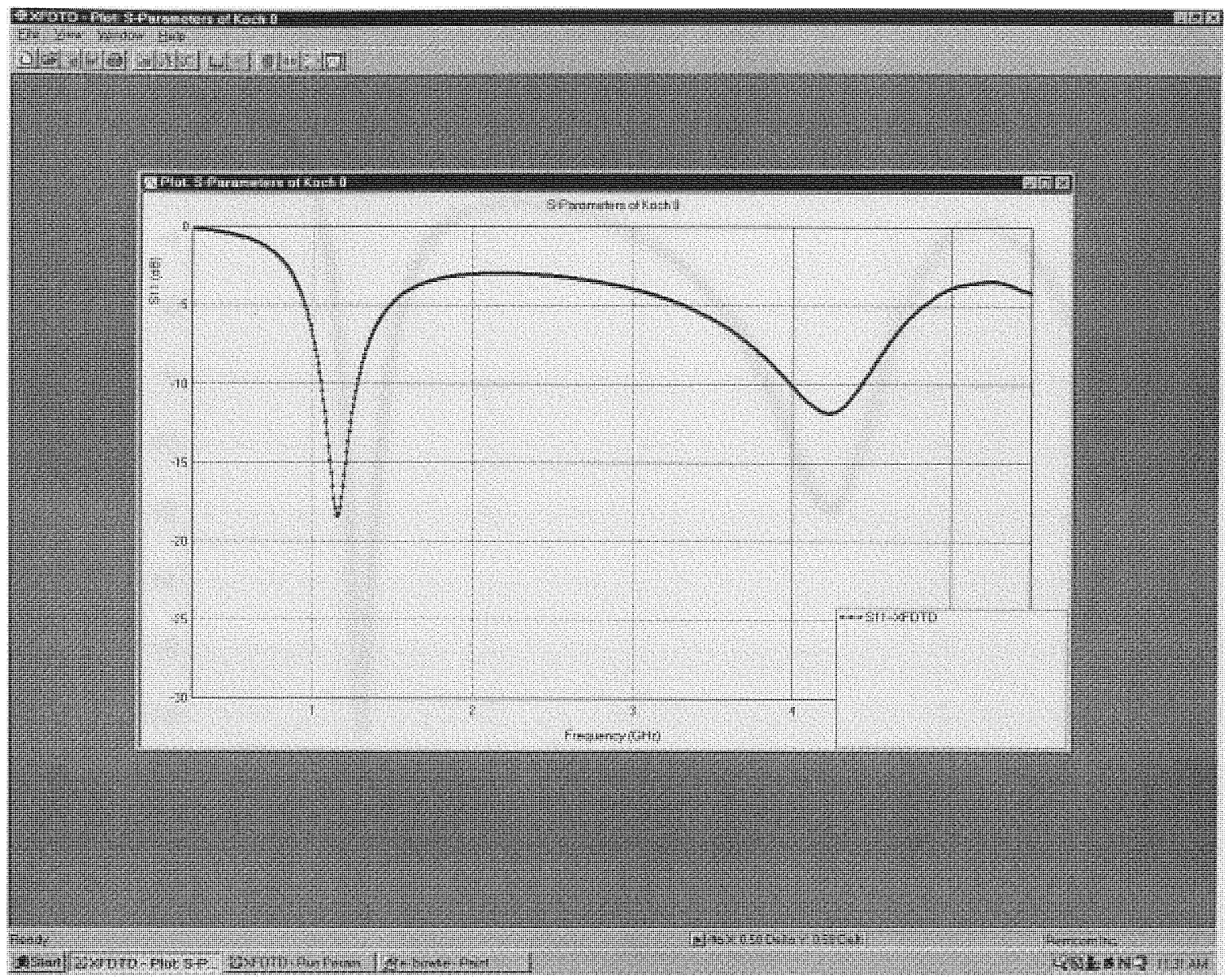

Figure $30 \mathrm{~S}_{11}$ Parameters of Koch 0 


\subsection{Return Loss for Koch 0}

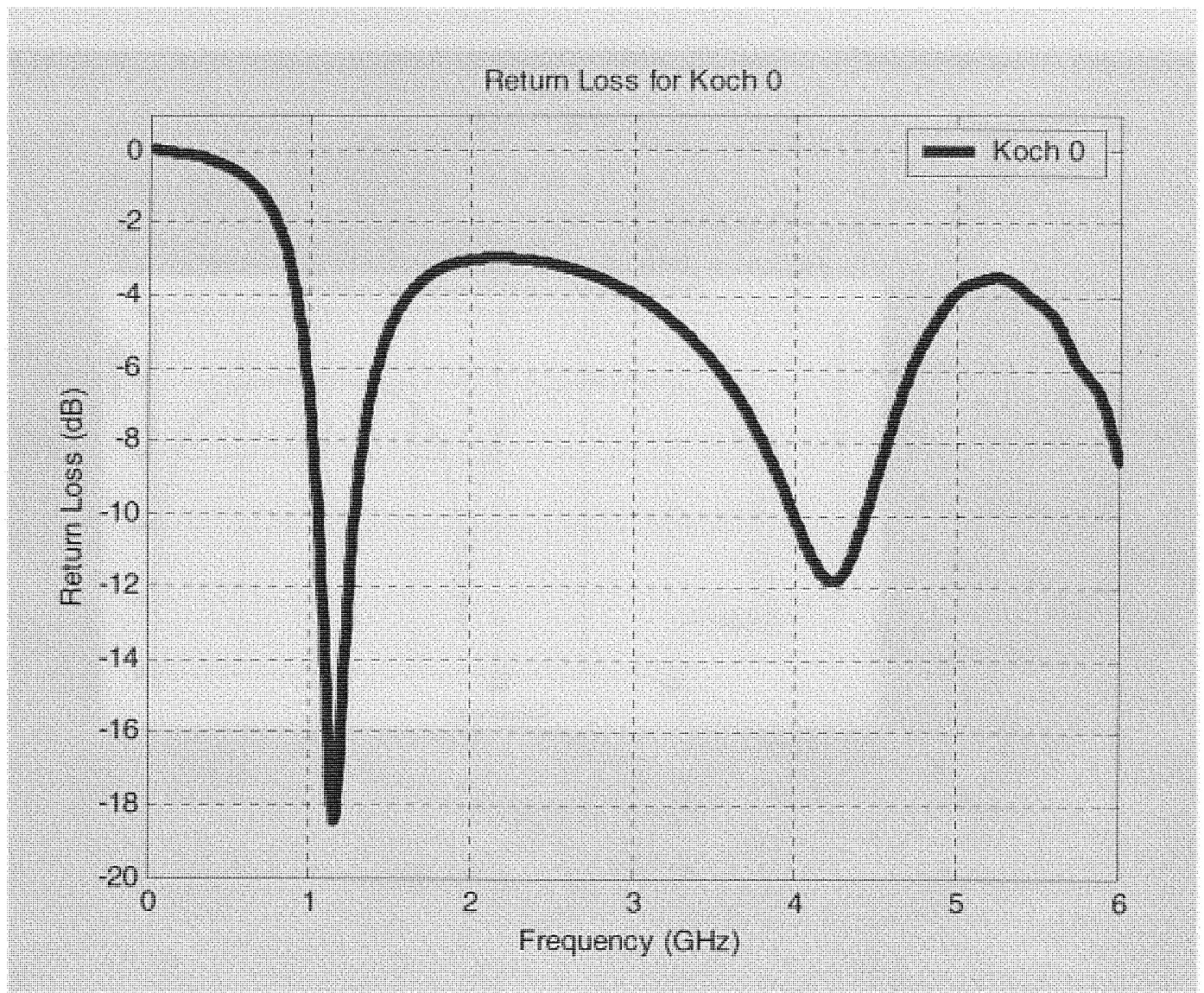

Figure 31 Return Loss for Koch 0

The input impedance bandwidth obtained from the numerical calculation has an operational bandwidth ranges from about $1.23 \mathrm{GHz}$ to $4.3 \mathrm{GHz}$. At resonant frequency of $1.23 \mathrm{GHz}$ it is found that Koch 0 to be $-19 \mathrm{~dB}$ and at $4.3 \mathrm{GHz}$ was $-12 \mathrm{~dB}$. 


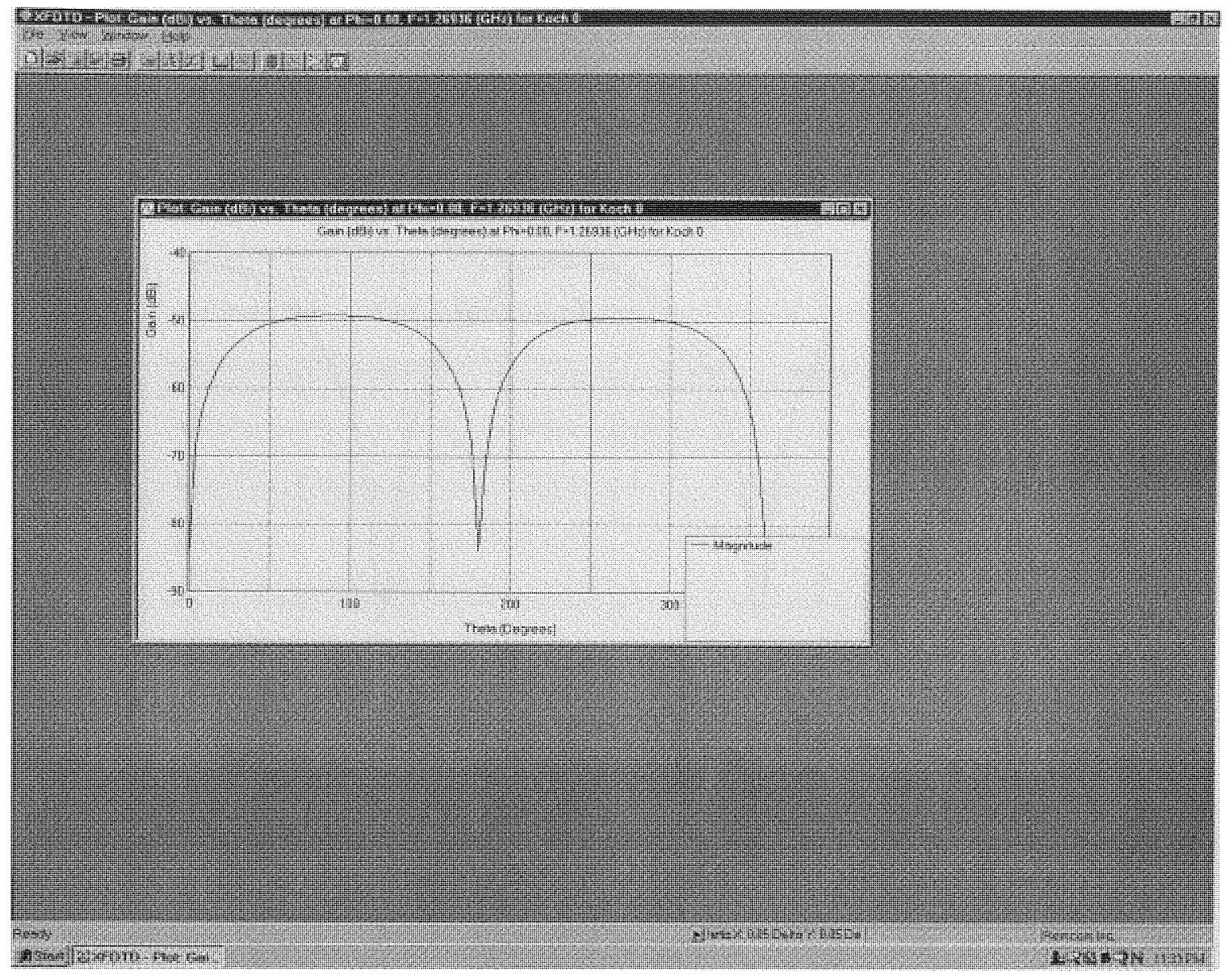

Figure 32 FDTD Simulated Gain of Koch 0

The above figure shows the Gain vs Angle XFDTD simulation. The figure shows the gain vs Theta when Phi is at a constant. 


\subsection{FDTD CP Gain Plot of Koch 0}

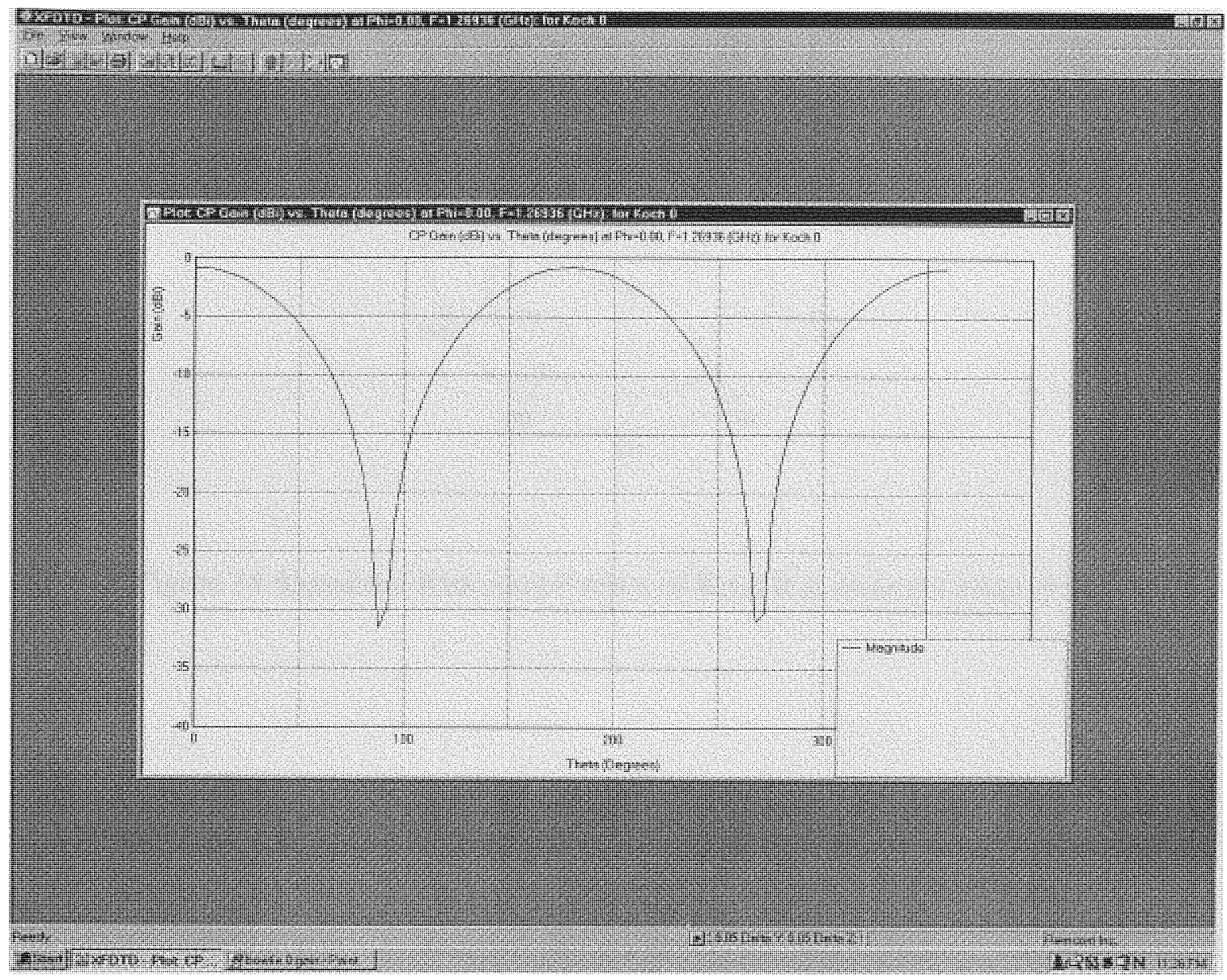

Figure 33 FDTD Simulated Constant Phi Gain of Koch 0

The above figure shows the Gain vs Angle XFDTD simulation at Constant Phi. 


\subsection{Simulated Radiation Pattern of Koch 0}

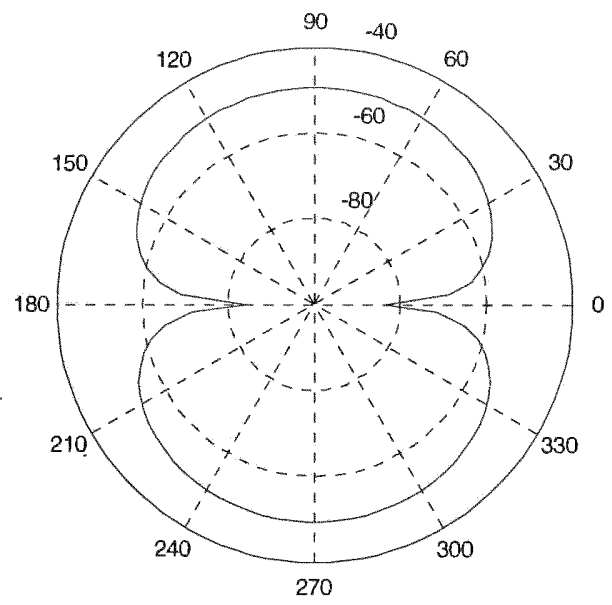

Figure 34 Simulated Radiation Pattern of Koch 0 at resonant frequency.

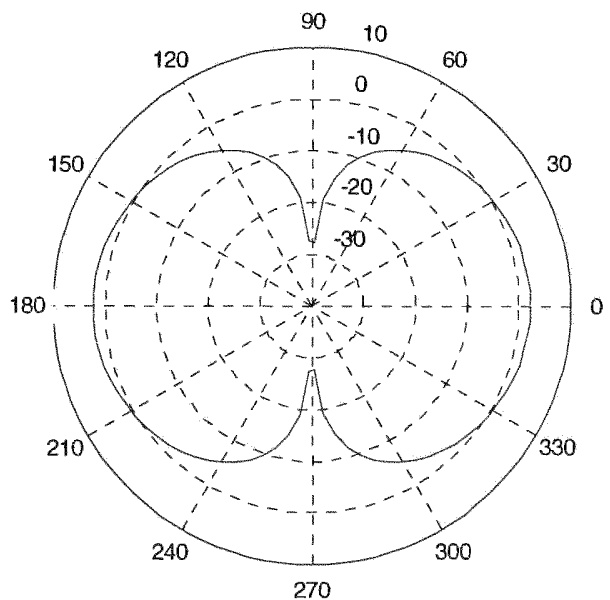

Figure 35 Simulated Radiation of Koch 0 at resonant frequency. 


\subsection{Koch 1 in FDTD Space}

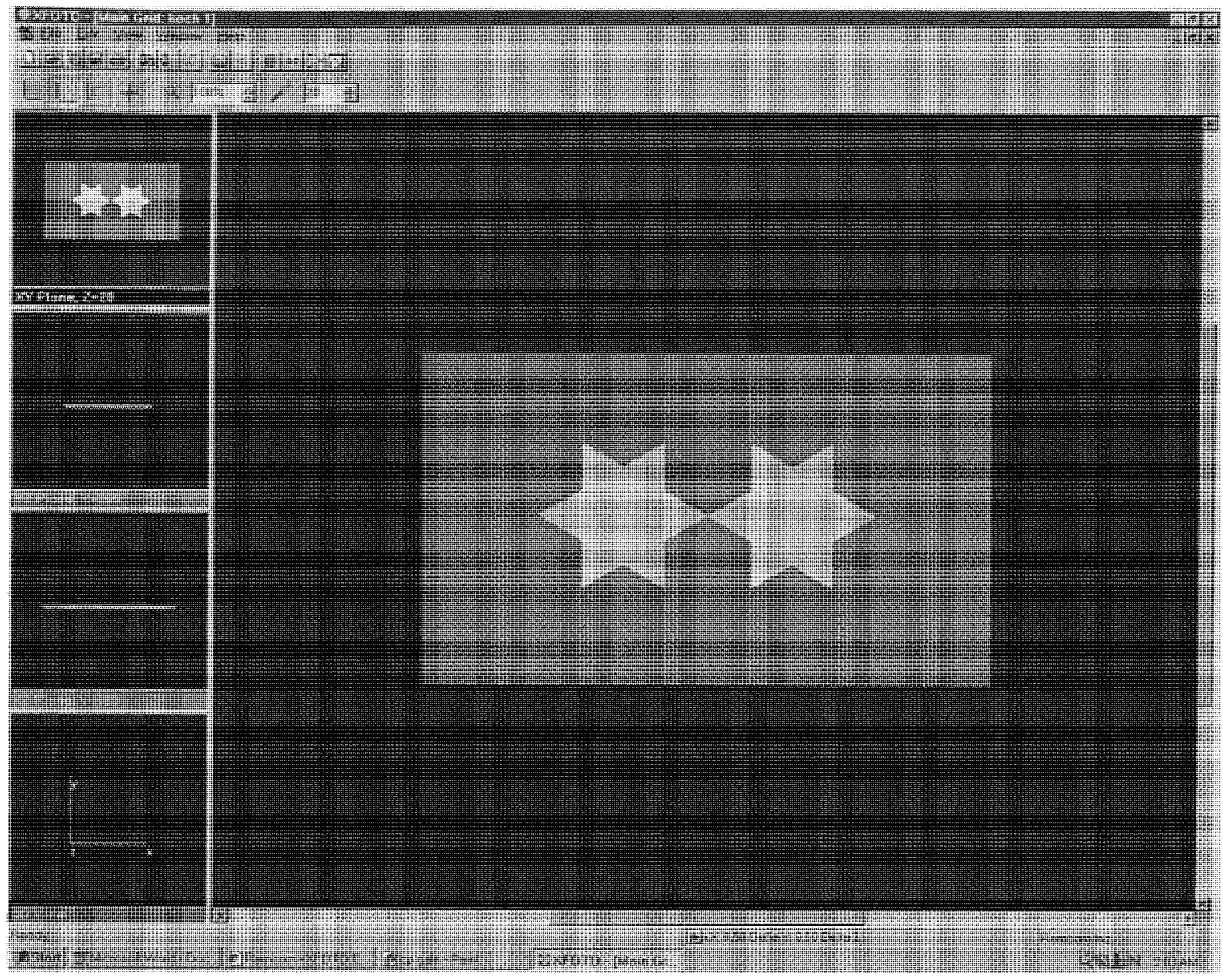

Figure 36 Koch 1 at XY plane in XFDTD space 


\subsection{FDTD Input Impedance Plot for Koch 1}

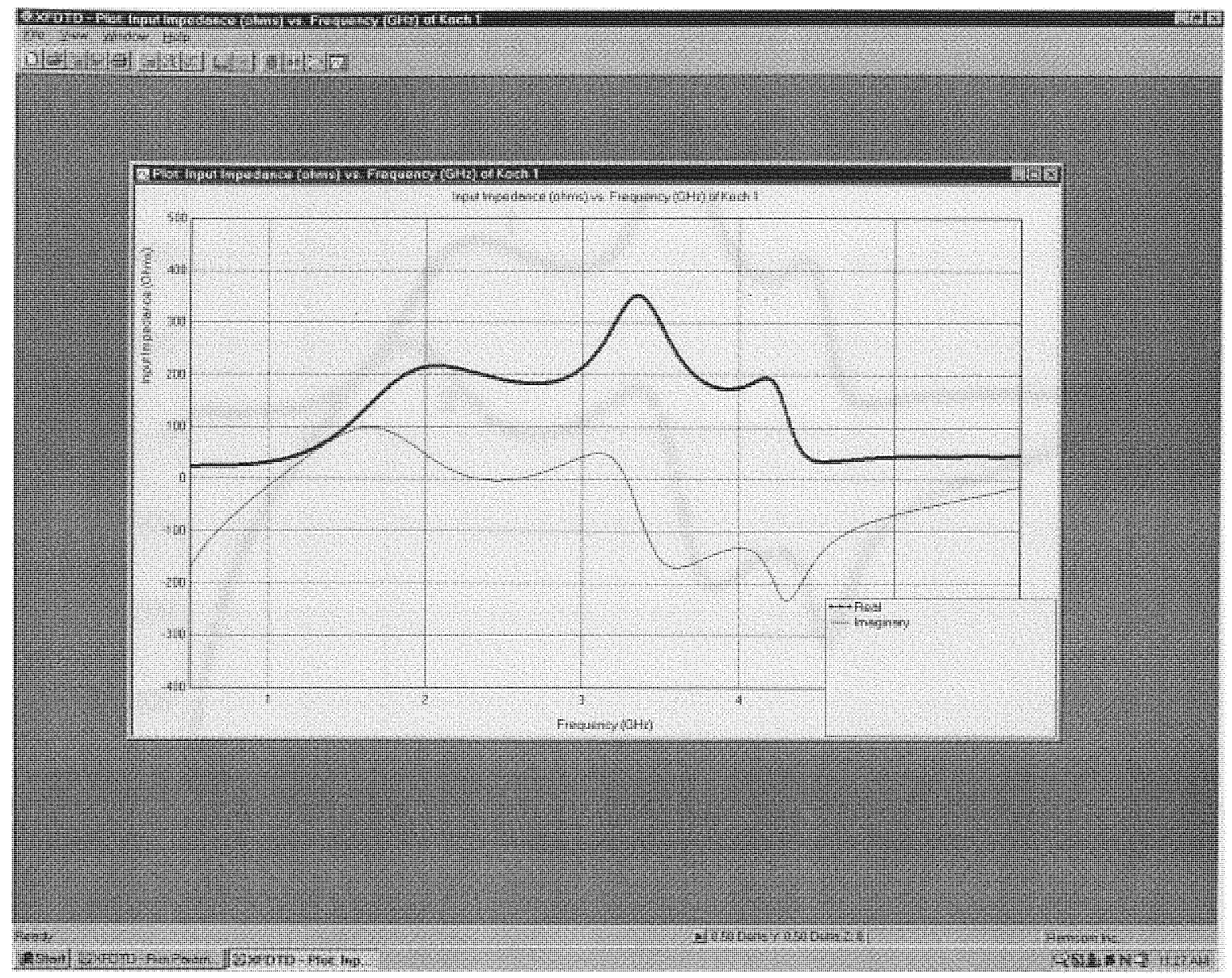

Figure 37 Input Impedance of Koch 1

The above figure shows the Imaginary and Real part of the Koch 1 fractal. The results were obtained from the FDTD simulation. 
Figure 38 below shows the Imaginary and Real part of the Koch 1 fractal

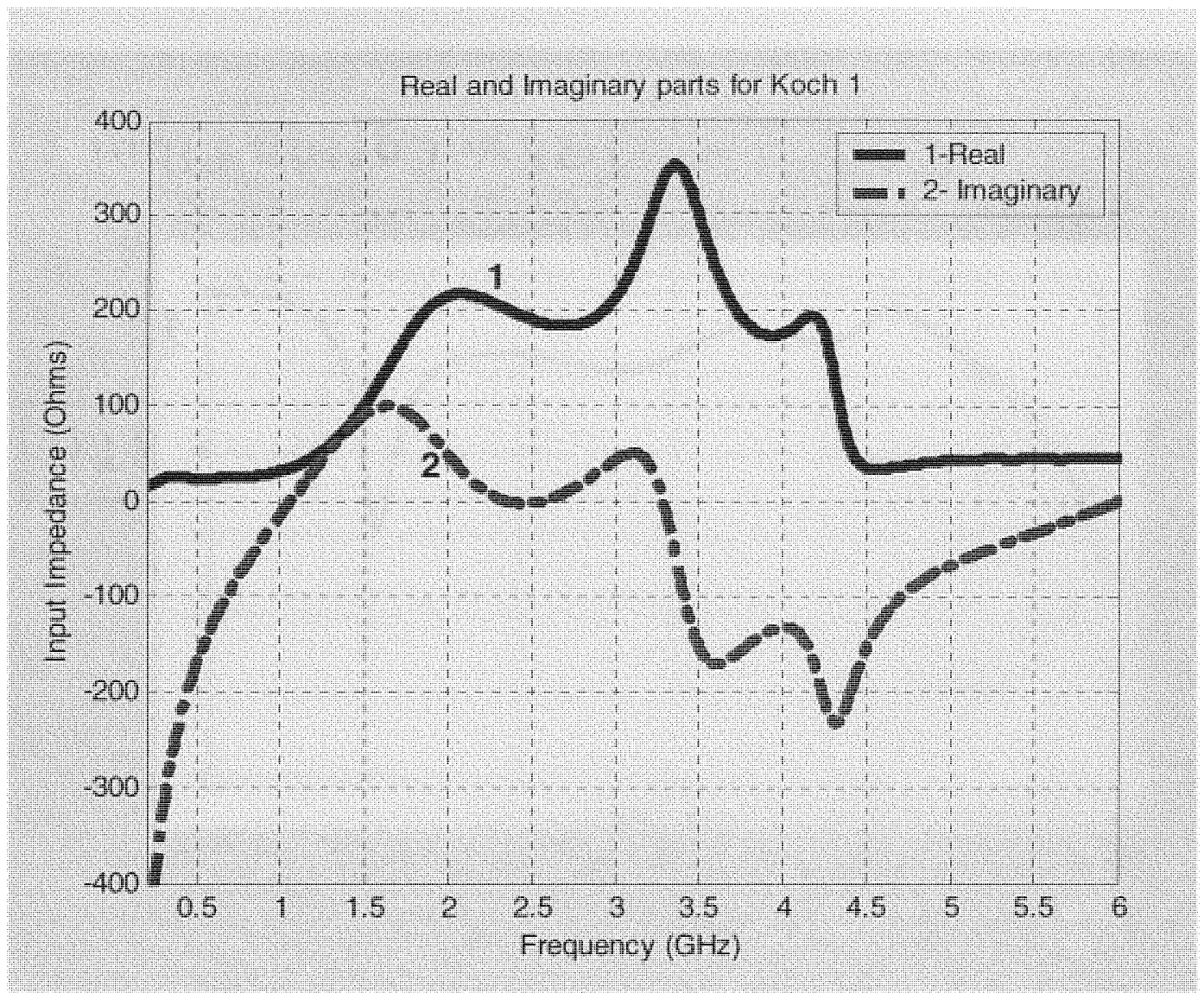

Figure 38 Real and Imaginary Input Impedance of Koch 1 


\subsection{S11 Parameters for Koch 1}

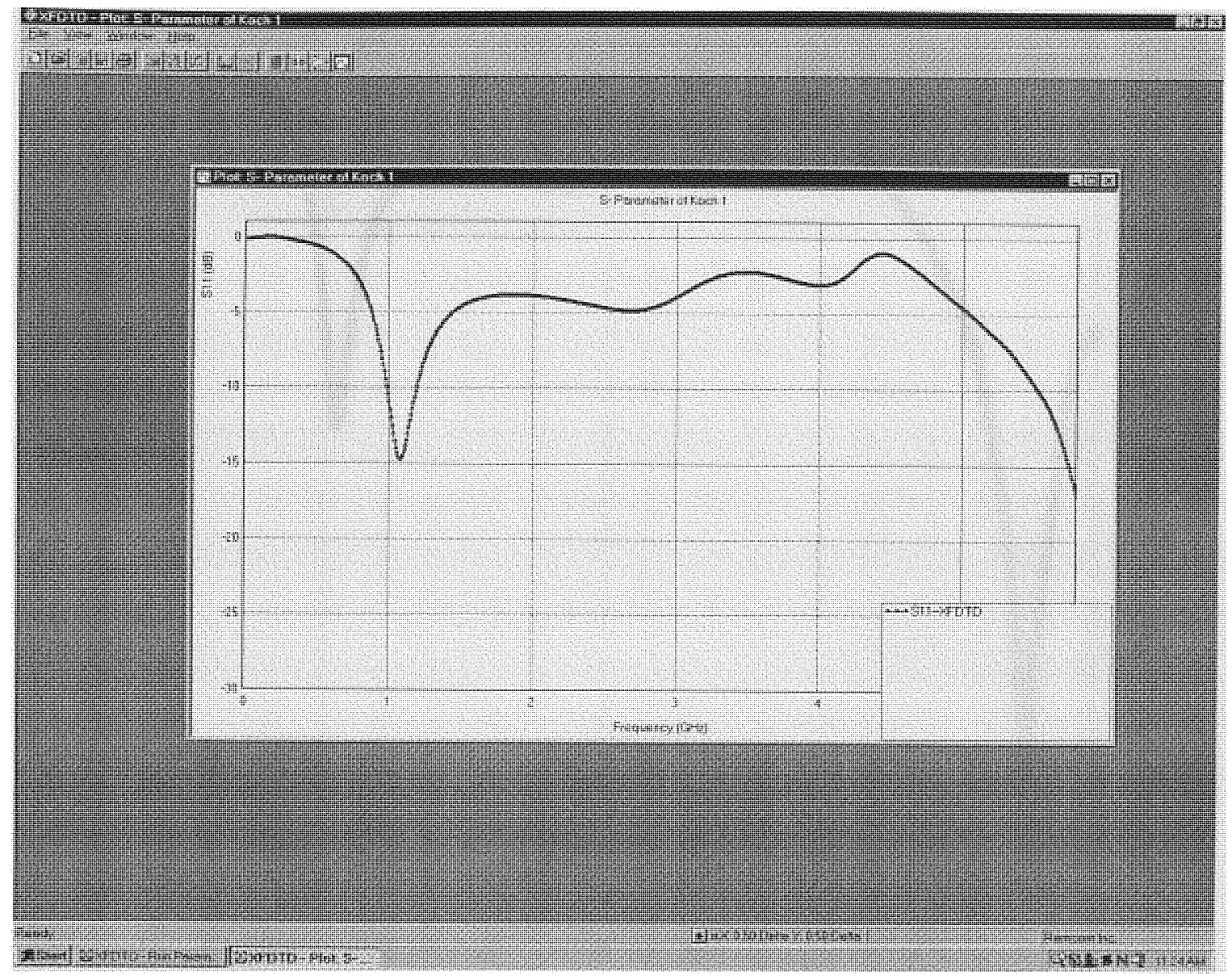

Figure 39 S11 Parameters for Koch 1 


\subsection{Return Loss for Koch 1}

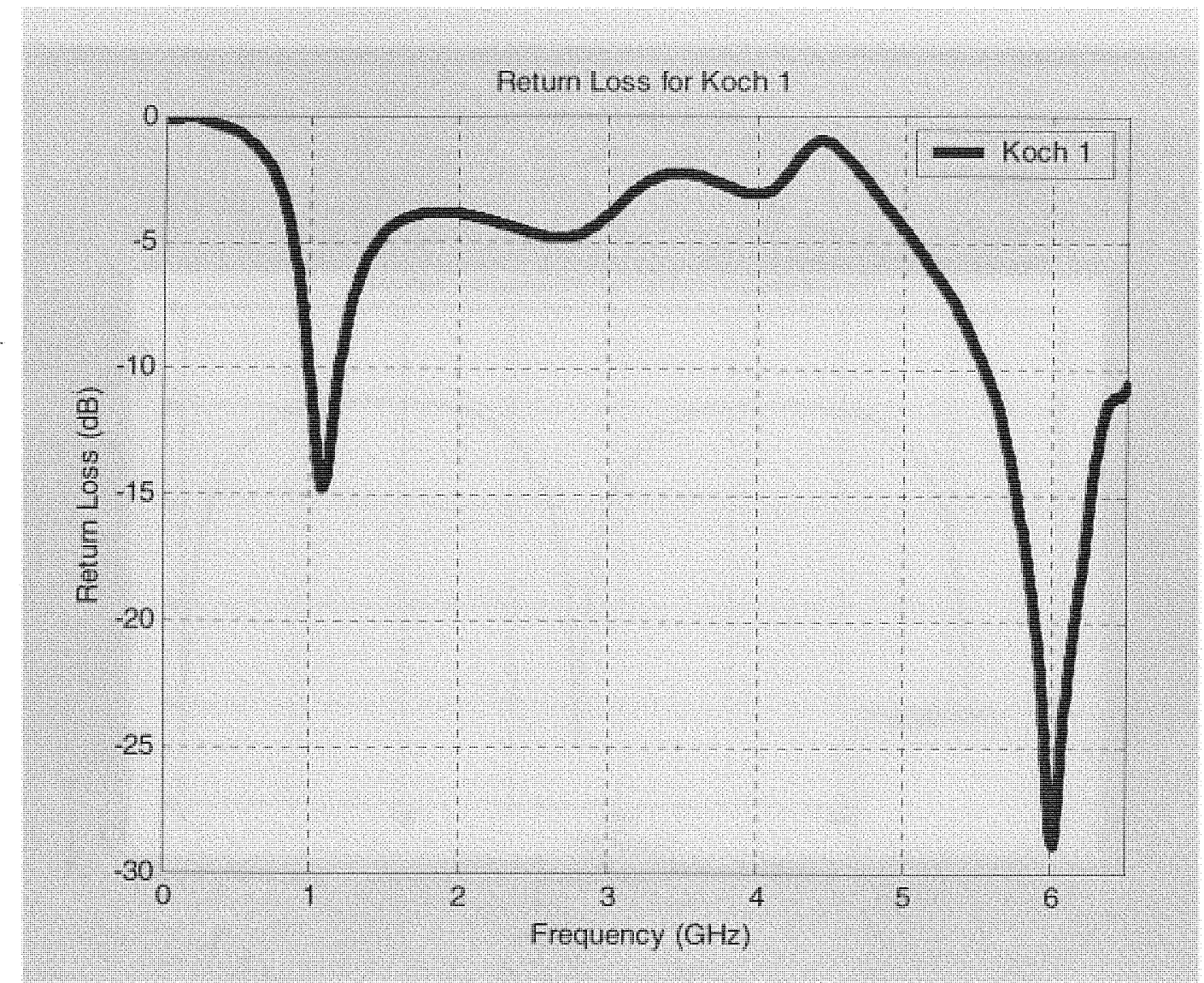

Figure 40 Return Loss for Koch 1

The input impedance bandwidth obtained from the numerical calculation has an operational bandwidth ranges from about $1.22 \mathrm{GHz}$ to $6 \mathrm{GHz}$. At resonant frequency of $1.22 \mathrm{GHz}$ it is found that Koch 1 to be $-15 \mathrm{~dB}$ and at $6 \mathrm{GHz}$ is $-29 \mathrm{~dB}$. 


\subsection{XFDTD Gain Plot for Koch 1}

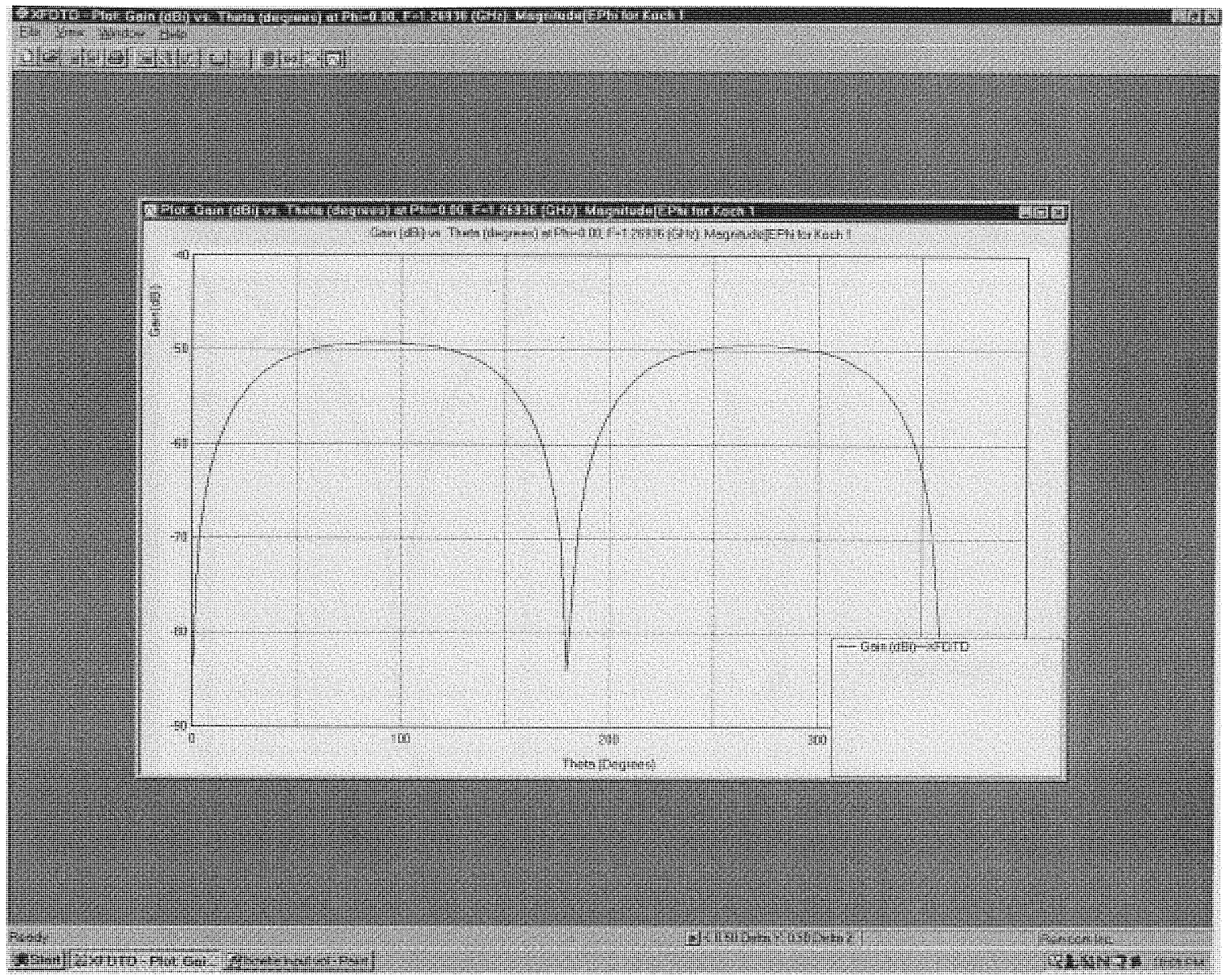

Figure 41 FDTD Simulation Gain Koch 1

The figure above shows the Gain vs Angle XFDTD simulation. 


\subsection{XFDTD CP Gain Plot of Koch 1}

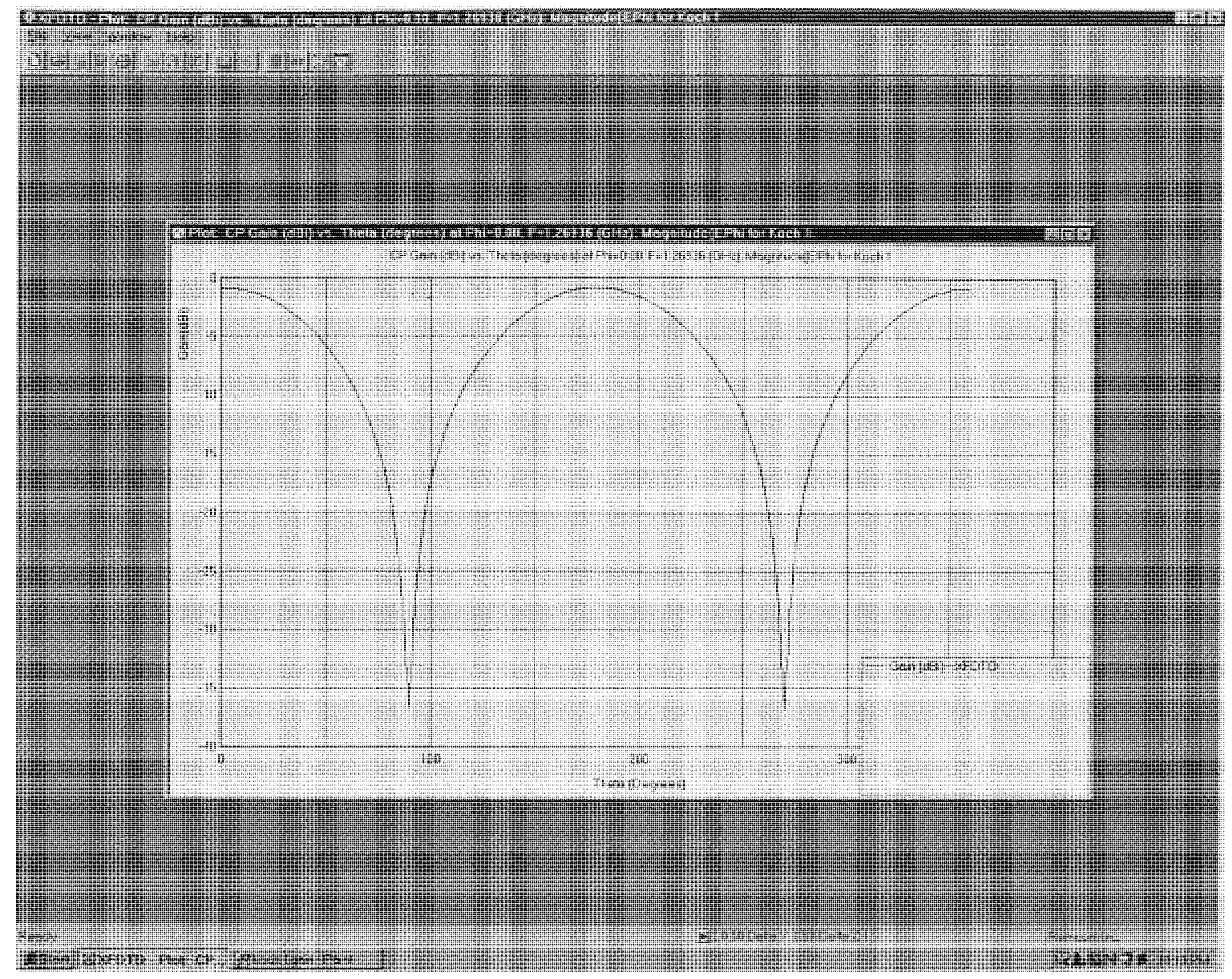

Figure 42 FDTD Simulation of CP Gain vs Theta for Koch 1

The figure above shows the Gain vs Angle XFDTD simulation at Constant Phi. 


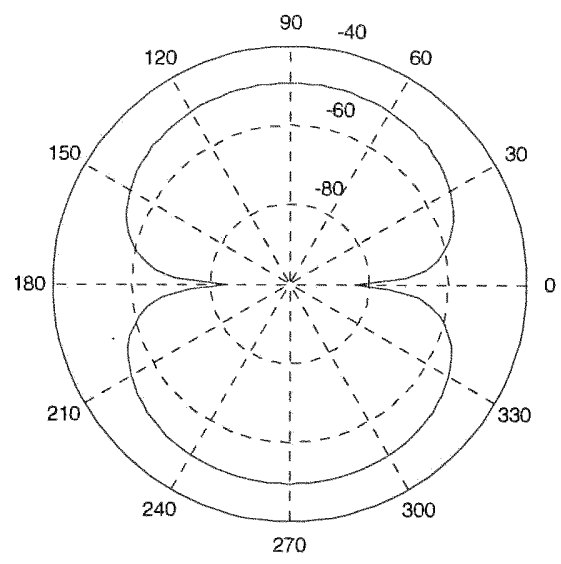

Figure 43 Simulated Radiation Pattern for Koch 1 at resonant frequency.

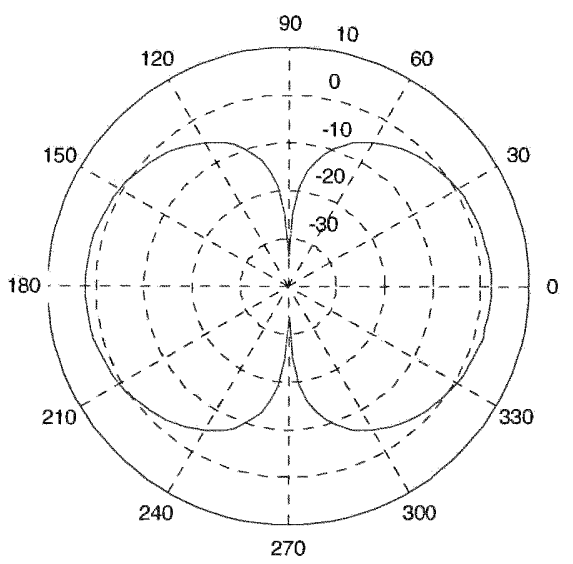

Figure 44 Simulated Radiation Pattern for Koch 1 at resonant frequency. 


\subsection{Koch 2 in XFDTD Space}

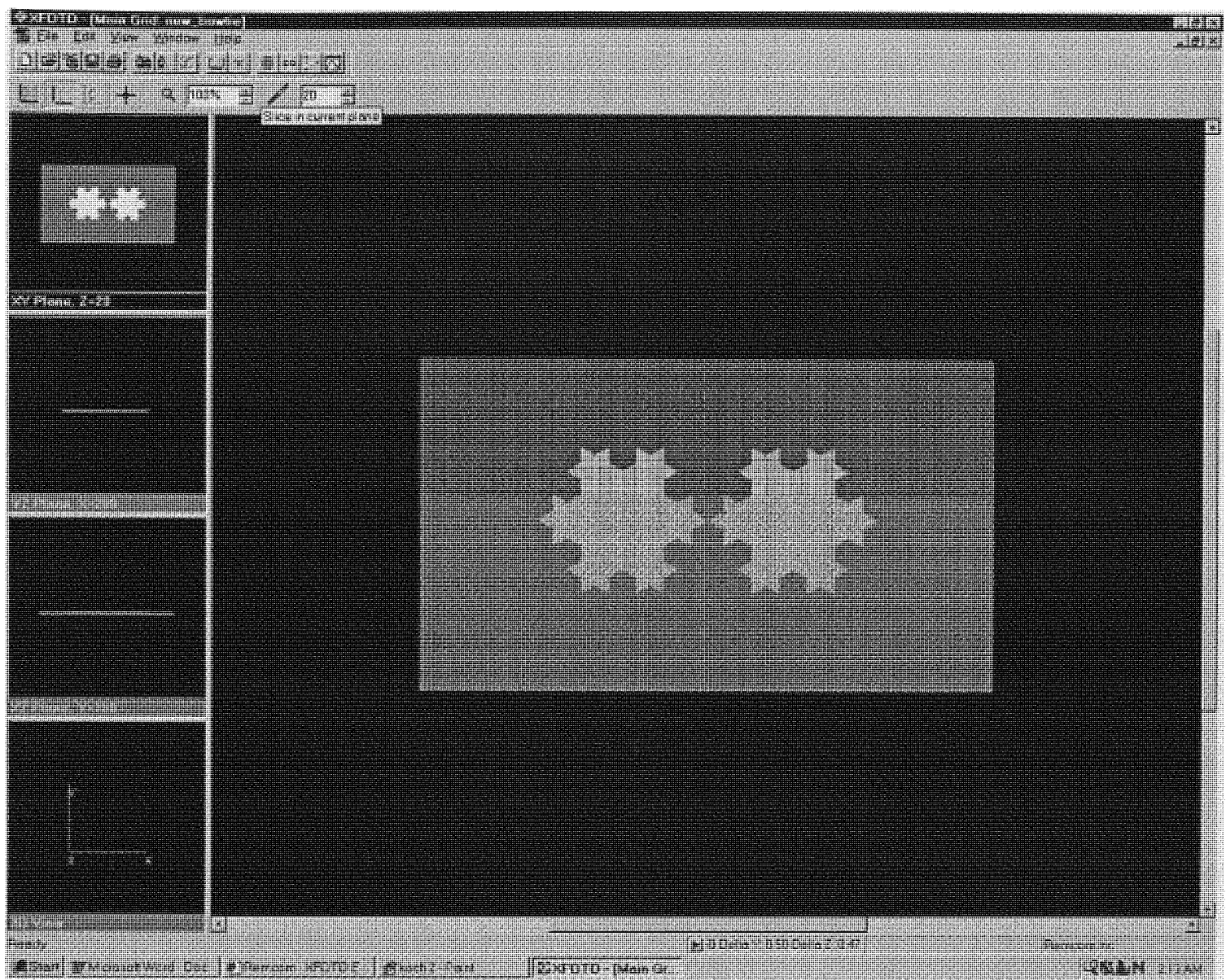

Figure 45 Koch 2 at XY plane in XFDTD space 


\subsection{FDTD Input Voltage vs Frequency Plot of Koch 2}

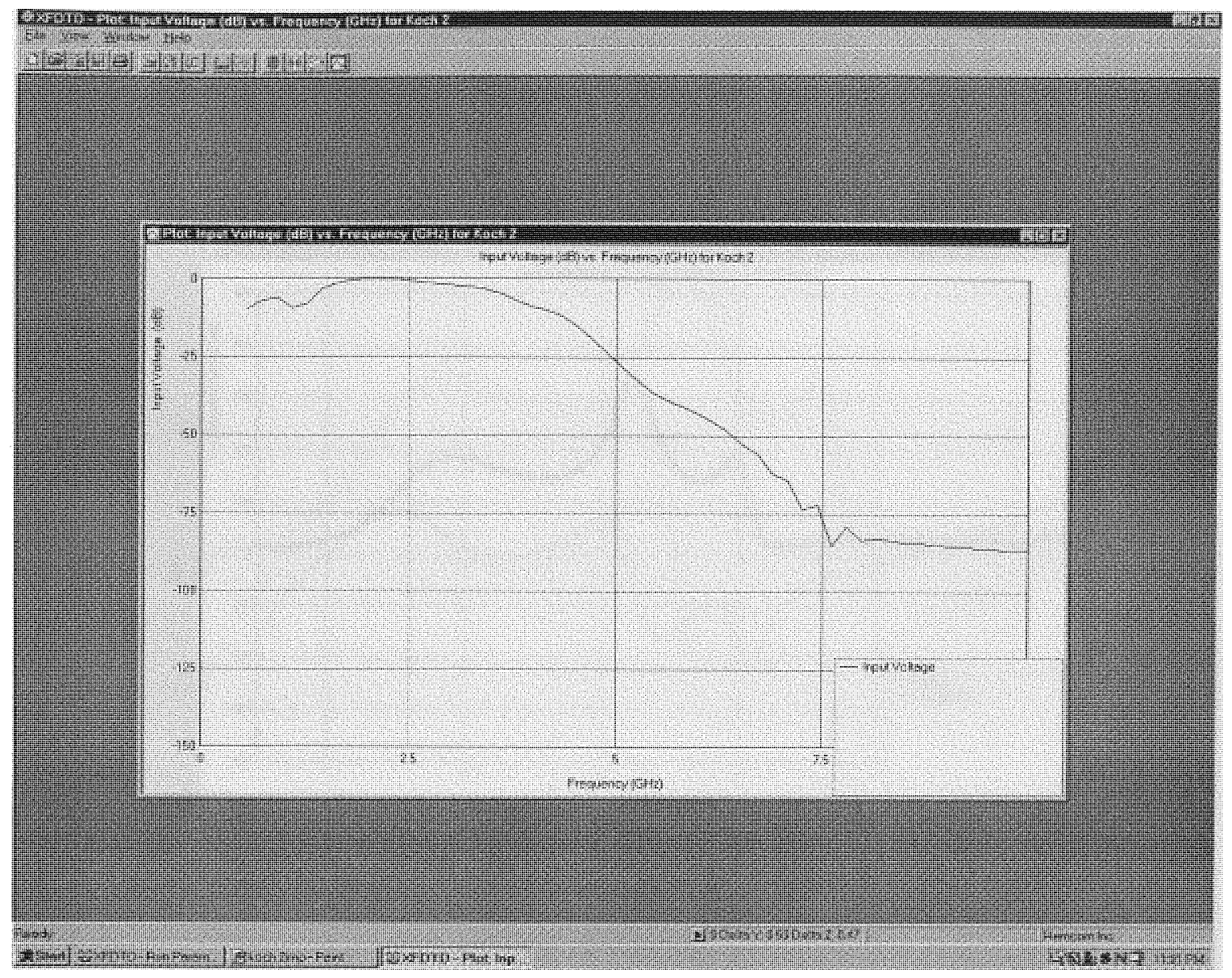

Figure 46 Input Voltage vs Frequency for Koch 2 


\subsection{FDTD Input Impedance Plot for Koch 2}

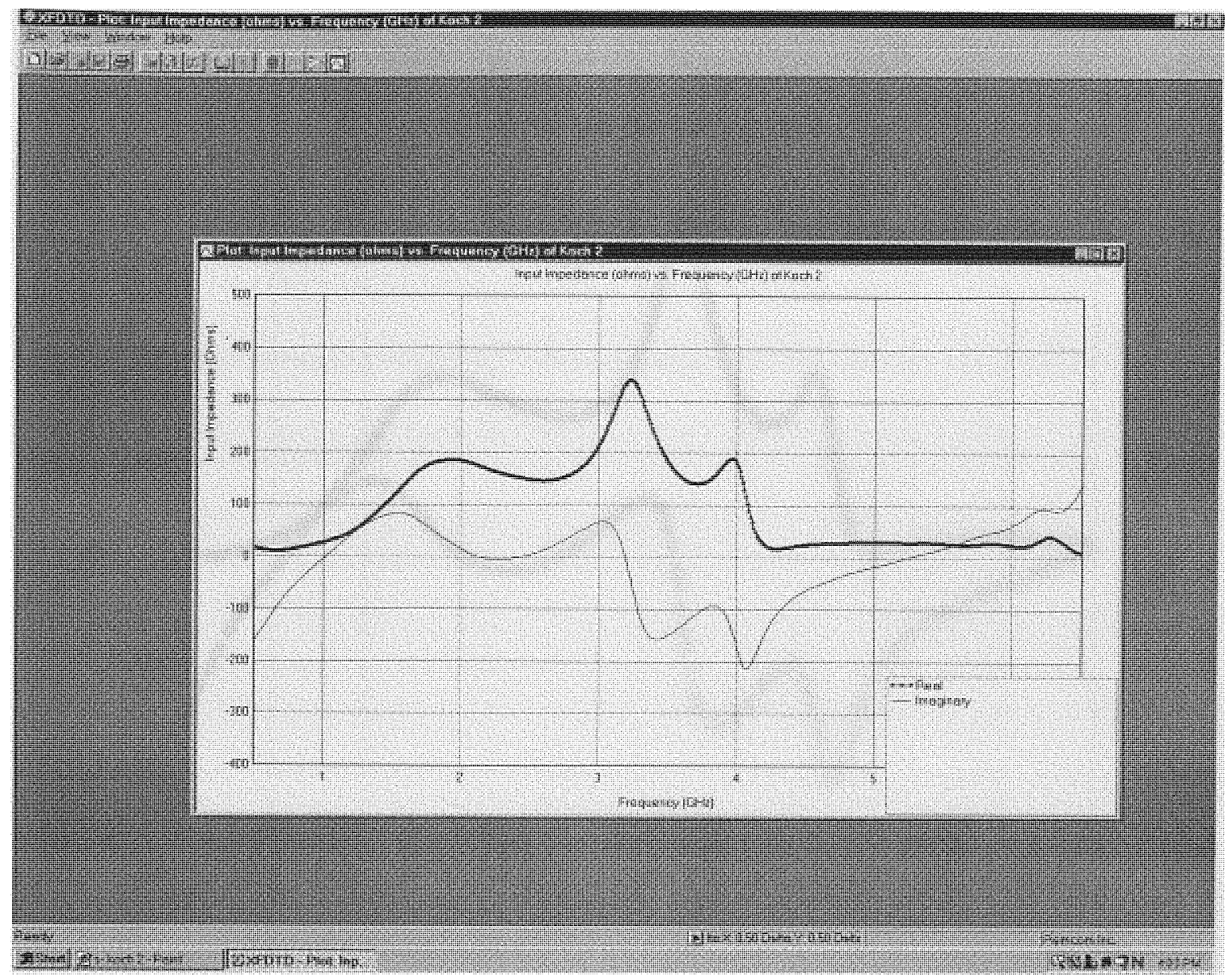

Figure 47 Input Impedance of Koch 2

Figure 47 shows the Imaginary and Real part of the input impedance of Koch 2 fractal. The results were simulated and obtained using FDTD platform. 
Figure 48 below shows the Imaginary and Real part of the input impedance of Koch 2 fractal.

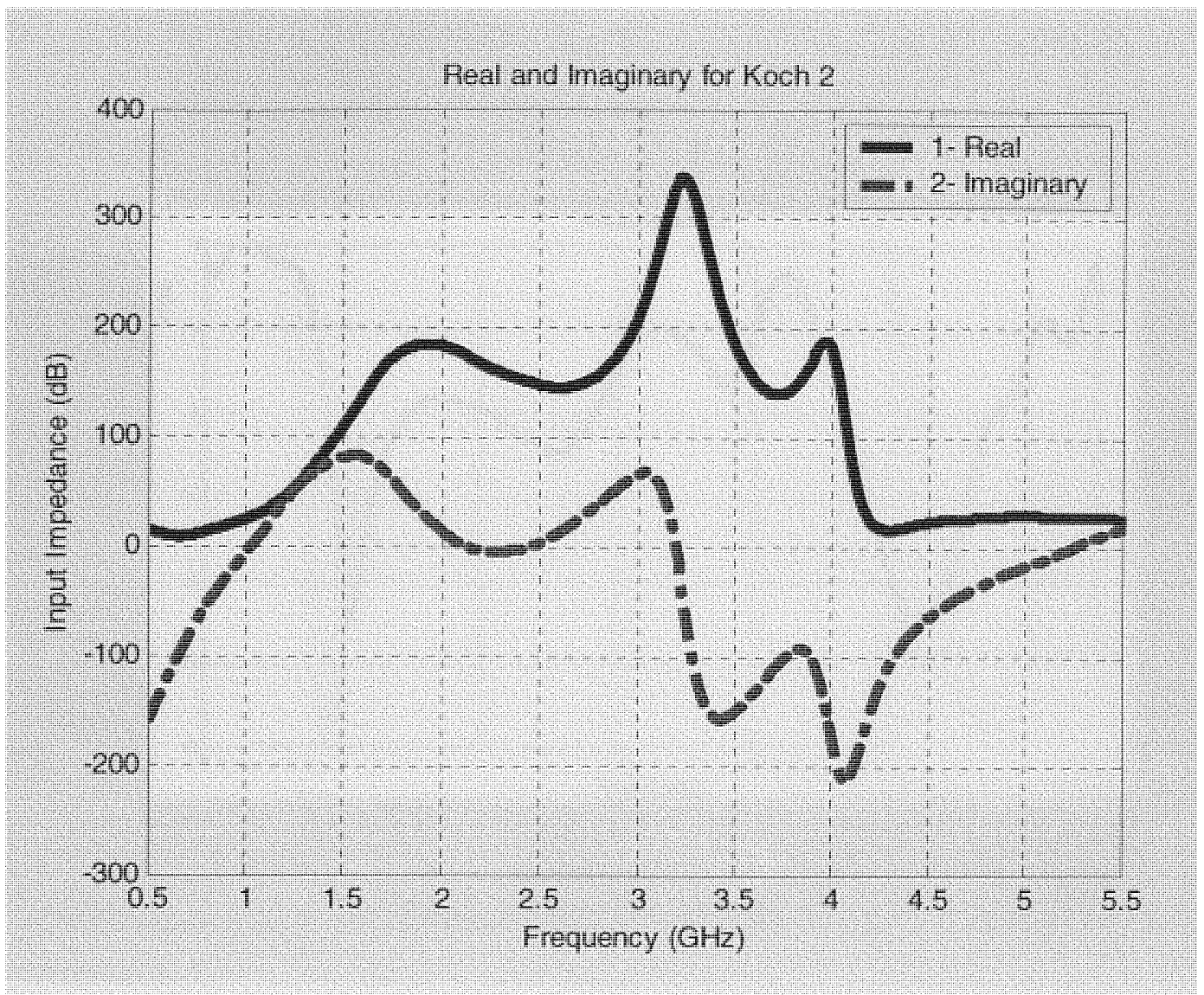

Figure 48 Real and Imaginary Input Impedance of Koch 2 


\subsection{FDTD $S_{11}$ Parameter plot for Koch 2}

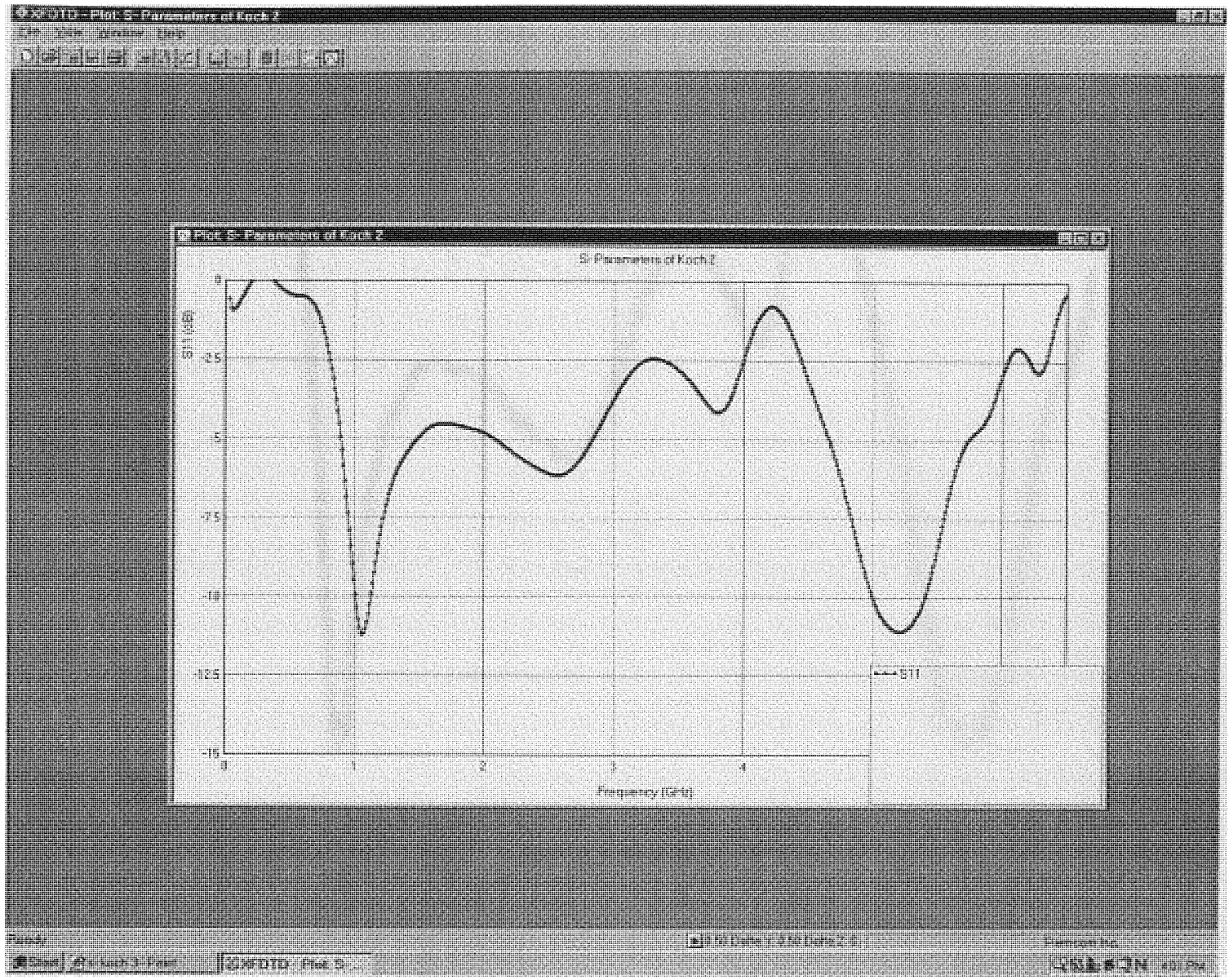

Figure $49 S_{11}$ Parameters for Koch 2 


\subsection{Return Loss for Koch 2}

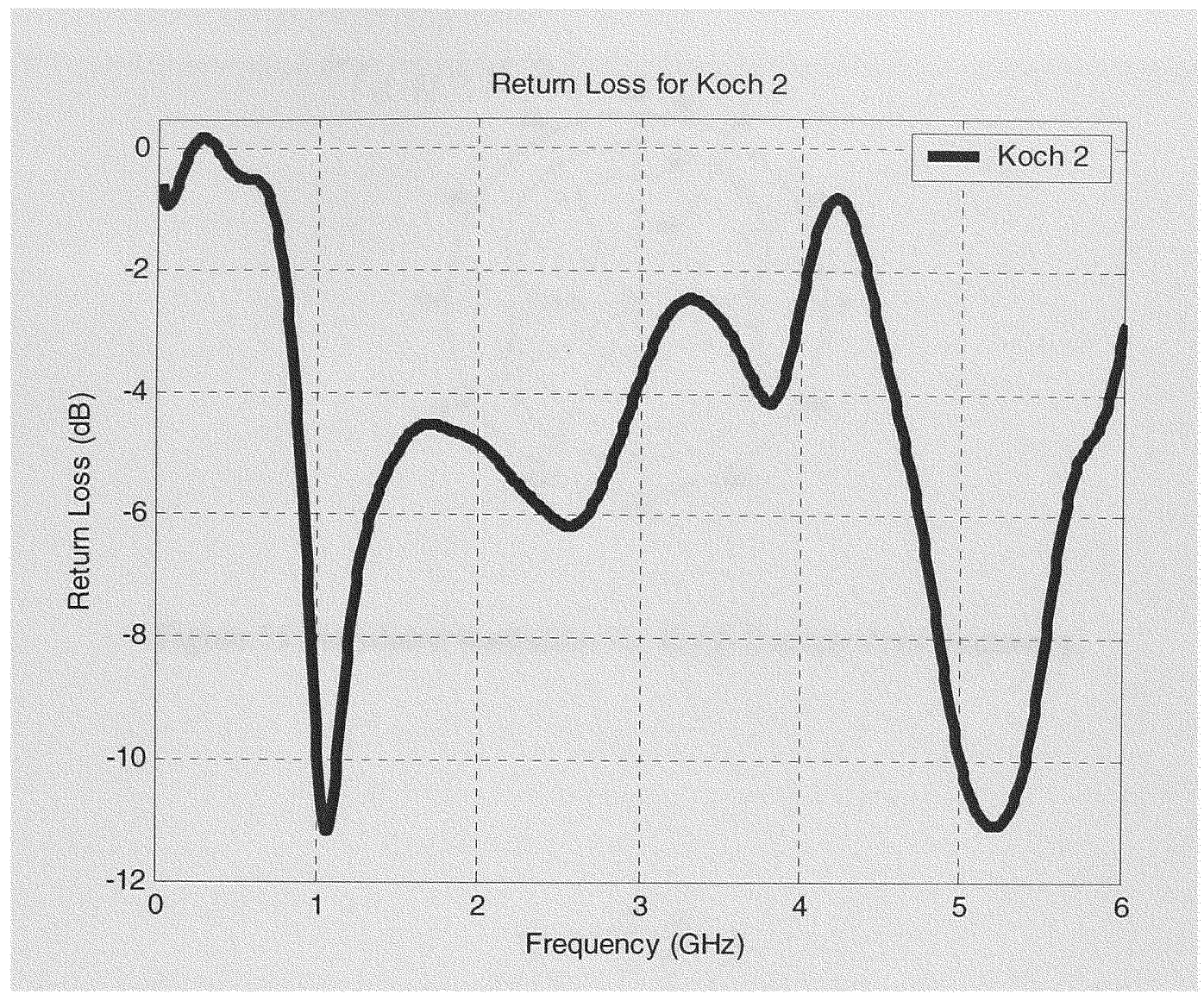

Figure 50 Return Loss of Koch 2

The input impedance bandwidth obtained from the numerical calculation has an operational bandwidth ranges from about $1.22 \mathrm{GHz}$ to $5.3 \mathrm{GHz}$.

At resonant frequency of $1.22 \mathrm{GHz}$ it is found that Koch 2 is to be $-11 \mathrm{~dB}$ and at $5.3 \mathrm{GHz}$ was $-10.9 \mathrm{GHz}$ 


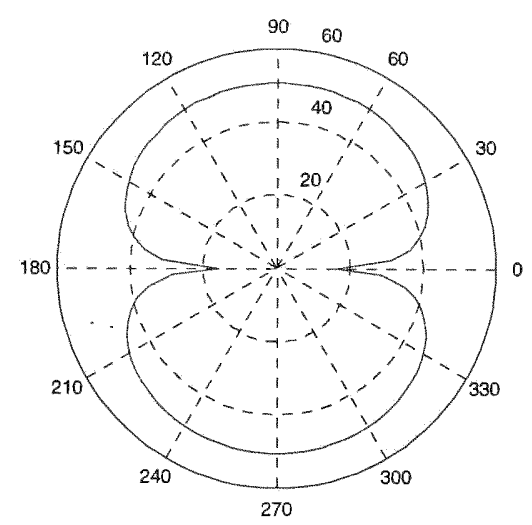

Figure 51 Simulated Radiation for Koch 2 at resonant frequency.

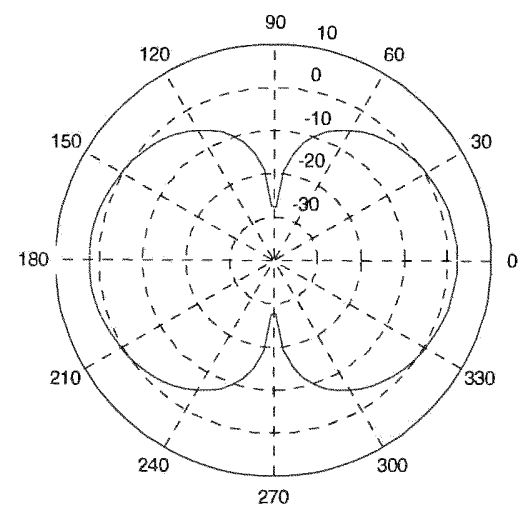

Figure 52 Simulated Radiation for Koch 2 at resonant frequency. 


\subsection{Koch 3 in XFDTD Space}

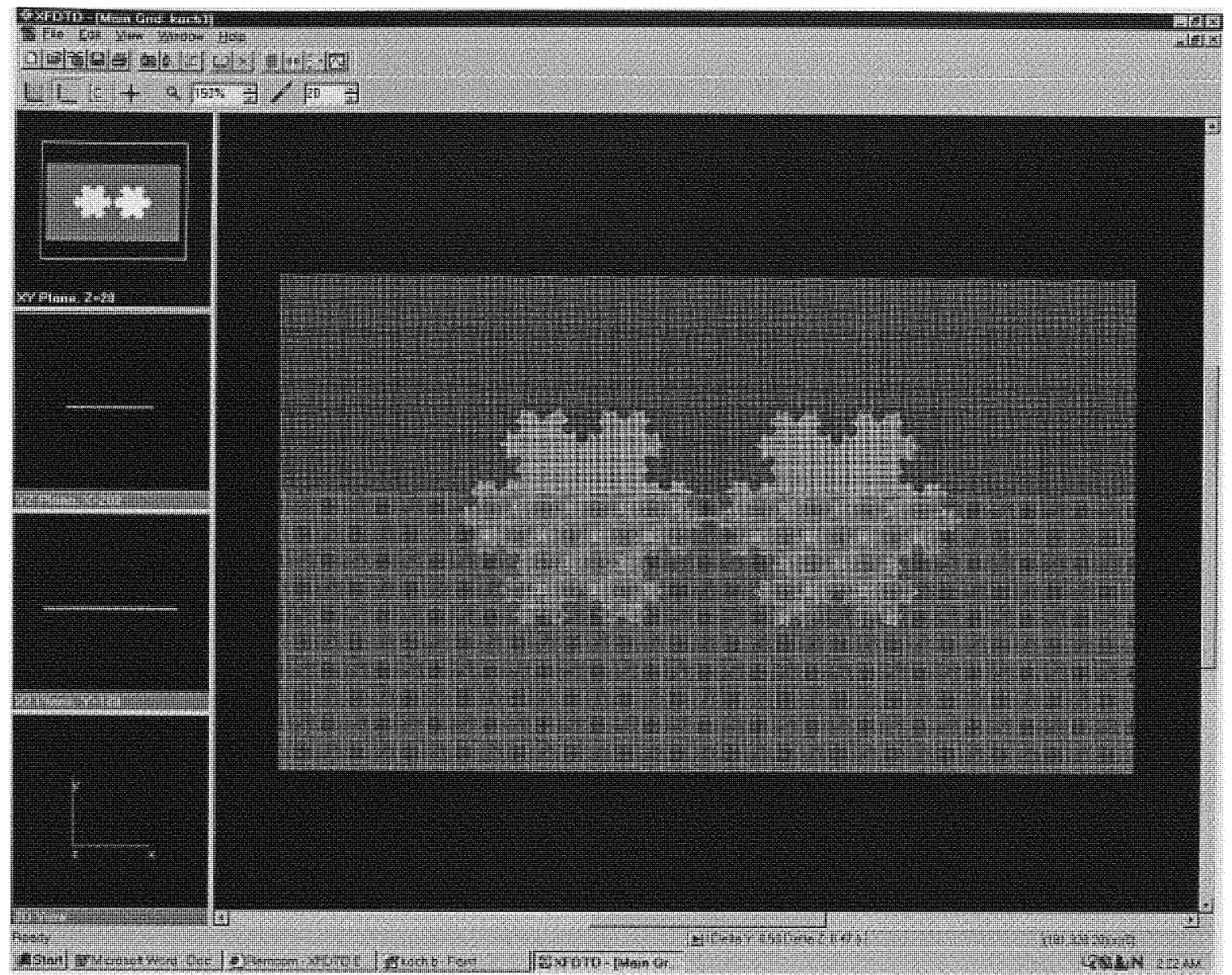

Figure 53 Koch 3 at XY plane in XFDTD space 


\subsection{FDTD Input voltage vs Frequency Plot of Koch 3}

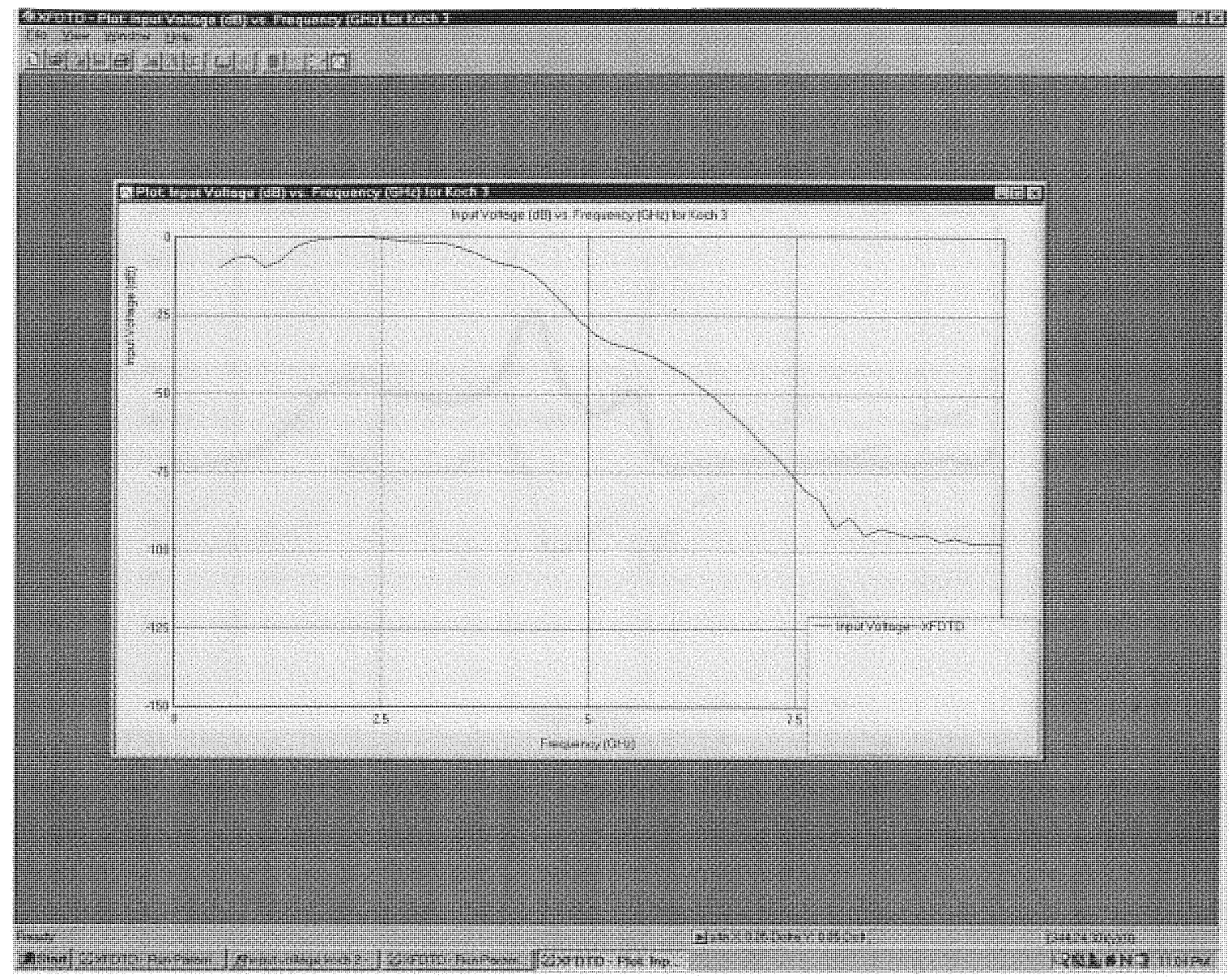

Figure 54 Input Voltage vs Frequency 


\subsection{XFDTD Input Impedance of Koch 3}

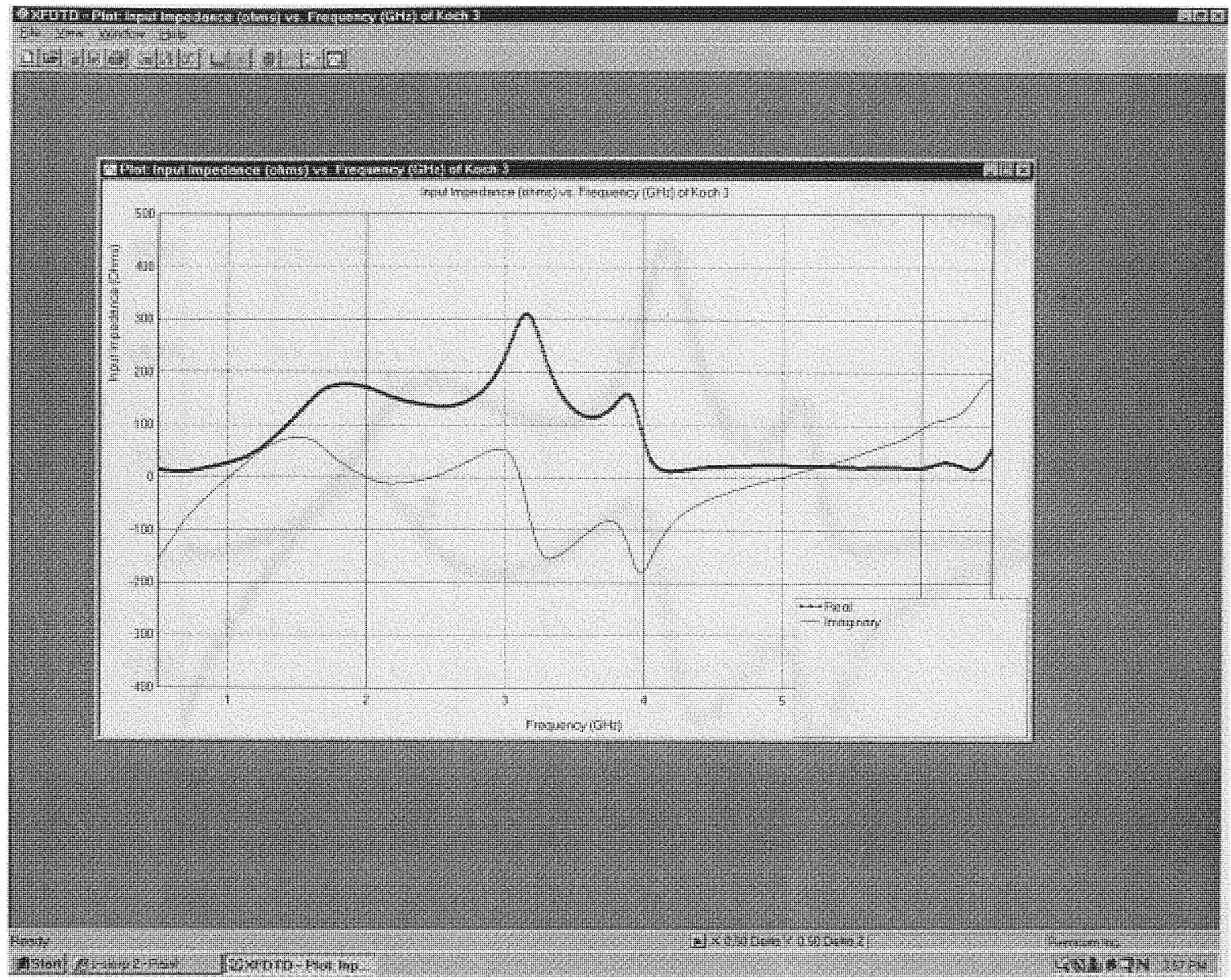

Figure 55 Input Impedance of Koch 3

Figure 55 shows the Imaginary and Real part of the input impedance of Koch 3 fractal. This result was simulated and obtained by using the FDTD platform. 
Figure 56 below shows the Imaginary and Real part of the input impedance of Koch 3 fractal.

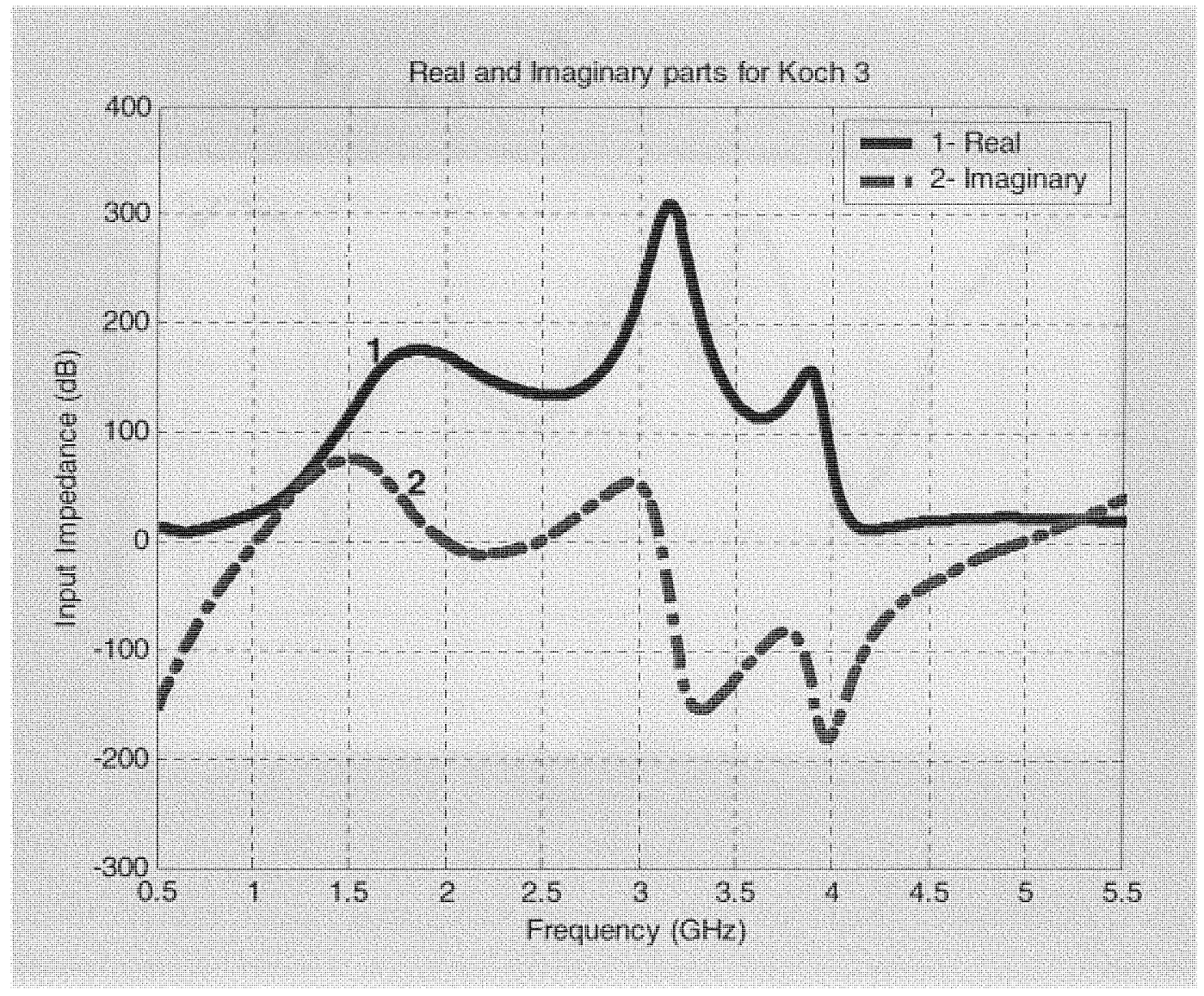

Figure 56 Input Impedance of Koch 3 


\subsection{FDTD $S_{11}$ Parameter Plot for Koch 3}

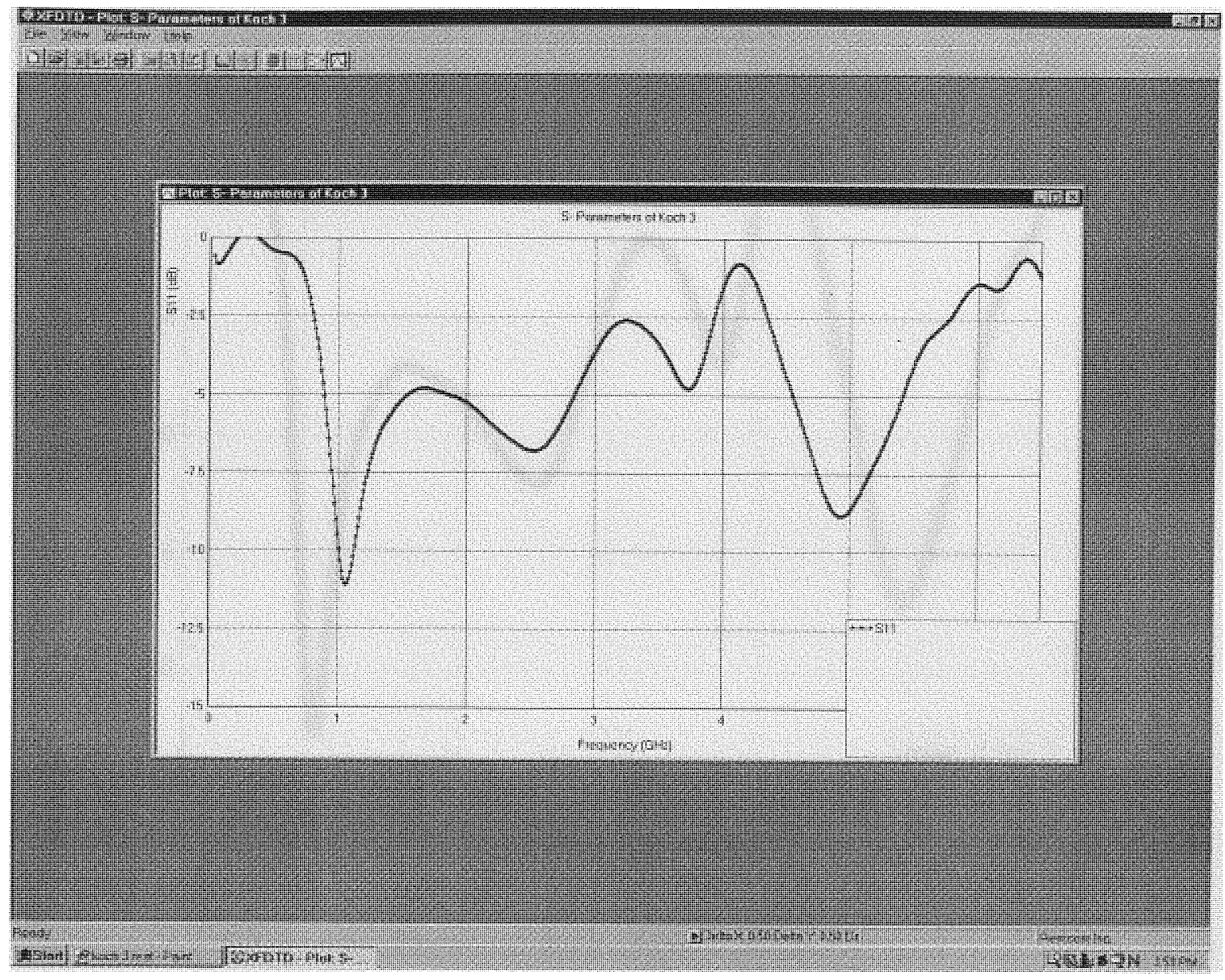

Figure $57 \mathrm{~S}_{11}$ Parameter of Koch 3 


\subsection{Return Loss for Koch 3}

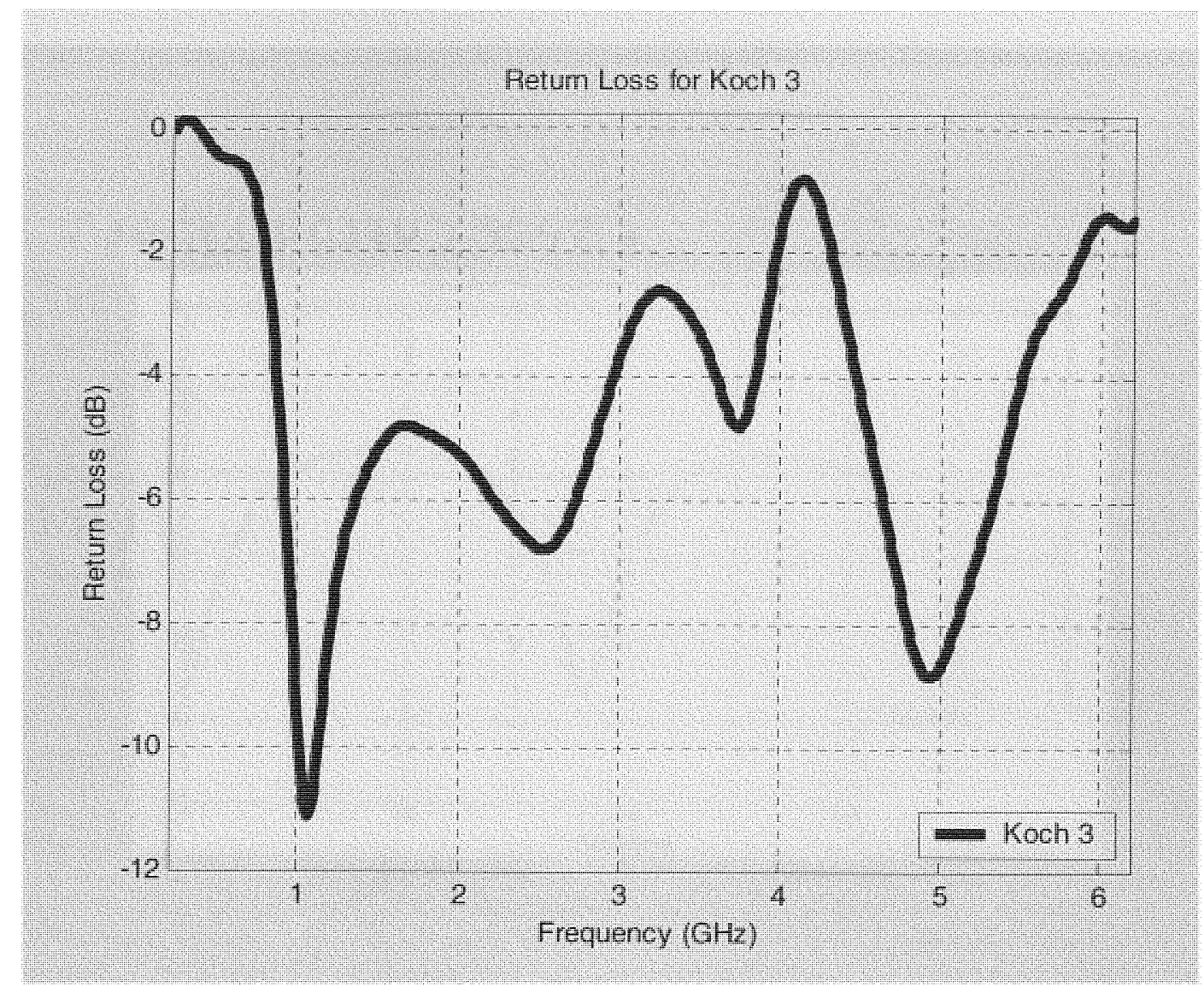

Figure 58 Return Loss of Koch 3

The figure above shows return loss of Koch 3 fractal. The input impedance bandwidth obtained from the numerical calculation has an operational bandwidth ranges from about $1.22 \mathrm{GHz}$ to $4.9 \mathrm{GHz}$.

At resonant frequency of $1.22 \mathrm{GHz}$ it is found that Koch 3 is to be $10.9 \mathrm{~dB}$ 


\subsection{XFDTD Gain Plot for Koch 3}

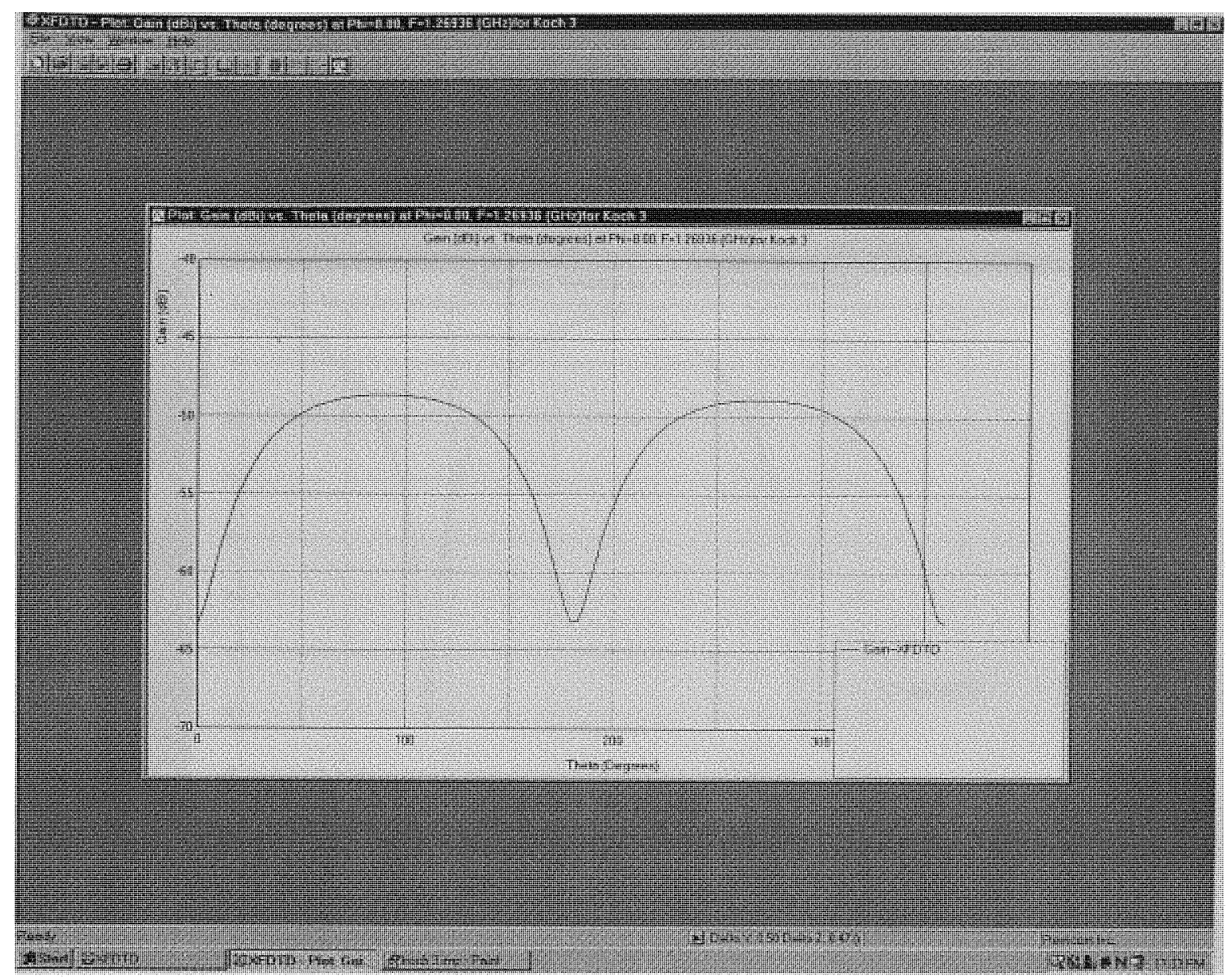

Figure 59 FDTD Simulated Gain Koch 3

The figure above shows the Gain vs Angle FDTD simulation when Phi is constant. 


\subsection{XFDTD CP Gain Plot for Koch 3}

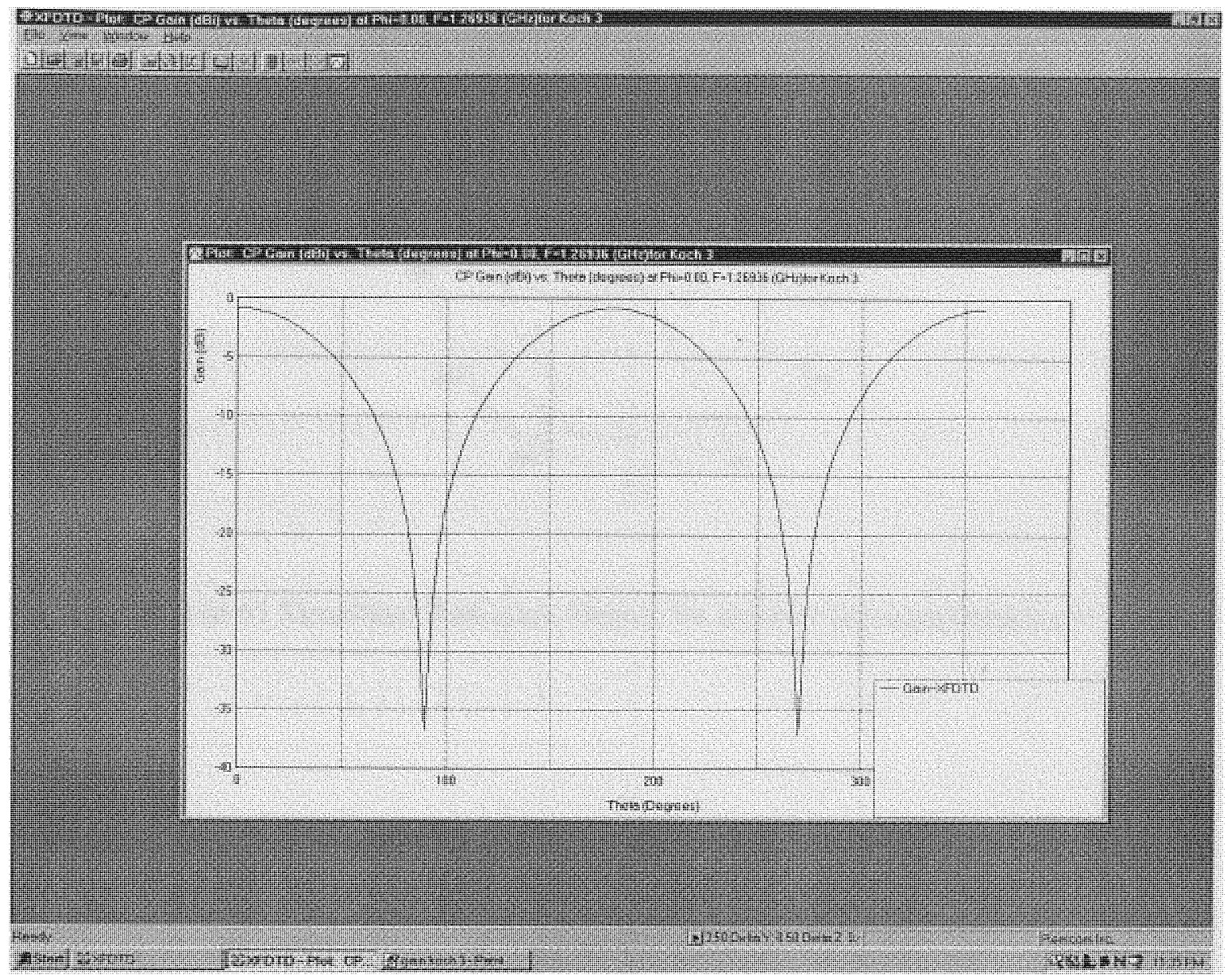

Figure 60 FDTD Simulated CP Gain Koch 3

The Figure above shows the Gain vs Angle XFDTD simulation at Constant Phi. In this simulation Phi is set to zero. 


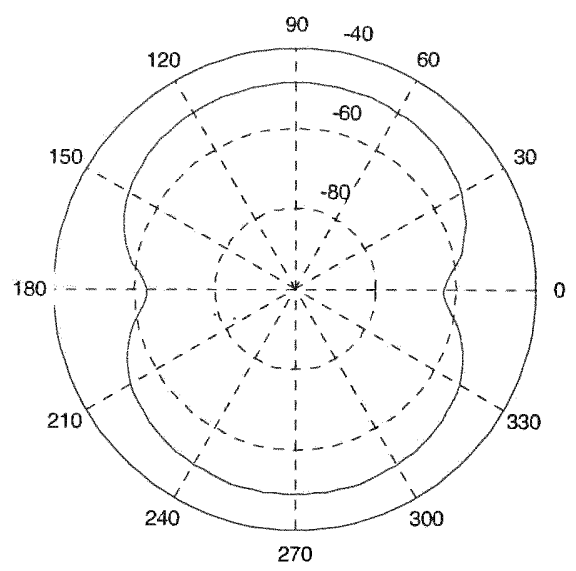

Figure 61 Simulated Radiation Pattern for Koch at resonant frequency.

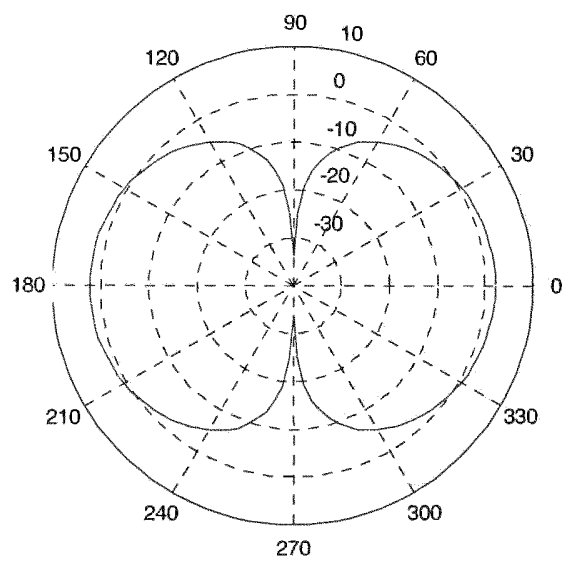

Figure 62 Simulated Radiation Pattern for Koch at resonant frequency. 


\subsection{Results / Discussion}

In this thesis, the Koch Snowflake Dipole antenna was simulated and analyzed using the XFDTD numerical method. The antenna was designed having 4 iteration stages. These were implemented on the XFDTD platform and analyzed accordingly. The necessary parameters as stated earlier in the chapter were setup to carry out the simulation.

From the XFDTD numerical analysis of the Koch snowflake dipole antenna, it could be deduce that the bandwidth of the antenna structure increases as the number of iteration stages increases. It could also be seen that the resonant frequency of the four geometries studied remains the same. It should be noted that with an increase in the number of iterations, the Koch snowflake antenna shows an increase in the quality factor $Q$ of the antenna.

In analyzing the numerical results it was observed that the resonant frequency $S_{11}$ response of the first iteration stage at Koch 0, was found to be $-19 \mathrm{~dB}$. Further analysis shows that at the next stage at Koch 1 the $S_{11}$ response was $-19 \mathrm{~dB}$. For iteration stage, Koch 2 the $S_{11}$ response was found to be $-10.9 \mathrm{~dB}$ and at the final iteration stage at Koch 3 the $\mathrm{S}_{11}$ was $-10.9 \mathrm{~dB}$.

With these analyses it can be seen that at each iteration stages the Koch Snowflake antenna yields a different $S_{11}$ responses. That is, at Koch 0 the antenna exhibits a $S_{11}$ response of $-3 \mathrm{~dB}$, at Koch 1 it has an $S_{11}$ of $-3.97 \mathrm{~dB}$, at Koch2 the antenna has an $\mathrm{S}_{11}$ of $-4.56 \mathrm{~dB}$ and for the last iteration stage Koch3 it has an $\mathrm{S}_{11}$ of $-4.82 \mathrm{~dB}$. 
The figure below shows Return Loss of Koch Antenna with Various Iterations

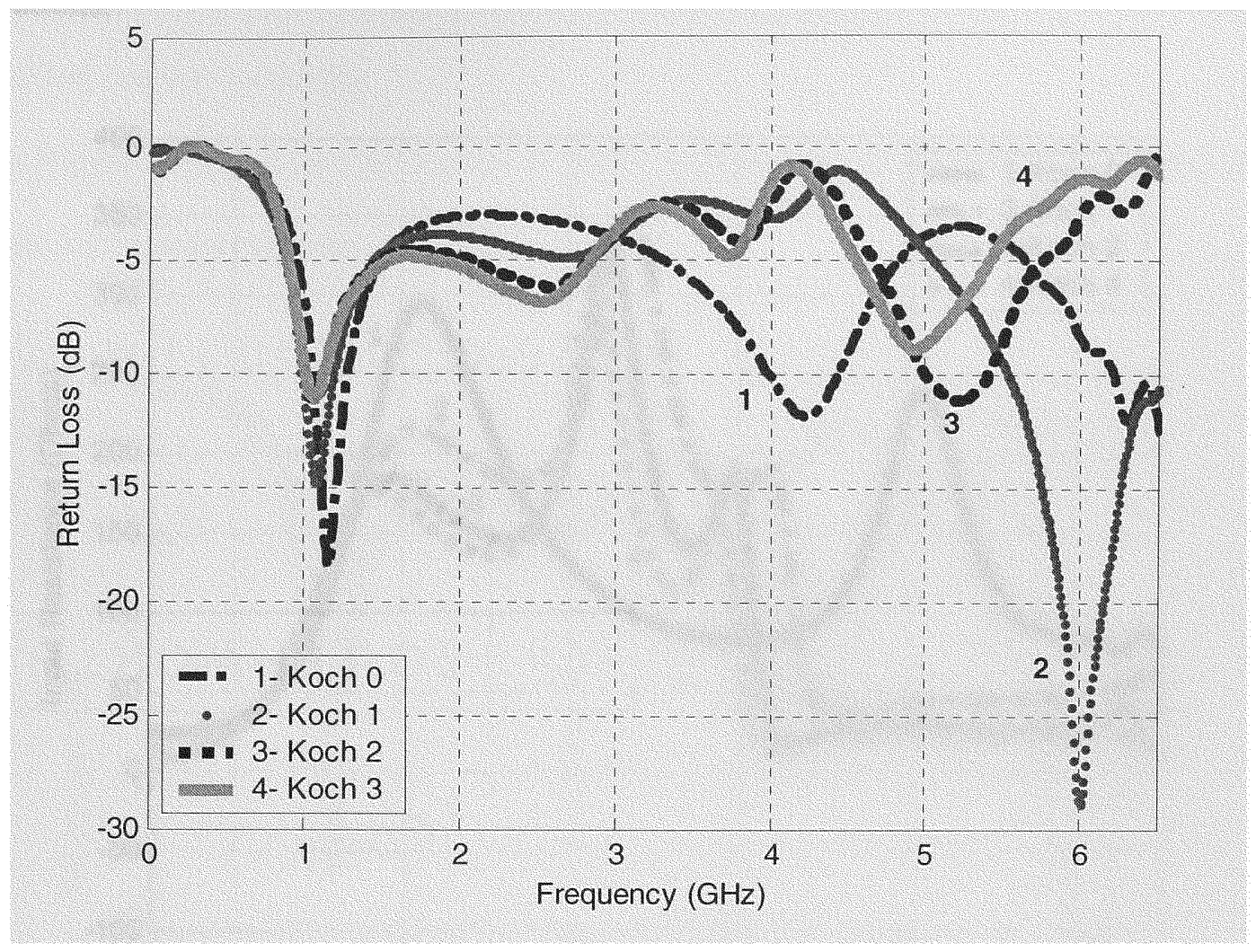

Figure 63 Return Loss of Koch Antenna with Various Iterations

Further analysis of the XFDTD numerical results show that the input impedance relating to the four iteration stages converges as the number of stages increases. In analyzing the radiation plots of each iterated structure, it was seen that for fractal iteration for the radiation pattern was constant throughout each stage and thus remains the same. However, a slight change in the third fractal iteration stage was observed in which the null was removed. 
The figure below shows the variation in input impedance for the Koch fractal at various iterations.

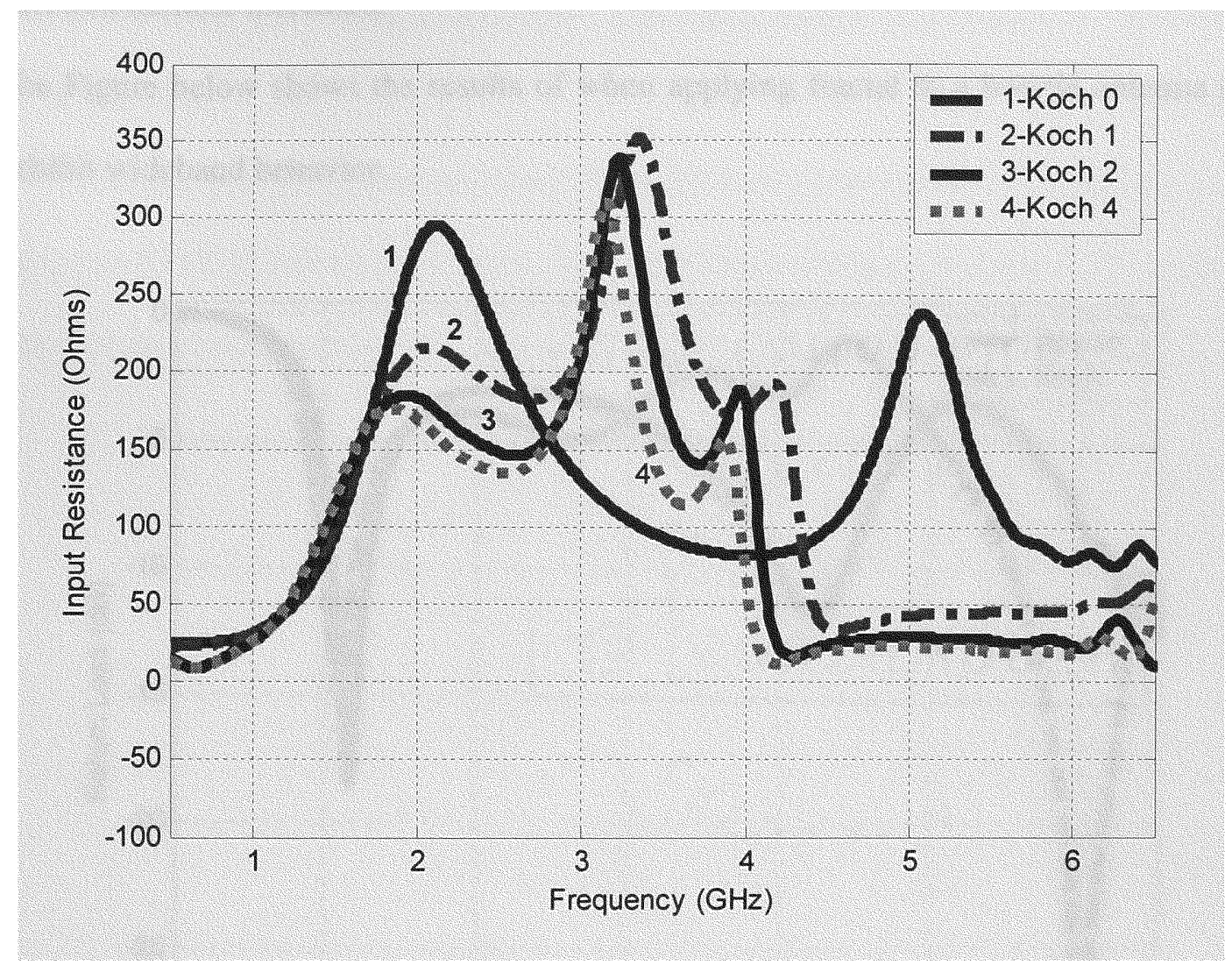

Figure 64 Input Resistance for Koch Fractal with different iterations

It can be clearly seen that the input impedance of the Koch fractal varied according to different iterations. This is a very useful characteristic because by adjusting the iteration stages we were able to control a very important property of the antenna. It is seen that as the number of iterations increases the input impedance converges. 
It can be said that the Koch Snowflake antenna exhibit lower resonant frequencies as the number of fractal iteration increases. The antenna has a wideband property as the iteration number increases.

The Figure below shows the results of when applying fractal to a bowtie antenna it can exhibit wideband behavior.

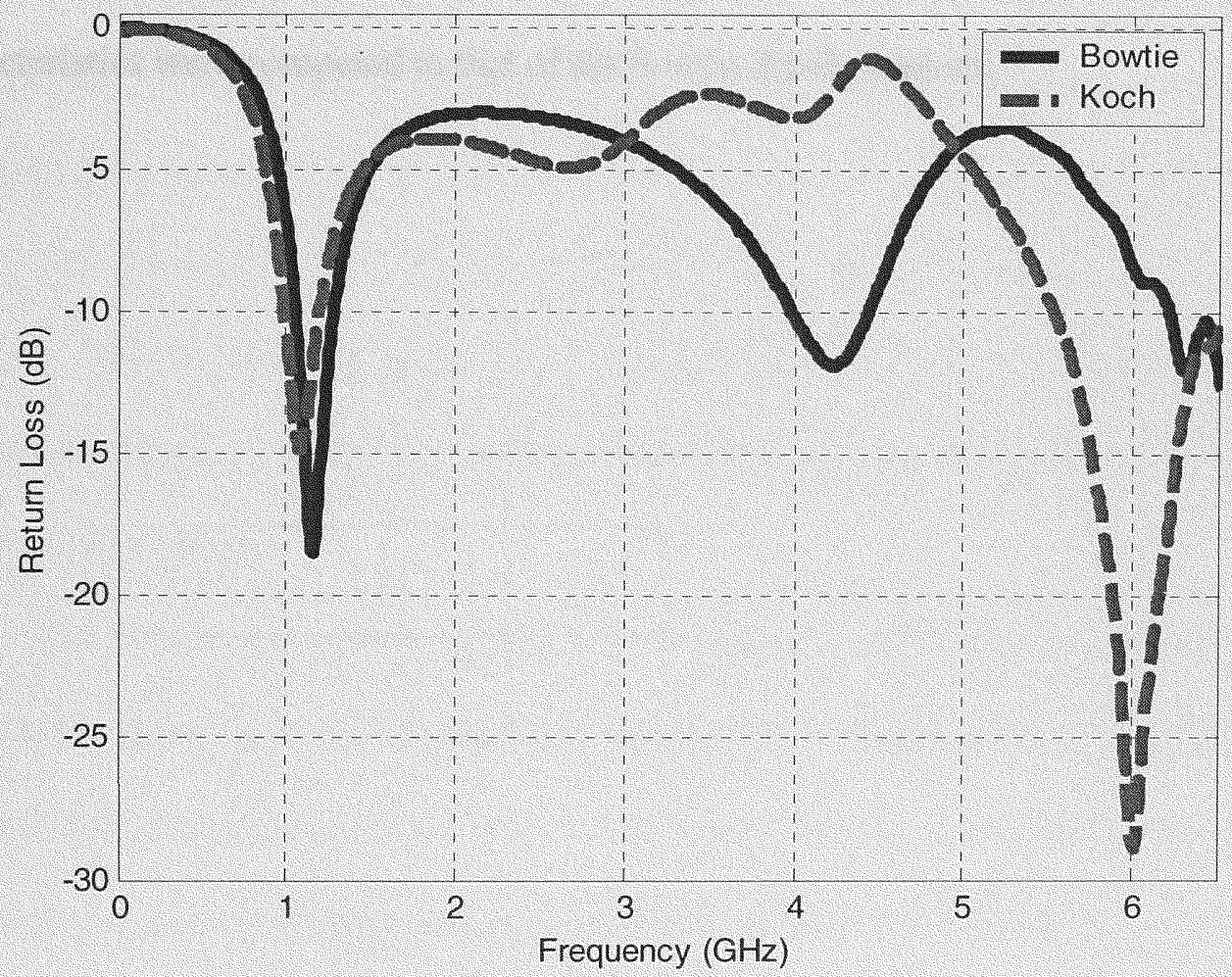

Figure 65 Return Loss for Bowtie and Koch Fractal

From the numerical calculation it can be seen that at the stage of iteration Koch 1 fractal the antenna shows an increase in bandwidth. At S11 response the resonant frequency of $1.23 \mathrm{GHz}$ is to be $-15 \mathrm{~dB}$ and at $6 \mathrm{GHz}$ the return loss was $-29 \mathrm{~dB}$. 
The numerical calculation shows that the Koch 1 has an operation bandwidth from ranges $1.23 \mathrm{GHz}$ to $6 \mathrm{GHz}$. While the operational bandwidth for the Bowtie antenna was found to be $1.23 \mathrm{GHz}$ to $4.2 \mathrm{GHz}$. This result shows an increase in bandwidth of about $1.8 \mathrm{GHz}$.

In this thesis the proposed Koch Snowflake structure was developed and analyzed using the XFDTD numerical method. The results were observed and the performance and characteristics were compared to that of the bowtie dipole antenna. 


\section{Chapter 7 FDTD Analysis of Sierpinski Gasket Fractal Antenna}

\subsection{Introduction}

The Sierpinski gasket is one of the most widely studied fractal geometry for antenna applications. The Sierpinski gasket antennas have been researched considerably for monopole and dipole antenna configurations [19]. The self-similar current distribution on these antennas is expected to cause its multi-band characteristics [20]. That is, the antenna has shown various frequencies when operating. It has been found that by changing the geometry the multi-band nature of these antennas can be controlled [21].

The objective of this chapter is to show how fractals can be used as multiband antennas. This is done by simulating a Sierpinski gasket antenna using the XFDTD numerical method. It should be noted that the Sierpinski antenna can be compared to the Bowtie dipole antenna.

The Sierpinski gasket fractal is studied in this thesis to investigate the performance as an antenna with multiple frequencies. This antenna is said to have several bands of resonance due to its self-similarity of the fractal geometry. The Sierpinski gasket fractal antenna that is investigated in this thesis is implemented as a dipole. This structure is basically is scaled version to the $4^{\text {th }}$ iteration of a bowtie antenna. This design was chosen so as to compare its multiband characteristics with that of a bowtie antenna. 


\subsection{Geometry of Sierpinski gasket fractal}

As stated above, the Sierpinsk gasket fractal is a self-similar antenna that exhibit multiband characteristics [22]. In this thesis study, the Sierpinski gasket dipole will be analyzed. This antenna is very similar to that of a conventional bowtie antenna. Below in Figure 66 is the fractal iteration in which the Sierpinski gasket is generated. It can be seen that the first geometry is that of a bowtie antenna. The other geometries were generated from this initiator (triangle 1) and thus create the final Sierpinski triangle after the iteration stages have completed.

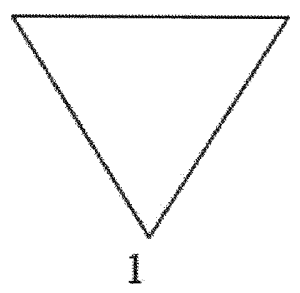

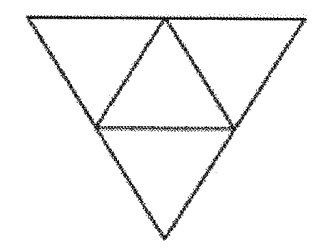

2

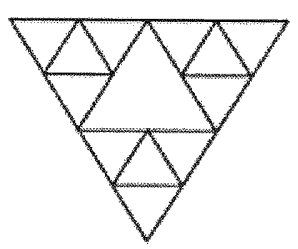

3

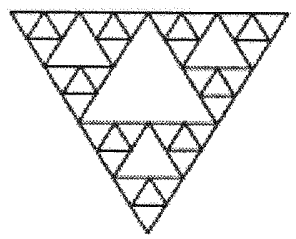

4

Figure 66 Iteration of a Sierpinski Gasket Fractal Antenna [24]

The Sierpinski triangle is constructed by repeatedly removing a central inverted equilateral triangles from an initial equilateral triangle of unit side length (Fig 66). Once the central inverted triangle is removed, three equilateral triangles remain on the geometry, each with half the size of the initial triangle.

This procedure can be looked at as repeatedly replacing an equilateral triangle by three triangles on half the height [24]. 
This iteration is carried out an infinite number of times until the desired fractal Sierpinski gasket is obtained. In this thesis, a $4^{\text {th }}$ order iteration stage was used to test and analyzed the multiband properties of the antenna.

By applying several slots on the patch surface of the conventional bowtie dipole antenna forms the fractal like bowtie dipole antenna as shown in Figure 67. With this structure, each triangle of its three main parts are exactly equal and identical to the whole geometry, but it is scaled by a factor of two and so are each of the three triangle gasket that make up any of those parts.

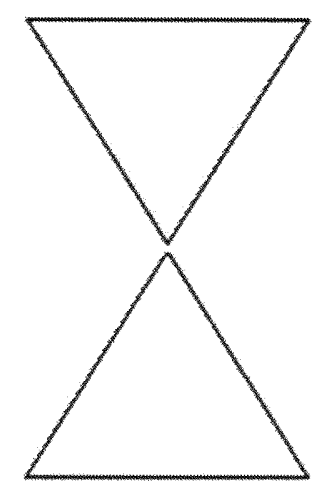

Sierpinsld 0

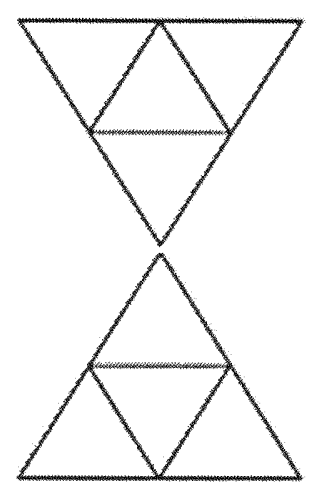

Sierpinsul 1

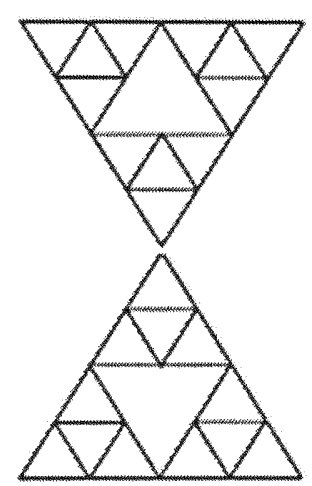

Sucrounus 2

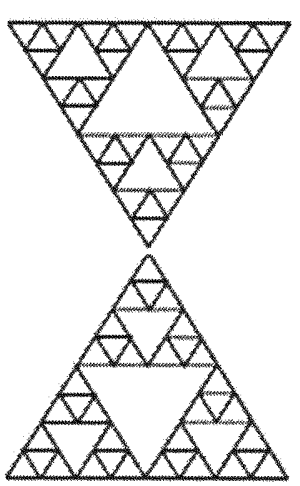

sierpinsidi

Figure 67 Geometry of Sierpinski Gasket Dipole Antenna [24]

In this study, the Sierpinski gasket antenna is implemented as a dipole antenna as shown in Figure 67. 
The antenna source is fed as a dipole at the center with a Modulated Gaussian

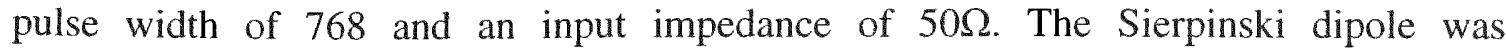
constructed and measured on the XFDTD platform.

The antenna was mounted on a FR-4 substrate with a relative dielectric constant $\varepsilon_{\mathrm{r}}=4.7$. The thickness of the substrate is $0.01 \lambda$.

The antenna was modeled using the FDTD model. The 3 dimensional FDTD space was determined. The FDTD computational space has $200 \times 180 \times 20$ cells, with a space step of $\Delta \mathrm{X}=0.05 \mathrm{~mm}, \Delta \mathrm{Y}=0.05 \mathrm{~mm}$ and $\Delta \mathrm{Z}=0.047 \mathrm{~mm}$.

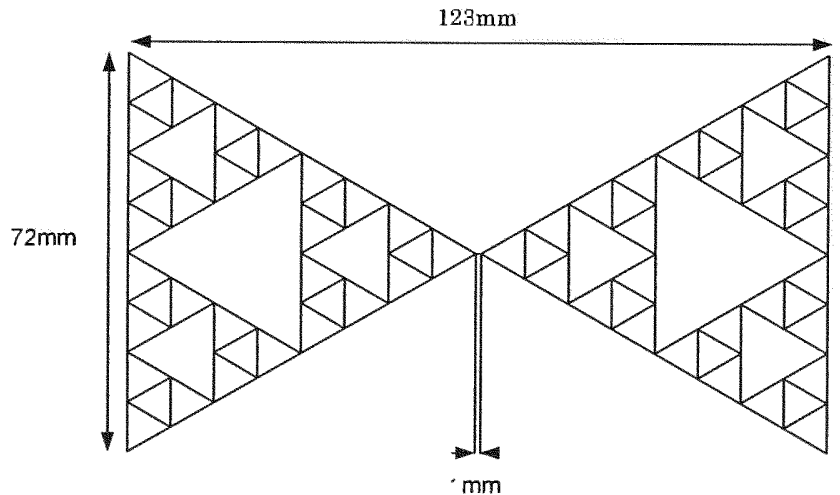

Figure 68 Geometry of Sierpinski Fractal 


\subsection{Results of the Siepinski Gasket Fractal Antenna XFDTD Analysis}

In this thesis a Sierpinski Gasket Dipole antenna is presented. As stated above, this antenna structure will be implemented and simulated using the XFDTD numerical platform. The results are compared to the original Bowtie dipole antenna in which it was created from.

The each iteration stage of the antenna structure was simulated and the results were analyzed so see that changes in characteristics and performance for each iteration stages. The results of the XFDTD analysis of the Sierpinski Gasket are presented in the following pages, pp 100-135 


\subsection{XFDTD Run Parameter for Sierpinski Gasket 0}

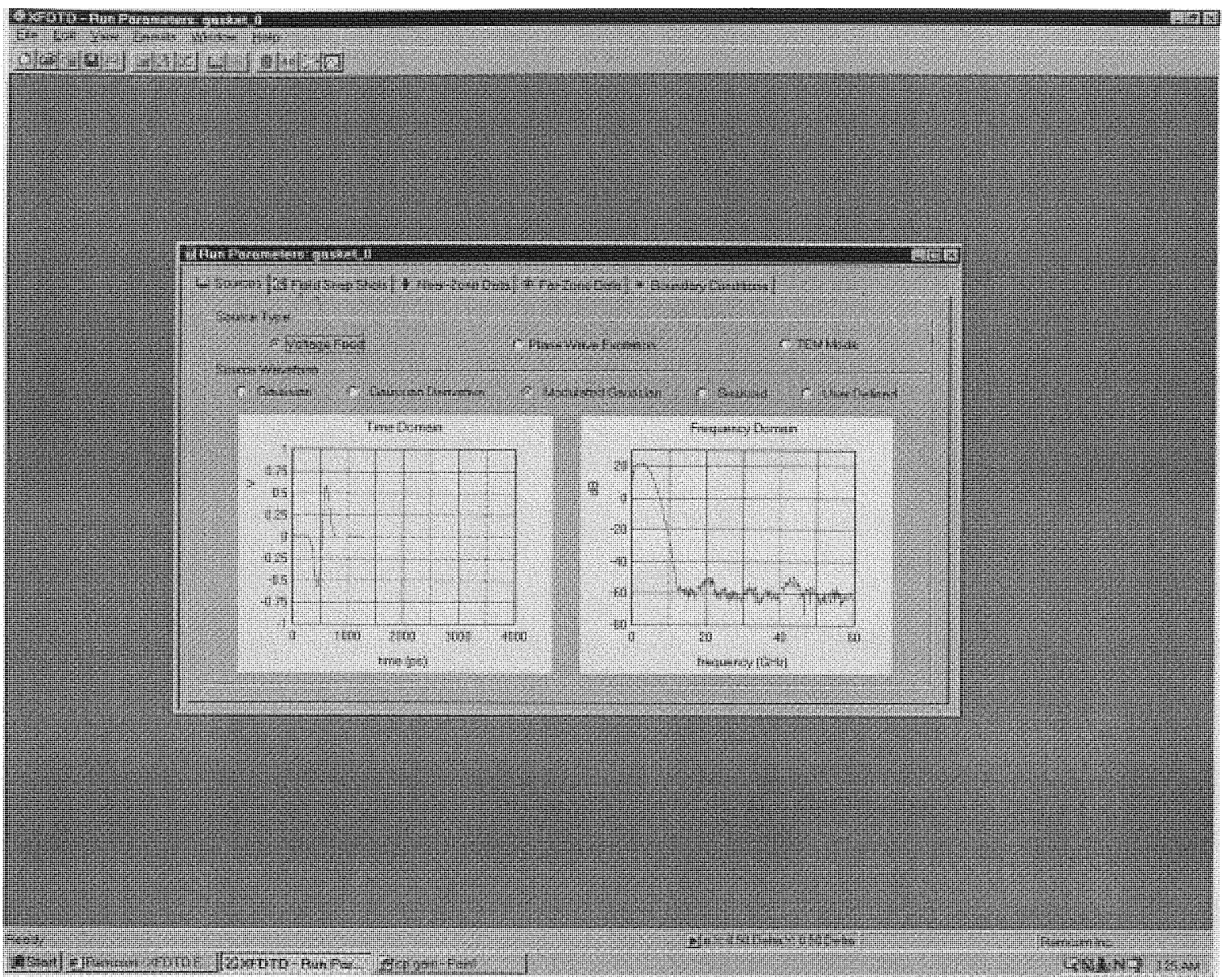

Figure 69 FDTD setup Run Parameters for Siepinski Gasket 0

Figure 69 shows the setup run parameters for a FDTD simulation. The Modulated

Gaussian was implemented in the setup. 


\subsection{Sierpinski 0 in XFDTD Space}

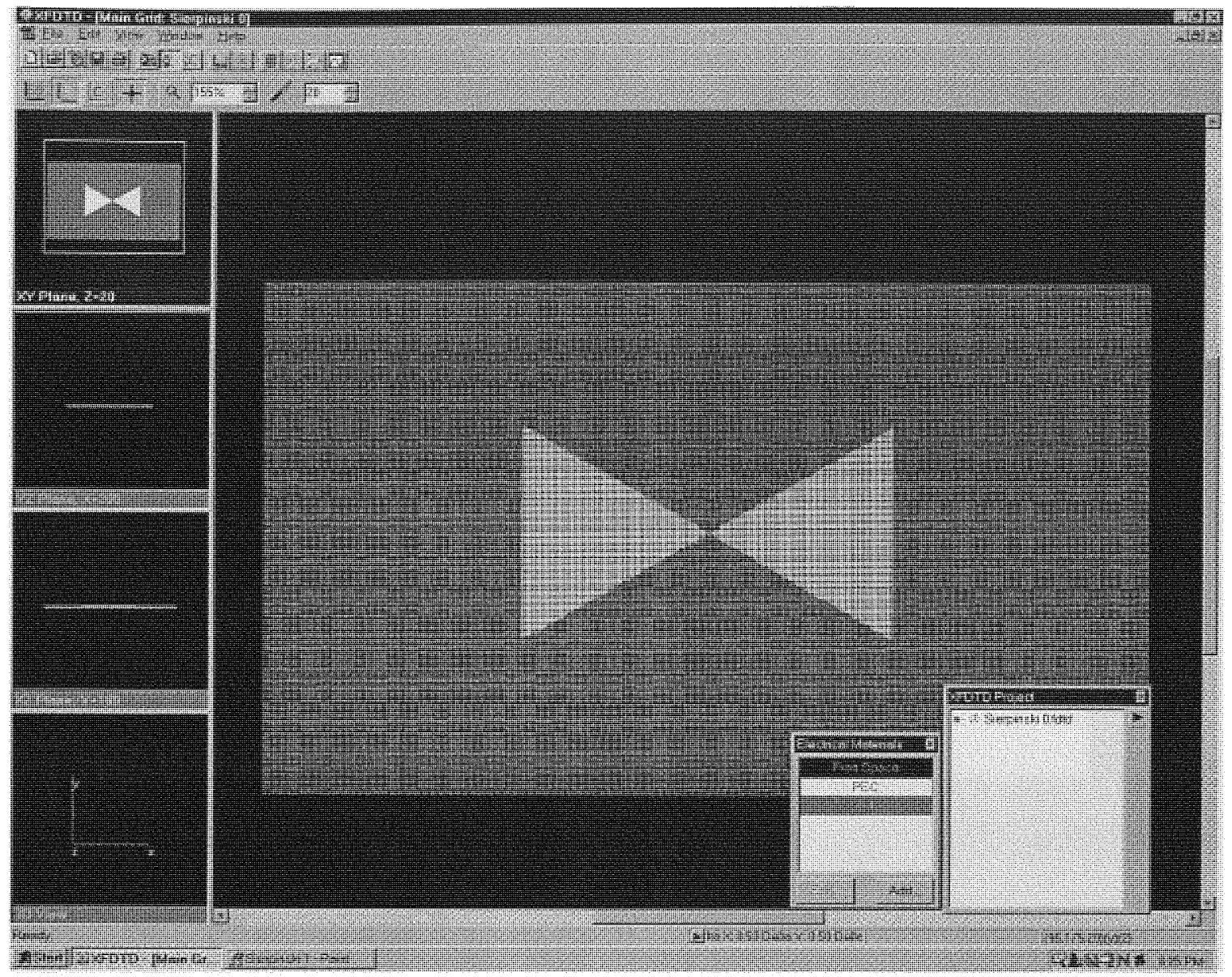

Figure 70 Sierpinski 0 at XY Plane in XFDTD Space 


\subsection{XFDTD Input Impedance plot of Sierpinski 0}

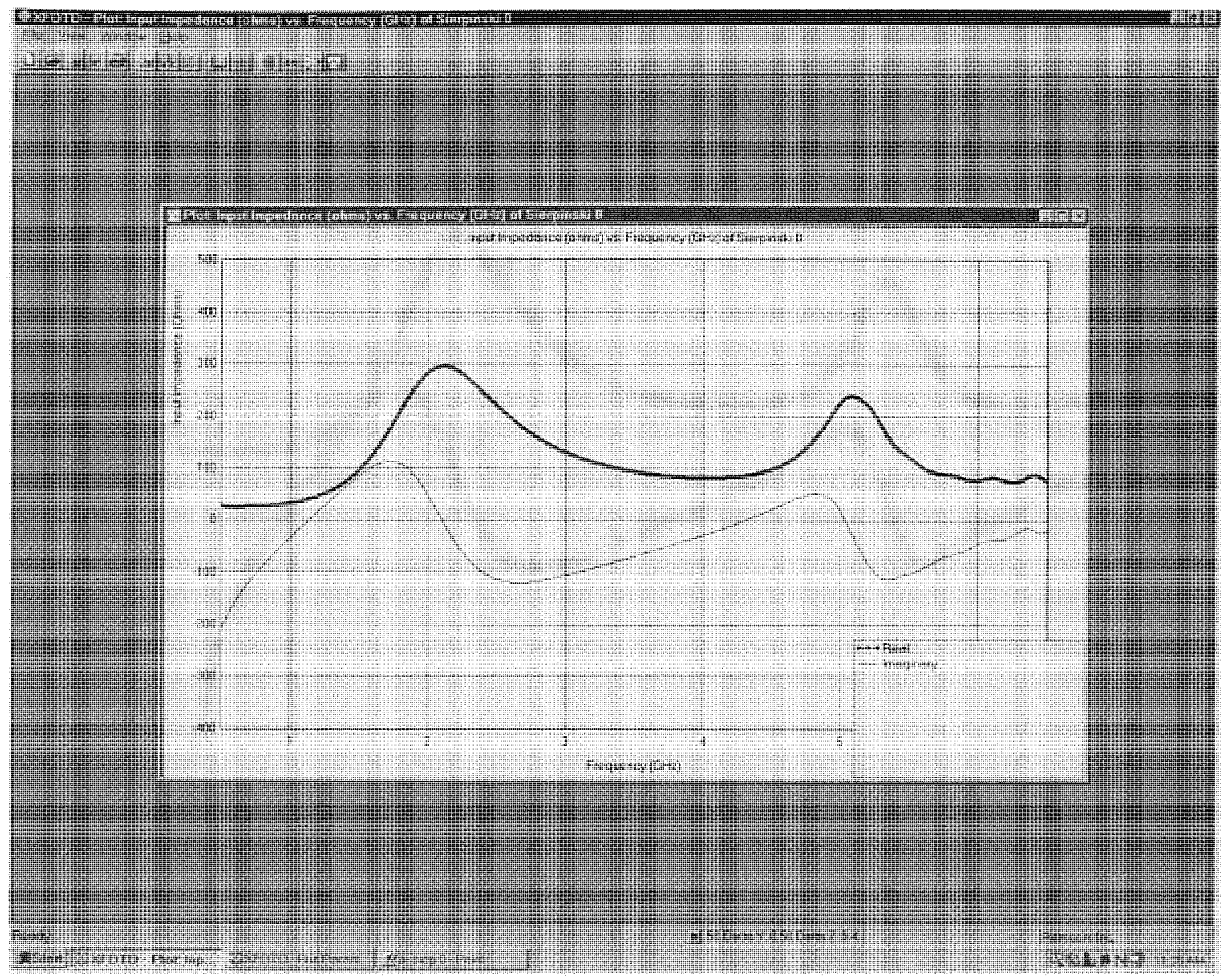

Figure 71 Input Impedance of Sierpinski 0

Figure 71 shows the Imaginary and Real part of the input impedance of the Sierpinski 0 fractal. The results were obtained from the FDTD simulation. 
Figure 72 below shows the Imaginary and Real part of the input impedance of the Sierpinski 0 fractal

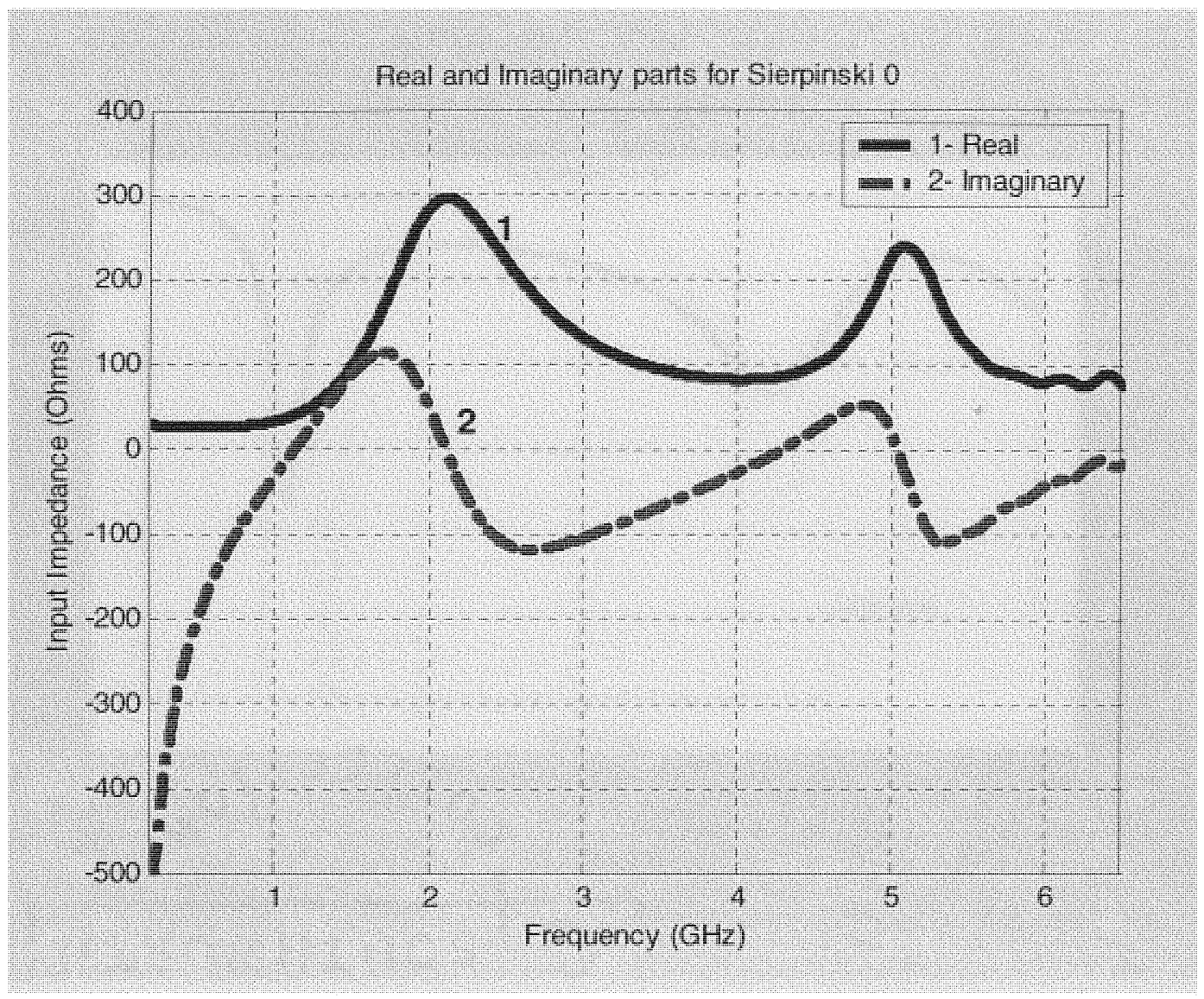

Figure 72 Real and Imaginary Input Impedance of Sierpinski 0 


\subsection{XFDTD $S_{11}$ Parameter Plot of Sierpinski 0}

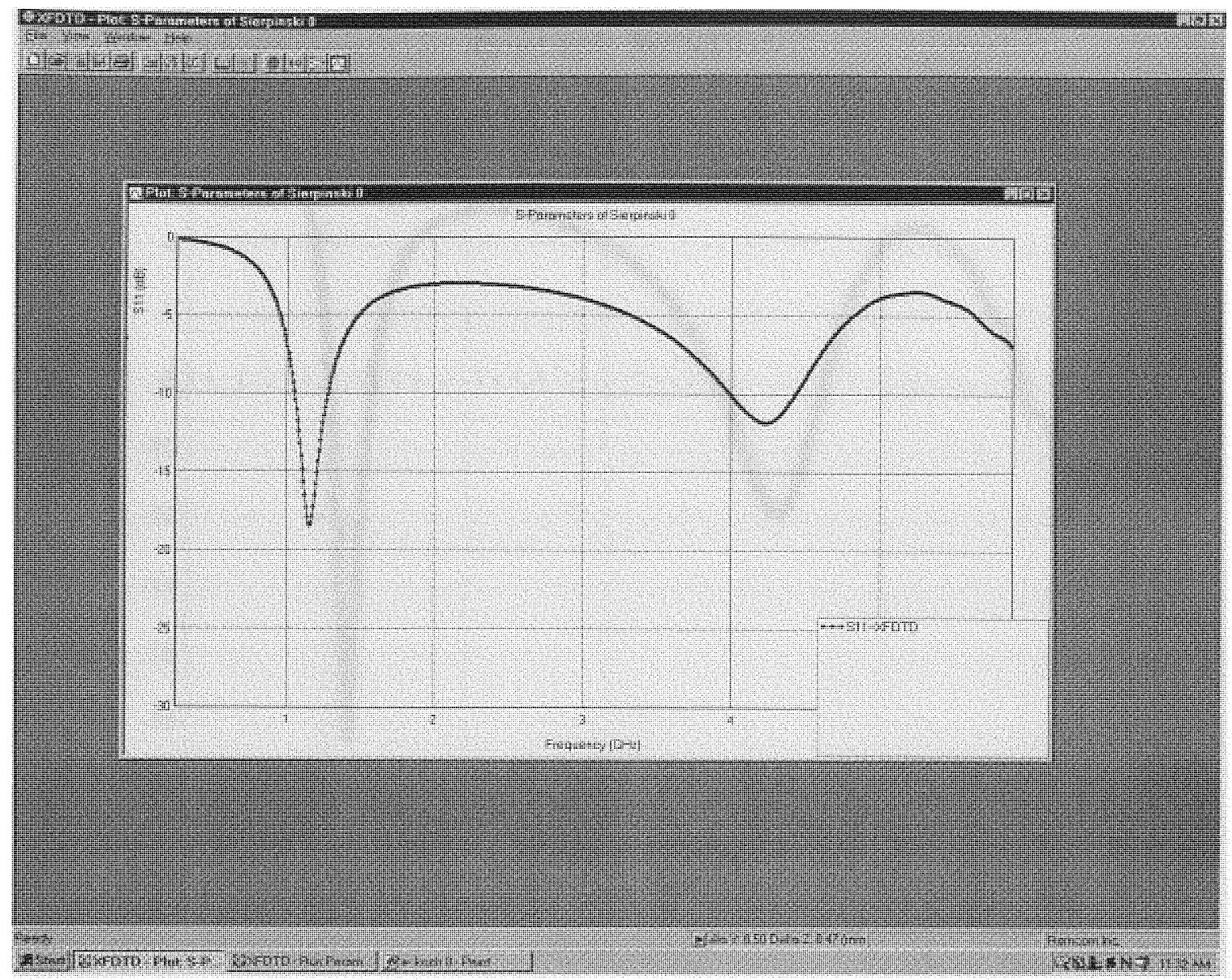

Figure $73 S_{11}$ Parameters of Sierpinski 0 


\subsection{Return loss for Sierpinski 0}

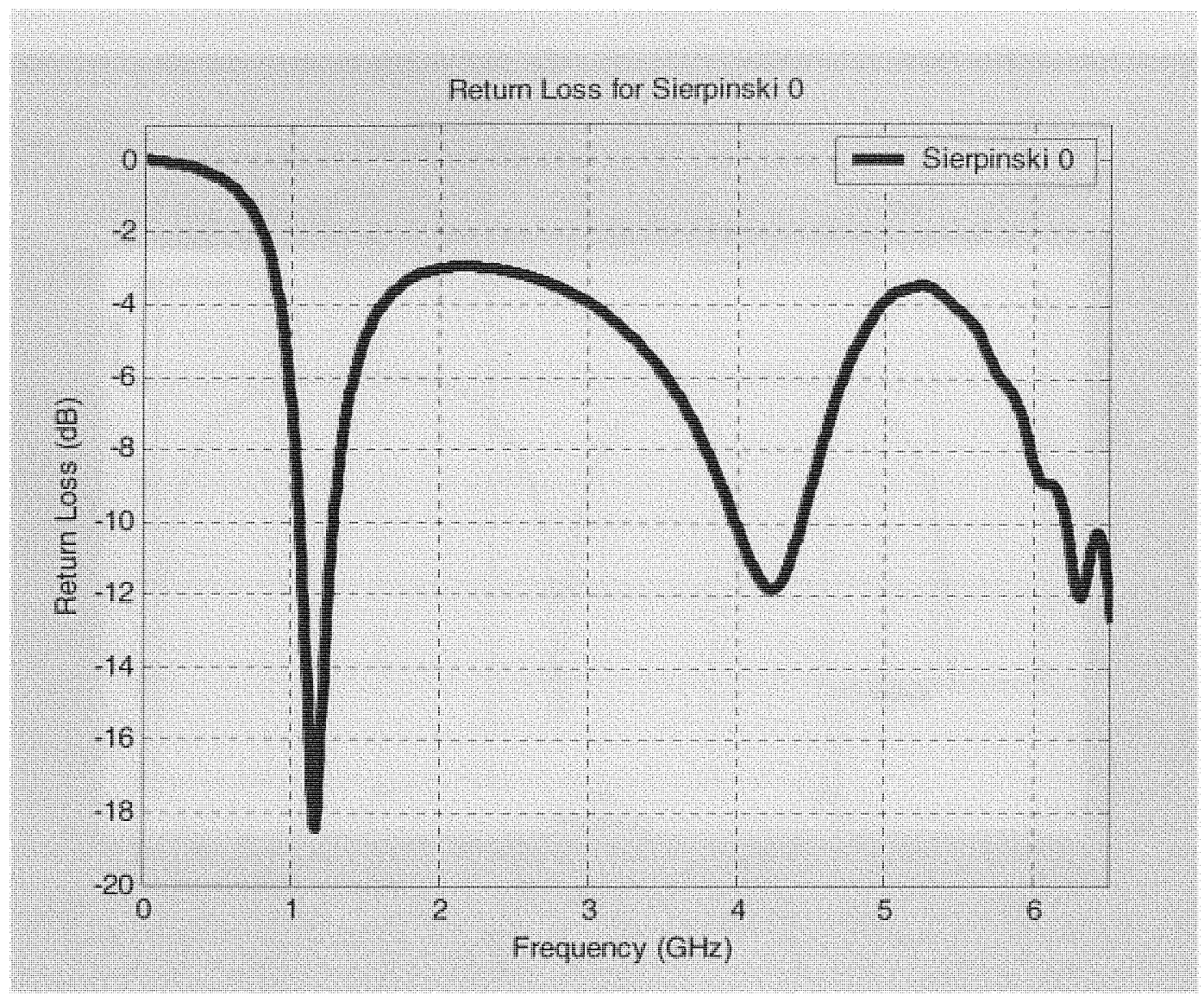

Figure 74 Return Loss for Sierpinski 0

The figure above shows the return loss for the sierpinski 0 fractal. The results where plot using Matlab software. This was used to validate the results obtained from the FDTD simulation. The impedance bandwidth obtained from the numerical calculations of the Sierpinski 0 fractal has an operational bandwidth ranges from $1.23 \mathrm{GHz}$ to $4.3 \mathrm{GHz}$. It was found that the S11 response for the Sierpinski 0 was $-19 \mathrm{~dB}$. 


\subsection{XFDTD Gain Plot of Sierpinski 0}

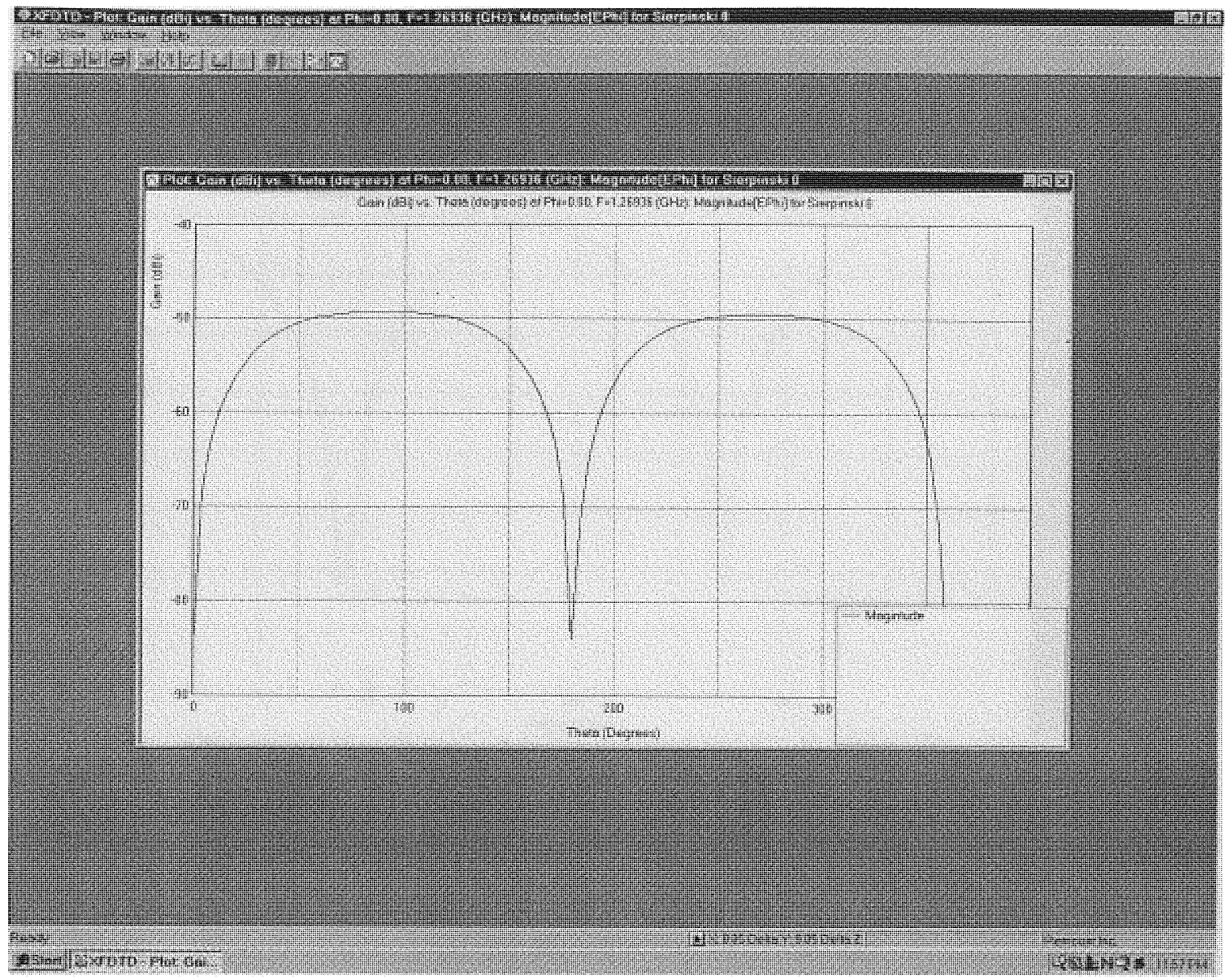

Figure 75 FDTD Simulated Gain of Sierpinski 0

The figure above shows the Gain vs Angle XFDTD simulation. 


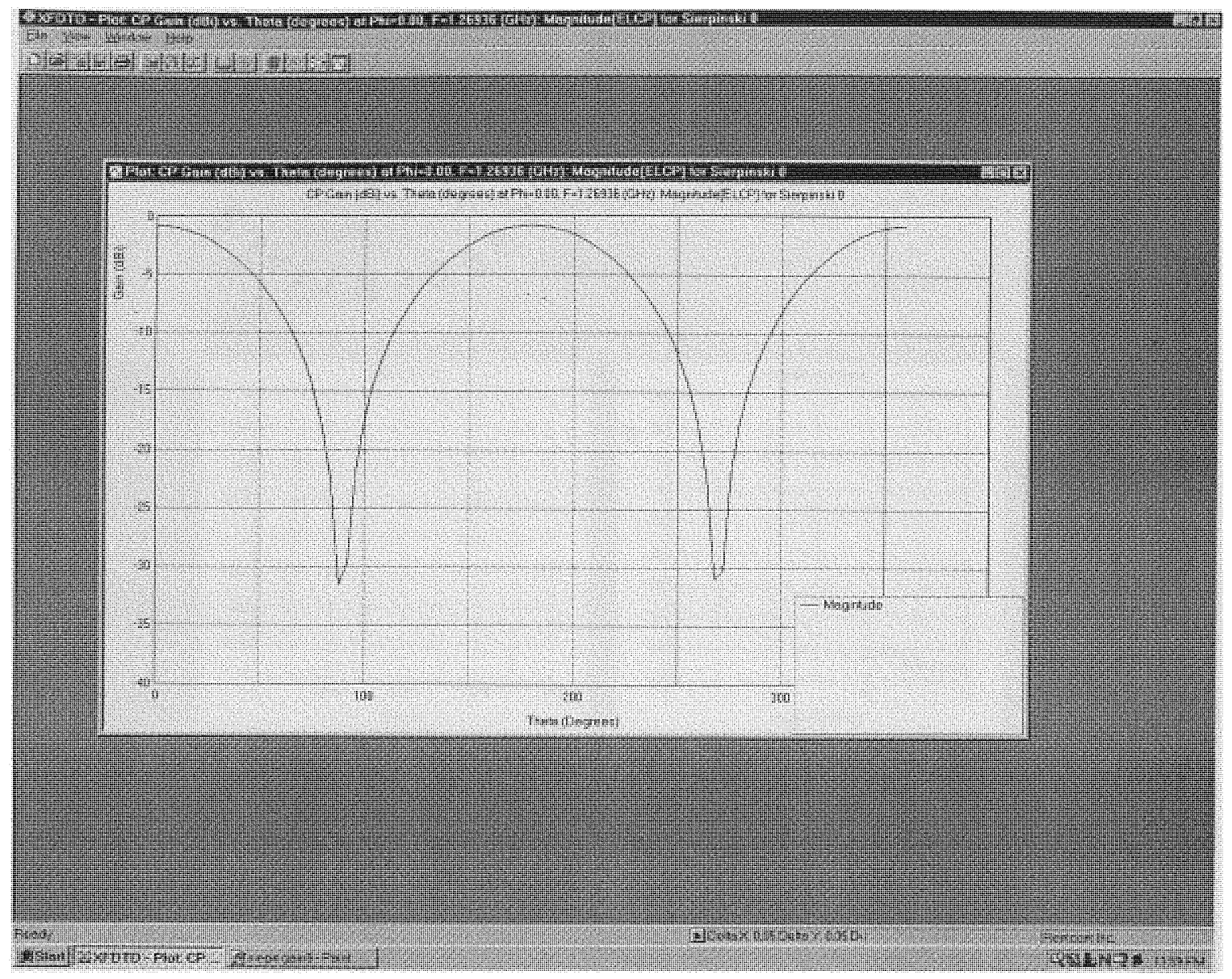

Figure 76 FDTD Simulated Constant Phi Gain of Sierpinski 0

The figure above shows the Gain vs Angle XFDTD simulation at Constant Phi. 


\subsection{XFDTD Run Parameters for Sierpinski 1}

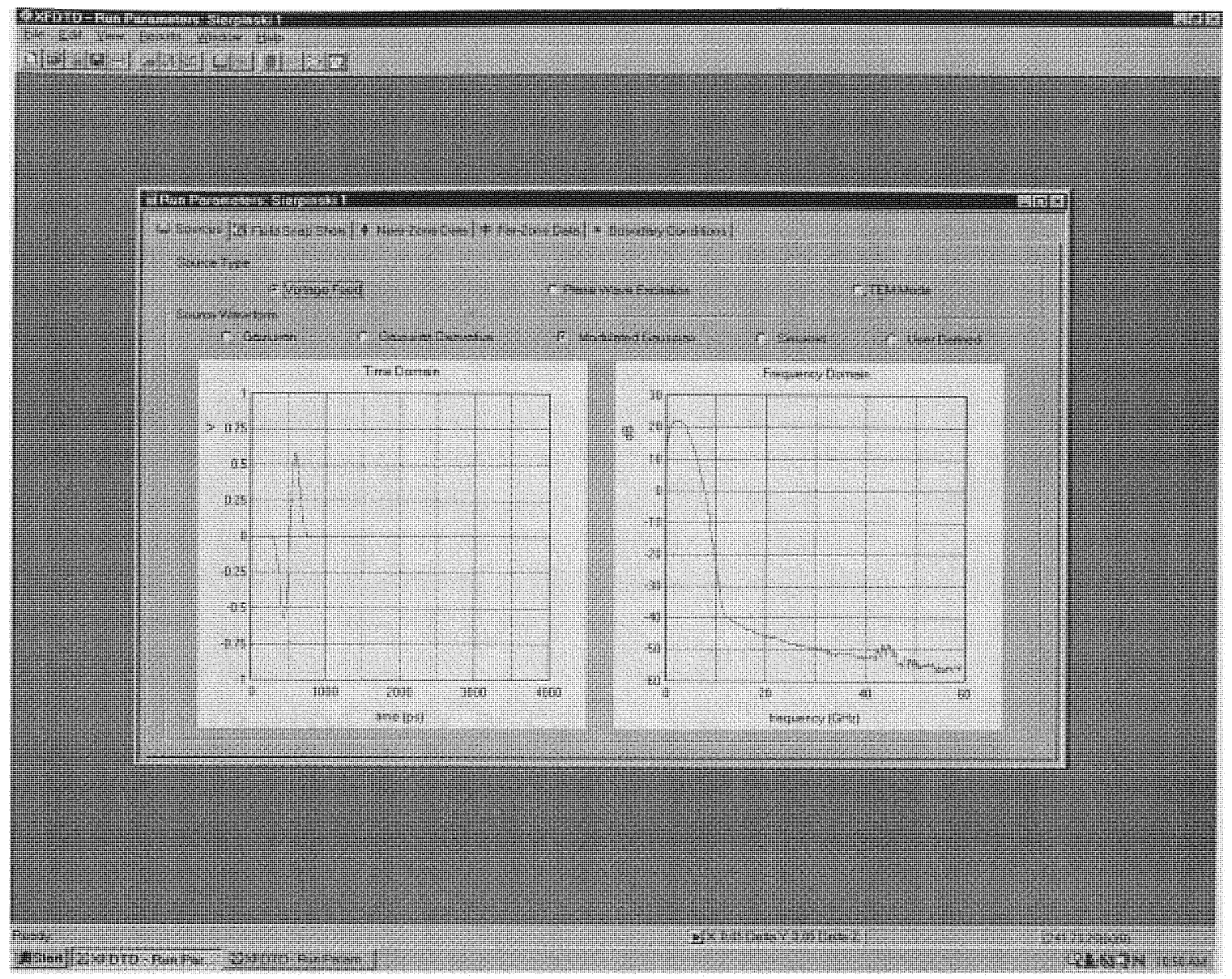

Figure 77 FDTD Run Parameter for Sierpinski 1

Figure 77 shows the setup run parameters for a FDTD simulation. The Modulated Gaussian was implemented in the setup. 


\subsection{Sierpinski 1 in XFDTD Space}

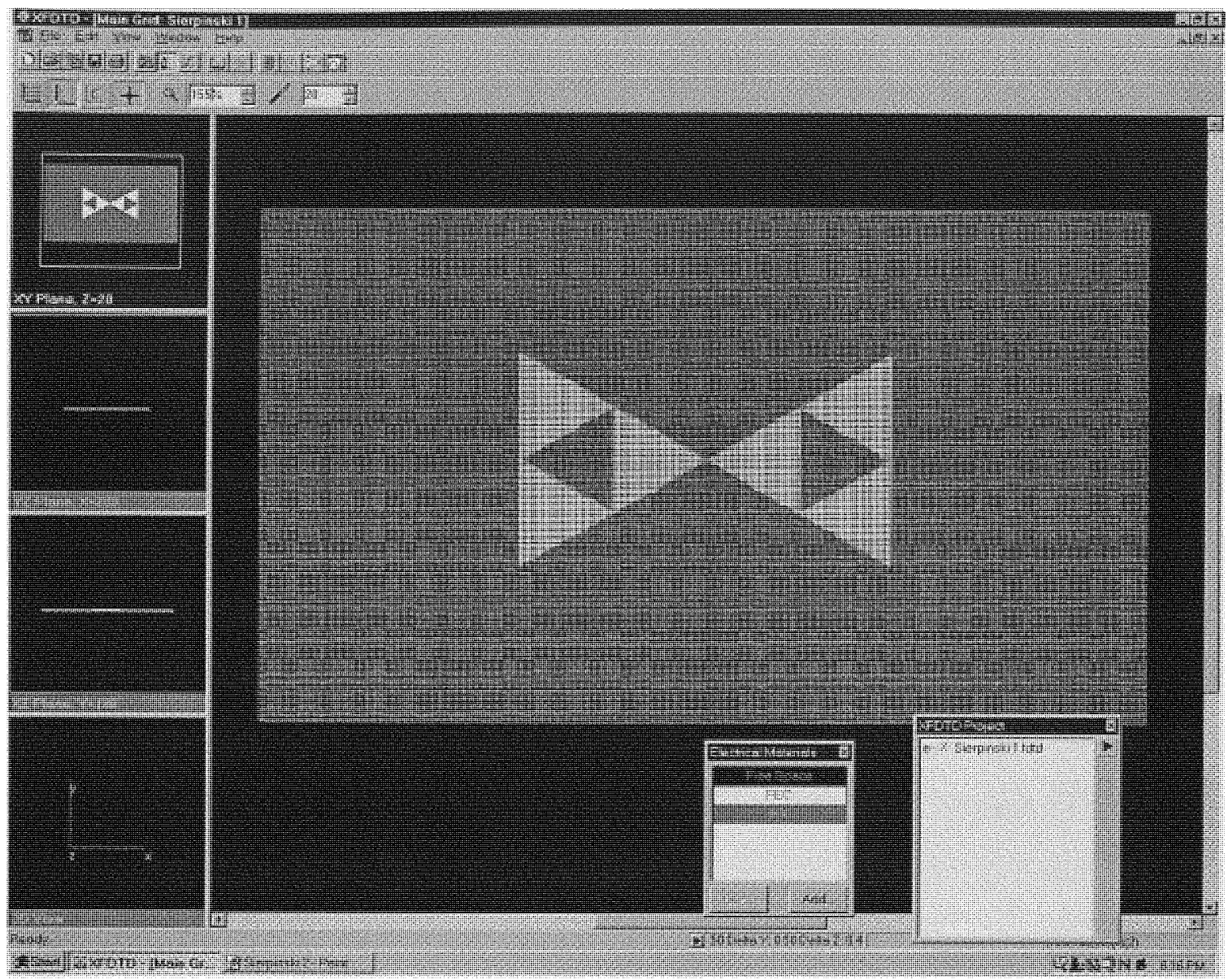

Figure 78 Sierpinski 1 at XY Plane in XFDTD Space 


\subsection{XFDTD Input Impedance Plot of Sierpinski 1}

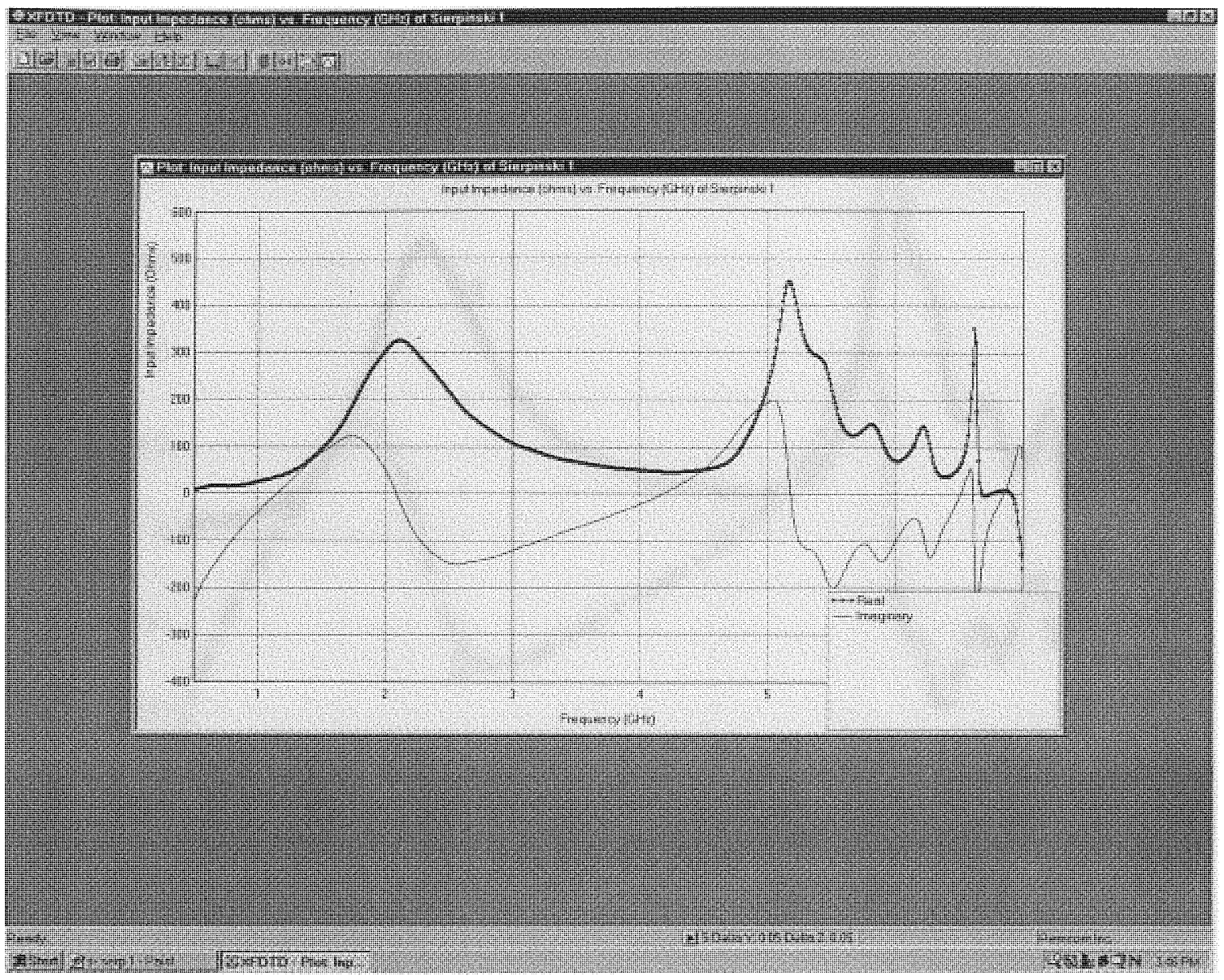

Figure 79 Input Impedance of Sierpinski 1

Figure 79 shows the Imaginary and Real part of the input impedance of Sierpinski

1 fractal. This result was obtained from the FDTD simulation. 
Figure 80 below shows the Imaginary and Real part of the input impedance of Sierpinski 1 fractal.

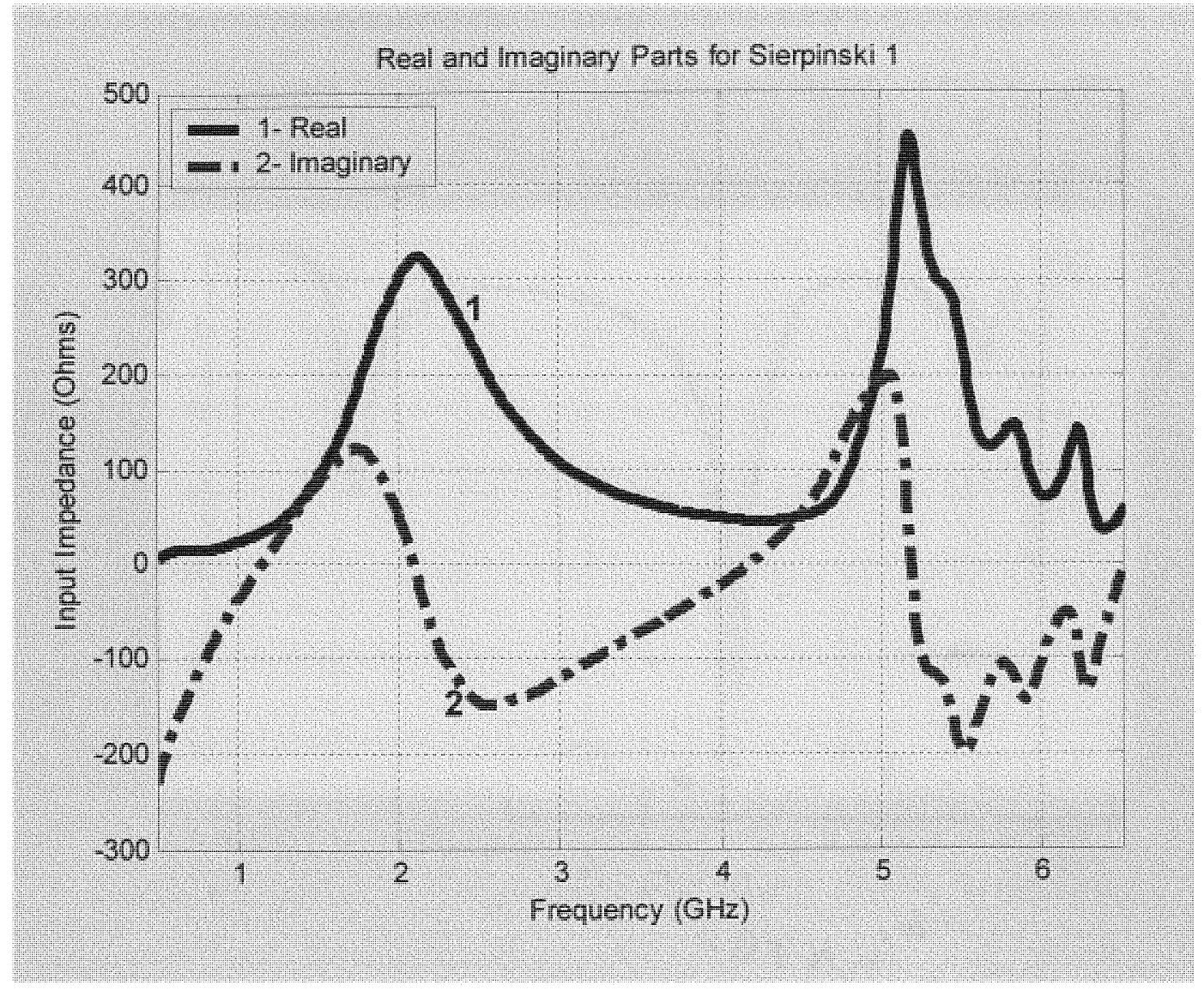

Figure 80 Real and Imaginary Input Impedance of Sierpinski 1 


\subsection{XFDTD $S_{11}$ Parameter Plot of Sierpinski 1}

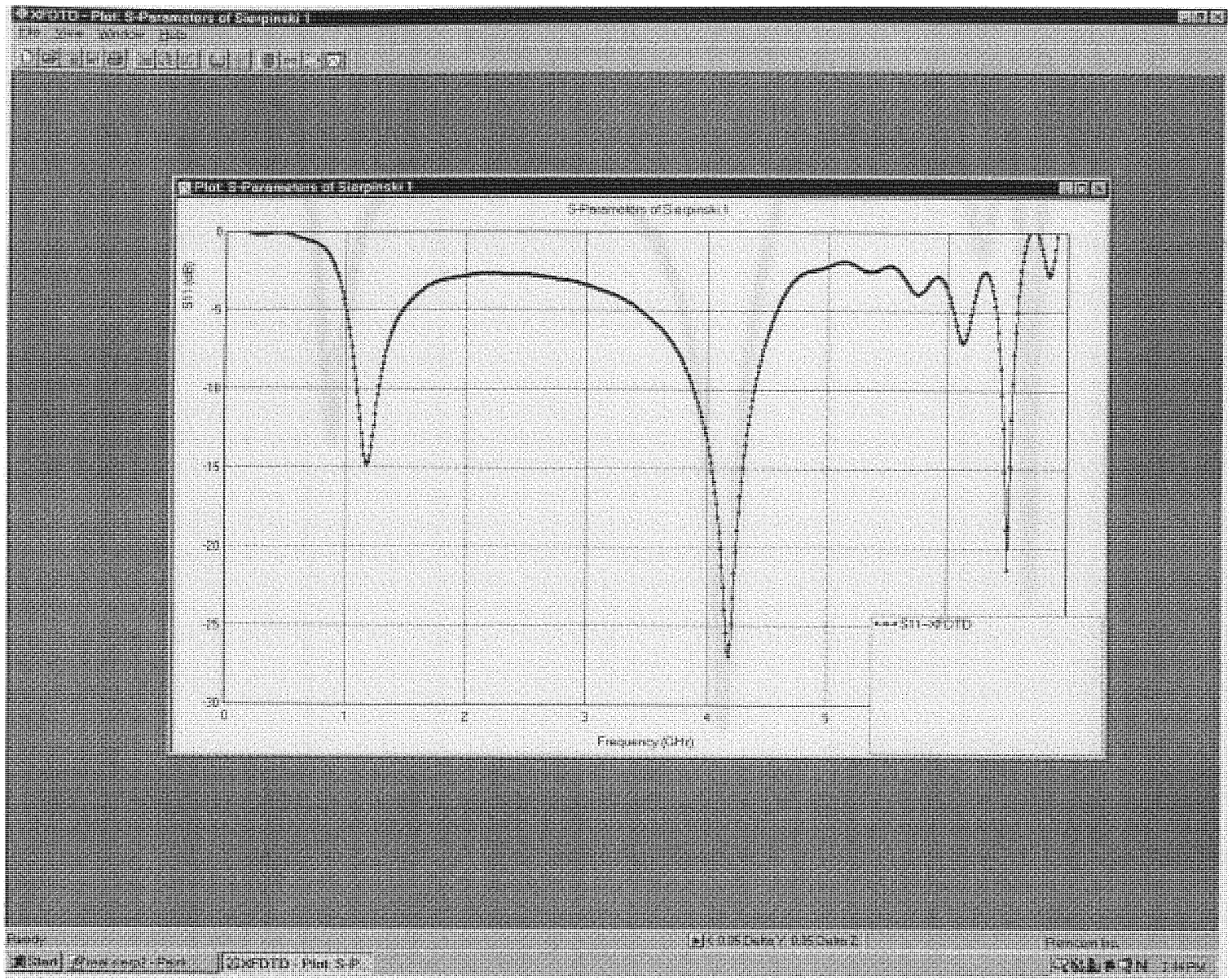

Figure $81 \mathrm{~S}_{11}$ parameter of Sierpinski 1 


\subsection{Return Loss for Sierpinski 1}

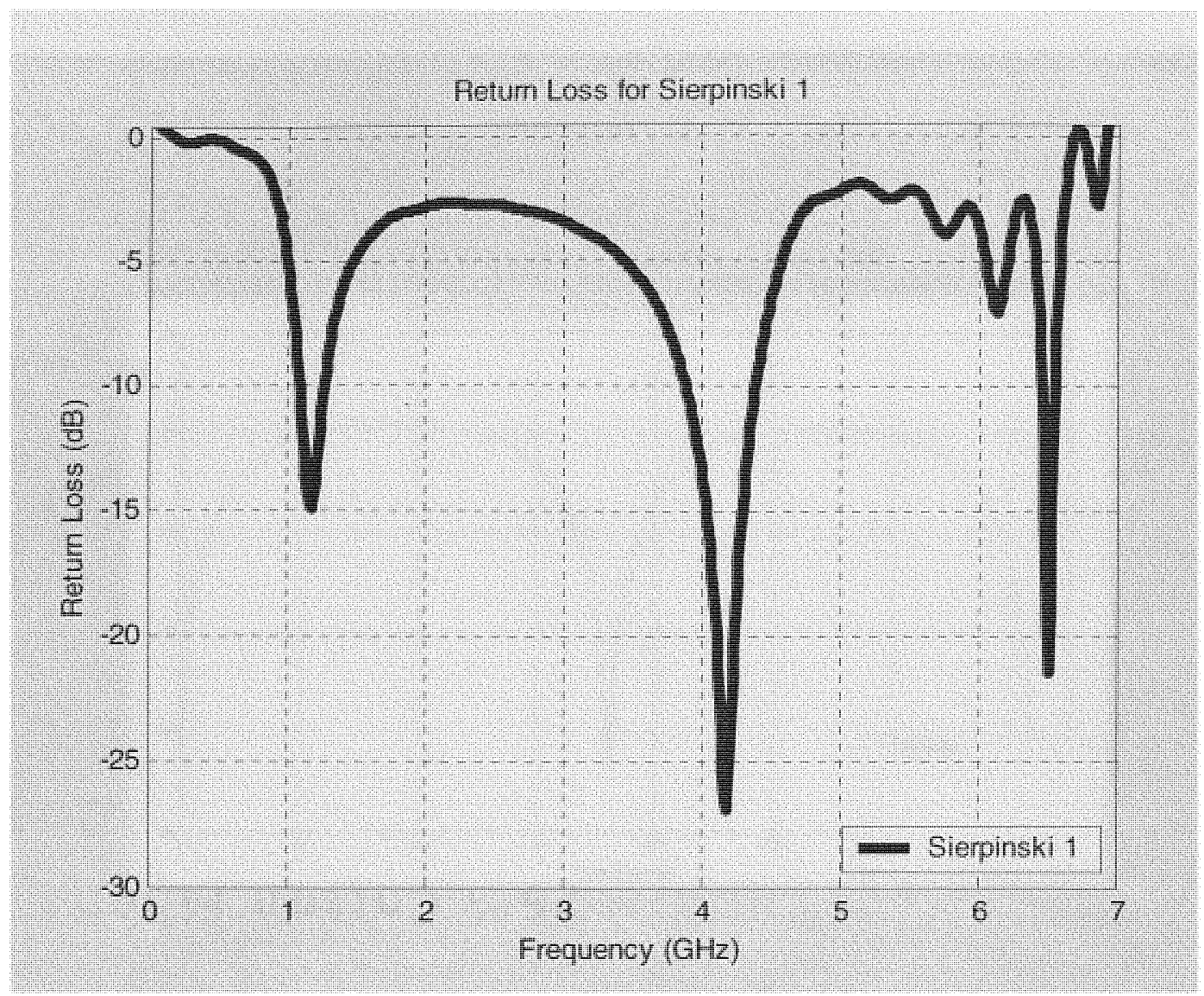

Figure 82 Return Loss of Sierpinski 1 fractal

Figure 82 above shows the return loss for the sierpinski 1 fractal. The results where plot using Matlab software. This was used to validate the results obtained from the FDTD simulation. The impedance bandwidth obtained from the numerical calculations of the Sierpinski 1 fractal has an operational bandwidth ranges from $1.25 \mathrm{GHz}$ to $4.3 \mathrm{GHz}$ and $6.8 \mathrm{GHz}$. It was found that the $\mathrm{S} 11$ response for the Sierpinski 1 at resonant frequencies of $1.25 \mathrm{GHz}$ was $-15 \mathrm{~dB}, 4.3 \mathrm{GHz}$ was $-27 \mathrm{~dB}$ and $6.8 \mathrm{GHz}$ was $-22 \mathrm{~dB}$. 


\subsection{XFDTD Gain plot of Sierpinski 1}

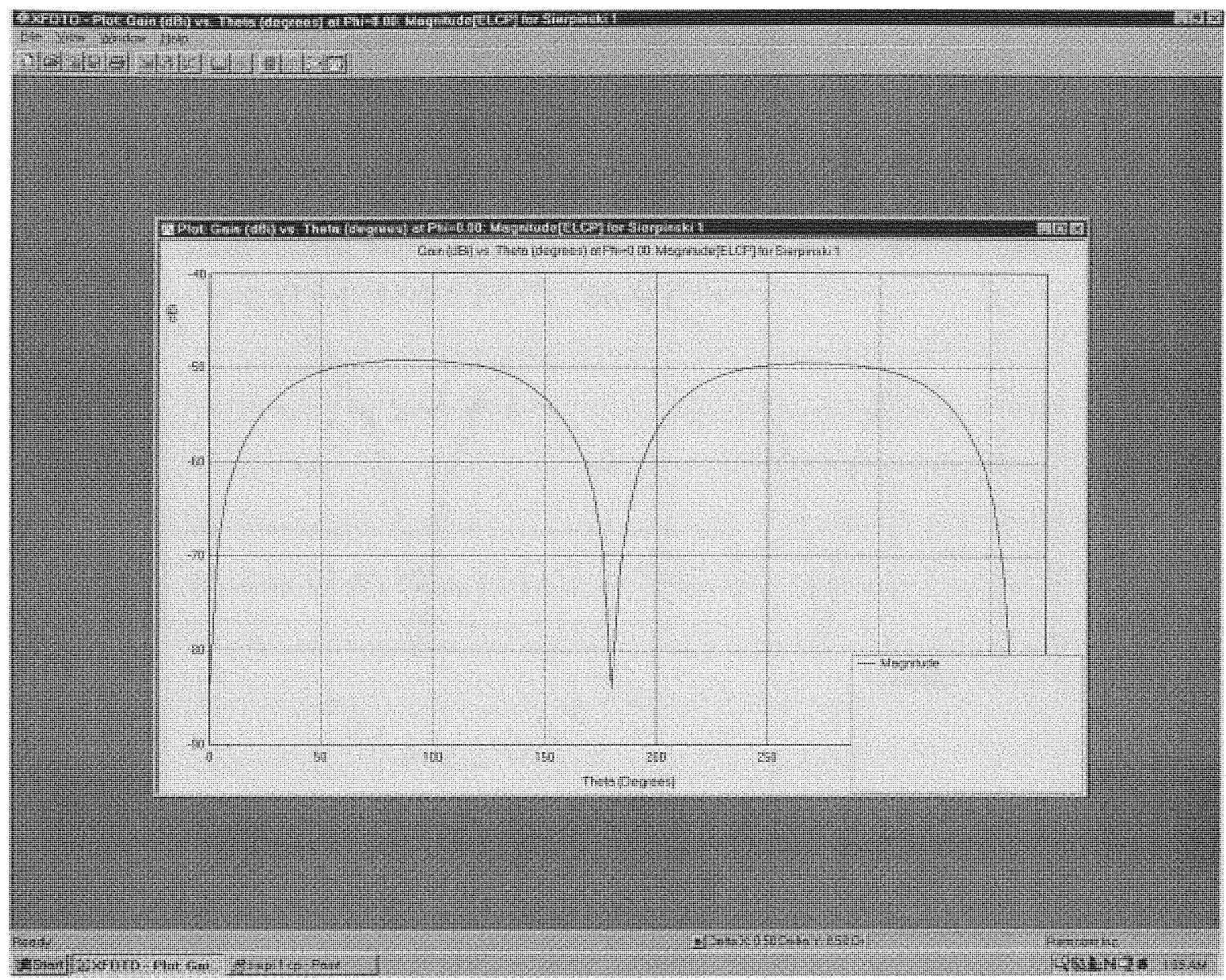

Figure 83 FDTD Simulated Gain of Sierpinski 1

The above figure 83 shows the Gain vs Angle XFDTD simulation. 


\subsection{XFDTD CP Gain Plot of Sierpinski 1}

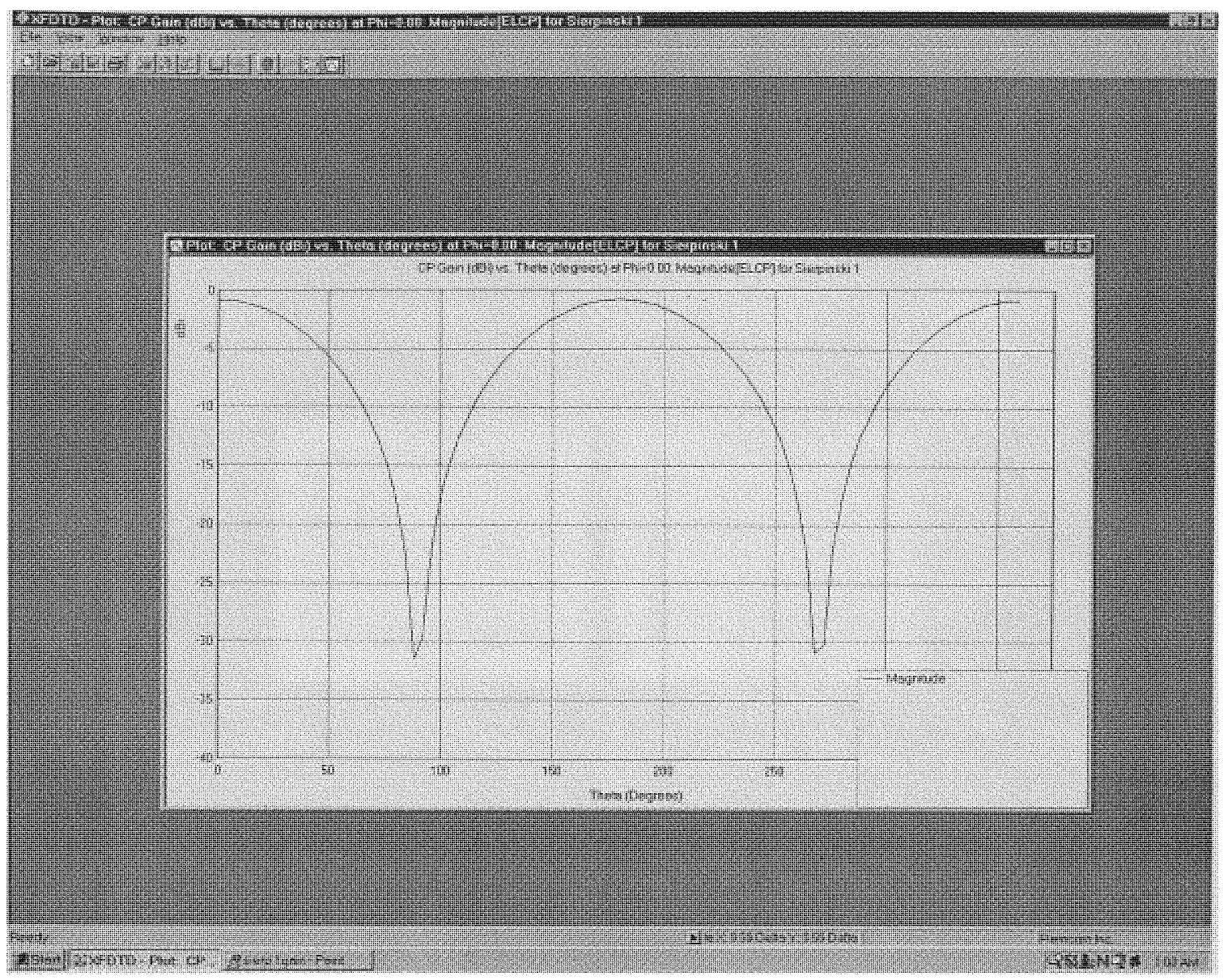

Figure 84 FDTD Simulated CP Gain of Sierpinski 1

The above figure shows the Gain vs Angle XFDTD simulation at Constant Phi. 


\subsection{XFDTD Run Parameters of Sierpinski 2}

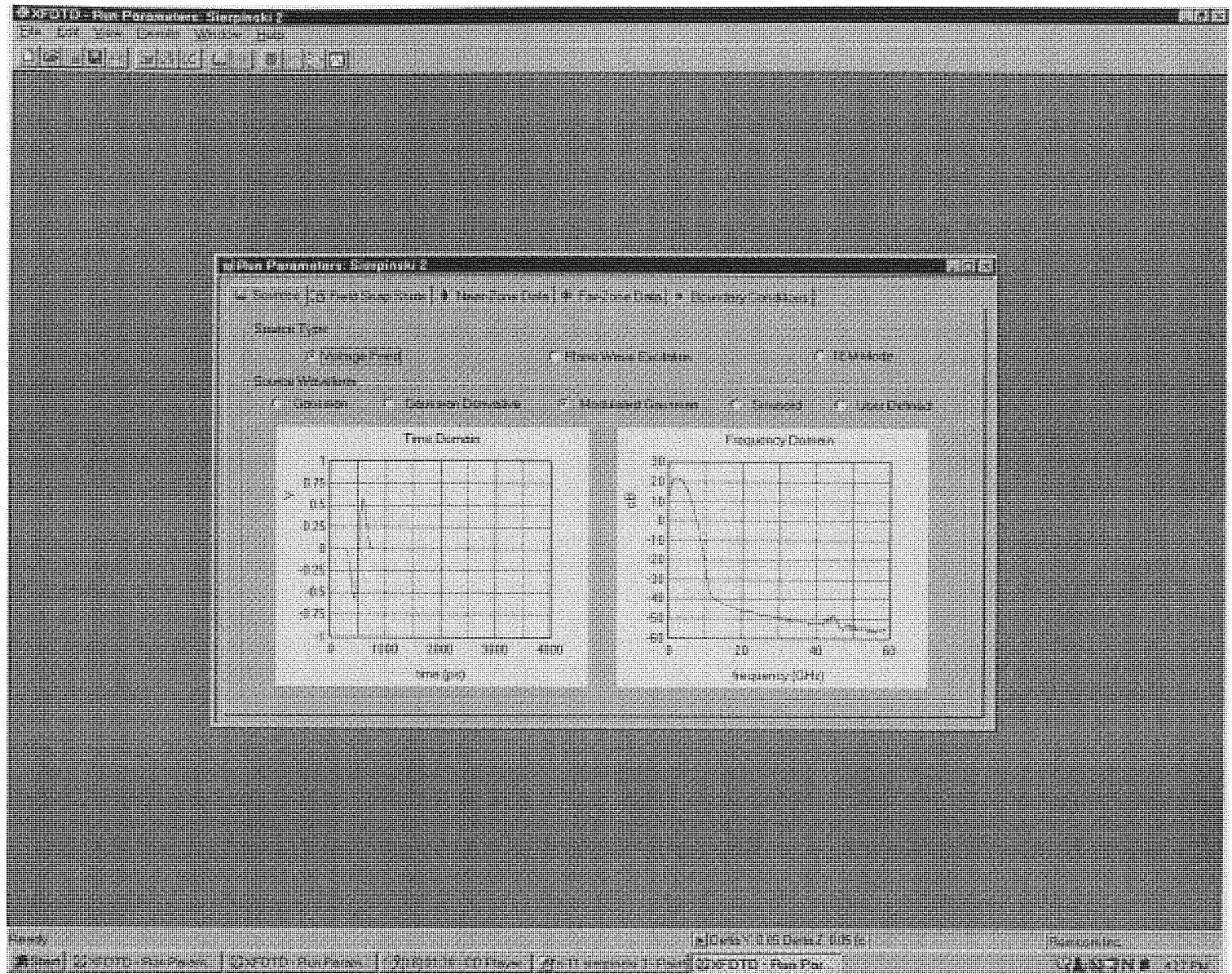

Figure 85 FDTD Run parameters for Sierpinski 2

Figure 85 shows the setup run parameters for a FDTD simulation. The Modulated Gaussian was implemented in the setup. 


\subsection{Sierpinski 2 in XFDTD Space}

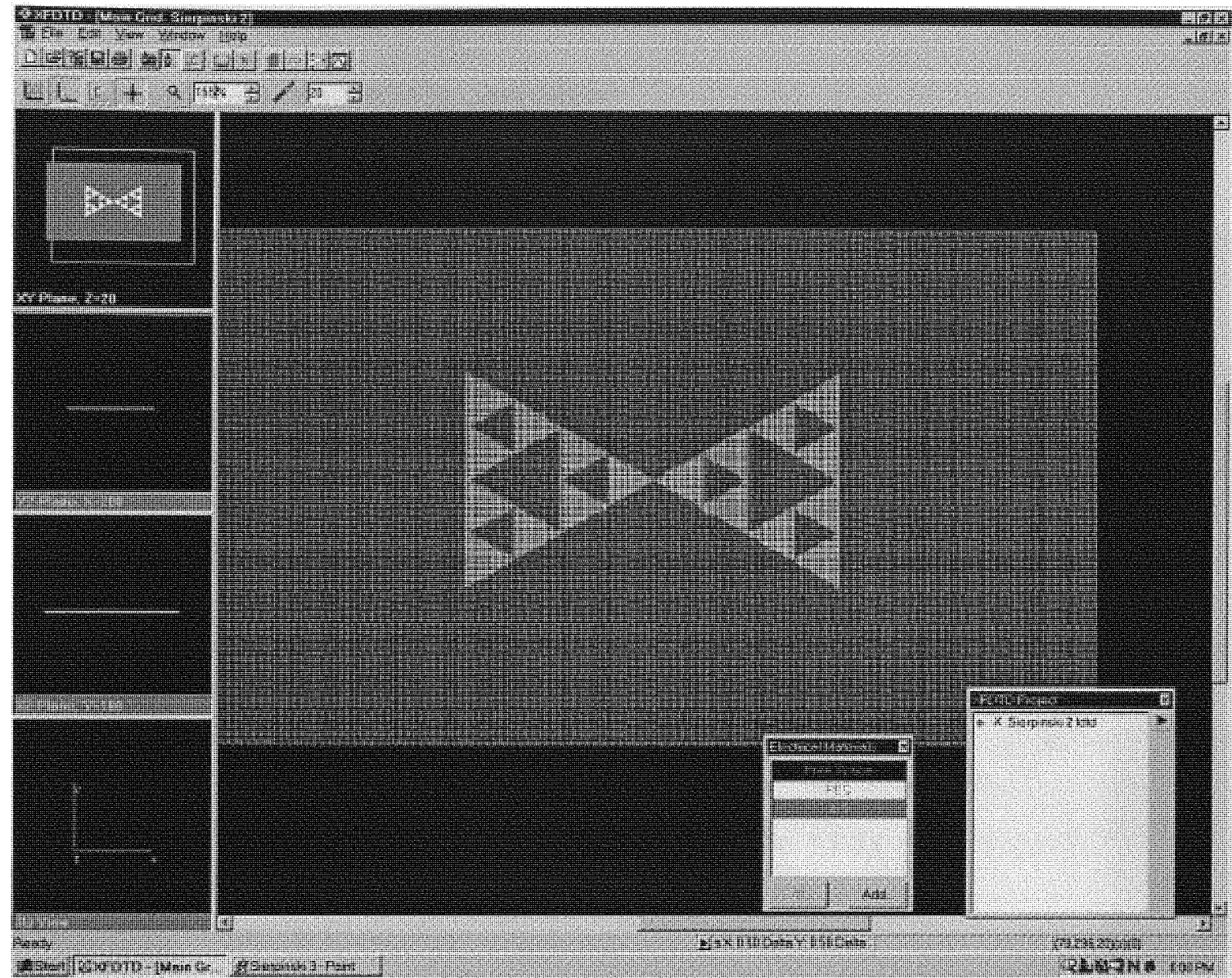

Figure 86 Sierpinski 2 at XY Plane in XFDTD Space 


\subsection{XFDTD Input Impedance plot of Sierpinski 2}

Figure 87 below shows the Imaginary and Real parts of the input impedance. The results were obtained from the XFDTD simulation.

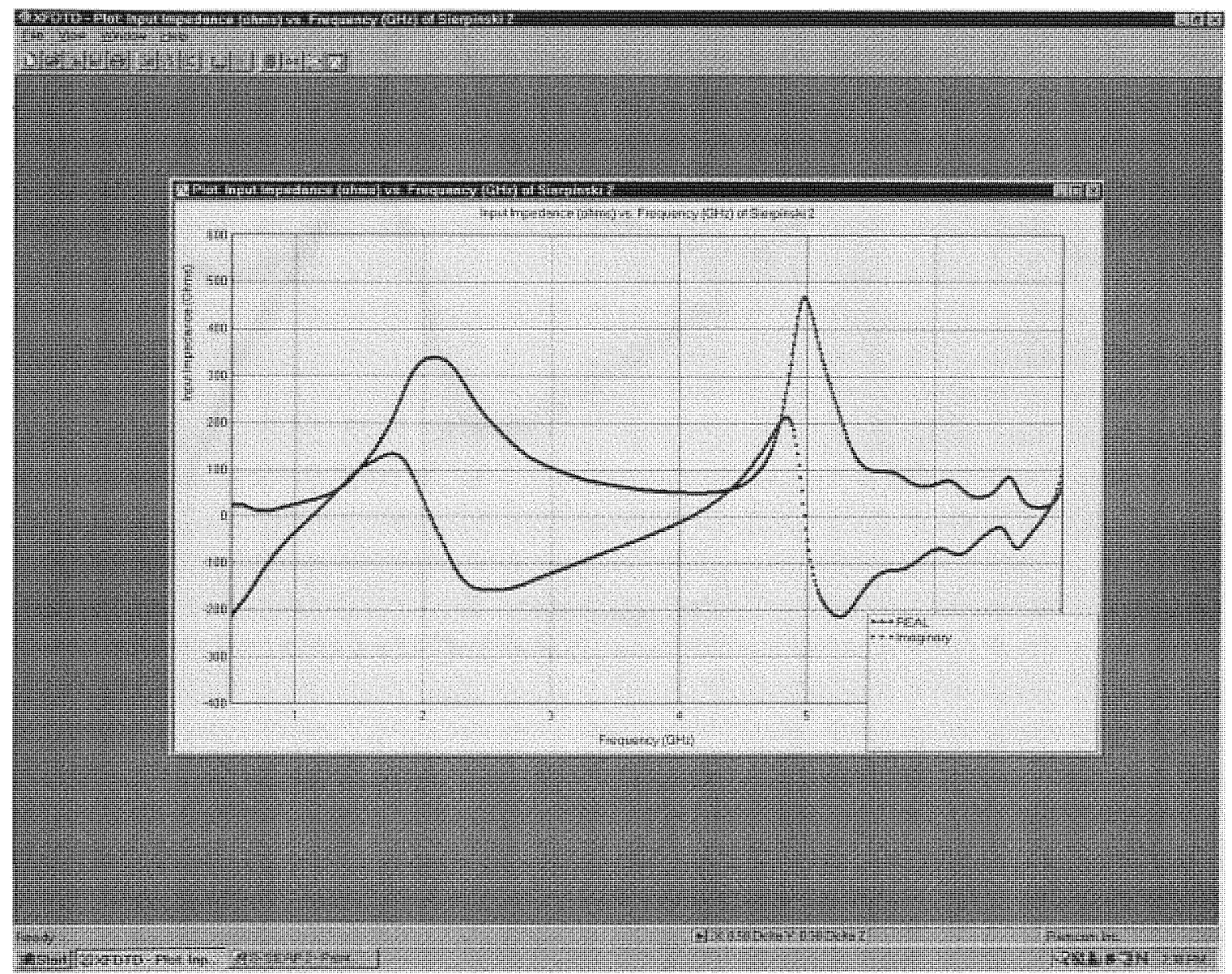

Figure 87 Real and Imaginary Input Impedance of Sierpinski 2 
Figure 88 below shows the Imaginary and Real parts of the input impedance

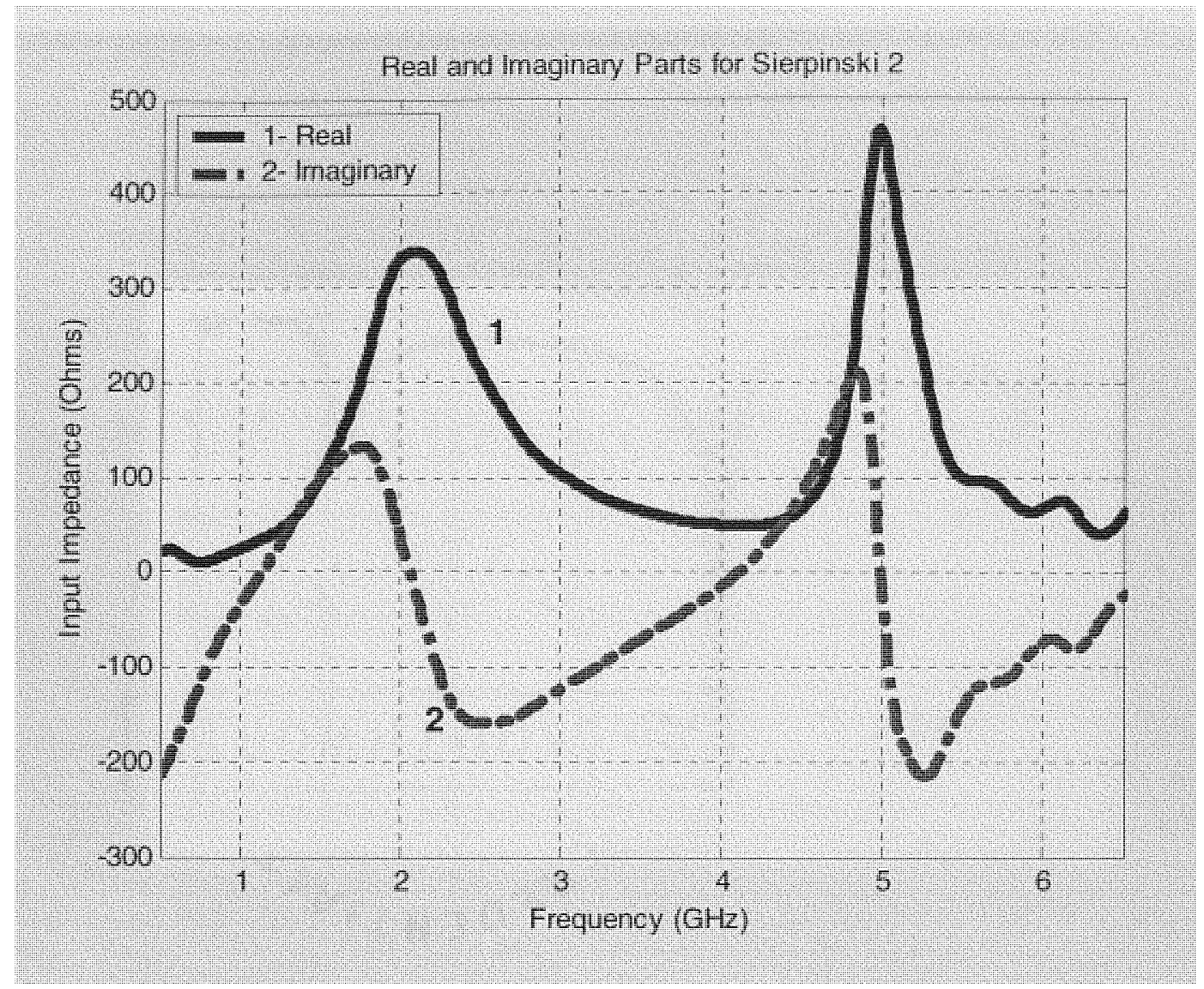

Figure 88 Real and Imaginary Input Impedance of Sierpinski 2 


\subsection{XFDTD $S_{11}$ Parameter plot of Sierpinski 2}

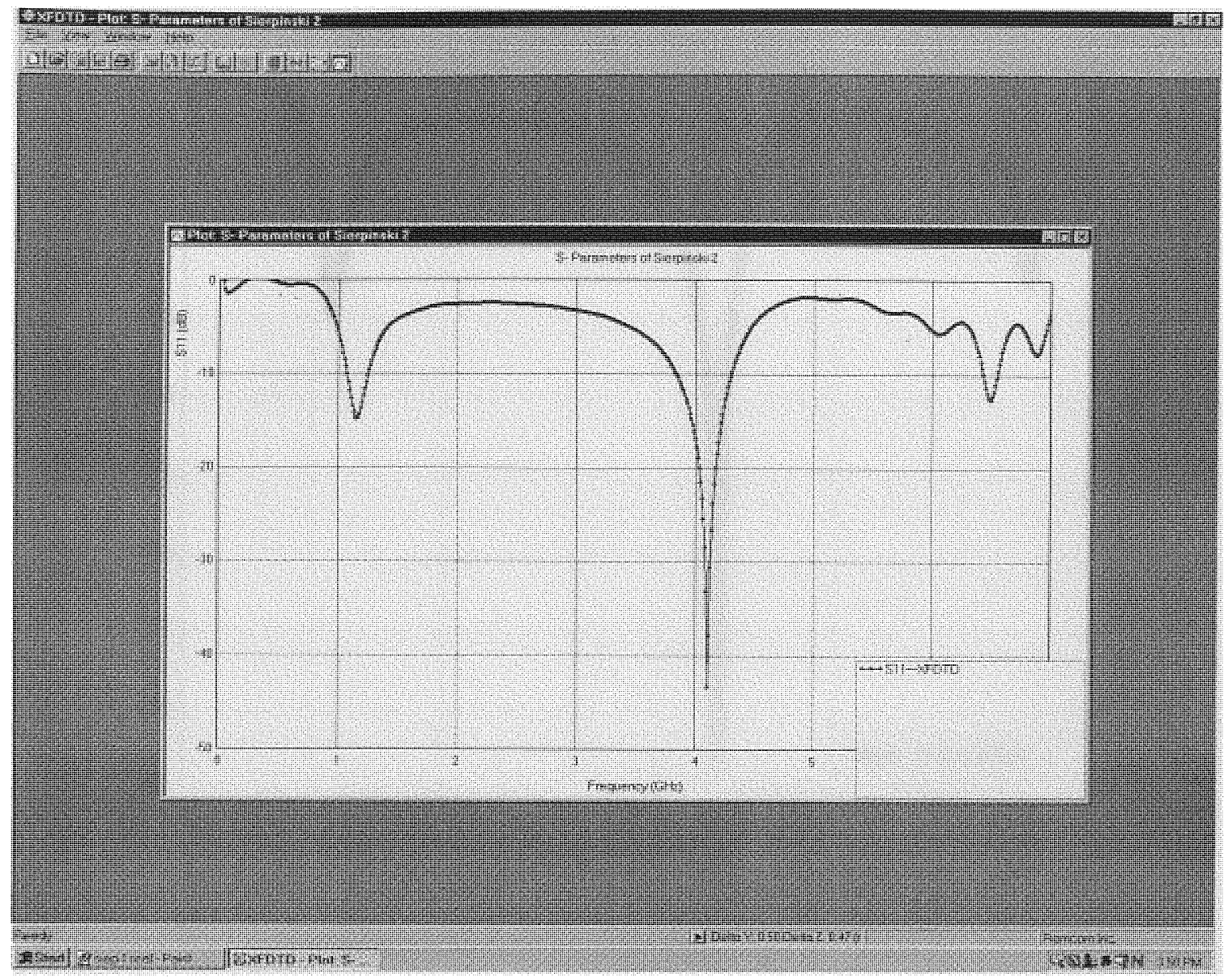

Figure $89 S_{11}$ parameter of Sierpinski 2 


\subsection{Return Loss of Sierpinski 2}

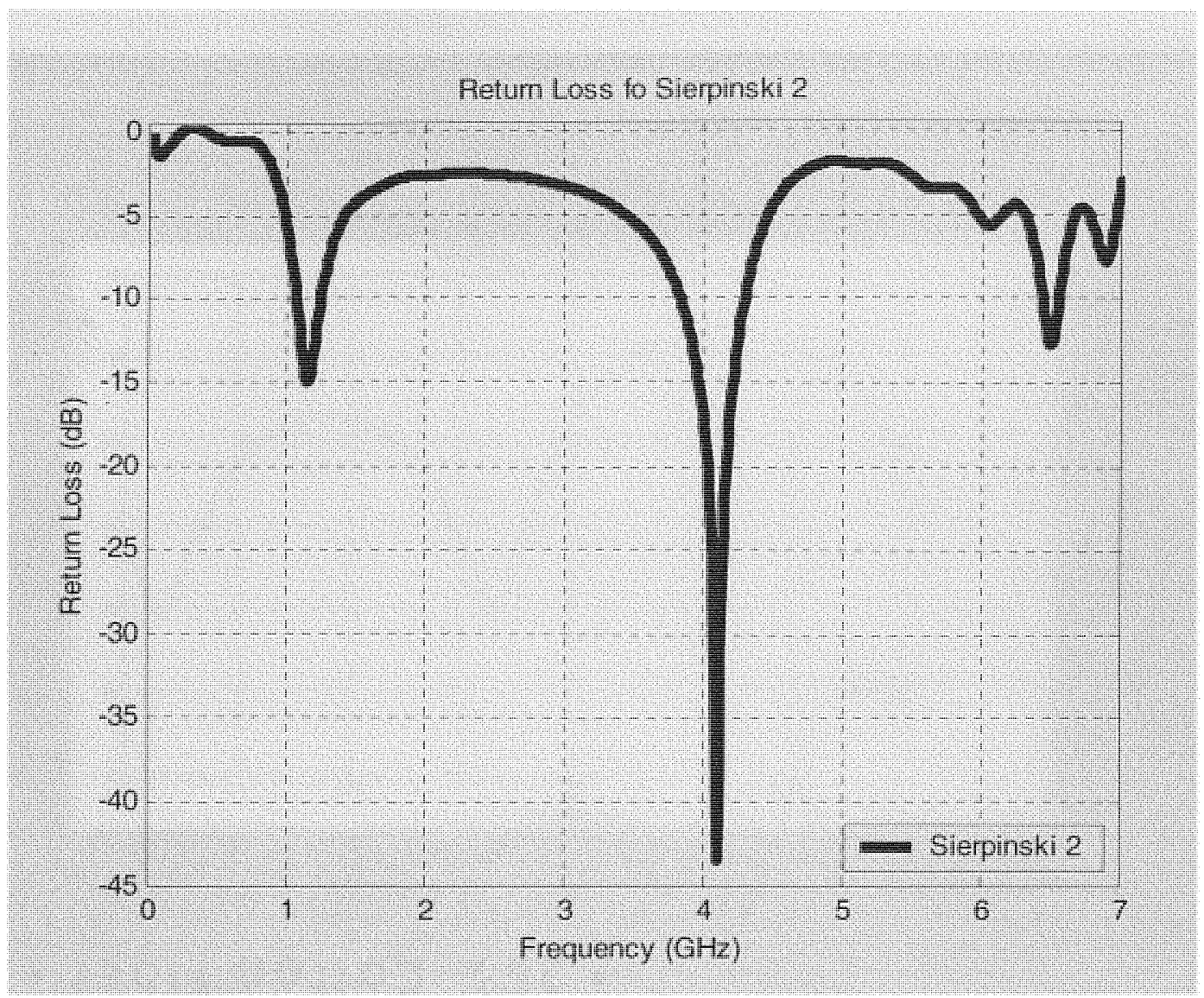

Figure 90 Return Loss of Sierpinski 2 Fractal

The figure 90 above shows the return loss for the sierpinski 2 fractal. The results where plot using Matlab software. This was used to validate the results obtained from the FDTD simulation. The impedance bandwidth obtained from the numerical calculations of the Sierpinski 2 fractal has an operational bandwidth ranges from $1.25 \mathrm{GHz}$ to $4.2 \mathrm{GHz}$ and $6.8 \mathrm{GHz}$. It was found that the $\mathrm{S} 11$ response for the Sierpinski 2 at the $1^{\text {St }}$ resonant frequency of $1.25 \mathrm{GHz}$ was $-15 \mathrm{~dB}$, the $2^{\text {nd }}$ resonant frequency at $4.2 \mathrm{GHz}$ was $-44 \mathrm{~dB}$ and at $3^{\text {rd }}$ resonant frequency $6.8 \mathrm{GHz}$ was $-13 \mathrm{~dB}$. 


\subsection{XFDTD Gain plot for Sierpinski 2}

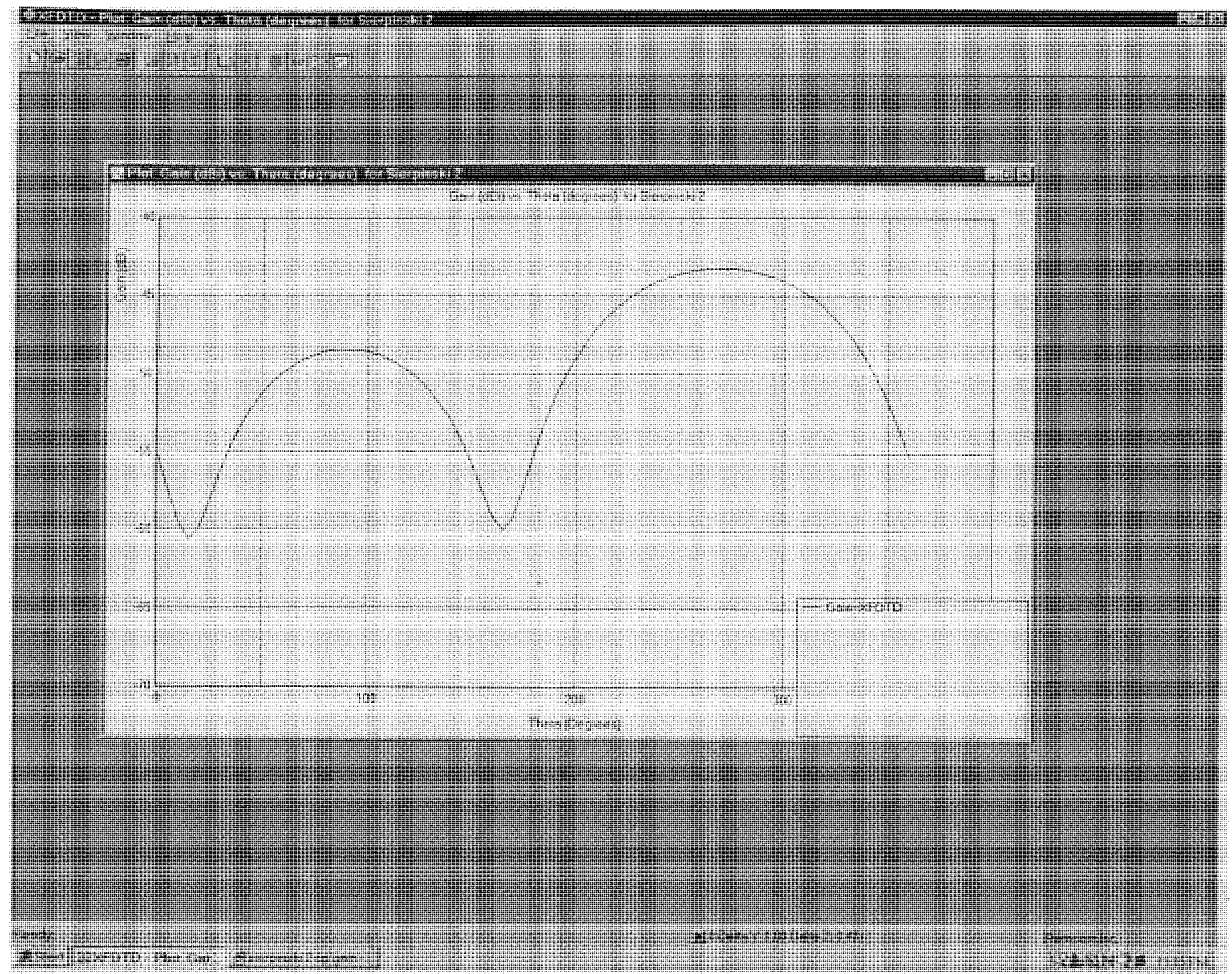

Figure 91 FDTD Simulated Gain of Sierpinski 2

The above figure shows the Gain vs Angle XFDTD simulation. 


\subsection{XFDTD CP Gain plot for Sierpinski 2}

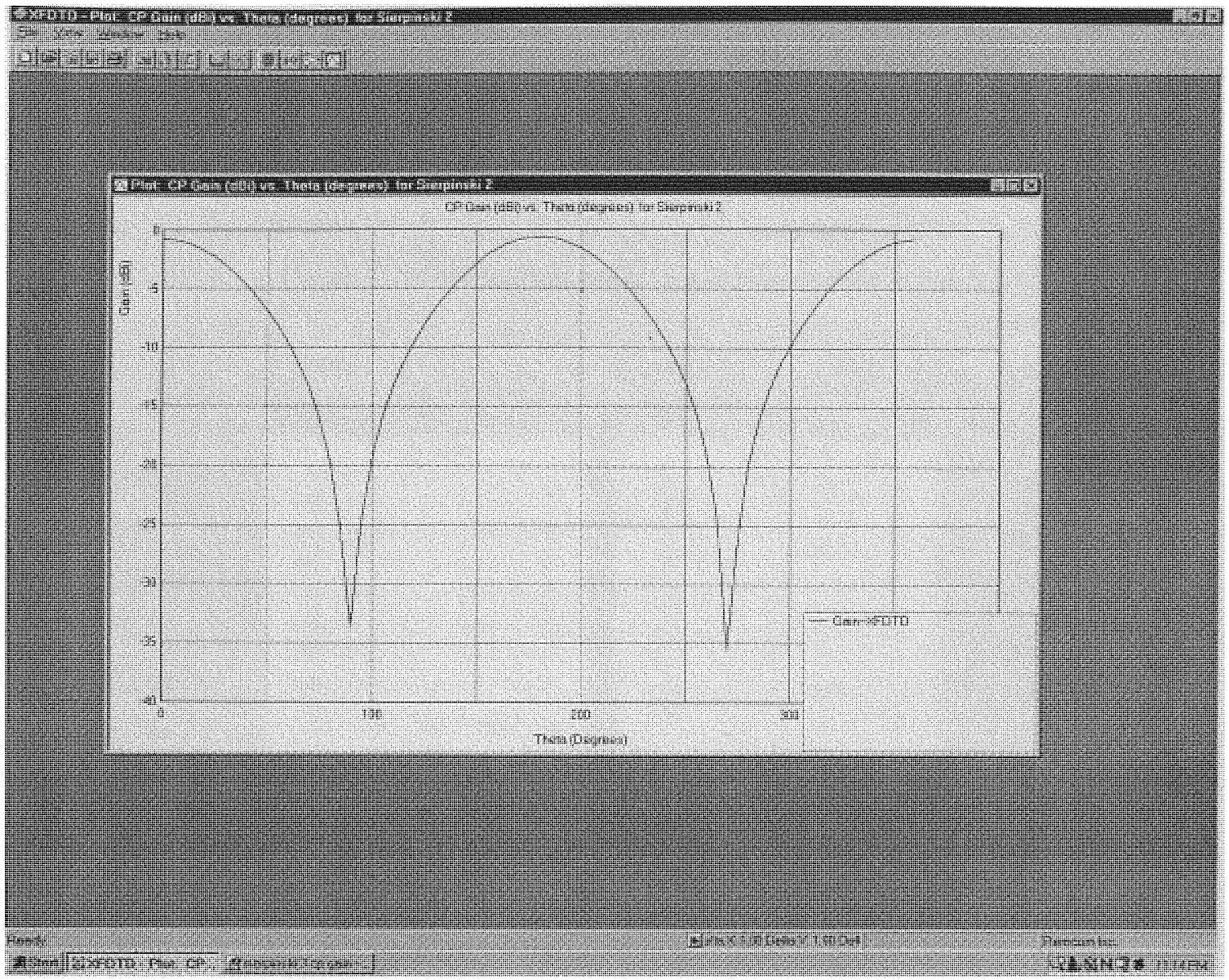

Figure 92 FDTD Simulated CP Gain of Sierpinski 2

The above figure shows the Gain vs Angle XFDTD simulation at Constant Phi. 


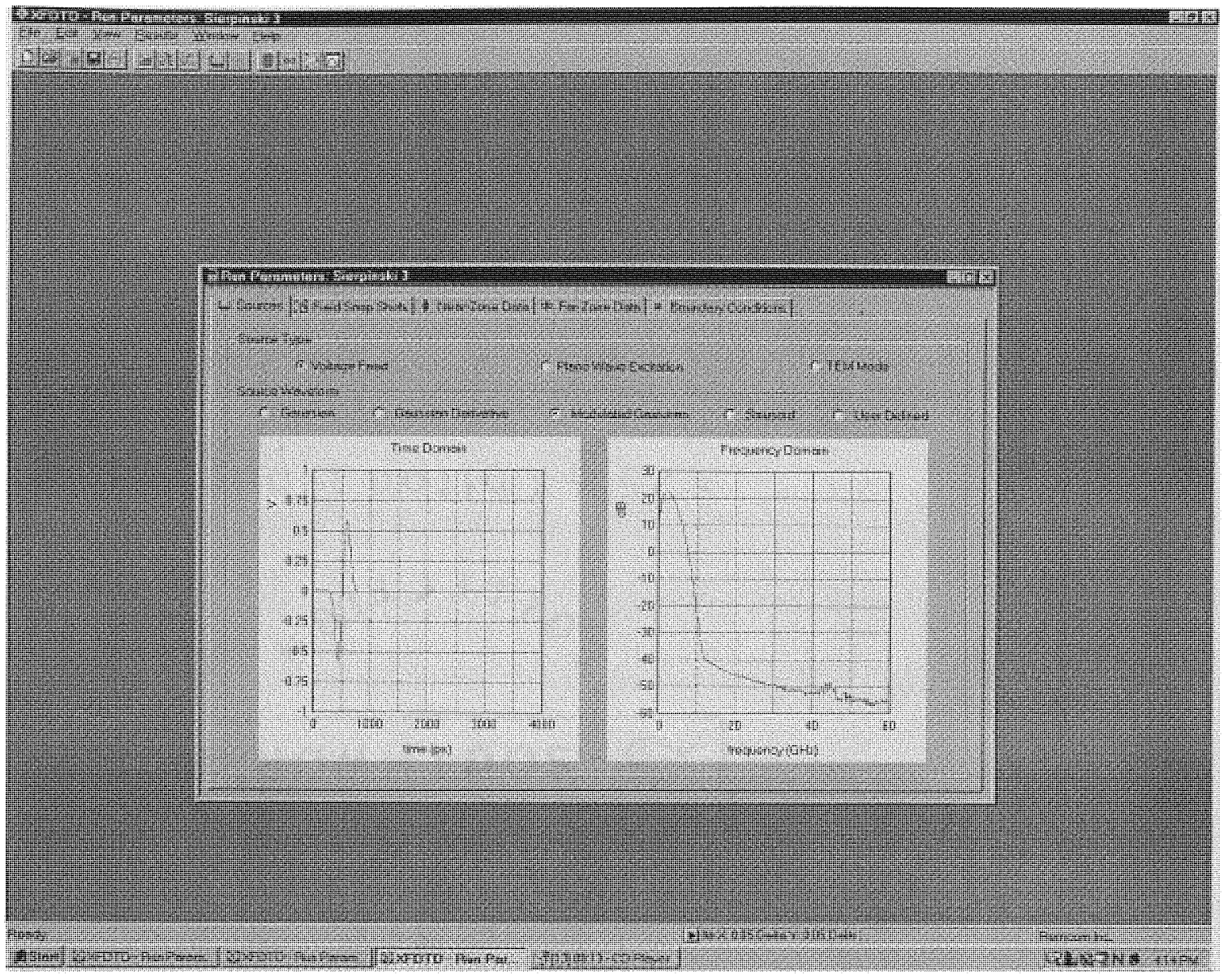

Figure 93 FDTD Run Parameters of Sierpinski 3

Figure 93 shows the setup run parameters for a FDTD simulation. The Modulated Gaussian was implemented in the setup. 


\subsection{Sierpinski 3 in XFDTD Space}

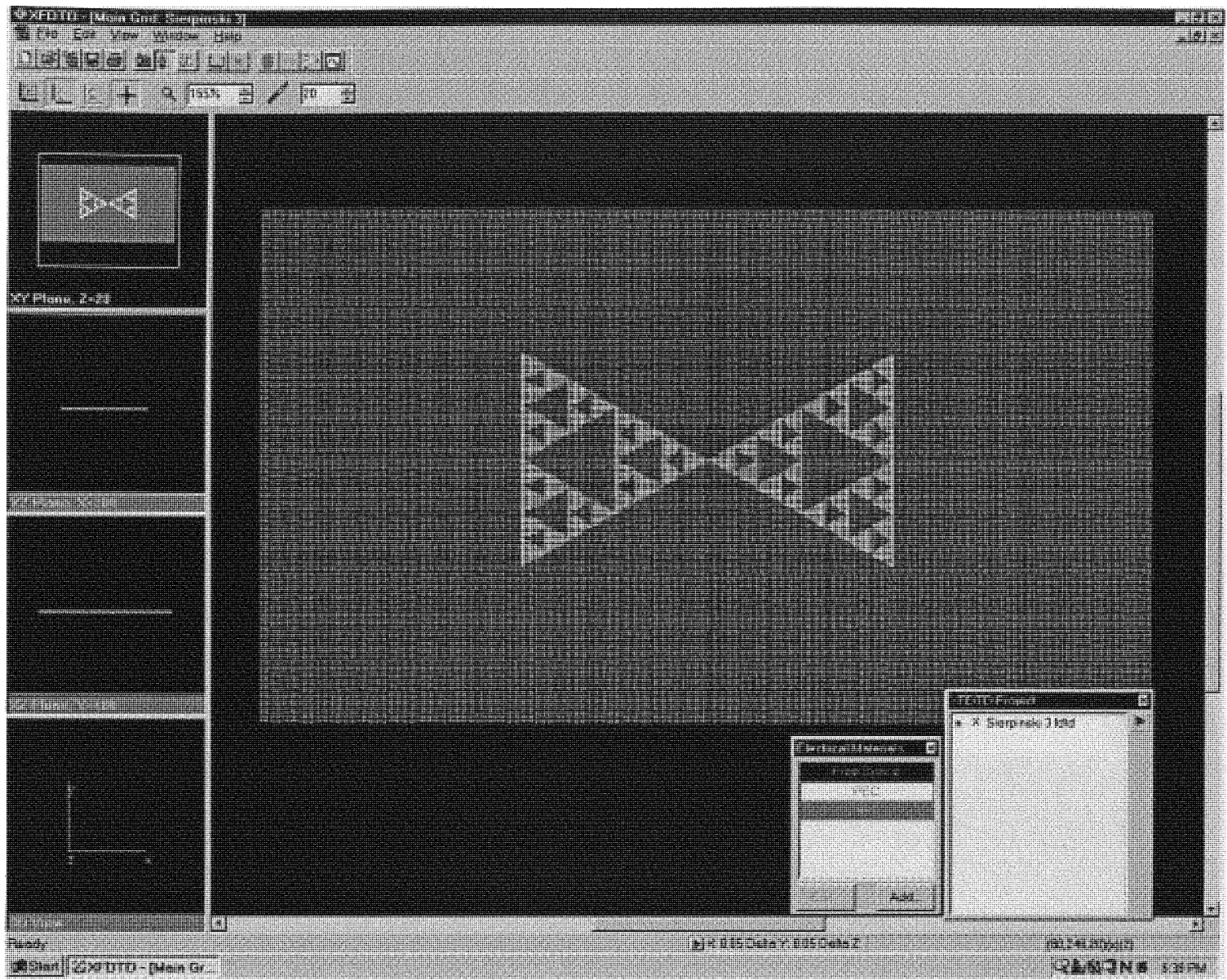

Figure 94 Sierpinski 3 in XFDTD Space 


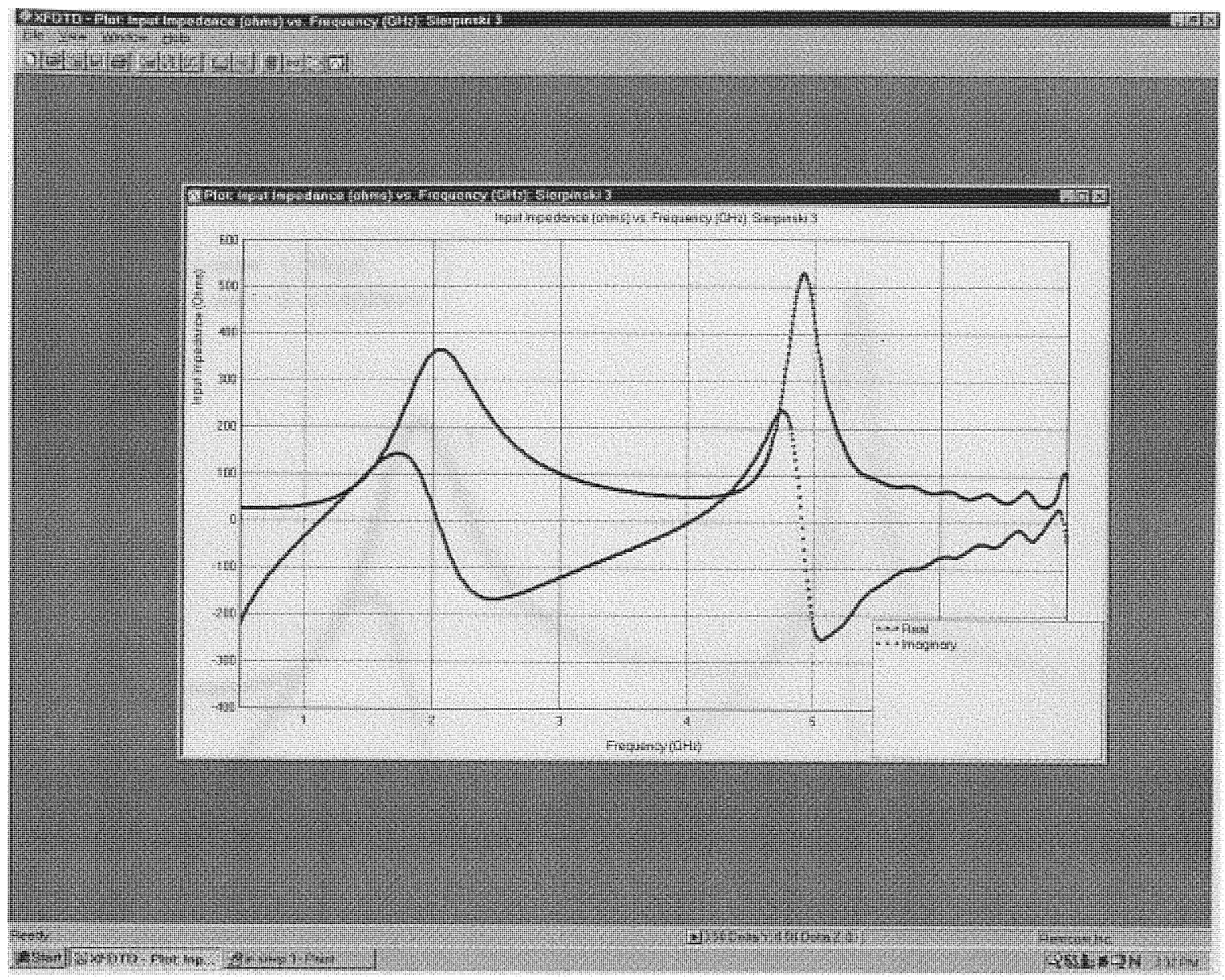

Figure 95 Input Impedance of Sierpinski 3

Figure 95 shows the Imaginary and Real part of the input impedance of Sierpinski 3 fractal. This was obtained from the FDTD simulation. 
Figure 96 below shows the Imaginary and Real part of the input impedance of Sierpinski 3 fractal.

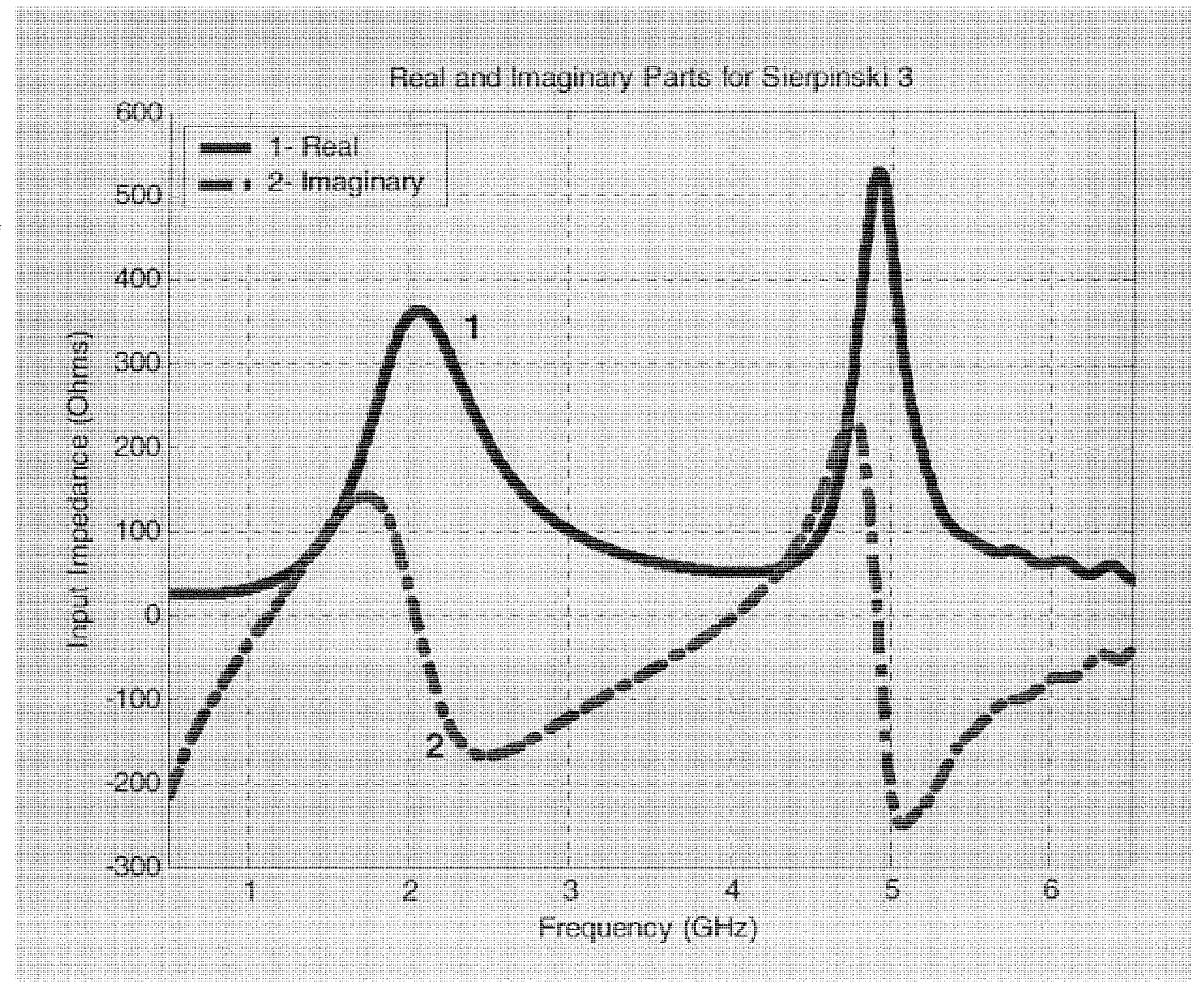

Figure 96 Real and Imaginary Input Impedance of Sierpinski 3 


\subsection{XFDTD $S_{11}$ parameter of Sierpinski 3}

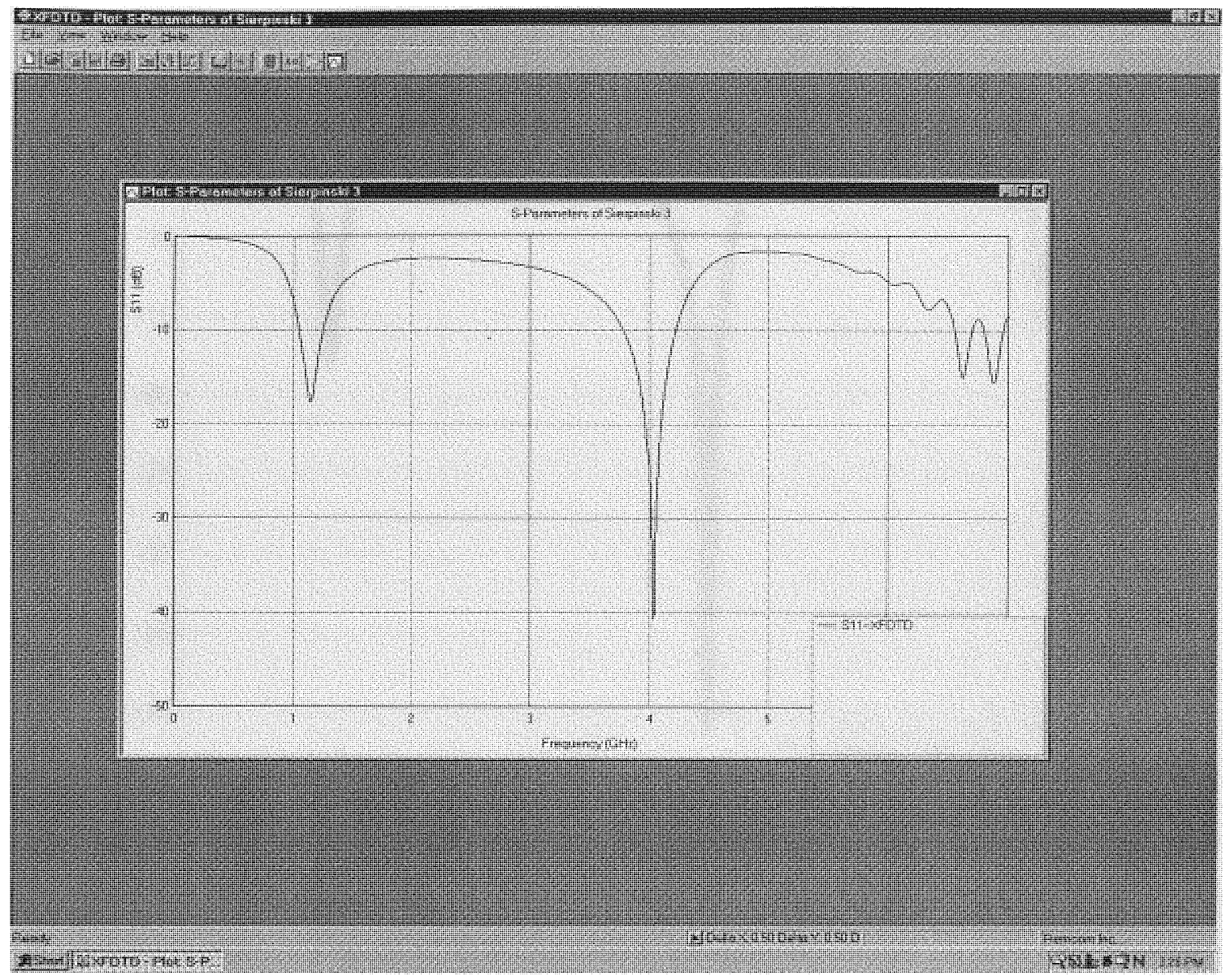

Figure $97 S_{11}$ parameter of Sierpinski 3 


\subsection{Return Loss Sierpinski 3}

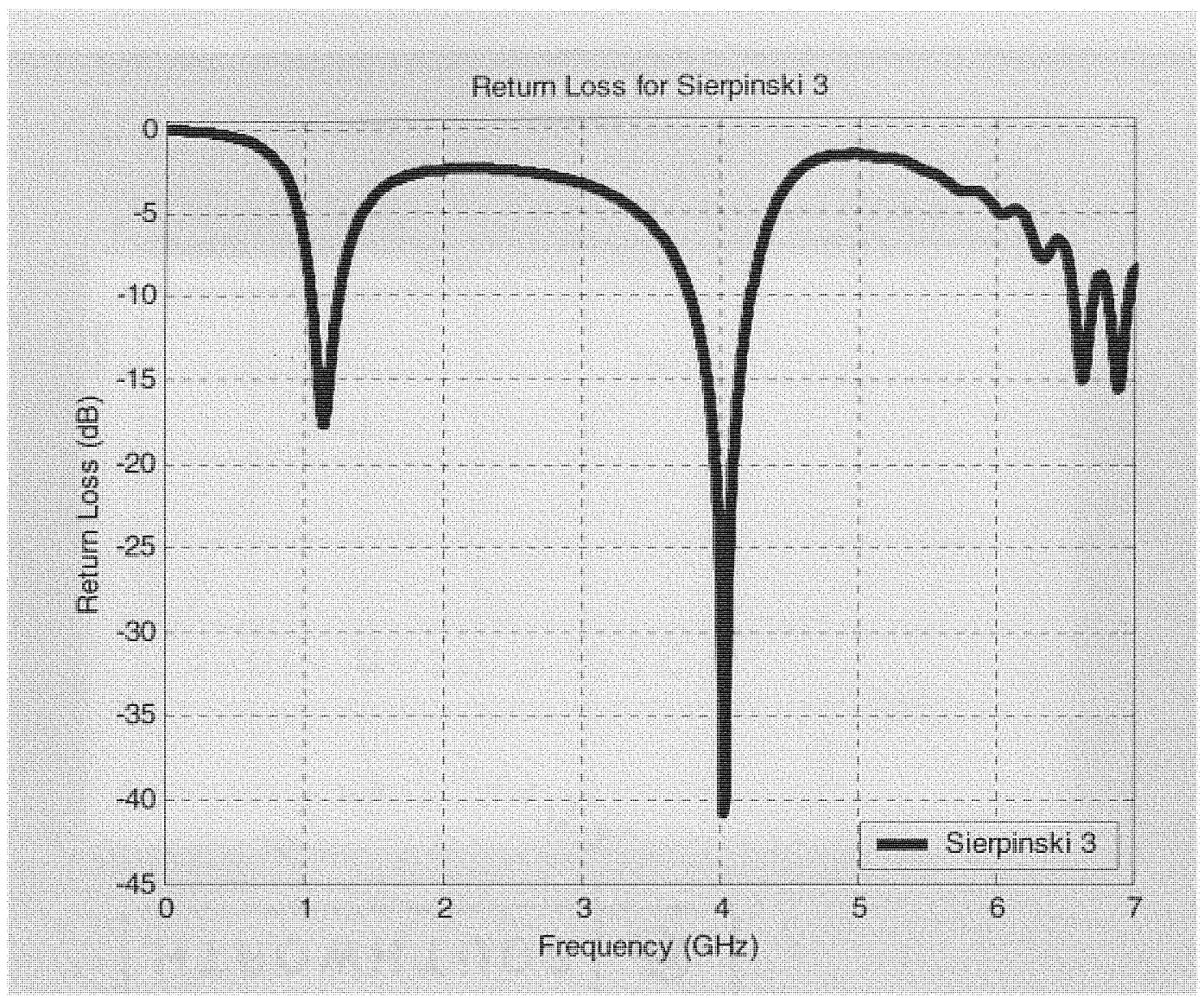

Figure 98 Return Loss of Sierpinski 3 Fractal

The figure 98 above shows the return loss for the sierpinski 3 fractal. This was used to validate the results obtained from the FDTD simulation. The impedance bandwidth obtained from the numerical calculations of the Sierpinski 3 fractal has an operational bandwidth ranges from $1.23 \mathrm{GHz}, 4.2 \mathrm{GHz}, 6.5 \mathrm{GHz}$ and $6.9 \mathrm{GHz}$. It was found that the S11 response for the Sierpinski 3 at the $1^{\text {St }}$ resonant frequency of $1.23 \mathrm{GHz}$ was $-18 \mathrm{~dB}$, the $2^{\text {nd }}$ resonant frequency at $4.2 \mathrm{GHz}$ was $-42 \mathrm{~dB}$, the $3^{\text {rd }}$ frequency of 6.5 $\mathrm{GHz}$ is $-15 \mathrm{~dB}$ and the $4^{\text {th }}$ frequency of $-16 \mathrm{~dB}$ was $6.9 \mathrm{GHz}$. 


\subsection{XFDTD Gain plot of Sierpinski 3}

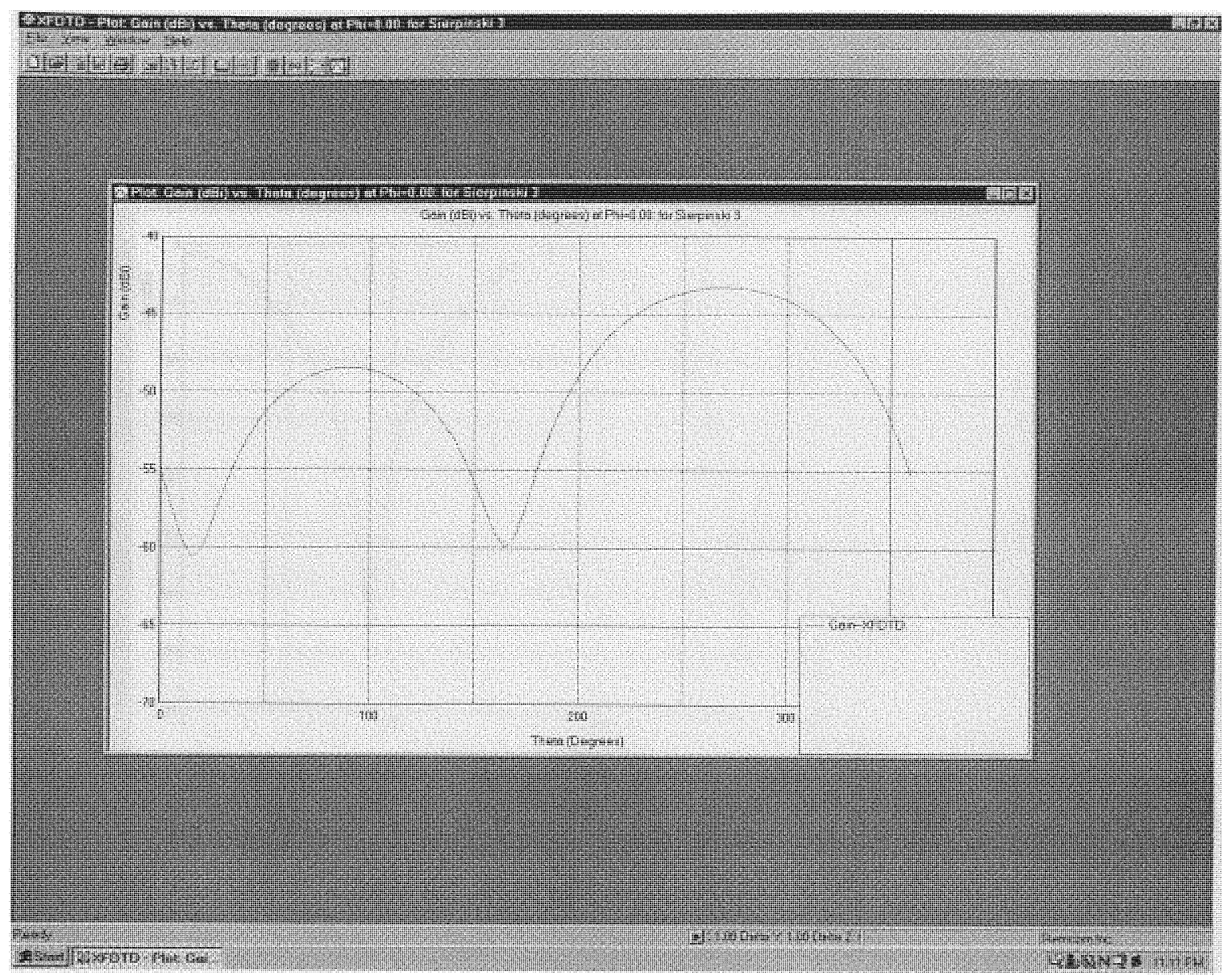

Figure 99 FDTD Simulated Gain of Sierpinski 3

The above figure shows the Gain vs Angle XFDTD simulation. 


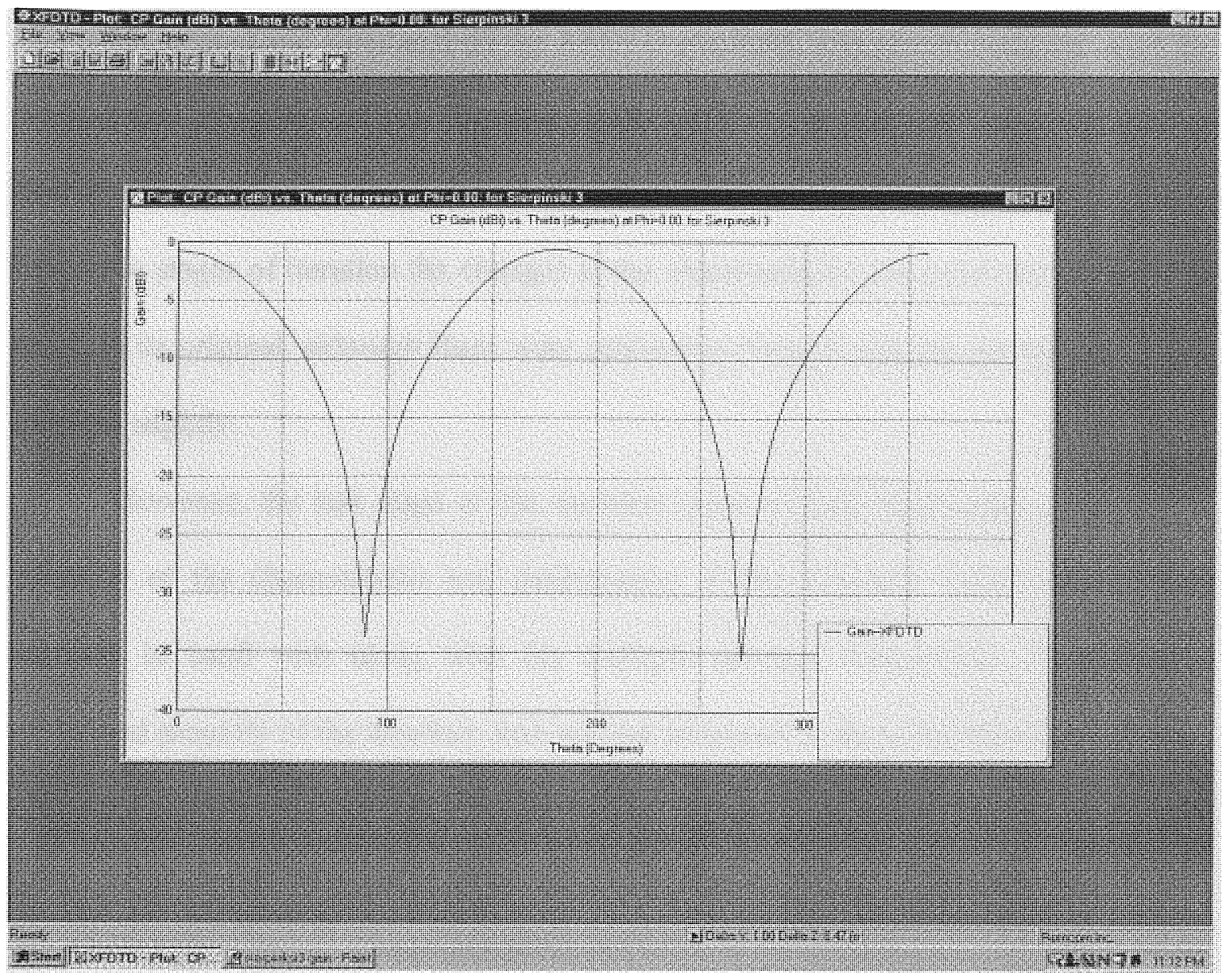

Figure 100 FDTD Simulated CP Gain of Seirpinski 3

The above figure shows the Gain vs Angle XFDTD simulation at Constant Phi. 


\subsection{Results / Discussion}

The Sierpinski gasket structure was presented in this thesis report. The structure was implemented and simulated on the XFDTD platform. The results were analyzed and compared to a bowtie antenna. From the results taken, it was seen that this fractal antenna has an improved bandwidth as the number of iteration increases. It was seen that for the first three stages of iteration the changes in the characteristics and performance were not much to its original, although there were noticeable changes and the resonant frequency changes slightly.

However, the last stage of the iteration show a much significant change in the behavior of the characteristic and performance of the structure. Noticeable, was the increase in band with along with the multiple resonant frequencies. This shows a multiband property of the structure that was not presented in the initial bowtie structure. 
The figure below shows the Return Loss of Sierpinski Antenna with various fractal iterations.

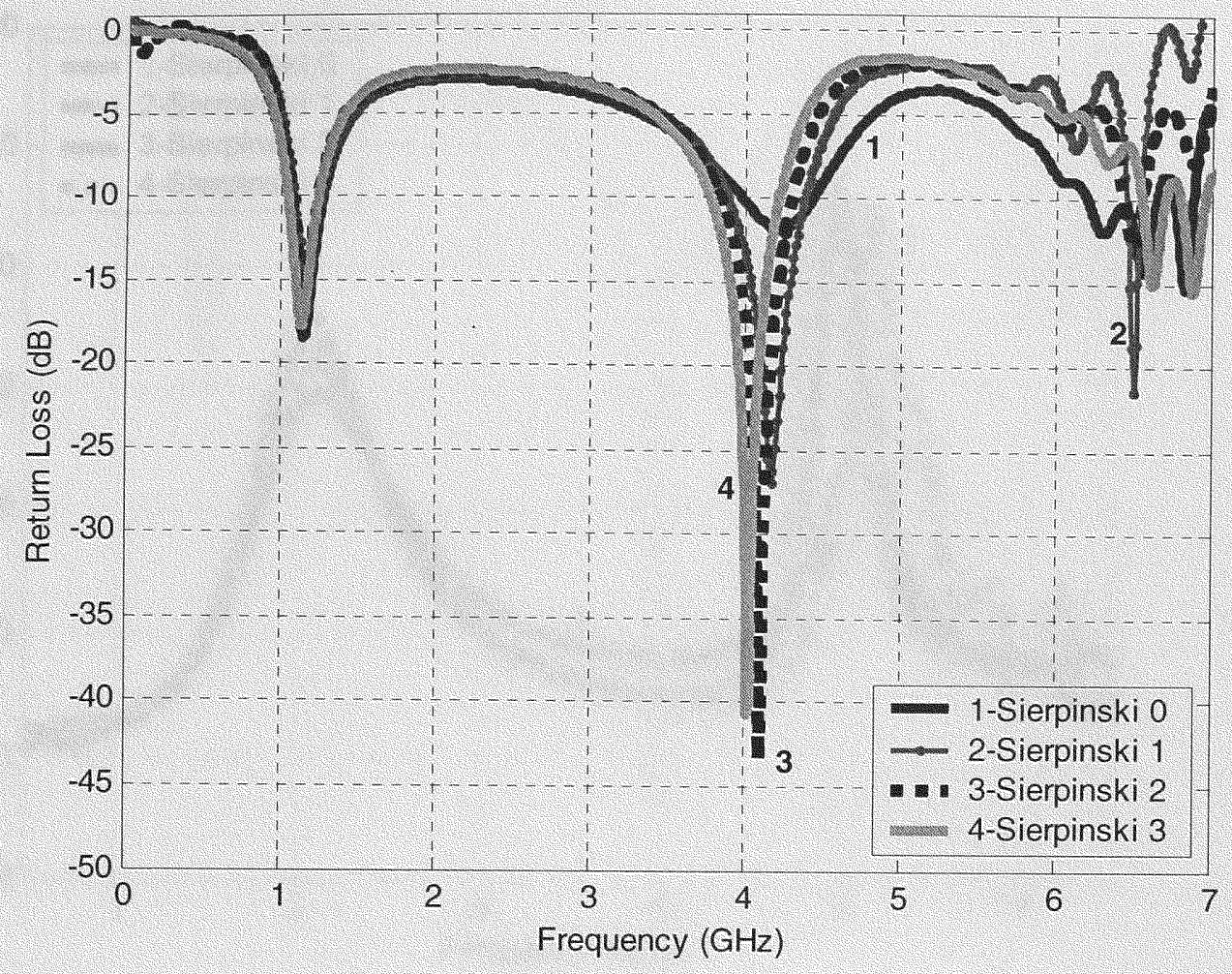

Figure 101 Return Loss of Sierpinski Antenna with various fractal iterations

The XFDTD simulation demonstrated that with increasing iteration, the Sierpinski gasket have multiband properties, figure 101 . One very interesting observation is the increase in bandwidth of the Sierpinski gasket geometry in which it presents about a $20 \%$ bandwidth increase. It is seen that Sierpinski 1,2 and 3 fractals exhibits an almost steady part of the S11 response below $-10 \mathrm{~dB}$. It could be seen that sierpinski 1, 2 and 3 exhibit a better return loss of $-27 \mathrm{~dB},-44 \mathrm{~dB}$ and $-42 \mathrm{~dB}$ respectively. 
Figure 102 below shows the variation in input impedance for the Sierpinski gasket fractal antenna with different iteration stages

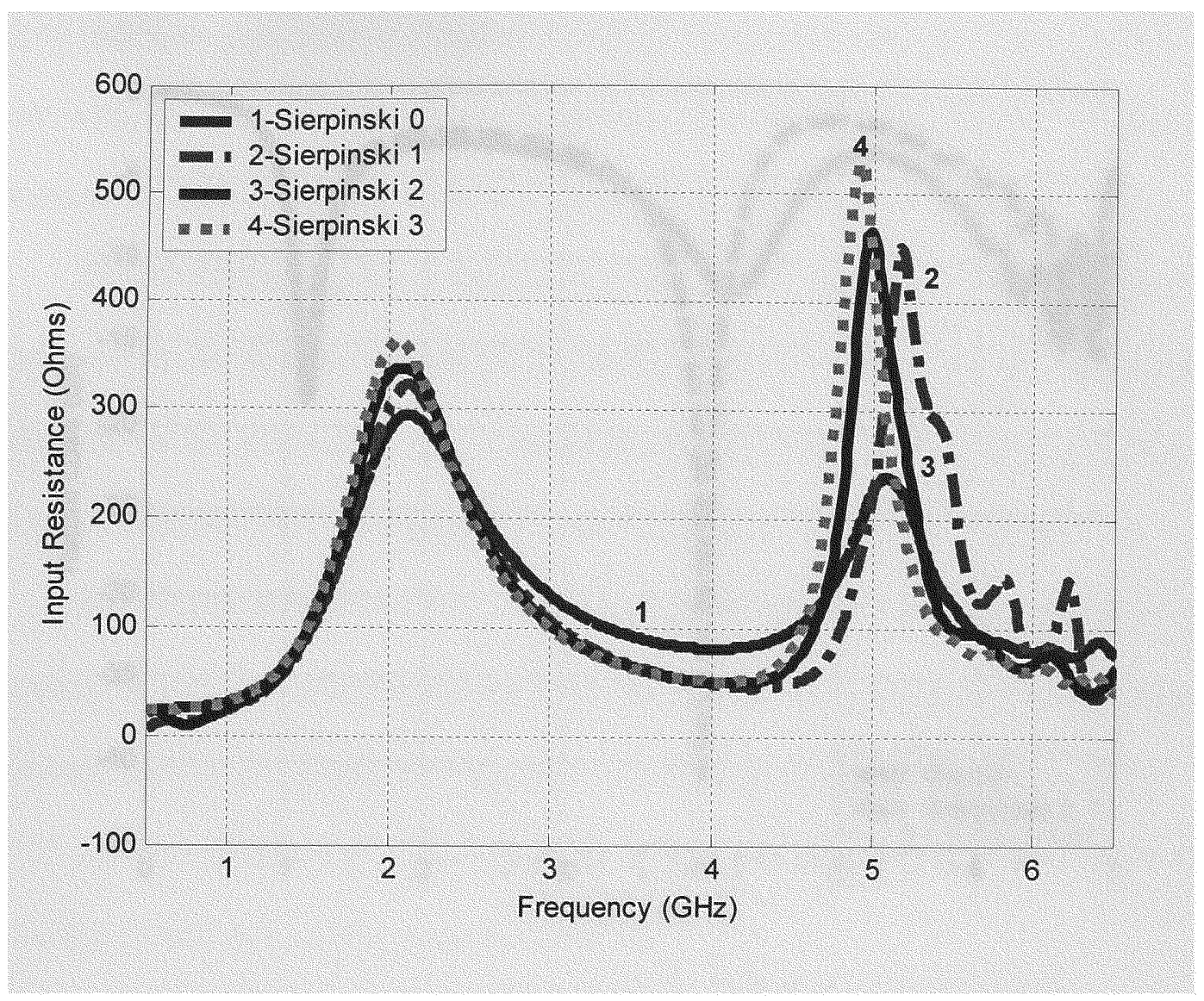

Figure 102 Input Resistance for Sierpinski Fractal at various iterations.

It can be clearly seen that the input impedance of the Sierpinski fractal varied according to different iterations. This is a very useful characteristic because by adjusting the iteration stages we were able to control a very important property of the antenna. 
Figure 103, below shows the return losses of the Bowtie and the final iteration stage of Sierpinski 3 fractal.

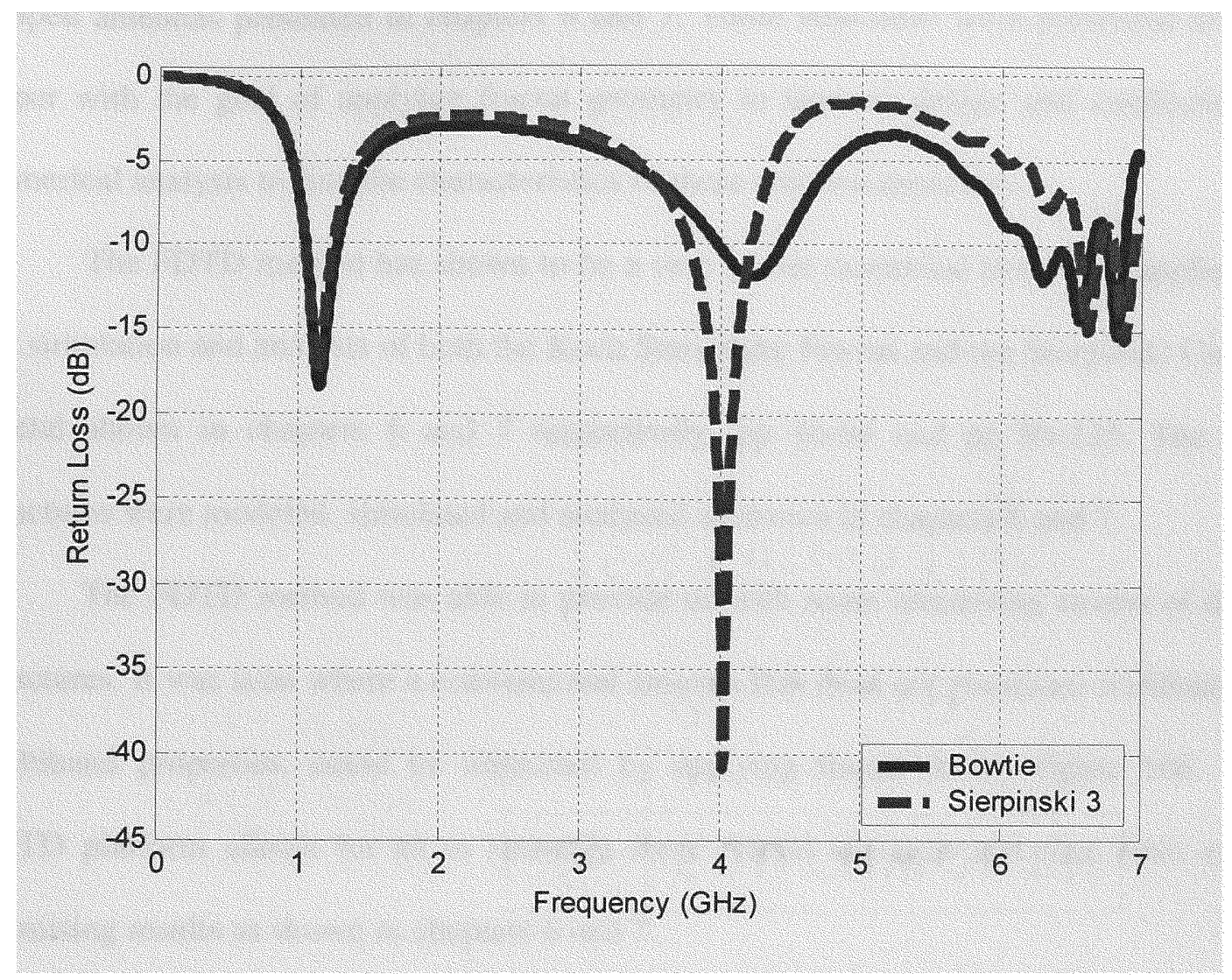

Figure 103 Comparison of Return Loss for Bowtie and Sierpinski 3

The figure above shows the comparison of a bowtie and a $4^{\text {th }}$ order sierpinski fractal antenna. The result shows that the sierpinski antenna has a better return loss. It can be seen that the sierpinski has a better return loss of $-42 \mathrm{~dB}$ compare to the bowtie which has a return loss of $-19 \mathrm{~dB}$ 


\section{Chapter 8 Conclusions}

In this thesis work the FDTD numerical method was used to analyze two fractal shaped antennas presented in chapters 6 and 7 . These structures were presented in this paper with the goal of applying fractal geometry to antenna design and conducting a numerical analysis to find the characteristics of these antenna structures.

The FDTD method has shown to be a very power numerical tool when applied to the simulation and analysis of both the Koch Snowflake Fractal and the Sierpinski Gasket fractal shown in chapters 6 and 7 respectively, pp 46-94 and pp 95-135. The two structures were modeled, simulated and analyzed as shown in chapters 6 and 7 .

The FDTD method was able to provide us with some interesting results of these structures. It was seen where a conventional antenna that does not possesses wideband or multiband properties, could be improved by applying fractal theory figure 106 . The FDTD platform allows for us to structure these fractal antennas and thus have some promising results as shown in chapters 6 and 7 . 
The figure below shows the comparison of the return losses of the three antenna structures that were studied.

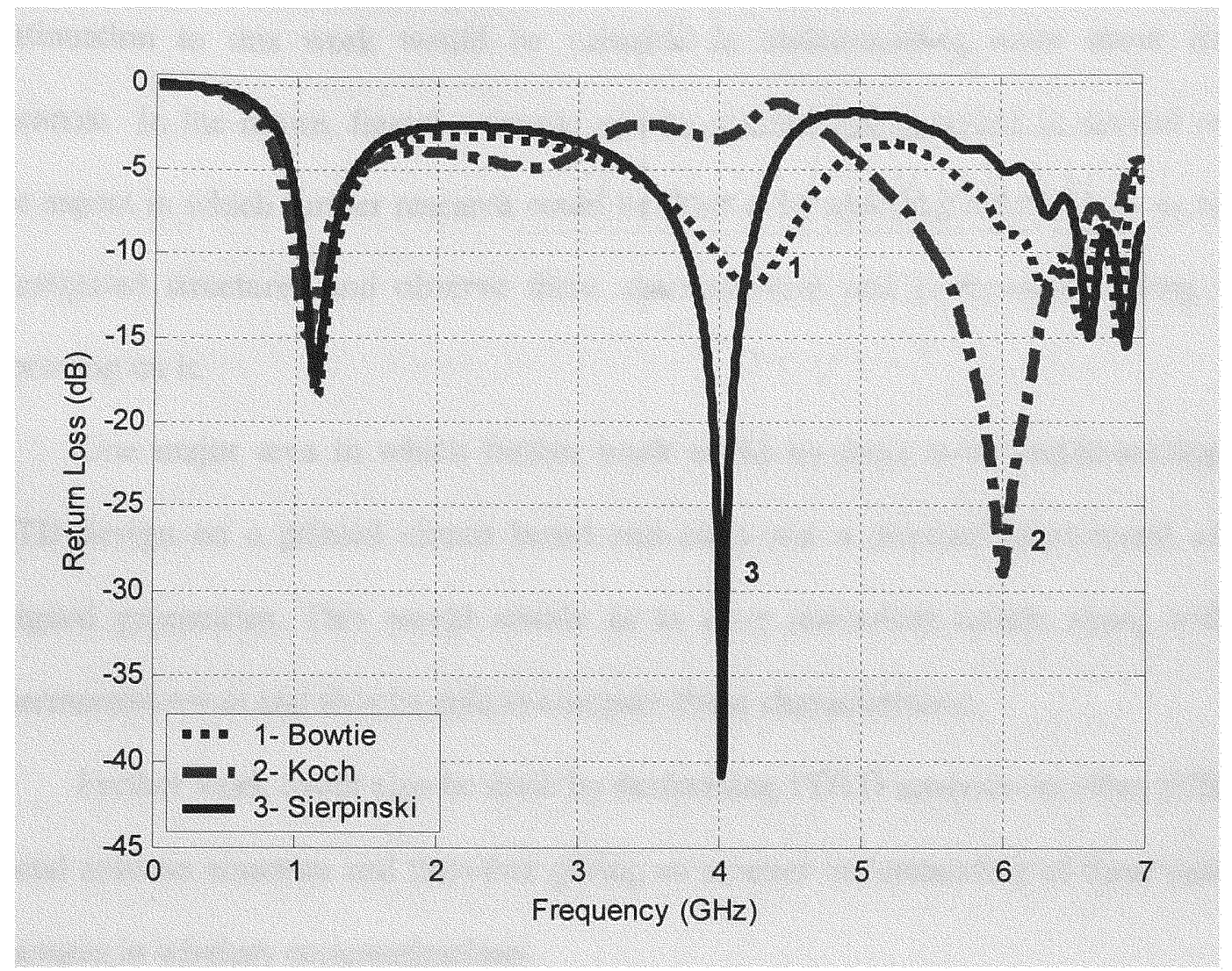

Figure 104 Return Loss of Bowtie, Sierpinski and Koch fractal

In addition the FDTD method also shows fractal shaped antennas provides us with a better performing antenna than its counterpart as shown in figure 104. For this reason, the use of this method is very appropriate in the demands of the required analysis. 


\section{Chapter 9 Future Work}

With the study and investigation that was presented in this thesis a reasonable continuation to this work would be valuable in understanding more about fractal antennas. In the future, fractal antennas can be studied and analyzed in several areas. One aspect in which further research could be done is by applying more iterations to the investigated structures and observe there characteristics and performance along with improving on it.

One major area in which further work could be done is by implementing the FDTD design on a printed circuit board and carry out a physical experiment of the designed geometries. This would enable us to have numerical results along with an experimental result and thus be able to compare these characteristics.

Further work could also be done by performing FDTD analysis on other different Fractal antenna structure and therefore giving us a better understanding of these antenna structures in wireless communications. 


\section{Reference:}

1. Constantine A. Balanis "Antenna Theory Analysis And Design", Second Edition, John Wiley and Sons, Inc,1997

2. K.Falconer, "Fractal Geometry", Mathematical Foundations and Applications, Wiley, NewYork, 1990

3. K.Kunz and R.J Lubbers, "The Finite Difference Time Domain Method for Electromagnetics." CRC Press Inc. 1993.

4. Recom Inc, "User's Manual for XFDTD," Version 5.0.4.9, September 1999.

5. K. S. Yee, Numerical Solution of Initial Boundary Value Problems Involving Maxwell's Equation in Isotropic Media," IEEE Transaction on Antennas and Propagation, Vol. AP-14, May 1966, pp.302-307.

6. G.Mur, "Absorbing Boundary Condition for the Finite Difference Approximation of the Time Domain Electromagnetic Field Equation", IEEE Transactions on Electromagnetic Compatability, Vol. EMC-23, November 1981, pp.377-382.

7. A.Taflove, and M.E Brodwin, "Numerical Solution of Steady-State Electromagnetic Scattering Problems Using the Time-Dependent Maxwell's Equations", IEEE Trans. Microwave Theory Tech, MTT .23, 1975, pp.623-630.

8. B.B. Mandelbrot, "The Fractal Geometry of Nature", W.H. Freeman and Company, 1983.

9. H.O. Peitgen, H.Jürgens, D.Saupe, "Chaos and Fractals", New Frontiers of Science, Springer-Verlag, 1990.

10. M.F. Barnsley , R.L. Devaney, B.B. Mandelbrot, H.O. Peitgen, D. Saupe, R.F. Voss, Y. Fisher, M. Mc Guire, "The Science of Fractal Images", Springer Verlag, 1988.

11. N.Cohen, "Fractal Antennas: Part 1", Communications Quarterly, Summer 1995, pp. 7-22.

12. N.Cohen, "Fractal and Shaped Dipoles", Communications Quarterly, Spring 1996, pp. 25-36. 
13. N.Cohen, "Fractal Antennas: Part 2", Communications Quarterly, Summer 1996, pp.53-66.

14. N.Cohen, R.G.Hohlfeld, "Fractal Loops And The Small Loop Approximation", Communications Quarterly, Winter 1996, pp.77-81.

15. D.H.Werner, P.L.Werner, "Frequency-independent features of self-similar fractal antennas", Radio Science, Volume 31, No.6, November-December 1996, pp.1331-1343.

16. C. Borja and J. Romeu, "On the Behaviour of Koch Island Fractal Boundary Microstrip Patch Antenna," IEEE Trans. Antennas and Propagation., vol. 51, No. 6, Jun. 2003, pp $1281-1291$.

17. C.Puente, J.Romeu, R.Pous, J.Ramis, A.Hijazo, "Small but long Koch fractal Monopole", IEE Electronics Letters, vol.34, no.1, January, 1998, pp.9-10.

18. J.S. McLean, "A Re-Examination of the Fundamental Limits on the Radiation Q of Electrically Small Antennas", IEEE Trans. on Antennas and Propagation, Vol.44, no.5, May 1996, pp.672-675

19. C. Puente, J. Romeu, R. Pous, and A. Cardama, "On the Behaviour of the Sierpinski multiband antenna," IEEE Trans. Antennas and Propagation., vol. 46, Apr. 1998, pp. 517-524.

20. C.Puente, "Fractal Antennas", Ph.D. Dissertation at the Dept. of Signal Theory and Communications, Universitat Politècnica de Catalunya, June 1997.

21. C.Puente, J.Romeu, R.Pous, A.Cardama, "Multiband Fractal Antennas and Arrays", in Fractals in Engineering, J.L.Véhel, E.Lutton, C.Tricot editors, Springer, New York 1997.

22. C.Puente,J.Romeu, R.Bartolomé, R.Pous, "Perturbation of the Sierpinski antenna to allocate the operating bands", IEE Electronics Letters, vol. 32, no.24, November 1996, pp. 2186-2188.

23. C.Puente,J.Romeu,R.Pous,X.Garcia, F.Benítez, "Fractal Multiband Antenna Based on the Sierpinski Gasket", IEE Electronics Letters, vol.32, no.1, January 1996, pp.1-2. 
24. C.Puente, R. Pous, "Fractal Design of Multiband and Low Side-Lobe Arrays", IEEE Transactions on Antennas and Propagation, vol.44, no.5, May 1996, pp.730-739. 